\title{
Nanostructure dictating thermal transport in heterogeneous material systems
}

\author{
A Dissertation Presented to the \\ Faculty of the School of Engineering and Applied Science \\ University of Virginia \\ In Partial Fulfillment \\ of the Requirements for the Degree of \\ Doctor of Philosophy \\ Mechanical and Aerospace Engineering
}

Matthew Linton Bauer

December 2014 


\section{APPROVAL SHEET}

The dissertation is submitted in partial fulfillment of the requirements for the degree of

Doctor of Philosophy in Mechanical and Aerospace Engineering

\section{Matthew L. Bauer}

Author

This dissertation has been read and approved by the Examining Committee:

\section{Pamela M. Norris}

Advisor

Patrick E. Hopkins

Chairman

Gavin T. Garner

\section{Hossein Haj-Hariri}

\section{Arthur W. Litchenberger}

Accepted for the School of Engineering and Applied Science:

\section{James H. Aylor}

Dean, School of Engineering and Applied Science 


\begin{abstract}
Advances in microfabrication technology have led to novel devices requiring the support of microscale material systems with optimized mechanical and thermal properties. Control of thermal energy is necessary for the successful performance of such microdevices. Heterogenous material systems with nanoscale structural features present an opportunity to tune the unique combinations of properties sought for microscale devices outside the limit of traditional, homogenous material systems. The structure can dictate the paths of individual thermal energy carriers which ultimately influence the operating temperature and rate of heat removal from the device. However, the complexity involved in understanding thermal transport in a material system comprised of multiple materials makes predicting the effective thermal conductivity challenging.

This study examines how systematically changing fabrication processes can tune structural attributes in heterogenous systems and thereby tune thermal transport within the system. The $3 \omega$ measurement technique is used to determine the effective thermophysical properties of the systems of interest. The $3 \omega$ technique utilizes a thin metal line as both the heat source and thermometer. An alternating current passes through the line at varying frequency. The third harmonic voltage drop is measured via a lock-in amplifier. The experimental technique is advanced to isolate thermal properties in scenarios where the more traditional experimental usage would not be possible by using a heating wire not directly fabricated onto the sample. This allows for measurement of systems on which a metal line could not otherwise be deposited nor maintained while a controlled loading pressure is applied to the material system.

Some microdevices such as chemical sensors have shown peak efficiency at elevated operating temperatures. Homogenous material systems rarely exhibit
\end{abstract}


thermal conductivities much below $1 \mathrm{~W} / \mathrm{m} \mathrm{K}$. In order to minimize the power required to maintain such operating temperatures, a superior form of insulation with microscale dimensions is sought. Monolithic aerogels have been shown to be capable of achieving thermal conductivities below $0.01 \mathrm{~W} / \mathrm{m} \mathrm{K}$. Their unique microstructure is critical to achieving such a low thermal conductivity. New fabrication processes have shown thin film aerogels capable of achieving much of the unique structure obtained by their monolithic counterparts. In this study, microfabrication processes are developed to determine the thermal transport properties of thin film silica aerogels as well as their direct applicability as thermal insulation in microscale applications. These methods are built upon by systematically tuning the fabrication conditions of the aerogel films. Changes in structure caused by the varied fabrication conditions are measured with grazing-incidence small angle x-ray scattering. The effects of fabrication and the microstructure of the aerogel films on the thermal conductivity and heat capacity of the system are considered.

Carbon allotropes have shown a tremendous range of achievable thermal transport properties as well as mechanical properties. Considering only individual carbon fibers, thermal conductivities spanning four orders of magnitude have been measured. Modeling thermal transport is complicated in carbon fiber composite systems in which the intrinsic properties of individual fibers, their collective density and orientation, the properties of the host material (air in this work), and the interactions between the fibers as well as between the fiber and the host all influence the effective thermal conductivity of the system. The system can be further altered by changing the pressure of the atmosphere, the applied load of the system, or by heat treatment of the system. To give confidence to predictive thermal modeling of such a system, a bidirectional modification of $3 \omega$ was employed to systematically determine the thermal transport of such a system. Fabrication and testing conditions are systematically altered to isolate the roles through which specific 
attributes of the composite contribute to thermal transport. Furthermore, Raman spectroscopy and small angle x-ray scattering are employed to isolate properties contributing to the thermal conductivity of the individual fibers. Predictive thermal modeling has been employed to explain measured results and better illuminate how further tuning of the system could be used to achieve desired thermal properties.

Individual carbon nanotubes have been shown to have thermal conductivities well over 1,000 W/m K. Such high thermal transport properties are desired for removing heat generated by computer processors. However, attempts to obtain ultra-high thermal conductivities in composite carbon nanotube systems have not yielded the expected success. Vertically aligned carbon nanotube arrays (VACNT), which could be used in microscale heat removal applications, are most commonly grown using chemical vapor deposition (CVD) techniques. To gain insight into the effective thermal conductivity of VACNTs, arrays are grown under systematically varied growth conditions using CVD methods and the thermal conductivity measured with the bidirectional $3 \omega$ modification. Raman spectroscopy and transmission electron miscroscopy are then employed to gain insight into the microstucure and ultimately relate its properties to the measured thermal conductivity.

The individual study of thin film silica aerogels, turbostratic carbon fiber networks, and vertically aligned carbon nanotube arrays aims to provide insight into fabrication mechanisms that can be used to alter thermal transport. Relating the varying microstructure of the systems to measured thermal conductivity values enhances the understanding of which mechanisms are most heavily influencing thermal transport. Conversely, the role of specific structural features in tuning thermal transport can be better understood, improving the ability to engineer novel microscale technologies to control thermal transport within a device. 
Dedicated to my wife, Casey:

without you, I wouldn't be here today. 


\section{Acknowledgements}

I am constantly amazed by how little I know. Over the course of my time as a doctoral candidate I have learned that any lack of knowledge can be overcome with time and intellectual euriosity ferocity. However, if I was left in a vacuum unable to communicate with others, I may have spent an entire lifetime unable to reach any conclusions of scientific merit worth writing in this document. I am grateful for all those who shared time and energy with me so that I might become slightly less ignorant on a range of topics. To this day, I am not sure how I snuck into a laboratory comprised of such smart individuals, and I sincerely thank my advisor, Dr. Norris, for providing me this opportunity, mentorship, and financial support.

Thank you Dr. Gavin Garner, Dr. Hossein Haj-Hariri, Dr. Arthur Litchenberger, and Dr. Hopkins for taking the time to serve on my doctoral committee. Dr. Garner helped keep me between the rails during my earliest attempts at assembling $3 \omega$. I must also thank Dr. Donald Jordan for patiently directing me, a frustrating first year graduate student, during this same time. Dr. Litchenberger guided my efforts in microfabrication and through many conversations saved me countless hours of failed fabrication. Dr. Hopkins has been both a mentor and friend, from our brief months of overlap as he graduated from Dr. Norris' lab, to his perpetual open door to talk about anything on my mind, scientific or otherwise.

The stories of students' tenure at graduate school are often heavily shaped by their lab-mates. My story could not be told without highlighting the the wealth of guidance from others struggling through similar challenges: Chris Baker, Casey Bauer, John Duda, Timothy English, Christina Haden, Michael Fish, Nam Le, Roy Mathews, Christina Pappas, MacKenzie Redding, Rich Salaway, Chris Saltonstall, and Justin

Smoyer. I must particularly thank Roy for hundreds of hours dedicated to making me proficient at microfabrication, Mike for the blood, sweat, and tears put into making $3 \omega$ operational, Chris for being my Raman guy, and Casey for working long hours 
to fabricate then teach me how to fabricate thin film aerogels. Additionally, Quang Pham has been perhaps the best undergraduate researcher I could have wished to work with and has been instrumental in my studies of carbon nanotubes.

Finally, my family specially warrants thanks for any success I've had to this point in my life. Bruce and Denise Bauer, my parents, have given me the great gift of opportunity. Every door I could have wished to travel through was open and available to me. Casey, my fiancé when I began graduate school, has always pushed me to be something better and has been there to catch me when I have slipped. She has been my greatest collaborator (that is no exaggeration) and the friend I needed when life was been more than I could handle. 


\section{Nomenclature}

\section{Greek Symbols}

$\alpha \quad$ Fluid thermal diffusivity $\left[\mathrm{m}^{2} \mathrm{~s}^{-1}\right]$

$\alpha \quad$ Term accounting for contact resistance

$\beta \quad$ Coefficient of thermal expansion

$\delta \quad$ Perturbation parameter

$\lambda \quad$ Mean free path $[\mathrm{nm}]$

$\lambda \quad$ Wavelength $[\mathrm{m}]$

$\mu \quad$ Poisson's ratio

$\omega \quad$ Angular frequency $[\mathrm{Hz}]$

$\phi \quad$ Material percent composition

$\pi \quad$ Mathematical consent, ratio of a circle's circumference to its diameter

$\rho \quad$ Density $\left[\mathrm{kg} \mathrm{m}^{-3}\right]$

$\sigma \quad$ Electrical conductivity $\left[\mathrm{S} \mathrm{K}^{-1}\right]$

$\tau \quad$ Scattering rate $\left[\mathrm{s}^{-1}\right]$

$\theta \quad$ Exit angle 


\section{Roman Symbols}

A Temperature matrix above the heater

$B \quad$ Temperature matrix below the heater

$b \quad$ Heater width $[\mathrm{m}]$

$C \quad$ Volumetric heat capacity $\left[\mathrm{J} \mathrm{m}^{-3} \mathrm{~K}^{-1}\right]$

c Specific heat capacity $\left.\left[\mathrm{J} \mathrm{kg}^{-1} \mathrm{~K}^{-1}\right]\right]$

$D \quad$ Thermal diffusivity $\left[\mathrm{m}^{2} \mathrm{~s}^{-1}\right]$

$d \quad$ Diameter $[\mathrm{m}]$

$d \quad$ Material layer thickness [m]

$D_{m} \quad$ Mass fractal dimension

$D_{s} \quad$ Surface fractal dimension

E $\quad$ Energy $[\mathrm{J}]$

E Young's modulus

$f \quad$ Frequency $[\mathrm{Hz}]$

$g \quad$ Acceleration due to gravity $\left[\mathrm{m} \mathrm{s}^{-2}\right]$

I Current $[\mathrm{A}]$

I Intensity

$i \quad$ Imaginary unit

$k \quad$ Thermal conductivity $\left[\mathrm{W} \mathrm{m}^{-1} \mathrm{~K}^{-1}\right]$

Kn Knudsen number 
$L \quad$ Material's characteristic length [nm]

$l \quad$ Heater length $[\mathrm{m}]$

$L_{a} \quad$ Coherence length $[\mathrm{m}]$

$m \quad$ Integration variable

m Slope

n Porod exponent

$P \quad$ Input power [W]

P Porosity

$p \quad$ Applied pressure

$P_{1} \quad$ Individual contact load

$q \quad$ Heat flux $\left[\mathrm{W} \mathrm{m}^{-2}\right]$

$q \quad$ Scattering vector $\left[\mathrm{m}^{-1}\right]$

$R \quad$ Electrical resistance $[\Omega]$

$R \quad$ Interfacial resistance $\left[\mathrm{W} \mathrm{m} \mathrm{m}^{-2} \mathrm{~K}^{-1}\right]$

$r \quad$ Radius $[\mathrm{m}]$

$r_{g} \quad$ Radius of gyration $[\mathrm{m}]$

Ra Rayleigh number

$S \quad$ Material structure matrix

$S \quad$ Normalized sensitivity

$S \quad$ Seebeck coefficient $\left[\mathrm{V} \mathrm{K}^{-1}\right]$ 
$T$ Temperature $\left[\mathrm{K}\right.$ or $\left.{ }^{\circ} \mathrm{C}\right]$

$u \quad$ Thermal wave vector $\left[\mathrm{m}^{-1}\right]$

V Voltage [V]

Z Average orientation

$z \quad$ Position relative to heating wire

ZT Thermoelectric figure of merit

\section{Superscripts}

$+\quad$ Thermal wave positive propagation

- $\quad$ Thermal wave negative propagation

\section{Subscripts}

$0 \quad$ Unperturbed response

$1 \omega \quad$ First harmonic signal or response

$2 \omega \quad$ Second harmonic signal or response

$3 \omega \quad$ Third harmonic signal or response

$B \quad$ Property in a bulk or infinite medium

E Effective value when all constituent materials are accounted for

f $\quad$ Fiber phase

$g \quad$ Gas phase

$h \quad$ Heating layer

$h \quad$ Host material 
$i \quad$ General material interface

j General material layer

$p \quad$ Included material

$p \quad$ Parameter of interest

$r \quad$ Property ratio

$Z \quad$ Property at a given orientation 


\section{Contents}

Nomenclature $\quad$ iv

1 Introduction 1

1.1 Background ........................... 1

1.2 Thermoelectric devices ..................... 4

1.3 Microscale sensors . . . . . . . . . . . . . . . . . . . 6

1.4 Thermal interface materials . . . . . . . . . . . . . . 8

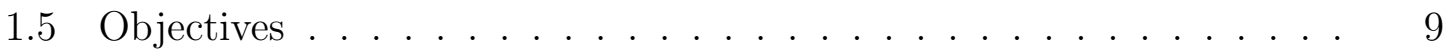

2 Thermal Transport in Heterogenous Material Systems 11

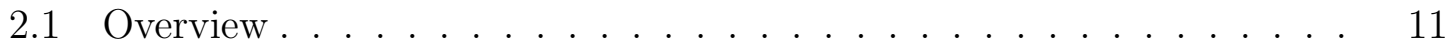

2.2 Single phase solid . . . . . . . . . . . . . . . . . . . 11

2.3 Single phase gas . . . . . . . . . . . . . . . . . . . . . . 15

2.4 Heterogenous material systems . . . . . . . . . . . . . . . . 17

2.5 Thermal resistance at an interface . . . . . . . . . . . . . . 19

2.6 Summary ............................. 20

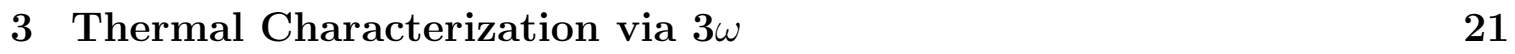

3.1 Review of third harmonic experiments . . . . . . . . . . . . . 21

$3.23 \omega$ system implementation and measurement considerations . . . . . . 23

3.2.1 Determining a sample's thermophysical properties from $V_{3 \omega}$. . 29 
3.2 .2 General sensitivity . . . . . . . . . . . . . . . . 33

3.2 .3 Initial $\mathrm{SiO}_{2}$ test . . . . . . . . . . . . . . 36

3.3 Development of a bidirectional $3 \omega$ system . . . . . . . . . . . 37

3.3 .1 Motivation . . . . . . . . . . . . . . . . . 37

3.3.2 Mounting scheme fabrication . . . . . . . . . . . . . 38

3.3 .3 Experiment operation . . . . . . . . . . . . . . . . 42

3.3.4 Determining thermophysical properties . . . . . . . . . . 44

3.3.5 Parameterized sensitivity analysis . . . . . . . . . . 45

3.3 .6 Sample measurement . . . . . . . . . . . . . . . . . 48

3.3 .7 Sources of error . . . . . . . . . . . . . . . 50

3.3 .8 Summary . . . . . . . . . . . . . . . . . . . 51

4 Thermal Isolation via Thin-Film Silica Aerogels 53

4.1 Background and introduction . . . . . . . . . . . 53

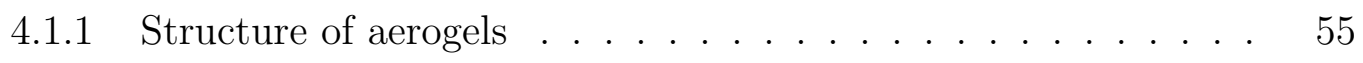

4.1 .2 Aerogel fabrication . . . . . . . . . . . . . 56

4.1.3 Thermal transport studies of aerogels . . . . . . . . . . 60

4.2 Aerogel insulated MEMS . . . . . . . . . . . . . . . . . . 61

4.2 .1 Sol-gel procedures . . . . . . . . . . . . . . . . 62

4.2 .2 Thin-film deposition processes . . . . . . . . . . . . 63

4.2.3 Microfabrication on the samples . . . . . . . . . . . 64

4.2.4 Implementation of $3 \omega$ and sample thermal conductivity measurements . . . . . . . . . . . . . . 66

4.3 Structural tuning study . . . . . . . . . . . . . . . . 71

4.3 .1 Recipe design . . . . . . . . . . . . . . . . . . 73

4.3.2 Structural characterization: SAXS and XRR . . . . . . 75

4.3 .3 Results and discussion . . . . . . . . . . . . . . 82 
5 Pressure Dependent Thermal Transport in Carbon Nanofiber $\begin{array}{ll}\text { Networks } & 94\end{array}$

5.1 Background . . . . . . . . . . . . . . . . . . 94

5.2 Sample preparation . . . . . . . . . . . . . . . . . . . 97

5.3 Fiber characterization . . . . . . . . . . . . . . . 98

5.4 Thermal characterization and modeling . . . . . . . . . . 107

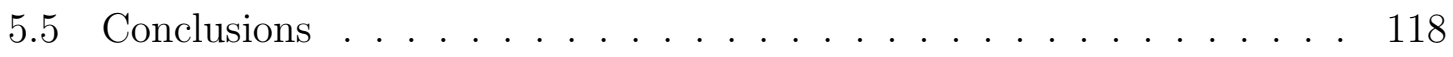

6 Vertically Aligned Carbon Nanotube Arrays for Heat Removal 119

6.1 Background . . . . . . . . . . . . . . . . . . . . . . . . . . 119

6.2 VACNT growth . . . . . . . . . . . . . . . . 121

6.3 Structural characterization . . . . . . . . . . . . . . . . . . 124

6.4 Thermal characterization and discussion f . . . . . . . . 128

6.5 Summary . . . . . . . . . . . . . . . 130

7 Summary and Future Studies 132

7.1 Summary of conclusions . . . . . . . . . . . . . . . . 132

7.2 Suggested future studies $\ldots \ldots \ldots \ldots \ldots$

$\begin{array}{ll}\text { References } & 138\end{array}$ 


\section{List of Figures}

1.1 Scope of optimizing thermal performance. . . . . . . . . . . . . 3

1.2 A thermoelectric generator utilizing the Seebeck effect. . . . . . . . . 4

1.3 Diaphragm for thermally isolating hot plates and sensors at elevated temperatures. ..................... 6

1.4 Silicon nitride diaphragm isolating hot plates by surface machining developed in collaboration with Honeywell. . . . . . . . . . . . . . . . 7

1.5 Schematic of a thermal interface material aiding in removing heat from a circuit element. . . . . . . . . . . . . . . . 8

3.1 Traditional sample characterized via the $3 \omega$ technique. The four contact pads serve as a four point probe connection allowing a voltage reading to be made on the central portion of the thin heating wire without significant interference from the remainder of the electrical circuit. . . . . . . . . . . . . . . . 
3.2 Depiction of a simplified $3 \omega$ circuit. Current passes from the lock-in amplifier through the potentiometer, through the sample heating wire, and is then grounded. Digital multimeters and the lock-in amplifier are connected to each element through a differential amplifier. The multimeters are used to determine the first harmonic voltage drop across each element. These readings are used to determine the sample's electrical resistance (and therefore the amount of heat deposited into the sample). The A and B inputs on the lock-in amplifier read the third harmonic voltage drop across each element. The difference in these values is calculated and used to determine the second harmonic temperature rise in the heating wire and ultimately the sample's thermophysical properties. . . . . . . . 26

3.3 Schematic of $3 \omega$ equipment layout. . . . . . . . . . . . . . . .

3.4 Plot of third harmonic voltage with a normal resistor in place of the sample in the $3 \omega$ circuit. Ideally this data is zero at all points. Deviation from zero is insignificant over most of the frequency range for the in-phase signal. . . . . . . . . . . . . . . . . . . . 28

3.5 Depiction of thermal waves traveling from the heating layer into a three layer system including a semi-infinite substrate. In this configuration the system is generally under vacuum. It is assumed all of the heat leaving the heating wire $(j=4)$ is deposited into the top film. . . . .

3.6 Sensitivity response for a three material system with layers 1, 2 and 3 using the known properties of an amorphous $\mathrm{SiO}_{2}$ substrate, a micron thick silver film and a $200 \mathrm{~nm}$ thick $\mathrm{SiO}_{2}$ film respectively. The input heater width was 30 microns. . . . . . . . . . . . . 
3.7 Sensitivity plot considering thermal boundary resistance for a three layer system. The top and bottom layers are amorphous $\mathrm{SiO}_{2}$. The central layer is a thin film aerogel, similar to those studied in Chapter 4 , with an input thermal conductivity of $0.02 \mathrm{~W} / \mathrm{m} \mathrm{K}$ and thickness of $500 \mathrm{~nm}$. The input thermal boundary resistance is $10^{-7} \mathrm{~m}^{2} \mathrm{k} / \mathrm{W} \ldots$.

3.8 Measured thermal conductivity of the first sample tested at UVA, an amorphous $\mathrm{SiO}_{2}$ substrate, compared with data from previous research.[1] 36

3.9 The left image depicts the $3 \omega$ mount with a sample pressed to it. The lowest layer of the mount is a copper slab which attaches to the cryostat. Fabricated onto it from bottom to top is the SU-8 polymer layer, the gold $3 \omega$ pads and heating line, and an $\mathrm{SiO}_{2}$ passivation layer. The pads are connected to the remainder of the circuit via silver paste. The sample is pressed onto the central portion of the metal line by an external force. The right image represents these six layers (with index $j$ ) with the apparent stratified medium perspective of the system. Interfaces, labeled $i$, separate each layer. Thermal waves propagate from the central heating layer $(j=3)$ in both the $+z$ direction toward passivation layer, sample, and substrate $(j=4,5,6)$, and the $-z$ direction into SU-8 photoresist and copper base $(j=2,1)$.

3.10 Crack in the polymer identified after the deposition and etching of the gold metal line. . . . . . . . . . . . . . . . . . 
3.11 Plots of fitting parameter sensitivity trends over a wide frequency range in the six layer system. In all scenarios the sample of interest is set to a thickness of $90 \mu \mathrm{m}$ and a volumetric heat capacity of $1 \mathrm{MJ} / \mathrm{m}^{3} \mathrm{~K}$ on an amorphous $\mathrm{SiO}_{2}$ substrate at room temperature. The passivation layer, $\mathrm{SiO}_{2}$, has an input thermal conductivity of $0.7 \mathrm{~W} / \mathrm{mK}$ and a thickness of $50 \mathrm{~nm}$ unless otherwise stated. The sample's thermal conductivity is $1 \mathrm{~W} / \mathrm{m} \mathrm{K}$ in subfigures (b), (c), and (d). The contact resistance is set to $3 \times 10^{-5} \mathrm{~m}^{2} \mathrm{~K} / \mathrm{W}$ in subfigures (a), (c), and (d). The copper mount layer is assumed to have bulk properties. . . . . . . . . . .

3.12 In-phase $V_{3 \omega}$ data collected and best fit results of the experiments for three scenarios: the mount under vacuum with no sample, and the sample present at two unique pressures. All data was collected at 297

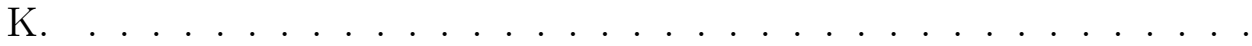

4.1 Different length scales of aerogel features.[2] The mesoporous silica chains contain clumps of silica molecules that make a random walk throughout the material system. The microporous silica clumps are capped by terminal groups. These clumps are not generally neat, geometrically defined groups. Rather, depending on the sol-gel process, they are highly fractal in nature with complex interconnects and pores between them as depicted in Figure 4.3. In thin films, the macroporous regime can be thought of as an irregularity or incomplete portion of the larger aerogel since pores in that regime are often larger than the film thickness. Therefore, for a thin-film silica aerogel to achieve high porosities, macroporous features must be avoided. . . . . . . . . . . . . . . . .

4.2 Steps and properties of a sol-gel process.[3] . . . . . . . . . . . . 57 
4.3 Depiction of silica network formation. Subfigure a) shows relatively linear chains of the backbone material far from the gelation point. Subfigure b) shows these same chains with greater entanglement and cross linking at the gel point. Subfigures c) and d) show a much more clustered network prior to and after reaching the gel point. . . . . . . 58

4.4 Silylation and its consequences: a) Hydroxyl groups within aerogel pores; b) Introduction of modifying agent; c) Modifying agent reacting with hydroxyl groups and replacing the capping terminal bonds; d) Surface tension causes shrinking; e) Resulting pore if no modifying agent is used in which pore interaction causes permanent siloxane bonds to form; f) Resulting pore due to effects of modifying agent with no further interaction allowing pores to spring back to near the original size . . . . . . . . . . . . . . .

4.5 Schematic of the central portion of the sample after micro fabrication. Heat travels from the metal wire through each layer of the sample. . .

$4.63 \omega$ sensitivity plot to an aerogel's thermal conductivity for the described three layer scenario. Sensitivity response is generated for the outer bounds of likely thermal conductivity $(1 \mathrm{~W} / \mathrm{m} \mathrm{K}$ being near that of amorphous $\mathrm{SiO}_{2}$ and $0.01 \mathrm{~W} / \mathrm{m} \mathrm{K}$ is reasonably less than non convective air) as well as three intermediate scenarios. In generating these plots the assumed aerogel density (and therefore volumetric heat capacity) is varied from assumed $\mathrm{SiO}_{2}$ fill fraction of $100 \%$ to $1 \%$ to more reasonably match potential measured samples. Input aerogel thickness is $1 \mu \mathrm{m} \ldots \ldots \ldots \ldots$. . . . . . . . . . . 67

4.7 Experiment sensitivity to the input parameters of the 3 layer system. $\quad 68$ 
4.8 Exemplary raw data converted from in-phase $V_{3 \omega}$ to in-phase $T_{2 \omega}$ for both an amorphous $\mathrm{SiO}_{2}$ substrate, and sample Aerogel B. Fit lines show region in which the slope method can be used to determine sample thermal conductivity. . . . . . . . . . . . . . .

4.9 Left: side view SEM image of an aerogel thin film formed through process C. Right: bulk aerogel formed through process C. These images confirm that aerogel C's porous structure collapsed during deposition.

4.10 Left: side view of Aerogel B-pryolized after deposition of the quartz and niobium layers. Right: high resolution top view of the same sample prior to the deposition of capping layers. Both images show the highly porous structure of the aerogel remained in tact after deposition of the gel and of the two capping layers. . . . . . . . . . . . . . . 71

4.11 Implementation of a TFSA into a micro fabricated circuit. . . . . . 72

4.12 Grazing incidence small angle x-ray scattering (GISAXS) data collection geometry. Incident beam scatters with the sample. A beam blocker is positioned to block the specular reflection of the beam. Diffuse scattering intensity is measured as a function of the exit angle with the sample. Sizes are not to scale. . . . . . . . . . . 76

4.13 Example of GISAXS raw data taken on an aerogel thin film over a 10 hour period. The specularly reflected portion of the beam is blocked, although a small portion still appears on the detector. Outside of this blocked beam is an intensity profile of collected diffusely scattered $\mathrm{x}$ ray signal which contains key information about pore properties. . . . 77 
4.14 GISAXS data taken on a blank $\mathrm{SiO}_{2}$ substrate in an identical scenario to Figure 4.13. The lack of diffusely scattered energy implies the material studied has a homogenous electron density. The signal detected just outside the beam blocker is created by smearing of the incident $\mathrm{x}$-ray beam in the alignment steps. These artifacts can be subtracted in data analysis, or more simply can be avoided by analyzing data at offset angles. . . . . . . . . . . . . . . . . 78

4.15 An intensity profile of GISAXS data from the beam center toward the edge of the detector. . . . . . . . . . . . . . .

4.16 GISAXS data converted to a Guinier plot at low angles to isolate the pores' average radius of gyration. . . . . . . . . . . . . . . . . 80

4.17 GISAXS data at large exit angles trending towards a power law decay indicating the Porod limit. . . . . . . . . . . . . . . .

4.18 Measured specific heat capacity vs porosity of xerogel and aerogel films. Error bar shown is representative for all samples. Sold line shows the specific heat capacity of of amorphous $\mathrm{SiO}_{2}$. . . . . . . . .

4.19 Measured specific heat capacity vs pore diameter of xerogel and aerogel films. . . . . . . . . . . . . . . . . .

4.20 Measured specific heat capacity vs Porod exponent of xerogel and aerogel films. . . . . . . . . . . . . . . . . .

4.21 Measured thermal conductivity vs porosity of aerogel films. Also plotted is the thermal conductivity predicted from differential effective medium theory. . . . . . . . . . . . . . . .

4.22 Measured thermal conductivity deviation from Equation 4.3 plotted against the Porod exponent for the more mass fractal aerogels. . . . . 
5.1 Average Raman spectra of each sample. Each spectrum was first normalized to the $G$ peak intensity and then averaged. a) The averaged spectra of the unannealed samples. b) Averaged spectra of the annealed S1 series. . . . . . . . . . . . . . . 100

5.2 Five representative spectra for the samples annealed at $\operatorname{HTT} 1100^{\circ} \mathrm{C}$, $1300^{\circ} \mathrm{C}$, and $1500^{\circ} \mathrm{C}$ as well as the relevant non-anneald sample showing how annealing changes the uniformity of the microstructure. a) Spectra of the unannealed sample $S 1_{00}$. b) $S 1_{11}$. The $\mathrm{G}$ peak broadens slightly and shoulder at $1150 \mathrm{~cm}^{-1}$ disappears. c) $\mathrm{S}_{13}$. Some non-uniformity of the sample begins to be present. d) $\mathrm{S}_{15}$. Sample exhibits strong nonuniformity and shows signs of localized graphitization as evidenced by the strong and sharp G peak. . . . . . . . . . . . . . . . . 102

5.3 Guinier plot of SAXS intensity decay. The lack of linear region over this $q$ regime indicates an absence of monodisperse voids observed in other carbon fiber samples. . . . . . . . . . . . . . . . . . . 104

5.4 Scanning electron microscope image of a carbon fiber sample. . . . . 105

5.5 Sensitivity of the $3 \omega$ experiment to composite samples' effective thermal conductivity and volumetric heat capacity as well as the contact resistance between the mount and sample. This plot was generated with $k_{e}=0.024 \mathrm{~W} / \mathrm{m} \mathrm{K}, C_{e}=3 \times 10^{5} \mathrm{~J} /\left(\mathrm{m}^{3} \mathrm{~K}\right)$, and $R_{p}=6 \times 10^{-5} \mathrm{~K} \mathrm{~m}^{2} / \mathrm{W}$, similar to the fourth data point in Figure 5.6. The change in direction of the contact resistance sensitivity above $10,000 \mathrm{~Hz}$ only exists due to the presence of the $\mathrm{SiO}_{x}$ layer closer to the heater. At greater applied pressures, the signal's sensitivity to the carbon fibers' thermal conductivity and heat capacity is increased. At low applied pressures the experiment can lose sensitivity to these

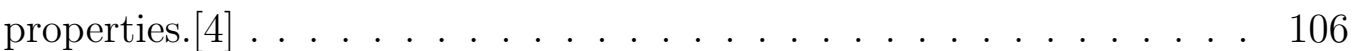


5.6 Best fit results of data taken on $\mathrm{S}_{100}$ with measured density of 90 $\mathrm{kg} / \mathrm{m}^{3}$. Measurements were taken at six unique compressive pressures, with the sample thermal conductivity, sample volumetric heat capacity, and the sample to mount contact resistance simultaneously fit. This data is representative of data taken on each sample. The fitting error propagated from sources of uncertainty in the system is estimated at $9 \%$ for the sample's thermal conductivity, $11 \%$ for the volumetric heat capacity and $6 \%$ for the contact resistance between the sample and the passivation layer. . . . . . . . . . . . . . . . .

5.7 Normalized modeled response of $200 \mathrm{~kg} / \mathrm{m}^{3}$ dense carbon nanofiber composite (S5) as pressure is applied. The total thermal conductivity response, $k_{e}$, only increases due to the role of thermal conductance through fiber contacts. Changing fiber orientation, fill fraction, and air conductivity each act to decrease the composite's thermal conductivity as pressure is applied. . . . . . . . . . . . . . .

5.8 Measured thermal conductivity values from sample S5 at both vacuum and atmospheric pressure. Carbon fill fraction is calculated from the best fit volumetric heat capacity using Equation 5.3. . . . . . . . . 115

5.9 Best fit $3 \omega$ thermal conductivity results for non heat treated samples S1, S2, S3, S4, and S5 (densities of 90, 110, 130, 160 and $200 \mathrm{~kg} / \mathrm{m}^{3}$ respectively). All data is acquired at room temperature and atmospheric pressure. The thermal conductivity predictions from the SFM (dashed lines) are also plotted for initial fill fractions ranging from $4 \%$ to $12 \%$ in steps of $2 \%\left(\phi_{0}\right)$. As pressure is applied the fill fraction increases to a maximum value of $\phi(P)=40 \%$. . . . . . . 116 
5.10 Best fit data from the heat treated sample series (S1). The measured thermal conductivity increases significantly from the as grown sample compared to the heat treated samples, with the sample which underwent a $1500^{\circ} \mathrm{C} \mathrm{HTT}$ showing an additional increase compared to the more moderately heated samples. The change in effective thermal conductivity with respect to carbon fiber fill fraction has increased with heat treatment. Over the data the SFM is plotted with carbon fiber thermal conductivities of 40, 80, 120, and $160 \mathrm{~W} /$ m K respectively. . . . . . . . . . . . . . . . . 117

6.1 VACNT chemical vapor deposition growth chamber. The $100 \%$ argon source is tuned to vary water vapor transported into the system, altering the sample properties. The flow rate of the argon hydrogen mix is varied which also serves to tune the intrinsic properties of the sample. Ethylene serves as the carbon source material, and sublimated ferrocene produces iron catalyst particles.

6.2 (a) Five representative Raman spectra of CNT array Sample D. (b) Ratio of the D and G peak intensities versus position along the CNT array in Sample D. As we move up the array $(1$ to $41 \mu \mathrm{m})$ the ratio increases indicating increased disorder in the tubes. . . . . . . . .

6.3 Transmission electron microscope image of a portion of a carbon nanotube from Sample C. At the widest point the CNT diameter is $25 \mathrm{~nm}$. Within the nanotube is a piece of iron with a $12 \mathrm{~nm}$ diameter at the widest point. It is apparent that the catalyst particle, a remnant from the growth process, alters the nanotubes inner diameter and outer diameter. . . . . . . . . . . . . . . . 
6.4 A $1 \mu \mathrm{m}$ by $1 \mu \mathrm{m}$ TEM image of several carbon nanotubes from Sample C. Throughout the many multi walled tubes, the dark catalyst particles are regularly present (highlighted by white rectangles), often significantly altering the tubes' features. . . . . . . 126 


\section{List of Tables}

3.1 Best Fit Results . . . . . . . . . . . . . . . . . . . 50

4.1 Thermal Testing Results . . . . . . . . . . . . . . . . . 69

4.2 List of All Unique Sample Fabrication Conditions and Their Measured Properties. . . . . . . . . . . . . . . . . . 84

5.1 Fit Parameters of each CNF Sample using a Voigt and Asymmetric Lorentzian for the D and G Peaks, Respectively . . . . . . . . . . . . 102

6.1 Sample Summary (For each sample the variable fabrication parameters are displayed. The flow rate of argon is indicative of the amount of water vapor present during growth. The flow rate of the 9:1 ratio of argon and hydrogen has two stages, the flow prior to ethylene's introduction into the system, and the flow rate once this carbon source has been introduced. The last two columns display the best first thermal conductivity and volumetric heat capacity from $3 \omega$ data which have an uncertainty of $13 \%$ and $17 \%$ respectively.) . . . . 128 


\section{Chapter 1}

\section{Introduction}

\section{$1.1 \quad$ Background}

In 1959, Richard Feynman gave a speach at Caltech to the American Physical Society entitled "There's Plenty of Room at the Bottom." In this visionary speech, Feynman explored the possibilities of what science could achieve by "controlling and manipulating things on a small scale." He went on to discuss the possibilities of arranging atoms as desired with the effects of miniaturizing the computer, building tiny machines, miniaturizing biological systems, and writing information in an area of a few atoms. Much of what Feynman laid out in this talk has come to fruition and is currently being researched to further push the boundaries of what is possible at the smallest length scales. The rapid advancement of devices of micro and nanoscale dimensions has been extraordinary for both the inventions designed as well as the rapid pace in which these new technologies have become commonplace in society. These advances are readily apparent in the mobile miniature computers known as smart phones with transistor dimensions under $30 \mathrm{~nm}$ and continuing to decrease.[5]

A side effect of fabricating nanoscale dimensioned electronics is the requisite control of a device's thermal energy on the same length scale in order to optimize performance. Microelectronics generally require a thermally stable environment to 
perform. Unfortunately, unlike electrical conductivity, which has a tunability of about 20 orders of magnitude, thermal conductivity is currently only tunable up to about 6 orders of magnitude $\left(10^{-3}-10^{3} \frac{\mathrm{W}}{\mathrm{mK}}\right)$ at room temperature.[6] Novel composite material systems consisting of a matrix of nano structured materials open possibilities for tuning a variety of mechanical and thermal properties to produce a unique combination of such attributes which are otherwise unavailable. However, by tuning structural characteristics at the micro and nano length scales, macroscopic models of heat transport often fail to account for the interactions dominating thermal transport.

Traditionally, thermal transport through a material is defined by Fourier's Law:

$$
q=-k \nabla T
$$

where $q$ is the heat flux $\left[\mathrm{W} / \mathrm{m}^{2}\right], \nabla T$ is the temperature gradient $[\mathrm{K} / \mathrm{m}]$ and $k$ is the material's thermal conductivity $[\mathrm{W} / \mathrm{m} \mathrm{K}]$. For macroscale homogenous materials, thermal conductivity is a function of temperature, $k(T)$. However at reduced length scales, when the thermal energy carriers' mean free path, $\lambda$, is similar to or greater than the material's characteristic length, L, the material's thermal conductivity becomes dependent on the reduced length scale, $k(T, L)$. Understanding the effects of reduced length scales on thermal transport, as well as how thermal energy crosses the more frequent interfaces in nano devices is an ongoing area of research.[7] Predicting and understanding thermal transport is further complicated in heterogenous systems in which energy moves between the host material, $h$, and the included material (potentially a geometrically definable particle or pore), $p$. Ultimately thermal conductivity can become a function of the percentage composition of each phase, $\phi$, the structure matrix of the composite material, $S$, as well as variations in each phase's thermal conductivity due to the reduced length scales $\left(L_{H}\right.$ and $\left.L_{P}\right)$ resulting in $k\left(T, L_{H}, L_{P}, \phi, S\right)$ which is still an 


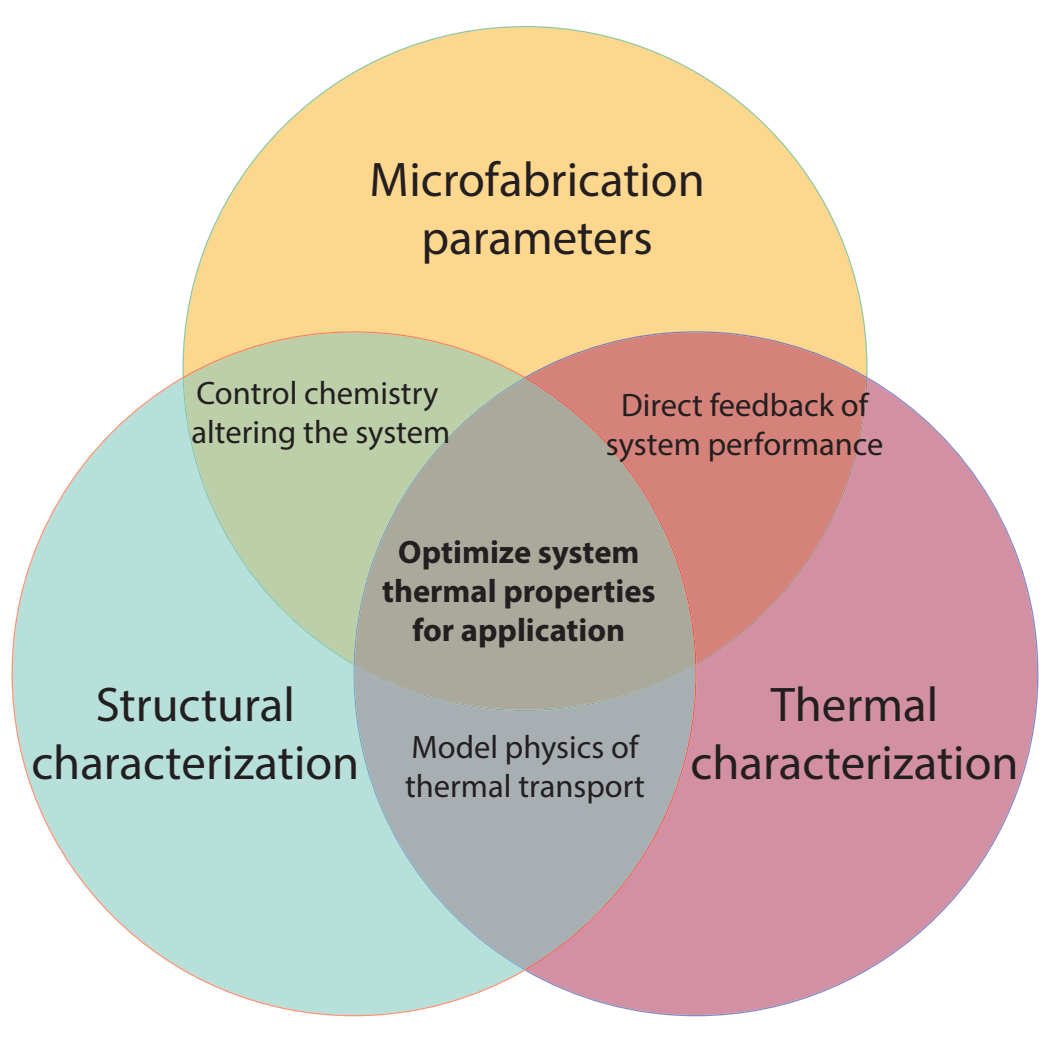

FIGURE 1.1: Scope of optimizing thermal performance.

oversimplification.

However, these parameters describing the structure of the system cannot be tuned in a metaphorical vacuum. Systems containing matrices of more than one material are subject to fabrication conditions which influence a conglomerate of parameters. To optimize the thermal performance of a heterogenous microsystem for a specific application, the fabrication parameters, resulting structure, and thermal properties must all be simultaneously understood. This dissertation aims to advance the path to such understanding. Studies on three unique composite microsystems with specific intended thermal applications are presented with the overarching theme of relating fabrication, structure, and thermal properties (see Figure 1.1). The remainder of this chapter gives a brief overview of specific thermal applications in which control of microscale thermal transport can be used to improve device performance and an outline of the remainder of the dissertation. 


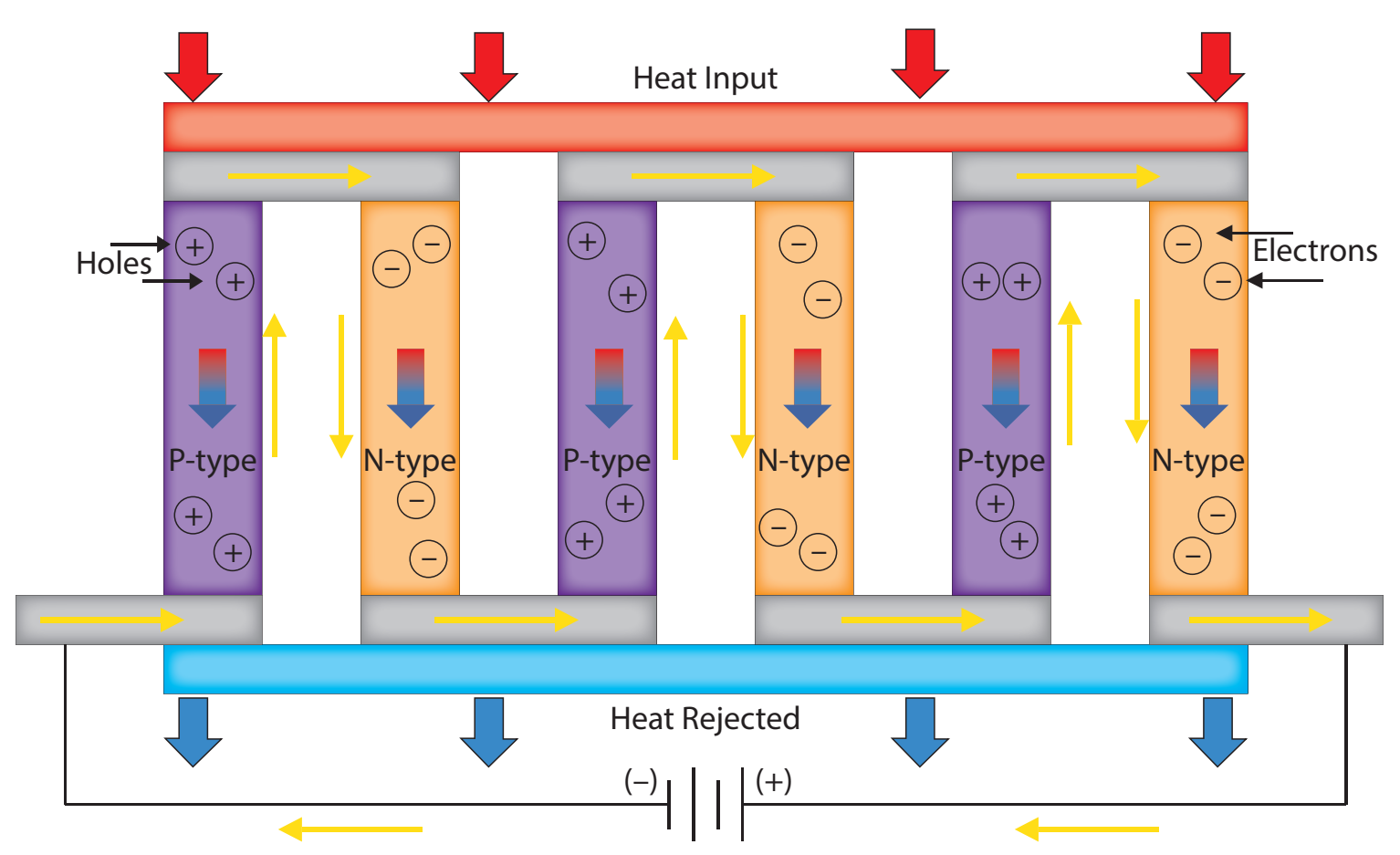

FiguRE 1.2: A thermoelectric generator utilizing the Seebeck effect.

\subsection{Thermoelectric devices}

A strong global push to transition away from nonrenewable energy sources has motivated research into solid state thermoelectric devices (TED). Depending on the application, TEDs take advantage of two separate effects: waste heat (specifically a temperature gradient) can be converted into usable electricity via the Seebeck effect (see Figure 1.2), or the Peltier effect can utilize an induced electrical current to actively cool a surface and remove unwanted thermal energy.

According to annual studies released by Lawrence Livermore National Laboratory, $[8]$ around $60 \%$ of energy generated in the United States is rejected to the environment. Much of this is in the form of waste heat. Several industries are beginning to implement thermoelectric technology to reclaim this energy. Automobile manufactures are currently implementing TED technology utilizing the Seebeck effect to recharge hybrid vehicles; taking advantage of components generating unwanted heat and converting it into electricity used to recharge the 
vehicle's battery. $[9,10]$ Peltier cooling is also being considered in automobiles to replace relatively more complex coolant loops. Key advantages include elimination of the need for refrigerant, reduced noise, shorter thermal equilibrium times, reduced weight, and elimination of some moving parts.[11] Other applications of TEDs actively being pursued include converting waste heat during nuclear decay of radioisotopes into energy to aid in powering space missions[12] and cooling computer systems.[13]

Figure 1.2 shows a thermoelectric device utilizing the Seebeck effect. The device is positioned so that waste heat is deposited near the top of the p-type and n-type semiconducting legs. This diffusion of thermal energy causes a movement of particles towards the cool side: primarily electrons in the n-type material and primarily holes in the p-type material. Therefore, there also is a net movement of electrical current in either material (but in opposite directions). By connecting alternating ends of the doped legs, the electrical current generated can be moved to the rest of the circuit and harvested as usable energy. The dimensionless figure of merit describing each material constituting the thermoelectric device is defined as

$$
Z T=\frac{S^{2} \sigma T}{k}
$$

where $\sigma$ and $k$ are the material's electrical and thermal conductivity. The Seebeck coefficient, $S$, is the change in voltage with respect to temperature. More fundamentally, the Seebeck coefficient is related to asymmetry of the electron distribution around the Fermi energy. To optimize the performance of a thermoelectric, the electrical and thermal conductivity of the material must be tuned independently. This is a unique challenge.

Microstructuring materials opens pathways for independently controlling electron and phonon transport in a material.[14-16] However even complex nano structuring can be counterproductive to increasing $Z T$.[17] In order to optimize a 


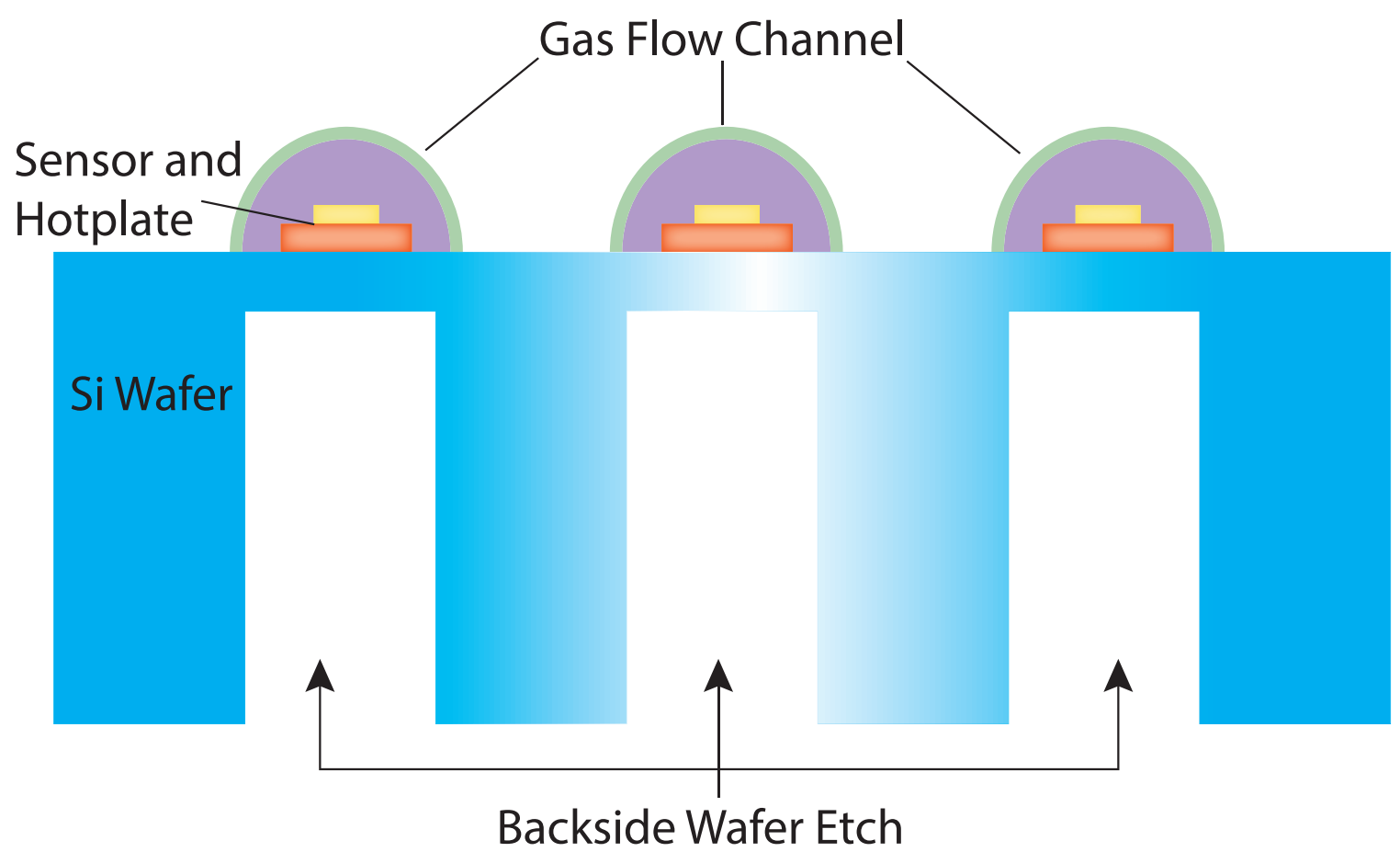

FIGURE 1.3: Diaphragm for thermally isolating hot plates and sensors at elevated temperatures.

thermoelectric device comprised of a specific material system, the physics of both thermal and electrical transport must be sufficiently understood to anticipate the effects of varying the system microstructure through feasible changes in the microfabrication process.

\subsection{Microscale sensors}

Metal oxide gas sensors require operating temperatures ranging from $200^{\circ} \mathrm{C}$ to $500^{\circ} \mathrm{C}$. When using a chip filled with sensors to detect a variety of gases, microhotplates are employed to achieve the desired operating temperatures. Ideally, thermal stability of the device is achieved with temperature uniformity and low power density while occupying the least space possible without sacrificing the structural integrity of the system.[18] To achieve such properties when the ambient temperatures are several hundred degrees below the operating temperatures, 


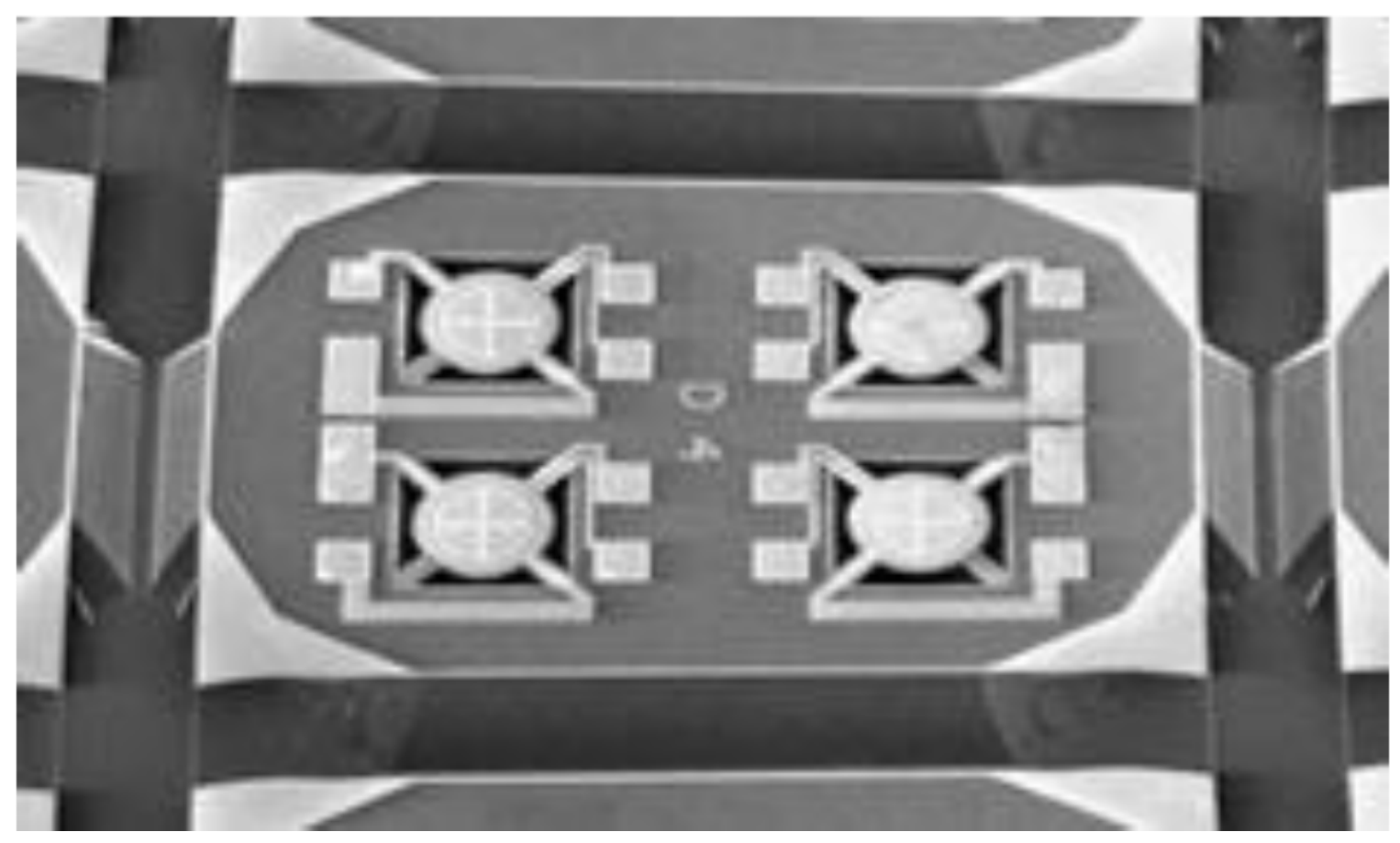

Figure 1.4: Silicon nitride diaphragm isolating hot plates by surface machining developed in collaboration with Honeywell.

thermal insulation with microscale dimensions must be utilized.

Traditionally, a diaphragm sitting above back etched channels on a substrate is utilized to thermally isolate the hotplates from the rest of the wafer as depicted in Figure 1.3 and Figure 1.4. Above these hotplates sits the device of interest, a metal oxide gas sensor, which operates at temperatures well above ambient. This method of thermal isolation has severe thermal, mechanical, and spatial limitations compromising the device's performance. Large gaps on the wafer weaken the structural integrity. To overcome these obstacles, a thin material placed directly below a hotplate which can thermally isolate it from the rest of the wafer while maintaining its structural integrity could be employed. Ideally such a material would be a superior insulator to non-convecting air and be able to directly support the hotplate and sensor. Achieving such properties will require exploring material systems which can achieve thermal conductivity values near that of non-convecting air. 


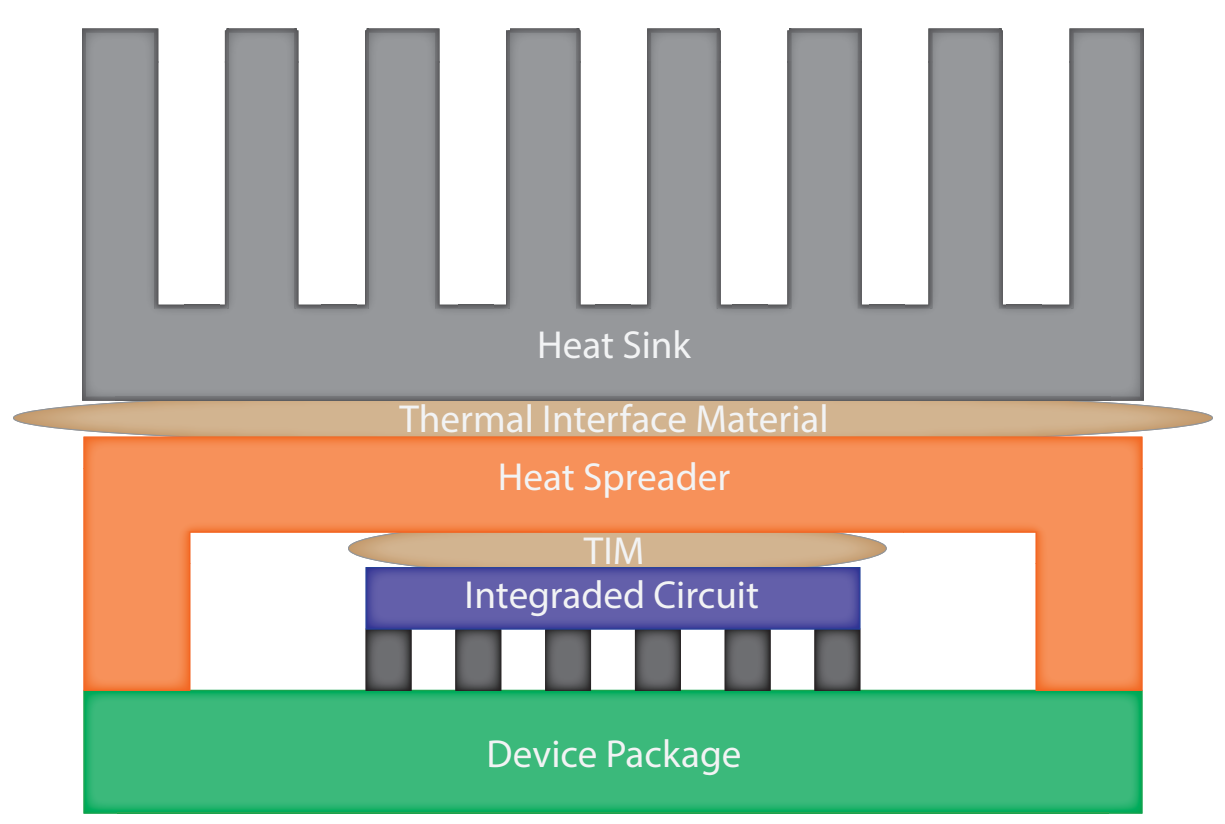

Figure 1.5: Schematic of a thermal interface material aiding in removing heat from a circuit element.

\subsection{Thermal interface materials}

As predicted by Moore's Law, the transistor density on integrated circuits has grown exponentially with time.[19] The increasing power density causes an ever greater amount of waste heat to be produced and unwanted temperature rise in the system often resulting in device failure. Attaching the integrated circuit directly to a heat sink causes a relatively large thermal resistance at the interface. Repeated heating and cooling of materials with mismatched coefficients of thermal expansion causes damage to atomic bonds between the layers eventually resulting in a thermal bottleneck.[20] To overcome this obstacle, a thermal interface material (TIM) must be inserted between the layers (see Figure 1.5). The key TIM properties are high thermal conductance combined with mechanical compliance to prevent atomic bonds from breaking as the materials expand and contract.

To achieve this combination of mechanical and thermal properties, nontraditional material systems must be considered. Many highly conductive materials like diamond offer little compliance and do a poor job of filling 
microscopic impurities of the material surfaces. Traditional TIMs used to fill these voids, such as solders, cannot maintain chemical bonds at the interfaces as the material strains during thermal cycling. Conversely, more compliant materials such as polymers often have poor thermal conductivity. Future generation TIMs must use unique material systems which balance these two properties if the increasing device (and power) density trend of Moore's Law is to continue.

\subsection{Objectives}

This work studies the practical application of heterogenous material systems as key components in thermal management solutions in nanoscale devices. Three such material systems will be studied with a primary focus on understanding and relating their structural attributes to the composite system's thermal conductivity. Such an understanding will aid in optimizing fabrication processes as well as tuning the thermal properties of the microsystem. Focus is also given to considering how to accurately measure the thermal transport in these difficult to characterize systems. These topics are divided into the following chapters:

- Chapter 2 - Thermal Transport in Heterogenous Material Systems This chapter aims to familiarize the reader with thermal transport within material systems. An introduction is given to heat transport in single phase and multi phase systems. Additional complexities that arise due to reduced length scales are also discussed.

- Chapter 3 - Thermal Characterization via $3 \omega$

This chapter reviews the primary technique used to quantify thermal properties in this work: $3 \omega$. Difficulties often overlooked in literature are discussed. A novel bidirectional experimental design uniquely optimized for characterizing material systems studied in later chapters is presented along with initial studies on materials of known thermal conductivity. 
- Chapter 4 - Thermal Isolation via Thin Film Silica Aerogels

The first of the three material systems studied in this work is thin film silica aerogel (TFSA). Recipes for creating thin film aerogels are presented. The challenges and results of micro fabricating on these samples are discussed. TFSAs are shown to be a viable alternative for insulating micro scale thermal devices. Additionally, the intricacies of TFSAs structure are examined and correlated to measured thermophysical properties to provide insight into thermal transport within the material.

- Chapter 5 - Pressure Dependent Thermal Transport in Carbon Nanofiber Networks

Networks of randomly aligned, turbostratic carbon fibers are thermally characterized. Conditions influencing the system's effective thermal conductivity are systematically tuned to gain insight into the fundamental path of heat transport within the carbon network. The unique modification of $3 \omega$ presented in Chapter 3 is employed to obtain the samples' thermal characteristics as a systematically varied pressure is applied. Predictive thermal modeling is employed to elucidate the effects of changing system parameters on the measured thermophysical properties of the system.

- Chapter 6 - Vertically Aligned Carbon Nanotube Arrays for Heat Removal

This chapter explores the thermal conductivity of carbon nanotube arrays potentially used for thermal interface materials. A chemical vapor deposition process is employed and varied to alter the characteristics of the system. Again, using the bidirectional $3 \omega$ system, the thermal properties of a variety of samples are isolated and related to fabrication methods.

- Chapter 7 - Conclusions

A summary of the contributions of this dissertation is presented. 


\section{Chapter 2}

\section{Thermal Transport in}

\section{Heterogenous Material Systems}

\subsection{Overview}

This chapter provides an overview of heat transfer in solids and gases relevant to the studies in this work. Macroscopic heat transfer through each phase is presented followed by the complications created by reducing the characteristic length scale. The interactions that arise in heterogenous material systems are discussed. Elementary heat transfer models to account for these interactions are also presented.

\section{$2.2 \quad$ Single phase solid}

Thermal energy travels through solid media generally by the collisions and diffusion of energy carrying particles due to the presence of a temperature gradient. This is the classic definition of thermal conduction. As stated in Chapter 1, Fourier's law describes the flux of heat through a solid in terms of the temperature gradient, $\nabla T$, and material thermal conductivity, $k$. 


$$
\begin{gathered}
q=-k \nabla T \\
\nabla T=\frac{\partial T}{\partial x} \hat{x}+\frac{\partial T}{\partial y} \hat{y}+\frac{\partial T}{\partial z} \hat{z}
\end{gathered}
$$

In which $\hat{x}, \hat{y}, \hat{z}$, are the unit vectors of the cartesian coordinates.[6] A general kinetic theory derivation considering the individual thermal energy carriers leads to an approximation for thermal conductivity.

$$
k=\frac{1}{3} C \nu \lambda
$$

The volumetric heat capacity of the material, $C$, is the product of the specific heat capacity, (per unit mass) c, and the material density, $\rho$. The energy carrier's group velocity and mean free path are $\nu$ and $\lambda$, respectively. Alternatively, Equation 2.3 can be written in terms of the energy carrier's scattering rate: $\tau=\lambda / \nu$.

The particles (and quasiparticles) which transport thermal energy via conduction are quantized atomic vibrations, electrons, and molecules. We will first consider thermal transport by phonons. A phonon is defined as the minimum energy of a quantized lattice wave that extends through the entire crystal. Phonons dominate thermal transport in crystalline semiconductors (particularly undoped or lightly doped semiconductors).

The mean scattering rate (and therefore the mean free path) of a phonon is understood through Matthiessen's rule.[21]

$$
\frac{1}{\tau}=\frac{1}{\tau_{M}}+\frac{1}{\tau_{U}}+\frac{1}{\tau_{D}}+\frac{1}{\tau_{B}}+\frac{1}{\tau_{E P}}
$$

Phonon scattering exists in several forms, each with a quantifiable scattering rate. The mass impurity scattering rate, $\tau_{M}$, is caused by the presence of different isotopes of a material in an otherwise isotopically pure system.[22] Electron-phonon scattering rates, $\tau_{E P}$, can significantly reduce the mean free path of a system as 
larger voltage biases are applied.[23] Umklapp scattering, $\tau_{U}$, is a type of phonon-phonon collision which results in a phonon scattering into a different brillouin zone. Umklapp scattering becomes becomes dominant at high temperatures.[24] Defect scattering, $\tau_{D}$, is caused by variations from the expected crystalline lattice of the system.[25] Lastly, boundary scattering, $\tau_{B}$, exists at the interface between two materials. Boundary scattering can dominate thermal transport as the characteristic length scale and dimensionality of the system are reduced.[26] Quantifying the change in boundary scattering at reduced length scales is key to anticipating thermal transport in nano structured systems. Due to the frequency dependence of each of these individual scattering terms, it is more accurate to denote the phonon scattering rate as a function of the wave's angular frequency, $\tau(\omega)$, implying the phonon's mean free path is also a function of angular frequency, $\lambda(\omega)$. For any material, a spectrum of phonon frequencies known as the phonon dispersion exists that is a function of the material's phonon density of states. The phonon density of states is defined as the number of quantum states per unit volume per unit frequency that a phonon could occupy.[6] Both the internal energy stored by a phonon and its group velocity are a function of the individual energy carrier's frequency, resulting in $C(\omega)$ and $\nu(\omega)$. Therefore, the kinetic theory model of phonon thermal conductivity in a solid is more accurately written:

$$
k=\int \frac{1}{3} C(\omega) \nu(\omega) \lambda(\omega) d \omega
$$

As the size of the material is reduced, $\tau_{B}$ is decreased, limiting energy transport by phonons. Additionally, reduced sizes can prevent a portion of phonon frequencies from existing, altering each term's frequency dependency and further altering heat transported due to the wave nature of phonons. While quantifying phonon transport from first principles is outside the scope of this work, understanding the root causes of deviations in a material's thermal conductivity from predicted bulk values provides 
insight and intuition into thermal transport as complexities are added to the material system.

Beyond phonons, thermal energy transported through vibrating atoms can also fall into two other categories. When no long range order in the system exists (such as an amorphous solid) the vibrating atoms act as independent oscillators transferring energy directly through collisions with neighboring atoms. Thermal energy transfer through this point scattering is technically not considered transport via phonons due to the lack of crystal symmetry. Only considering these short range vibrations leads to predictions of the lower limit of thermal conductivity which does a reasonable job of predicting thermal conductivity in many amorphous systems.[27-30] Somewhere between these two descriptions of energy transported by vibrating atoms exists fractons, which are defined as the thermal energy carried within fractal structures. The properties of a specific fraction are a function of the system's symmetry and fractal dimension.[31] Fractons exhibit a unique density of states and are sometimes theorized to explain thermal conductivity in amorphous materials. [32, 33]

In metals, generally, an abundance of free electrons contribute significantly to thermal energy transport. The free electrons are often thought of as a gas diffusing thermal energy, traveling until interrupted by some scattering event.[6] Heat conduction by electrons can be considered in a very similar manner to heat conducted by phonons as in Equation 2.5. However the electronic dispersion curve and density of states are generally considered in terms of allowed energies, $E$, rather than frequencies. This leads to

$$
k=\int \frac{1}{3} C(E) \nu(E) \lambda(E) d E
$$

In this instance, $C, \nu$, and $\lambda$ refer to the electronic volumetric specific heat, group velocity, and mean free path respectively. To understand thermal transport within nanostructred system, it is necessary to think of how the unique structure causes 
these energy carrying particles to deviate from bulk, homogenous conditions.

\subsection{Single phase gas}

In a classic macroscopic view of heat transfer, thermal energy moves in a gas primarily through convection: the collective movement of groups or aggregates of molecules within the fluid. Put another way, heat is being moved through mass transfer. However, randomly moving particles will still collide and transfer energy through diffusion even within a bulk of moving fluid, leaving a path for conduction. In infinite length scale scenarios this conduction is often negligible. The Rayleigh number measures the relative strength of conduction and convection. For free convection near a boundary:

$$
R a=\frac{g \beta \Delta T L^{3}}{\nu \alpha}
$$

Here $\beta$ is the fluid's coefficient of thermal expansion, $g$ is acceleration due to gravity, $L$ is the limiting length scale dimension, $\nu$ is the kinematic viscosity, and $\alpha$ is the fluid's thermal diffusivity.

The Rayleigh number is strongly dependent upon the length scale a fluid particle can travel before colliding with the boundary. When the Rayleigh number is below a critical value, then conduction dominates heat transfer and convection can be considered negligible. Turcotte and Oxburgh determine this critical Rayleigh number to be 657.[34] Clyne et al. have demonstrated that at atmospheric temperature and pressure natural convection is insignificant for pore sizes under 10 mm diameter.[35] This length scale is several orders of magnitude larger than pore sizes of the material systems considered in this work. The aerogel films considered in Chapter 4 are under $1 \mu \mathrm{m}$ total thickness, and the studied CNT arrays in Chapter 6 are less than $100 \mu \mathrm{m}$ thick. While the carbon fibers discussed in Chapter 5 have a total thickness of several centimeters, the effective pore size of fiber 
composites of random orientation can be approximated.[36] For the case of the fibers considered of near $100 \mathrm{~nm}$ fiber radius, this pore diameter is under $1 \mu \mathrm{m}$ in all scenarios tested. Therefore, convective energy transport can be ignored in the studies presented in subsequent chapters.

Even when convective energy transport is negligible, conduction through the gaseous phase still plays a critical role. Conduction occurs in gases by the diffusion of thermal energy via the collision of molecules. The Knudsen number is useful for considering regimes of gaseous thermal conductivity:

$$
K n=\frac{\lambda_{B}}{L}
$$

Subscript $B$ refers to the fluid particle's mean free path in a bulk, infinitely large medium. As the limiting length scale $L$ decreases (such as a pore's diameter) the Knudsen number increases. The mean free path of a gas in an infinitely large medium is limited by collisions between molecules. The mean free path is therefore a function of the molecule's collision area and distance a molecule moves before colliding with another moving molecule:[37]

$$
\lambda=\frac{m}{\pi \sqrt{2} \rho d^{2}} .
$$

The thermal conductivity of air can be divided into 3 regimes according to the Knudsen number. At $\mathrm{Kn}<0.01$ the gas exists in the continuum regime. Here, air has a thermal conductivity of $0.026 \mathrm{~W} / \mathrm{m} \mathrm{K}$ at atmospheric temperature and pressure as predicted by kinetic theory. When Kn $>10$ the free molecule regime describes thermal transport and the gas thermal conductivity is inversely proportional to the Knudsen number.[38] Between these two regimes exists the transition regime where thermal transport is a convolution of the continuum and free molecule regimes.[39]

In porous materials with very large thermal conductivities, air has a relatively small contribution to thermal conductivity and is often ignored. However, in systems with extraordinarily low thermal conductivities, air plays a non negligible role in 
conduction and, as shown in Chapter 5, can more than double a system's effective thermal conductivity.

\subsection{Heterogenous material systems}

When considering thermal transport in a two material medium, it is common to refer to the one which is continuous throughout the system as the host phase $(h)$ and the included material dispersed throughout the system as the particle phase. Alternatively the included material can be a void containing a fluid which is often called a pore. The included particle or pore $(p)$ often has a definable size and shape. However this may be no more specific than a globular shape with a specified effective radius. It is readily apparent that the effective thermal conductivity, $k_{e}$, is a function of the thermal conductivity of each material, $k_{h}$ and $k_{p}$. In some limiting scenarios in which thermal energy moving between the two respective phases may be negligible, a simple rule of mixing can approximate the effective thermal conductivity:

$$
k_{e}=k_{p} \phi+k_{h}(1-\phi)
$$

in which $\phi$ is the volume percentage occupied by the included particle. Equation 2.10 may be applicable to a system containing vertically aligned rods. This scenario is very analogous to electrical resistors acting in parallel.

However, to consider two phase heat transfer in a wider array of scenarios, shortcomings of Equation 2.10 must be addressed. Suppose the particle phase is anything other than rods aligned in the direction of heat transfer. Instead they are randomly distributed spheres. The thermal contact resistance between the host and particle (or pore), $R_{h p}$, must be considered in the thermal circuit, as well as the frequency of this interaction. Additionally, spheres may be in contact with other spheres but not chemically bonded. Another path of heat flow and another contact resistance, $R_{p p}$, would have to be accounted for. Each of these contact resistances is 
a function of the intrinsic properties of each phase as well as the interface which separates them. Additionally, by including particles throughout the host material separated at distances similar to or less than the host's bulk mean free path, $\lambda_{h}$, then the host phase conductivity, $k_{h}$, will need to be determined as it varies from bulk values. Similarly, the included particles' thermal conductivity may be reduced if its diameter is less than its bulk mean free path, $\lambda_{P}$. Lastly, assuming one can determine $k_{h}\left(\lambda_{h}\right), k_{p}\left(\lambda_{p}\right), R_{p p}$, and $R_{h p}$ (which is far from trivial in many scenarios), a thermal model using these terms as inputs needs to be determined that sufficiently well describes the structure of the medium.

Nan et al. developed a general framework for describing a two material matrix as an effective medium.[40] Here, a Green function is used to describe a homogenous medium representative of the composite to solve for the temperature gradient. By considering the shape of the included particles and assuming these particles are isolated and do not interact, solutions for the effective thermal conductivity of the system are determined. For the scenario of spherical particles like that given above, Nan et al. determine

$$
k_{e}=k_{h} \frac{k_{p}(1+2 \alpha)+2 k_{h}+2 \phi\left[k_{p}(1-\alpha)-k_{h}\right]}{k_{p}(1+2 \alpha)+2 k_{h}-\phi\left[k_{p}(1-\alpha)-k_{h}\right]}
$$

where $\alpha$ takes into account the boundary resistance between the two phases. Others have worked to refine this idea by taking into account the boundary resistance between the particles and host material[41] as well as the resistance between two separate portions of the particle phase.[42] In any case, the accuracy of modeling thermal transport through the system is limited by the understanding of the phases' intrinsic properties, and the accuracy of the thermal model employed. 


\subsection{Thermal resistance at an interface}

The thermal resistance at an interface is often macroscopically thought of in terms of a thermal contact resistance. This thermal contact resistance exists due to the imperfections of the surfaces pressed together (often filled with microscopic voids) and varies with applied pressure between the two materials. But even at an atomically perfect interface between two dissimilar materials, a temperature gradient exists known as the Kapitza resistance, or just thermal boundary resistance.[43, 44] The mathematical treatment of both types of interfacial resistances, $R$, is the same:

$$
Q=\frac{\Delta T}{R}
$$

Methods exist for measuring the temperature drop at a single interface in a well defined system and determining its thermal boundary resistance, particularly in thermal flow between planar layers. However, in two phase systems with many interfaces spread throughout the material, it is not possible to directly measure the interface resistance either between two of the included particles or between a particle and host. Therefore it is necessary to depend on modeled interface resistance as an input to predicting the effective thermal conductivity of a composite system. Assuming diffuse scattering at a bonded interface, Chen determined[45]

$$
R \approx 4 \frac{C_{1} \nu_{1}+C_{2} \nu_{2}}{C_{1} \nu_{1} C_{2} \nu_{2}}
$$

Where subscripts 1 and 2 refer to each material on either side of the interface and $C$ and $\nu$ are the material's specific heat capacity and the group velocity of the energy carriers, respectively. Understanding the resistance to heat flux at an interface is an ongoing area of research. There are many models, and many proposed modifications to existing models, that all attempt to predict thermal boundary resistance.[45-50] In 
microstructured systems, with a high interface density relative to bulk systems, this resistance to thermal flux plays an increased role. It can be difficult to deconvolve the effect of thermal interface resistance from the reduced thermal conductivity of a material due to size reductions within a specific material.

\subsection{Summary}

The three material systems studied in this dissertation: thin film silica aerogels, carbon nanofiber composites, and vertically aligned carbon nanotube arrays each have a broad range of potential thermophysical properties. Predicting how varying the structure of these materials will influence thermal transport requires a fundamental understanding of how thermal energy is transported in each system. This chapter has introduced the broad topics of thermal transport in solids and fluids at reduced length scales, and the interactions between them. Relevant textbooks provide much

more detail about each of these topics. $[6,51,52]$ In Chapters 4,5 , and 6 a depth of understanding is added to these areas as necessitated by the system studied. 


\section{Chapter 3}

\section{Thermal Characterization via $3 \omega$}

This chapter provides a background and overview of the experimental methods used in this work in order to aid in understanding and give confidence to the results in the subsequent chapters. The chapter section reviews the third harmonic experiment for measuring thermophysical properties $(3 \omega)$, providing details of the implementation of this system used in this work (which was performed in collaboration with Michael Fish).[53] Next, a unique modification of the experiment designed specifically to measure samples studied later in this work is presented. This method is shown to be generally applicable to a large range of material systems.

\subsection{Review of third harmonic experiments}

The $3 \omega$ technique has been shown to be an accurate method for measuring the thermophysical properties of a material of interest. $[54,55]$ In general a metal line heater (which also serves as a thermometer) has an alternating current passed through it, $I_{1 \omega}$, at angular frequency $\omega$. This current causes a second harmonic Joule heating event: $Q_{2 \omega}=I_{1 \omega}^{2} R_{0}$ in which $R_{0}$ is the electrical resistance of the wire at the ambient temperature. The resulting second harmonic temperature rise, $T_{2 \omega}$, is a function of the thermophysical properties of the heating line and its surrounding. The thermoresistive 
response of the wire, $\frac{d R}{d T}$, can be determined by temperature ramping the system. Thin metal lines have a linear thermoresistive response in temperature regimes in which they do not undergo a phase change. Therefore, the temperature rise can be related to a second harmonic electrical resistance oscillation, $R_{2 \omega}$. By Ohm's law, $R_{2 \omega}$ and $I_{1 \omega}$ can be used to relate the properties of the system to the measurable third harmonic voltage drop, $V_{3 \omega}$, from which the experimental method receives its name.[54, 55]

Early modulation techniques to determine thermophysical properties can be traced back over 100 years to the work of Corbino in the field of calorimetry.[54, 56] Corbino's work is distinct from other works at the time due to the dependency of the measured temperature oscillations on the heat capacity of the sample.[57] Russian scientist Filippov measured the third harmonic response of a metal heater surrounded by a liquid to simultaneously determine the thermal conductivity and heat capacity of the liquid of interest.[58] Rosenthal demonstrated how to determine the thermal time constant of a material system by isolating the third harmonic voltage with a bridge circuit design. [59] Holland continued the advancement of the circuit design in a study on the specific heat of titanium.[60]. Birge and Nagel used a $3 \omega$ method as a specific heat spectrometer to determine the frequency dependency of a liquid's heat capacity.[61, 62] Jung and collaborators advanced this idea to create an automated circuit designed to measure the static and dynamic specific heat of a system over an extended frequency range.[63] Moon and Jeong then demonstrated how to determine the dynamic specific heat as well as the thermal conductivity of a liquid by varying the width of the heating strip.[64] Birge et al. have presented a review of specific heat spectroscopy via $3 \omega \cdot[65]$

In the late 1980's and 1990's 3 $\omega$ techniques began receiving widespread usage to measure thermophysical properties of solids with a thin metal line directly adhered to the material system of interest. A series of studies by Cahill and collaborators measured the thermal conductivity of amorphous solids with a simplified circuit design and simplified analysis able to determine a material's thermal conductivity over a 
limited frequency range.[55, 66-68] More recently, focus has turned to determining the properties of a thin film deposited on a substrate. Lee and Cahill studied the effects of thin film deposition process on the thermal conductivity of $\mathrm{SiN}_{x}$ and $\mathrm{SiO}_{2}$ films on Si substrates.[69] Other work continued to focus on unique attributes of thin films based on film size and deposition methods.[70] Bullen et al. performed a similar study on a wide variety of amorphous carbon films showing a variation of a full order of magnitude in thermal conductivity.[71] $\mathrm{Hu}$ et al. studied silica xerogel films with $48 \%$ porosity and found an order of magnitude reduction from bulk silica.[72] These results were confirmed by others using $3 \omega$ in a similar scenario on porous xerogel/aerogel films at similar densities.[73]

Several publications using $3 \omega$ focused on presenting methods to solve the heat diffusion equation for a thin heater above any number of planar films of varying thermophysical properties.[74-78] Other works have demonstrated the ability to use the material of interest as the heating wire.[79-83] These experiments isolated the specimen from the surroundings and simultaneously determine the wire's time constant as well as its thermal conductivity and heat capacity.

\section{$3.23 \omega$ system implementation and measurement considerations}

Figure 3.1 shows a common multilayer $3 \omega$ sample. Above the planar films on the sample are 4 contact pads which are connected to the central heating element. The oscillating current passes into the sample through the lower left pad and exits through the lower right pad. The upper pads are used to simultaneously detect both the first harmonic and third harmonic voltage drop across the central heating wire. In our implementation the sample is physically placed within a cryostat and connected to the rest of the circuit by means of metal pins lowered to make contact with each pad. Care must be taken to avoid puncturing the $200 \mathrm{~nm}$ thick pads when contact is 


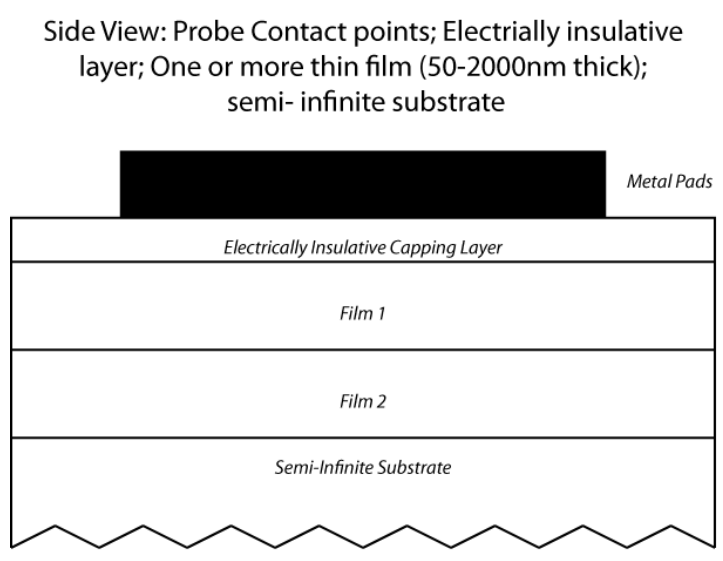

Top View: four metal pads connected by a $200 \mathrm{~nm}$ thick wire serve as a heat source and thermom-

Side and Top View of a Three Omega Sample

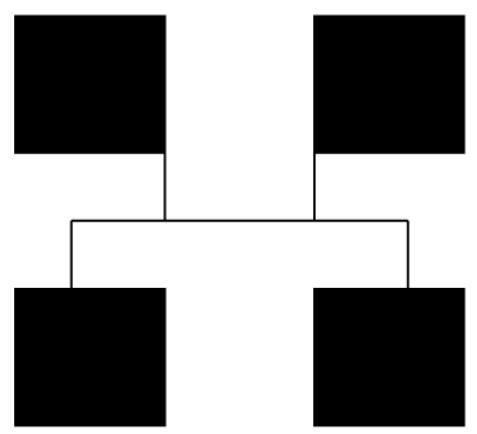

Figure 3.1: Traditional sample characterized via the $3 \omega$ technique. The four contact pads serve as a four point probe connection allowing a voltage reading to be made on the central portion of the thin heating wire without significant interference from the remainder of the electrical circuit.

made in this manner. On samples in which puncturing the pad could not be avoided, such as the aerogel samples discussed in Chapter 4, larger graphite rods were directly lowered onto the pads. These pins (or graphite rods) were connected directly into the wiring of the cryostat, which was then routed to the remainder of the electrical circuit. Challenges of making electrical connection with the sample are discussed in greater detail in Fish's Thesis. [53]

Since the advent of turn-key lock-in amplifiers, the equipment and circuit design of $3 \omega$ type experiments has been greatly simplified. Generally the lock-in amplifier is able to serve as a frequency generator, which drives the AC signal over the sample. Typical equilibration times for the system are around 5-10 signal envelopes, meaning the data can be determined nearly instantaneously at most frequencies. The $1 \omega$ driving voltage will inherently create noise in the $3 \omega$ voltage measured. This noise is typically orders of magnitude greater than the signal one seeks to measure. To account for this, additional electrical resistance is placed in the experimental circuit. The current generated, $I_{1 \omega}$, passes over both elements in the circuit, with both regions set to have the same steady state electrical resistance, $R_{0}$. Each path is read by the 
lock-in amplifier through inputs A and B respectively. The lock-in amplifier reports the A-B difference in $V_{3 \omega}$ from each path. Ideally, the only signal difference that remains is caused by the influence of the thermoresistive response of the heating element on the sample.

Two common circuit designs to subtract noise in the third harmonic signal are often implemented in the literature: the Wheatstone bridge method $[63,70]$ and a series resistor method $[66,76]$. For this work, both circuit designs were assembled and tested. The Wheatstone bridge method ensures that the voltage source more closely resembles an ideal current source, which is the assumption made when relating the signal to the temperature rise in the sample.[84] However, in this circuit configuration relatively smaller percentage of the overall first harmonic voltage drop occurs on the sample heating wire (instead of occurring on other resistors in the circuit). In turn, a smaller temperature rise occurs, and a smaller $V_{3 \omega}$ signal is available to be measured. In this work, an SR830 lock-in amplifier by Stanford Research Systems was used. This device generates a maximum voltage signal of $5 \mathrm{~V}$ which effectively sets the maximum temperature rise $\left(T_{2 \omega}\right)$ which can be created at a given frequency on a given sample for a given circuit design. In all scenarios tested in this work $T_{2 \omega}$ peak temperature rise never exceeded $6 \mathrm{~K}$ and was often less than $1 \mathrm{~K}$. Designs using the Wheatstone bridge circuit generated signal nearly an order of magnitude less than the series resistor circuit. In practice, it was found that this decrease in signal relative to the noise within the system was too large. Analysis of the experiment demonstrated that the decrease in signal to noise magnitude of $V_{3 \omega}$ generated significant error. For this reason, a series resistor method was used.

The circuit design used in this work is depicted in Figure 3.2 and the equipment layout in Figure 3.3. The signal generated by the lock-in amplifier passes through a potentiometer and the sample. Differential amplifiers are used to allow the lock-in amplifier to reference each voltage drop to the system's ground. They serve the dual purpose of protecting the lock-in amplifier inputs from damage. Digital multimeters 


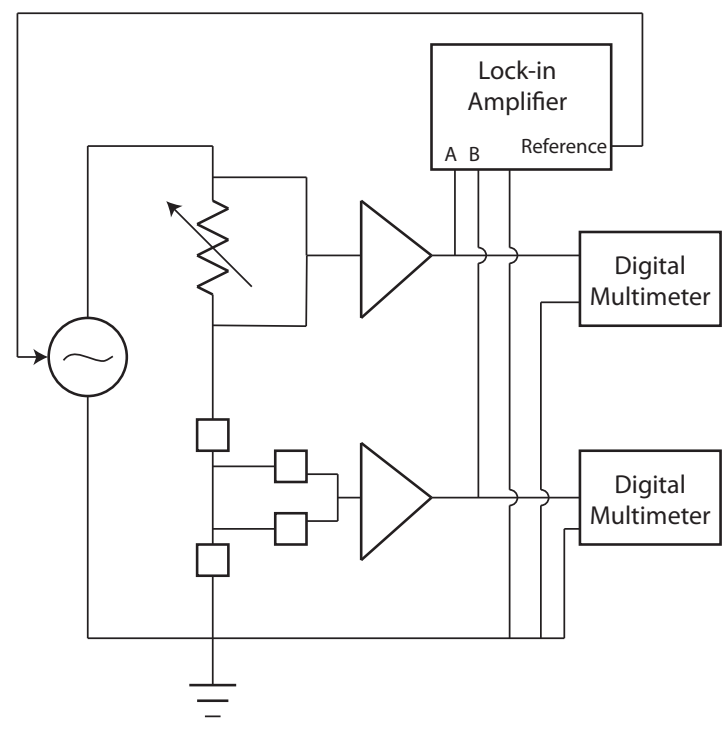

Figure 3.2: Depiction of a simplified $3 \omega$ circuit. Current passes from the lock-in amplifier through the potentiometer, through the sample heating wire, and is then grounded. Digital multimeters and the lock-in amplifier are connected to each element through a differential amplifier. The multimeters are used to determine the first harmonic voltage drop across each element. These readings are used to determine the sample's electrical resistance (and therefore the amount of heat deposited into the sample). The A and B inputs on the lock-in amplifier read the third harmonic voltage drop across each element. The difference in these values is calculated and used to determine the second harmonic temperature rise in the heating wire and ultimately the sample's thermophysical properties.

are placed at the same location as each lock-in amplifier reading to aid in matching $V_{1 \omega}$ drop over each resistance (sample and series potentiometer) and to determine the resistors' electrical resistance, $R_{0}$. The A-B reading on the lock-in amplifier is used to isolate the third harmonic voltage generated by the sample. Data was acquired at driving frequency, $f$, generally ranging from $1 \mathrm{~Hz}$ to $10 \mathrm{kHz}$ with 41 data points collected over this range. In scenarios in which low frequency data was not necessary, the lowest data point collected was at $10 \mathrm{~Hz}$.

To evaluate the circuit, an electrical resistor was substituted for the sample, and the potentiometer was set to match its resistance. Ideally no element within the circuit has a thermoresistive response, therefore any remaining third harmonic signal is noise or system artifacts which must be eliminated or accounted for. A series of tests were performed measuring the first harmonic and third harmonic response of each resistor individually over a wide frequency range. Then the response was tested 


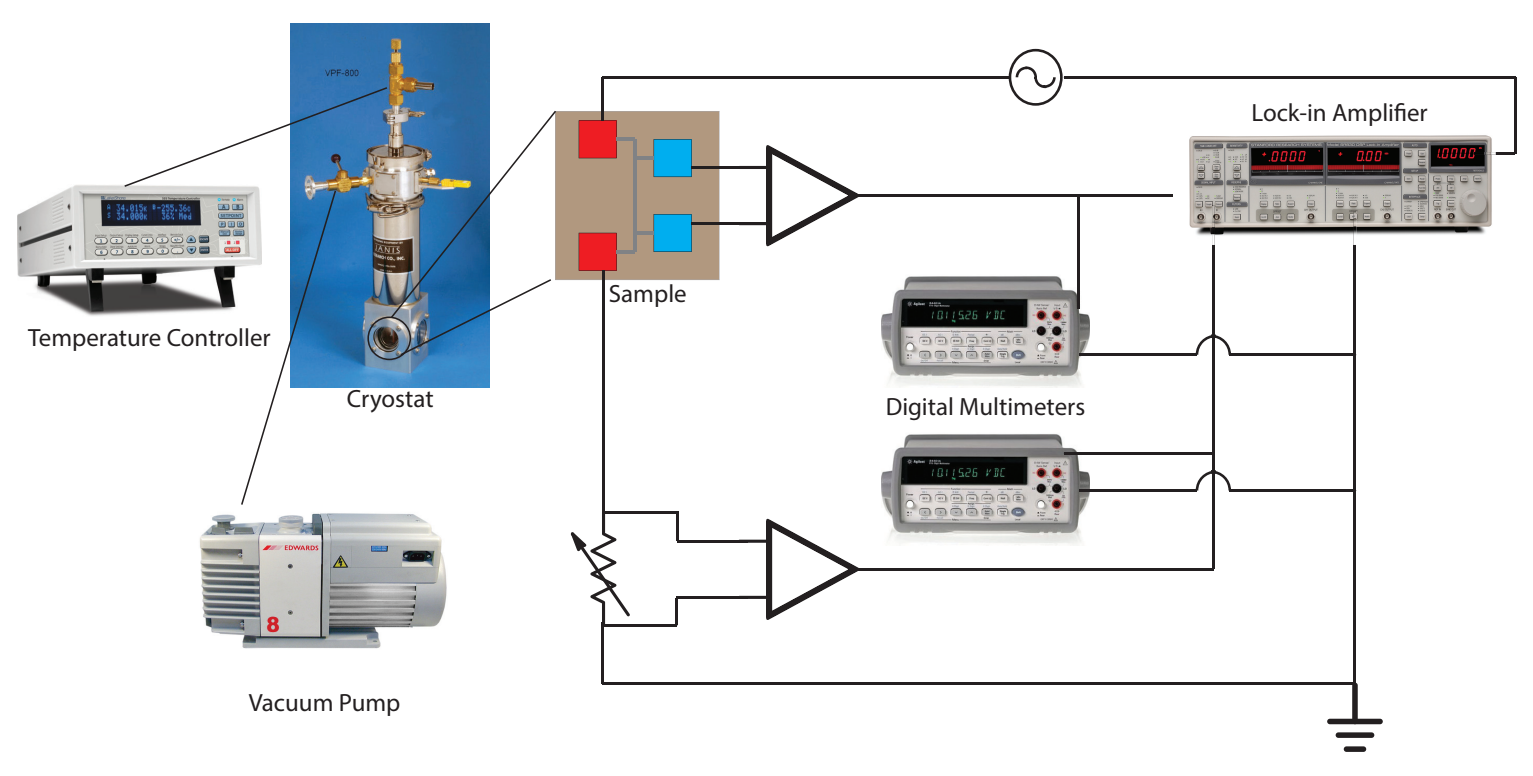

FiguRE 3.3: Schematic of $3 \omega$ equipment layout.

with the circuit in A-B mode (and flipped into B-A alignment). This series of tests allowed for the isolation of portions of the circuit creating artifacts in the measured data. Flaws found included simple problems such as circuit connections which needed to be tightened or portions of the circuit which were not $100 \%$ electrically isolated. Other errors that became apparent included an electrical connection between the cryostat to the metal enclosure.

One key obstacle which came to light during this testing required more than fixing electrical connections to overcome. Figure 3.4 shows the remaining A-B difference after all other circuit corrections were made with the test resistor in place of the sample. Ideally $V_{3 \omega}$ should be zero at all frequencies in this figure. For the in-phase output, this is the case between about 0.5 and 5,000 Hz. However a deviation exists at higher and lower frequencies. This deviation is even more evident in the out-ofphase voltage (the imaginary portion of the $V_{3 \omega}$ signal). For many other flaws found in the circuit, switching the order the current passes either circuit leg (and the order signal's A and B are read) would show an equal and opposite effect. However, for this remaining signal, this was not the case.

Tian found a similar problem and recorded it in her Ph.D. dissertation. [85] The 


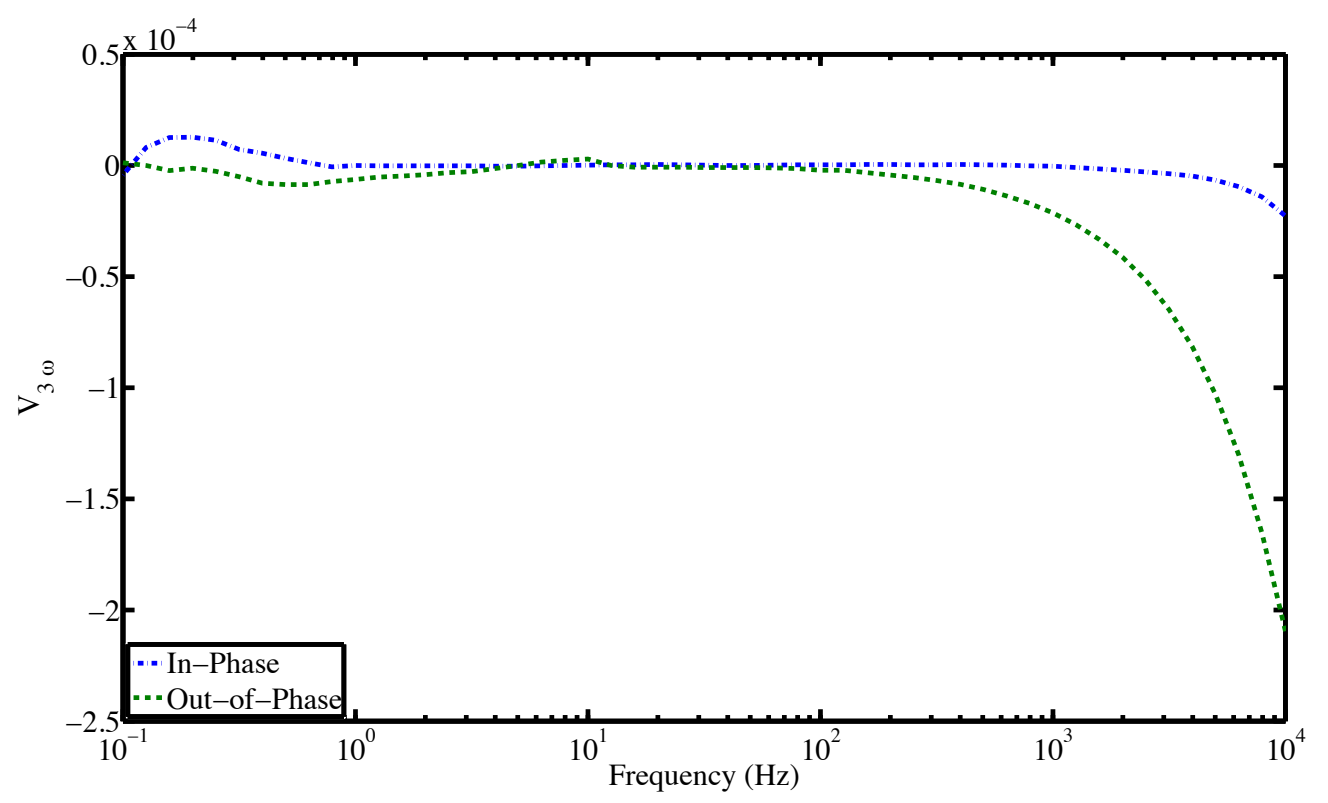

Figure 3.4: Plot of third harmonic voltage with a normal resistor in place of the sample in the $3 \omega$ circuit. Ideally this data is zero at all points. Deviation from zero is insignificant over most of the frequency range for the in-phase signal.

artifact must be caused by frequency dependent impedance existing in the circuit: capacitances and inductances. To overcome this obstacle, Tian modeled the frequency dependent result in her test circuit (with a resistor lacking a thermoresistive response in place of the sample) and subtracted the result from her data. We follow the same methodology using the data from Figure 3.4. In practice, this data correction can be avoided by maintaining frequency ranges where this unwanted signal does not significantly alter the measured data. For our purposes this range is between 0.5 and 5,000 Hz. Most data sets in this work include a few data points between 5,000 and 10,000 Hz. In these situations this data correction was used on the final data points which represent a small fraction of the total data points collected. Without this correction the third harmonic signal would seemingly become negative which should not be physically possible. On first pass, all data in this work was analyzed with and without the correction for both the in-phase signal only then the out-of-phase signal only (four independent fits of a single scan). The 
results of the in-phase signal fits (with and without the correction) were generally within 1-2\%, within experimental uncertainty. This was not the case in some scenarios where a key parameter was most sensitive at high frequencies. Specifically, in some scenarios the correction was necessary to accurately determine the contact resistances discussed later in this chapter, as well as in Chapters 5 and 6. Fitting out-of-phase data is not a common practice as the signal is smaller (and therefore has relatively poor signal to noise ratio) compared to the in-phase data. Comparing best fit results of the corrected out-of-phase-data with both sets of in-phase fits (corrected and uncorrected) generally resulted in decent agreement. However the out-of-phase, uncorrected data varied greatly. The onset of this frequency dependent error at just over $1000 \mathrm{~Hz}$ makes this apparent. All data reported is based on best fits of the in-phase data which contains this impedance correction.

\subsubsection{Determining a sample's thermophysical properties from $V_{3 \omega}$}

The following section discusses the methodology shown by other researchers for extracting thermophysical properties from the $3 \omega$ technique.[68, 74] The root mean square third harmonic voltage drop across the heating line can be related to its temperature rise:

$$
V_{3 \omega}=2 T_{2 \omega} \frac{V_{1 \omega}}{R_{0}} \frac{d R}{d T}
$$

As previously stated many works have presented methods to relate $T_{2 \omega}$ to the thermophysical properties of the system. The solution to the stratified medium partial differential equation for heat flow with an alternating current heat source was solved by Carslaw and Jaeger.[86] Feldman's intuitive implementation of this solution is used throughout this work to solve for the thermophysical properties of multilayer systems. $[74,87]$ The ac temperate rise average over the heating wire is 


$$
T_{2 \omega}=\frac{P}{2 \pi l b^{2}} \int_{0}^{\infty} \frac{B^{+}+B^{-}}{A^{+} B^{-}-A^{-} B^{+}} \frac{\sin (m b)}{\gamma_{h} m^{2}} d m
$$

The heater width is $b$, its length is $l$, and the input power is $P$. Subscript $h$ refers to the heating layer and $m$ is the integration variable. This solution assumes the heating element is infinitely thin, which is reasonable for frequencies with long penetration depths compared to the heater thickness. The terms $A^{+}, A^{-}, B^{+}$, and $B^{-}$are determined by relating the temperature at points throughout the material system. More specifically they represent the pairs of counter propagating waves on either side of the heat source traveling through the system. For any layer $j, \gamma_{j}^{2}=k_{j}^{2}\left(m^{2}-\right.$ $\left.i \omega / D_{j}\right)$ where $k_{j}$ and $D_{j}$ are the layer's thermal conductivity and thermal diffusivity, respectively. One of the sample properties of interest, the volumetric specific heat $\left(C_{j}\right)$, can be extracted from the thermal diffusivity: $D_{j}=\sqrt{k_{j} / C_{j}}$. In between each layer exists interfaces labeled $i$ assigned the equivalent number as the material below the interface. For a single material layer, the temperature distribution is[87]

$$
T(z)=T_{j}^{+} \exp \left(u_{j} z\right)+T_{j}^{-} \exp \left(-u_{j} z\right)
$$

where $T_{j}^{+}$and $T_{j}^{-}$are complex constants conveying the temperature rise at any position in the system. The thermal wave vector is defined as $u_{j}=\sqrt{m^{2}-i \frac{\omega}{D_{j}}}$. The temperature vector is

$$
\widetilde{T}=\left(\begin{array}{c}
T_{j}^{+}(z) \\
T_{j}^{-}(z)
\end{array}\right)
$$

For a layer of thickness $d_{j}$ above the heat source, the temperature on the bottom of the layer can be expressed in terms of the temperature on the top of the layer: [87]

$$
\left(\begin{array}{c}
T_{j}^{+}(0) \\
T_{j}^{-}(0)
\end{array}\right)=\left(\begin{array}{cc}
\exp \left(u_{j} d_{j}\right) & 0 \\
0 & \exp \left(-u_{j} d_{j}\right)
\end{array}\right)\left(\begin{array}{c}
T_{j}^{+}\left(d_{j}\right) \\
T_{j}^{-}\left(d_{j}\right)
\end{array}\right)
$$

For a layer below the heat source, in which thermal flux is traveling in the negative 


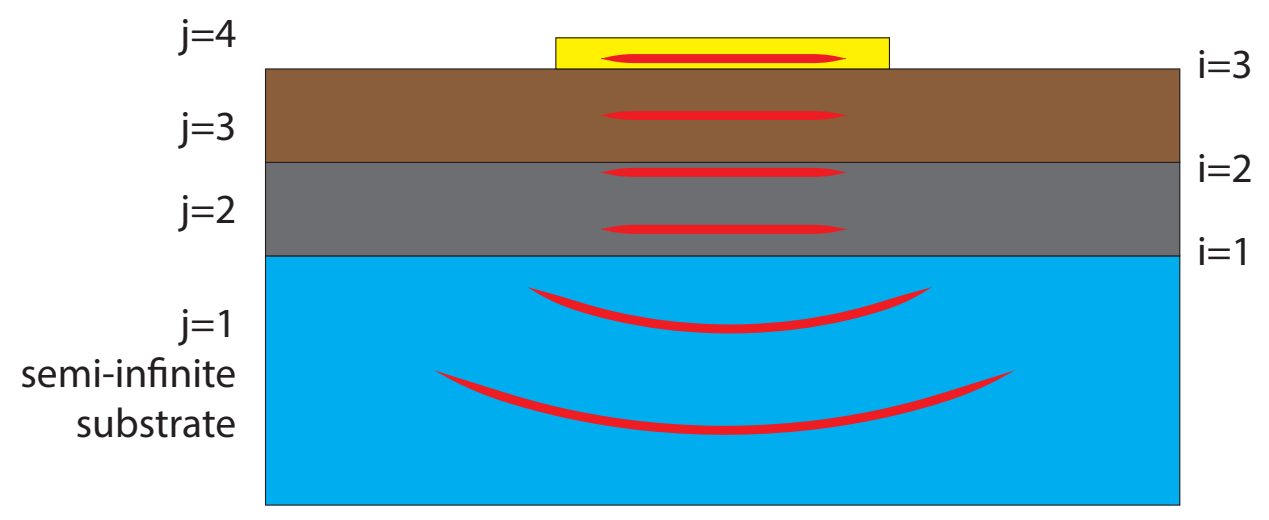

FiguRE 3.5: Depiction of thermal waves traveling from the heating layer into a three layer system including a semi-infinite substrate. In this configuration the system is generally under vacuum. It is assumed all of the heat leaving the heating wire $(j=4)$ is deposited into the top film.

$z$ direction, the sign in the two exponents of Equation 3.5 are switched. The temperature change across some interface, $i$, above the heat source, between layers $j$ and $j+1$ is [87]

$$
\left(\begin{array}{c}
T_{j}^{+}\left(d_{j}\right) \\
T_{j}^{-}\left(d_{j}\right)
\end{array}\right)=\frac{1}{2 \gamma_{j}}\left(\begin{array}{cc}
\gamma_{j}+\gamma_{j+1}+\gamma_{j} \gamma_{j+1} R_{i} & \gamma_{j}-\gamma_{j+1}-\gamma_{j} \gamma_{j+1} R_{i} \\
\gamma_{j}-\gamma_{j+1}+\gamma_{j} \gamma_{j+1} R_{i} & \gamma_{j}+\gamma_{j+1}-\gamma_{j} \gamma_{j+1} R_{i}
\end{array}\right)\left(\begin{array}{c}
T_{j+1}^{+}(0) \\
T_{j+1}^{-}(0)
\end{array}\right)
$$

The thermal boundary resistance at an interface is $R_{i}$, known as the Kapitza resistance. For atomically bonded layers, this resistance is relatively small. For layers pressed together, this thermal contact resistance is much larger. In general, $3 \omega$ experiments are not sensitive to the Kapitza resistance of atomically bonded interfaces. The temperature drop resulting at such interfaces is negligibly small compared to temperature change over the total distance traveled by the thermal waves even in the relatively high frequency range of $10 \mathrm{kHz}$. At atomically bonded surface, where the variation in signal caused by the Kapitza resistance is negligibly small within the total signal, $R_{i}$ will be ignored.

Figure 3.5 shows a metal heating wire (layer 4 ) above two thin films and a semiinfinite substrate. Such a scenario would be necessary to test the thermal properties 
of a metallic thin film that requires an electrically insulative layer between it and the heating wire. For this configuration, $A^{+}$and $A^{-}$will represent the components of the temperature vector above the heating line. When the experimental measurements are taken in a vacuum an insulating boundary condition is applied and each term is equal to $1 / 2 .[74]$ To determine $B^{+}$and $B^{-}$, the components of the temperature vector below the heating line, Equations 3.5 and 3.6 must be successively applied and solved for each material layer and interface the thermal waves pass through. Additionally, a semi-infinite boundary condition can be applied at the backside of the bottom substrate: $B_{1}^{+}=0$ and $B_{1}^{-}=1$. With this information $B_{4}^{+}$and $B_{4}^{-}$become

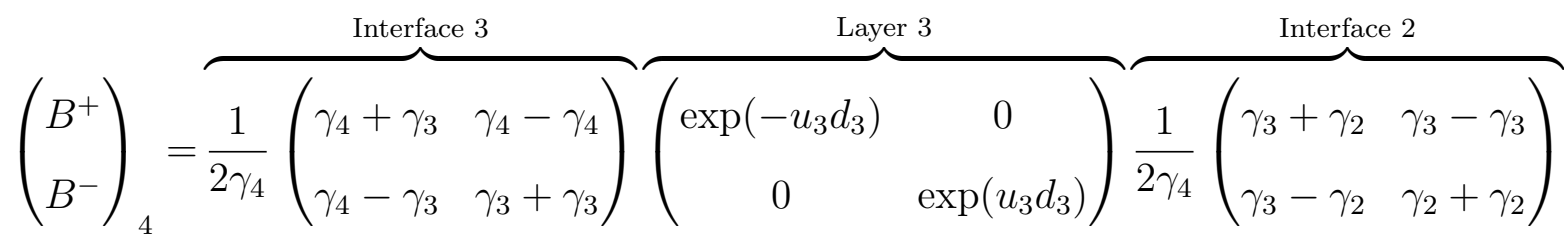

$$
\begin{aligned}
& \underbrace{\left(\begin{array}{cc}
\exp \left(-u_{2} d_{2}\right) & 0 \\
0 & \exp \left(u_{2} d_{2}\right)
\end{array}\right)}_{\text {Layer } 2} \underbrace{\frac{1}{2 \gamma_{5}}\left(\begin{array}{cc}
\gamma_{2}+\gamma_{1} & \gamma_{2}-\gamma_{1} \\
\gamma_{2}-\gamma_{1} & \gamma_{2}+\gamma_{1}
\end{array}\right)}_{\text {Interface } 1} \underbrace{\left(\begin{array}{l}
0 \\
1
\end{array}\right)}_{\text {Boundary }}
\end{aligned}
$$

Contained in Equation 3.7 are the thermophysical properties of each layer within the sample. With $V_{3 \omega}, \frac{d R}{d T}, V_{1 \omega}$ and $R_{0}$ measured, then $T_{2 \omega}$ can be determined through Equation 3.1. With the dimensions of the heating wire known, then the only unknowns remaining in Equation 3.2 are the thermophysical properties contained in either the $A$ and $B$ terms.

This algorithm developed by Feldman in this form does not take into account lateral spreading of heat flux prior to entering the substrate. It generally assumes that the thickness of the films is much less than the width of the heating wire. However lateral spreading can be accounted for if necessary as shown by Borca-Tasuic.[75]. Alternatively, the error caused by lateral spreading can be quantified as discussed by Dames.[88] Specifically, the term $b / d \sqrt{k_{z} / k_{x}}$ should be considered where $d$ is 
the film(s) thickness, $b$ is the heater width and $k_{z} / k_{x}$ is the anisotropic ratio. If $b / d \sqrt{k_{z} / k_{x}}>30$ then the error induced is less than $1 \%$. If $b / d \sqrt{k_{z} / k_{x}}>5.5$ then the error induced is less than 5\%. The thin film aerogel samples discussed in Chapter 4 generally have an error of about $2 \%$ induced by lateral spreading. The relatively thicker and anisotropic carbon nanotubes discussed in Chapter 6 have an error closer to $5 \%$ due to lateral spreading.

\subsubsection{General sensitivity}

One cannot assume every thermophysical property of the system can be simultaneously fit. Ideally, many of these parameters are already known and used as system inputs. To determine if one can reasonably fit one or more thermophysical properties of the system, sensitivity analysis is employed. Consider the normalized sensitivity, $S_{p}$, of the $V_{3 \omega}$ signal when the parameter of interest, $p$, is perturbed by $\Delta$ which is set to $10 \%$ in this work.

$$
S_{p} \omega=\frac{\left(V_{3 \omega}(\Delta)-V_{3 \omega}(0)\right) / V_{3 \omega}(0)}{(\Delta p-p) / p}=\frac{V_{3 \omega}(\Delta)-V_{3 \omega}(0)}{V_{3 \omega} \Delta}
$$

The greater the relative influence of the parameter, the easier it is to isolate its contribution to the output signal. If multiple parameters are to be fit, it is important that each has a unique contribution to $S_{p}$ over at least a portion of the frequency window. Figure 3.6 shows the sensitivity of the experiment to each thermal property for a three material system in which the layers are an amorphous $\mathrm{SiO}_{2}$ substrate, a micron thick layer of silver, and a silicon dioxide capping layer $200 \mathrm{~nm}$ thick to electrically isolate the sample. The thermal conductivity of the substrate and capping layer would likely be independently determined and used as inputs. One could use this scenario to study the changing material properties of this intermediate metallic layer as some condition is altered. If one needed to shift the sensitivity range of each material, this can be done by changing the heater width. In general, more narrow 


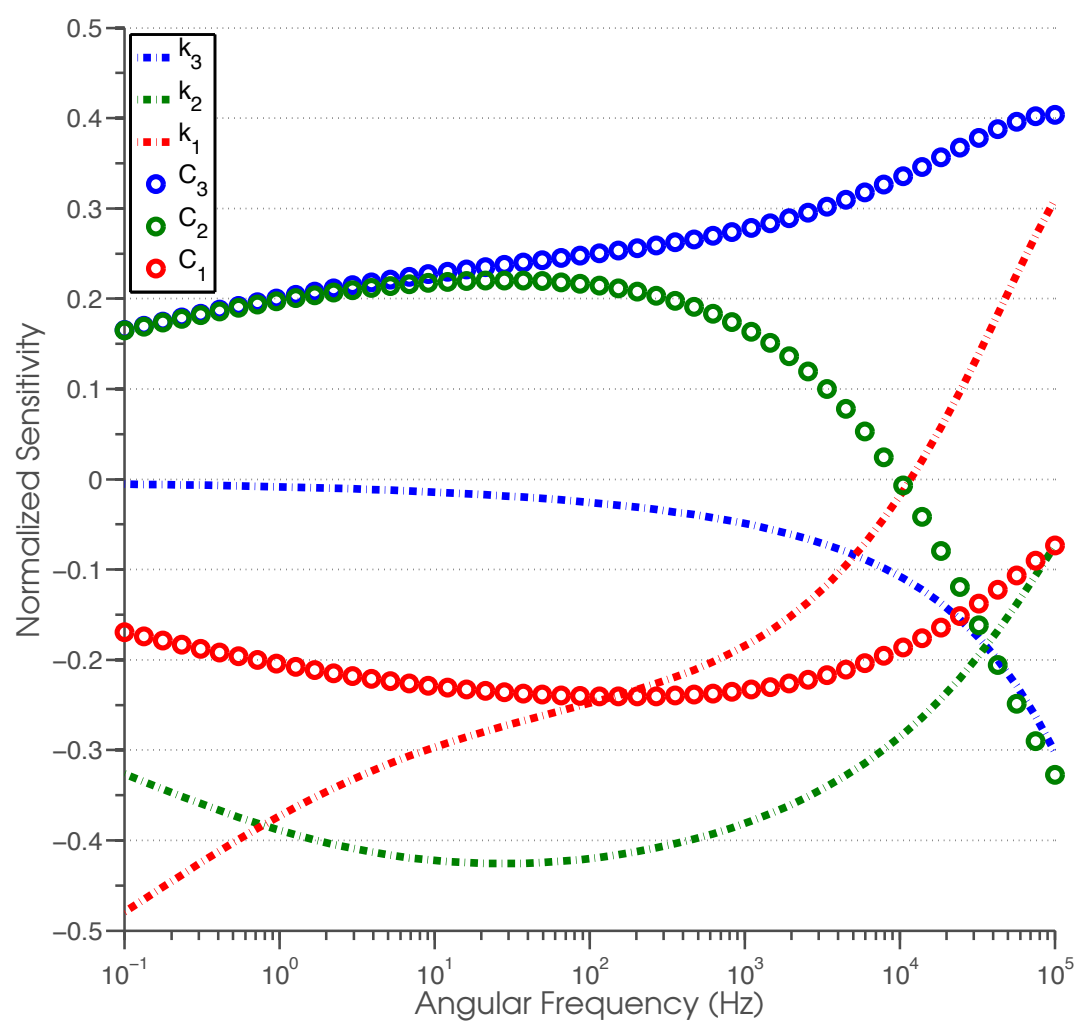

FiguRE 3.6: Sensitivity response for a three material system with layers 1,2 and 3 using the known properties of an amorphous $\mathrm{SiO}_{2}$ substrate, a micron thick silver film and a $200 \mathrm{~nm}$ thick $\mathrm{SiO}_{2}$ film respectively. The input heater width was 30 microns.

heaters have increased sensitivity to properties near the metal line.

The matrix algorithm used can also take into account the boundary resistance at an interface. While the thermal boundary resistance between two atomically bonded layers can influence the total thermal transport within a thin film system, it is not the focus of this study. Figure 3.7 shows the sensitivity of the experiment to a three layer aerogel sample similar to those studied in Chapter 4. A contact resistance of $10^{-7} \mathrm{~m}^{2} \mathrm{k} / \mathrm{W}$ is selected as the input. This is an order of magnitude greater than what others have measured as a contact resistance between $\mathrm{SiO}_{2}$ and a metal to serve as a suitable bound for the most influence this resistance could have on the signal.[89] As shown in Figure 3.7 the influence of the boundary resistances $R_{32}$ and $R_{21}$ are very small over this frequency range, and negligible when compared to the sensitivity of 


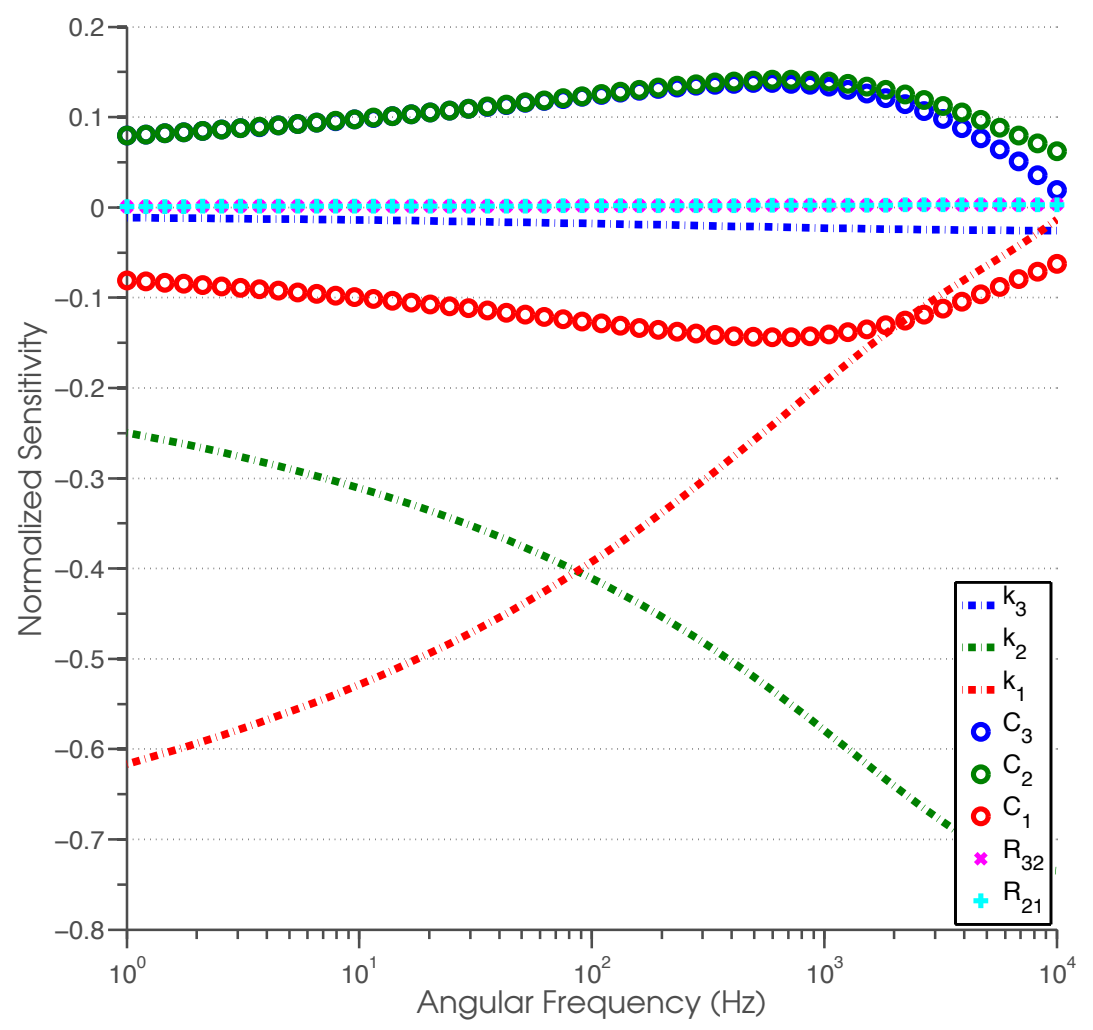

FiguRE 3.7: Sensitivity plot considering thermal boundary resistance for a three layer system. The top and bottom layers are amorphous $\mathrm{SiO}_{2}$. The central layer is a thin film aerogel, similar to those studied in Chapter 4, with an input thermal conductivity of $0.02 \mathrm{~W} / \mathrm{m} \mathrm{K}$ and thickness of $500 \mathrm{~nm}$. The input thermal boundary resistance is $10^{-7} \mathrm{~m}^{2} \mathrm{k} / \mathrm{W}$

other parameters. At frequencies greater than $10^{5} \mathrm{~Hz}$ the two terms start to have a significant influence. Additionally by reducing the width of the heating line, the experiment has increased sensitivity to these boundary resistance terms.

In both the scenario of a silver film depicted in Figure 3.6 and the scenario of an aerogel film depicted in Figure 3.7, the material of interest (Layer 2) has a unique thermal conductivity and volumetric heat capacity response. Over the frequency regimes measured, the experiment is sensitivite to the thermal diffusivity of the material. Thermal diffusivity is related to the rate at which a material matches the temperature of its surroundings. However, frequency domain experiments are not always independently sensitive to a material's thermal conductivity and heat 


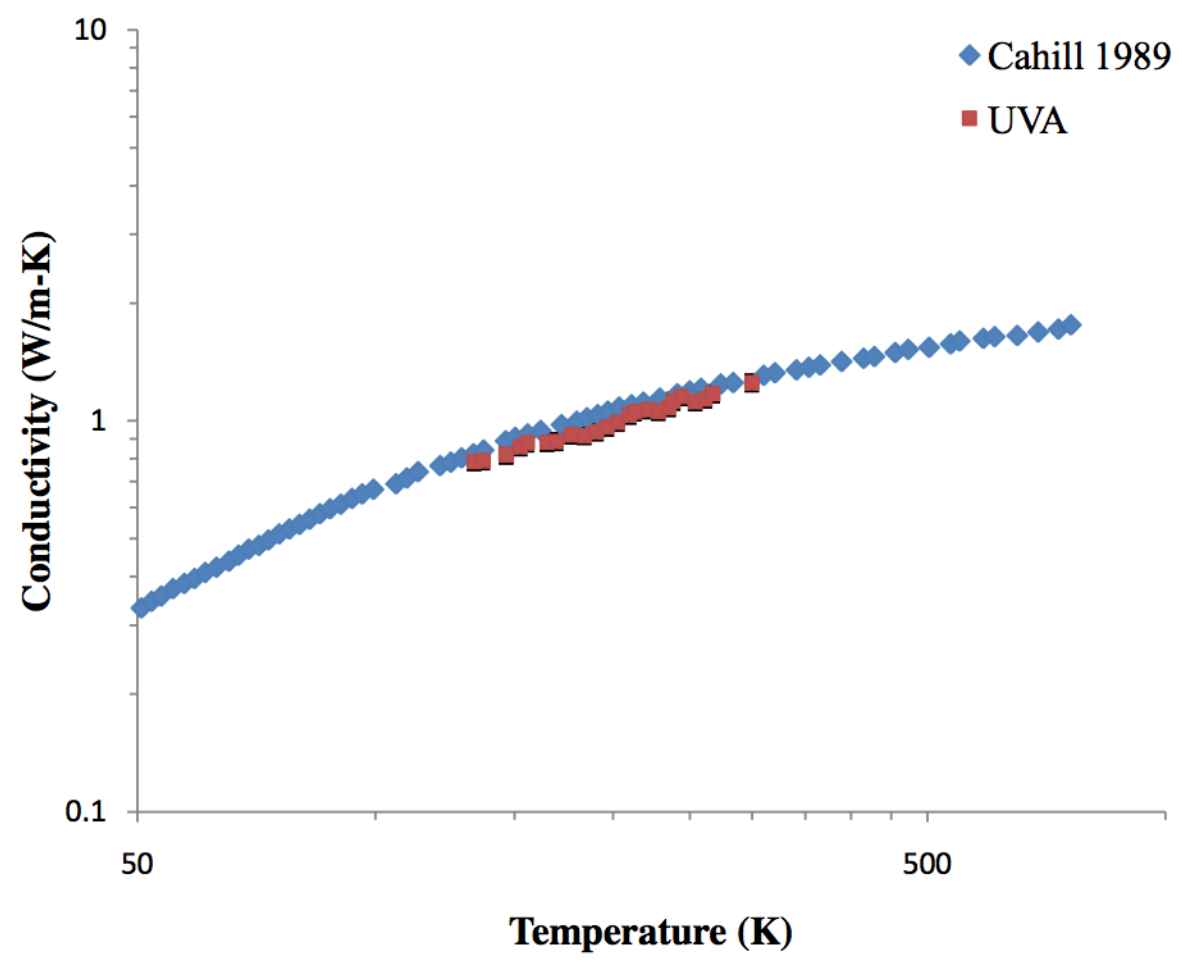

FiguRE 3.8: Measured thermal conductivity of the first sample tested at UVA, an amorphous $\mathrm{SiO}_{2}$ substrate, compared with data from previous research.[1]

capacity. In specific scenarios the temperature rise may be related to the material's thermal effusivity, which is related to the rate at which a material exchanges heat with the environment. Thermal effusivity is a function of the product of a material's heat capacity and thermal conductivity. When an experiment is only sensitive to the thermal effusivity of a material, one cannot differentiate between the effects of its thermal conductivity and heat capacity. This scenario arises in the bidirectional apparatus presented in the subsequent section.

\subsubsection{Initial $\mathrm{SiO}_{2}$ test}

Figure 3.8 shows the thermal conductivity of an amorphous $\mathrm{SiO}_{2}$ substrate measured from $77 \mathrm{~K}$ to room temperature. A niobium heating wire was adhered to the sample with a thickness of $200 \mathrm{~nm}$, a width of 10 microns, and a length of 7 $\mathrm{mm}$. The thermoresistive response of the wire was $0.47 \Omega / \mathrm{K}$. The similarity 
between our results and another study on the same material helps confirm the successful construction of the experiment, micro fabrication on the sample, and analysis of data.

\subsection{Development of a bidirectional $3 \omega$ system}

This section discusses the development of a unique $3 \omega$ mounting scheme. Much of the content in this section and figures used are reproduced from our publication "General bidirectional thermal characterization via $3 \omega " .[4]$

\subsubsection{Motivation}

Discussion of $3 \omega$ to characterize solid thin films up to this point has assumed that one can deposit a thin metal heating line and contact pads directly on the sample. However the prerequisite deposition, photolithography, and etching of the metal segment on the sample of interest can be difficult or even impossible on some unique samples. This problem is highlighted in two phase systems in which voids are a large portion or majority of the sample. In some situations, such as the aerogel films studied in Chapter 4, a protective capping layer is sufficient to create a planer surface onto which the metal layer can be adhered. However, in other situations, this is not possible. Using electron beam evaporation, I was not successful in depositing a continuous layer on the free end of vertically aligned carbon nanotube arrays (studied in Chapter 6). Additionally, for studies in which pressure is to be applied to a sample resulting in straining, it is unlikely the metal wire could maintain its well defined geometry while under pressure (such as was necessary for the carbon fiber study in Chapter 5).

To overcome this challenge, a bidirectional testing apparatus was developed in which the heating wire is adhered to a separate mount and the sample is pressed to the metal line. This creates the additional advantage of allowing for reuse of the 
metal test line for large series of samples, avoiding the need to repeat the deposition and lithography process for each sample individually. Furthermore, samples with properties dependent on the applied pressure can be characterized under varying loads in such an apparatus without altering the linear uniformity of the heating wire which generates the geometrically defined heating event. This approach has a few drawbacks that must be considered. The contact resistance between the sample and heating source is an additional fitting parameter. It is not possible to determine this parameter simultaneously with the sample's thermal conductivity in all situations, but it will be shown that in many scenarios the experiment can be adapted to overcome this obstacle. The thermophysical properties of the layers beneath the heating wire, as well as the potential passivation layer between the mount and sample are additional inputs that must be well defined or else they will be detrimental to achieving quality fits for collected data. Lastly, an accurate thermal model of the system must be considered to account for the experiment's increased complexity. This concept builds on bidirectional $3 \omega$ systems of other researchers $[90,91]$ and aims to provide a general layout of such a system with an intuitive solution to the heat diffusion equation, avoiding any limiting assumptions.

\subsubsection{Mounting scheme fabrication}

The mount onto which the sample is to be pressed must meet several design criteria. It must be able to be placed into a cryostat so that the samples can be measured under vacuum pressure and undergo at least moderate temperature ramping. It should reach the equilibrium temperature of the cryostat reasonably quickly. The top layer must be planar so that continuous, geometrically defined metal lines can be deposited on it. Additionally this layer must be electrically insulating to keep the signal isolated. Ideally this layer should also be thermally resistive to maximize the percentage of heat flowing into the sample (rather than into the mount) and therefore maximize the signal to noise ratio of the experiment 
(a)

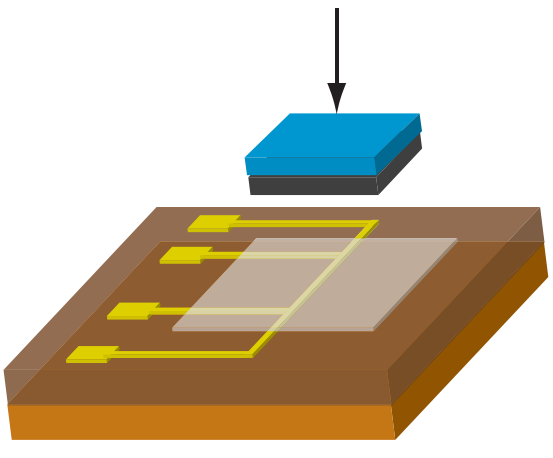

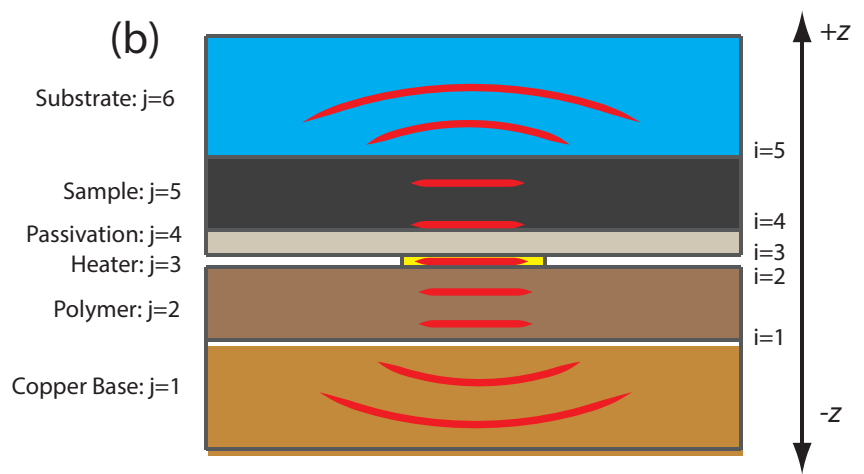

Figure 3.9: The left image depicts the $3 \omega$ mount with a sample pressed to it. The lowest layer of the mount is a copper slab which attaches to the cryostat. Fabricated onto it from bottom to top is the SU-8 polymer layer, the gold $3 \omega$ pads and heating line, and an $\mathrm{SiO}_{2}$ passivation layer. The pads are connected to the remainder of the circuit via silver paste. The sample is pressed onto the central portion of the metal line by an external force. The right image represents these six layers (with index $j$ ) with the apparent stratified medium perspective of the system. Interfaces, labeled $i$, separate each layer. Thermal waves propagate from the central heating layer $(j=3)$ in both the $+z$ direction toward passivation layer, sample, and substrate $(j=4,5,6)$, and the $-z$ direction into SU-8 photoresist and copper base $(j=2,1)$

by creating larger $T_{2 \omega}$. The mounting system must be able to withstand a moderate amount of pressure. Lastly, an electrically isolating layer must be deposited above the heating wire if electrically conductive samples are tested.

After discussions with Professor Lichtenberger, the concept depicted in Figure $3.9 \mathrm{a}$ was developed. Thermal energy flows from the heating wire to both the mount it is adhered to and the sample of interest. The layers surrounding the heating line are electrically insulating. A heating wire is deposited on a polymer layer to achieve the requisite design criteria. Polymers generally have a thermal conductivity on the order of $0.2 \mathrm{~W} / \mathrm{m} \mathrm{K}$, an order of magnitude lower than other common amorphous materials. A $6.4 \mathrm{~mm}$ thick copper sheet, machined to fit the cryostat base, is used as the lowest layer of the mount. SU-8 3025 series photoresist made by MicroChem is then spin coated onto the copper base at $1500 \mathrm{rpm}$. This rotation speed is selected to maximize the thickness while still obtaining a planar surface. Spin speeds were attempted ranging from 500 to $5000 \mathrm{rpm}$ before settling on this speed. The copper and photoresist are soft baked at $95^{\circ} \mathrm{C}$ for an hour on a hot plate, cooled to room temperature, then slowly hard baked on a hot plate, 
increasing the temperature by $20^{\circ} \mathrm{C}$ every 30 minutes. The mount is held at a maximum temperature of $200^{\circ} \mathrm{C}$ for two hours before being allowed to cool by stepping down the hotplate temperature. Faster ramping processes caused cracking in the surface of the polymer which became apparent when observing the metal line under a microscope (see Figure 3.10). The thickness of the SU-8 is determined by white light interferometry. This value varied from $35-90 \mu \mathrm{m}$ on different mounts. Thickness measurements were taken at several locations to ensure uniformity across the mount, particularly in the region near the central heating portion of the wire. This process did not have a 100\% success rate. Generally 5 mounts would be made in parallel and 3 would ultimately be good candidates for implementing. Slower heat treatment, and a faster spin speed causing greater uniformity of the polymer across the copper base would have likely generated a higher success rate.

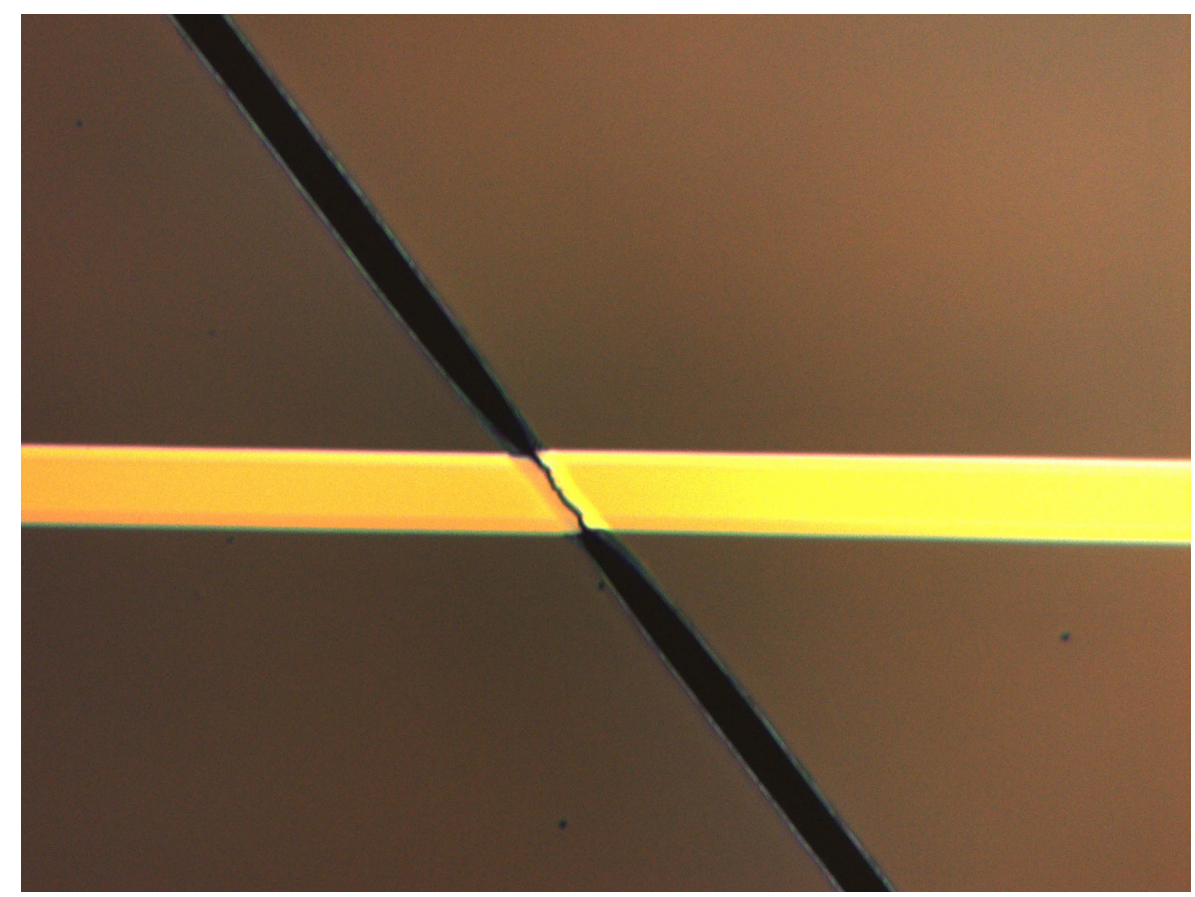

FIgURE 3.10: Crack in the polymer identified after the deposition and etching of the gold metal line.

Next a metal layer was deposited onto the mount. A $200 \mathrm{~nm}$ Au film with a $5 \mathrm{~nm}$ Ti layer for adhesion was deposited using electron beam lithography. The metal was 
selected under the belief that the mount would be electrically connected to the rest of the circuit via gold wire bonding. Silver paste produced by Structure Probe Inc. was ultimately selected to connect the pads on the mount to the rest of the circuit, however the microfabrication process utilizing a gold heating wire remained.

Initially a wet etch liftoff photolithography process was attempted to pattern the $3 \omega$ mask onto the mount. AZ-nLof 2020 was used as a negative photoresist to form the pattern of the metal pads and lines. To remove the unwanted portion of the gold layer, the mount was submerged in a 1:1 mix of N-methyl-2-pyrrolidone (NMP) and propylene glycol with a rotating stir bar heated to $120^{\circ} \mathrm{C}$ for 60 minutes. Ethylene glycol was then applied to the surface after the mount was cooled to remove the unwanted gold. However, the process weakened the bonds on the surface of the SU-8. The critical portion of the heating wires would come off easily (while not as easily as the other potions). Shorter soak times were attempted, but an ideal process was not determined where the excess gold could be removed without harming portions of the gold intended to remain permanently on the mount.

Instead, successful etching of the gold was performed using a physical bombardment of argon atoms in the Axic reactive-ion etching tool in the University of Virginia's Microfabrication Laboratory (UVML). The RF source was set to 200 $\mathrm{W}$ and the chamber pressure at $20 \mathrm{mT}$. Etching occurred in 10 minute increments to prevent the sample mount from reaching elevated temperatures. Generally 3-4 10 minute etches were sufficient to remove the excess gold. The sample was then placed in an $\mathrm{O}_{2}$ etch overnight operating at $100 \mathrm{~W}$ to remove the protective photoresist.

$\mathrm{SiO}_{x}$ was sputtered onto the mount to form the top most layer, the passivation layer. This layer was then etched using the Oxford reactive ion etching tool in UVML. $\mathrm{CHF}_{3}$ flowed into the etching chamber at $50 \mathrm{sccm}$ with an RF power of $80 \mathrm{~W}$ and a pressure of 10 mTorr for 4 minutes. After etching and photoresist removal, $\mathrm{SiO}_{x}$ remained above the central heating portion of the wire, providing electrical isolation between the line and the sample. Initially this layer was deposited to be $20 \mathrm{~nm}$ 
thick. After several tests it was found that with sufficient pressure, samples such as carbon nanotube arrays would puncture through the layer after repeated tests, making electrical contact with the wire. Later mount versions used a nominally 200 nm thick passivation layer. As discussed later, this increased thickness did not have a significant negative effect on the system's sensitivity to the parameters of interest.

\subsubsection{Experiment operation}

After completing fabrication, the mount could then be implemented into the $3 \omega$ circuit. The mount was screwed onto the cryostat base. The electrical pads were connected to the circuit using silver paste, which was given 16 hours to dry before use. Prior to testing unique material systems, the thermophysical properties of the mounting system needed to be determined. Specifically, it was necessary to determine the thermal conductivity and volumetric heat capacity of the SU-8 layer and $\mathrm{SiO}_{2}$ passivation layer. To simplify fitting, a mount system was tested without the presence of the passivation layer, leaving only the gold wire, polymer, and copper substrate. This scenario is the typical film on substrate configuration for which $3 \omega$ has been utilized for decades. The spatial dimensions of the heating wire are known: $10 \mathrm{~mm}$ long, 37-40 $\mu \mathrm{m}$ wide (dependent on photolithography alignment) and $200 \mathrm{~nm}$ thick. The thermophysical properties of the copper mount are assumed to be bulk values. The SU-8 thickness is known (ranging from 35-90 microns on different mounts). The thermoresistive response of the heating heating wire was determined by temperature ramping from $300 \mathrm{~K}$ to $350 \mathrm{~K}$ in $10 \mathrm{~K}$ increments (typical values were near $0.1 \Omega / \mathrm{K}$ and highly repeatable).

The only remaining parameters to be fit are the thermal conductivity and volumetric heat capacity of the hard baked photoresist. The best fit results varied slightly between mounts. This could be in part due to minor variations in the heat treatment processes, or aging of the photoresist prior to use. Across all mounts measured hard baked SU-8 3025 thermal conductivity was between $0.142 \mathrm{~W} / \mathrm{m} \mathrm{K}$ 
and $0.174 \mathrm{~W} / \mathrm{m} \mathrm{K}$. The thermophysical properties of the $\mathrm{SiO}_{2}$ passivation layer were difficult to fit as part of the larger mount due to a small sensitivity to the layer. Instead, the oxide was always sputtered on a separate substrate simultaneously as each mount was fabricated. This seperate substrate was then used to verify the thickness of the layer and to independently verify the layer's thermal conductivity by fabricating a set of $3 \omega$ pads directly onto the $\mathrm{SiO}_{2}$. Thermophysical properties of the layer varied between different depositions. This could be a function of the purity of the sputter target, chamber cleanliness, or due to changing RF sources. The thermal conductivity of the $\mathrm{SiO}_{2}$ layer varied from a minimum tested value of 0.7 $\mathrm{W} / \mathrm{m} \mathrm{K}$ to a maximum tested value of $1.1 \mathrm{~W} / \mathrm{m} \mathrm{K}$. The appropriate value was recorded with each mount and used as an input.

With the thermophysical properties of the mount determined, a sample could be placed top-side facing down above the central heating wire. Pressure was applied to the sample by placing a plate above it, and increasing the torque on two screws connecting the plate to the mount base and cryostat with the sample sandwiched between the layers. Using a torque wrench, and knowing the properties of the screw as well as the friction coefficient between the screw and copper mount, the torque applied to the screws could be converted into an applied pressure. Early mounts made out of an amorphous $\mathrm{SiO}_{2}$ substrate often cracked with around 100-200 kPa of pressure applied. Maximizing pressure is generally ideal for minimizing the contact resistance between the sample and mount and maximizing the sensitivity to the material's thermal conductivity.

The relationship between the torque required to overcome friction and lower the load for a single screw is $[92]$

$$
T=\frac{F d_{m}}{2}\left(\frac{\pi f d_{m}-l}{\pi d_{m}+f l}\right)
$$

where $F$ is the applied force, $d_{m}$ is the mean screw diameter, $f$ is the static friction 
coefficient, and $l$ is the height the screw moves from one full rotation, the screw pitch. Knowing the properties of the screws, the area of contact between the plate and the sample, and using a torque wrench when loading the sample, the force applied (and therefore pressure applied) can be determined. For the mounting system used the assumed friction coefficient was 0.2 , the mean screw diameter was 0.1225 inches and the pitch was 0.025 inches. Typical measured torques were on the order of 0.5 to 12 inch-oz.

\subsubsection{Determining thermophysical properties}

The thermal model of the system is depicted in figure Figure 3.9b. Below the heating wire (layer 3 ) are the polymer and copper base, layers 2 and 1 respectively. Above the heating wire are the passivation layer, the sample of interest, and in some scenarios a sample substrate, layers 4,5 , and 6 respectively. The contact resistance between layers 3 and $4(i=3)$ must be considered. It can be assumed that both the top layer and bottom layer are semi-infinite. Considering the $\widetilde{A}$ components of the temperature vector traveling in the positive $z$ direction into the sample and the $\widetilde{B}$ components traveling in the negative $z$ direction into the mount, the following boundary conditions are imposed: $A_{z=\infty}^{+}=1, A_{z=\infty}^{-}=0, B_{z=-\infty}^{+}=0$, and $B_{z=-\infty}^{-}=1$. By successively applying Equations 3.5 and 3.6 the components of the temperature rise at the heater can be determined: 


$$
\begin{aligned}
& \left(\begin{array}{c}
A^{+} \\
A^{-}
\end{array}\right)_{3}=\overbrace{\frac{1}{2 \gamma_{3}}\left(\begin{array}{cc}
\gamma_{3}+\gamma_{4} & \gamma_{3}-\gamma_{4} \\
\gamma_{3}-\gamma_{4} & \gamma_{3}+\gamma_{4}
\end{array}\right)}^{\text {Interface 3 }} \overbrace{\left(\begin{array}{cc}
\exp \left(u_{4} d_{4}\right) & 0 \\
0 & \exp \left(-u_{4} d_{4}\right)
\end{array}\right)}^{\text {Layer 4 }} \\
& \text { Interface } 4
\end{aligned}
$$

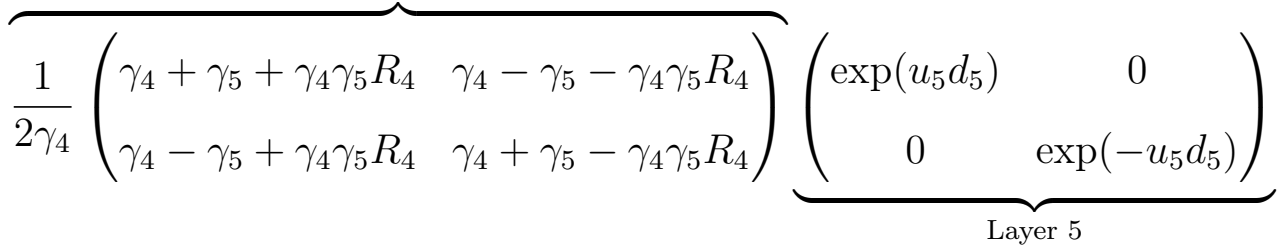

$$
\begin{aligned}
& \underbrace{\frac{1}{2 \gamma_{5}}\left(\begin{array}{cc}
\gamma_{5}+\gamma_{6} & \gamma_{5}-\gamma_{6} \\
\gamma_{5}-\gamma_{6} & \gamma_{5}+\gamma_{6}
\end{array}\right)}_{\text {Interface } 5} \underbrace{\left(\begin{array}{l}
1 \\
0
\end{array}\right)}_{\text {Boundary }} \\
& \left(\begin{array}{l}
B^{+} \\
B^{-}
\end{array}\right)_{3}=\underbrace{\frac{1}{2 \gamma_{3}}\left(\begin{array}{cc}
\gamma_{3}+\gamma_{2} & \gamma_{3}-\gamma_{2} \\
\gamma_{3}-\gamma_{2} & \gamma_{3}+\gamma_{2}
\end{array}\right)}_{\text {Interface } 2} \underbrace{\left(\begin{array}{cc}
\exp \left(-u_{2} d_{2}\right) & 0 \\
0 & \exp \left(u_{2} d_{2}\right)
\end{array}\right)}_{\text {Layer } 2} \\
& \underbrace{\frac{1}{2 \gamma_{2}}\left(\begin{array}{cc}
\gamma_{2}+\gamma_{1} & \gamma_{2}-\gamma_{1} \\
\gamma_{2}-\gamma_{1} & \gamma_{2}+\gamma_{1}
\end{array}\right)}_{\text {Interface } 1} \underbrace{\left(\begin{array}{l}
0 \\
1
\end{array}\right)}_{\text {Boundary }}
\end{aligned}
$$

We are concerned with fitting the sample thermal conductivity, $k_{5}$, the sample volumetric heat capacity, $C_{5}$, and the contact resistance between the sample and the passivation layer, $R_{4}$ Each remaining thermophyscial property is independently verified as previously discussed.

\subsubsection{Parameterized sensitivity analysis}

The complexity of the system warrants an exploration of the scenarios under which the variables of interest are discernible. Figure 3.11 is generated to consider both the parameters of interest and the heater width's effect on the sensitivity of the system. Equation 3.8 is evaluated for the three fitting variables: $k_{5}, C_{5}$, and $R_{4}$. At 

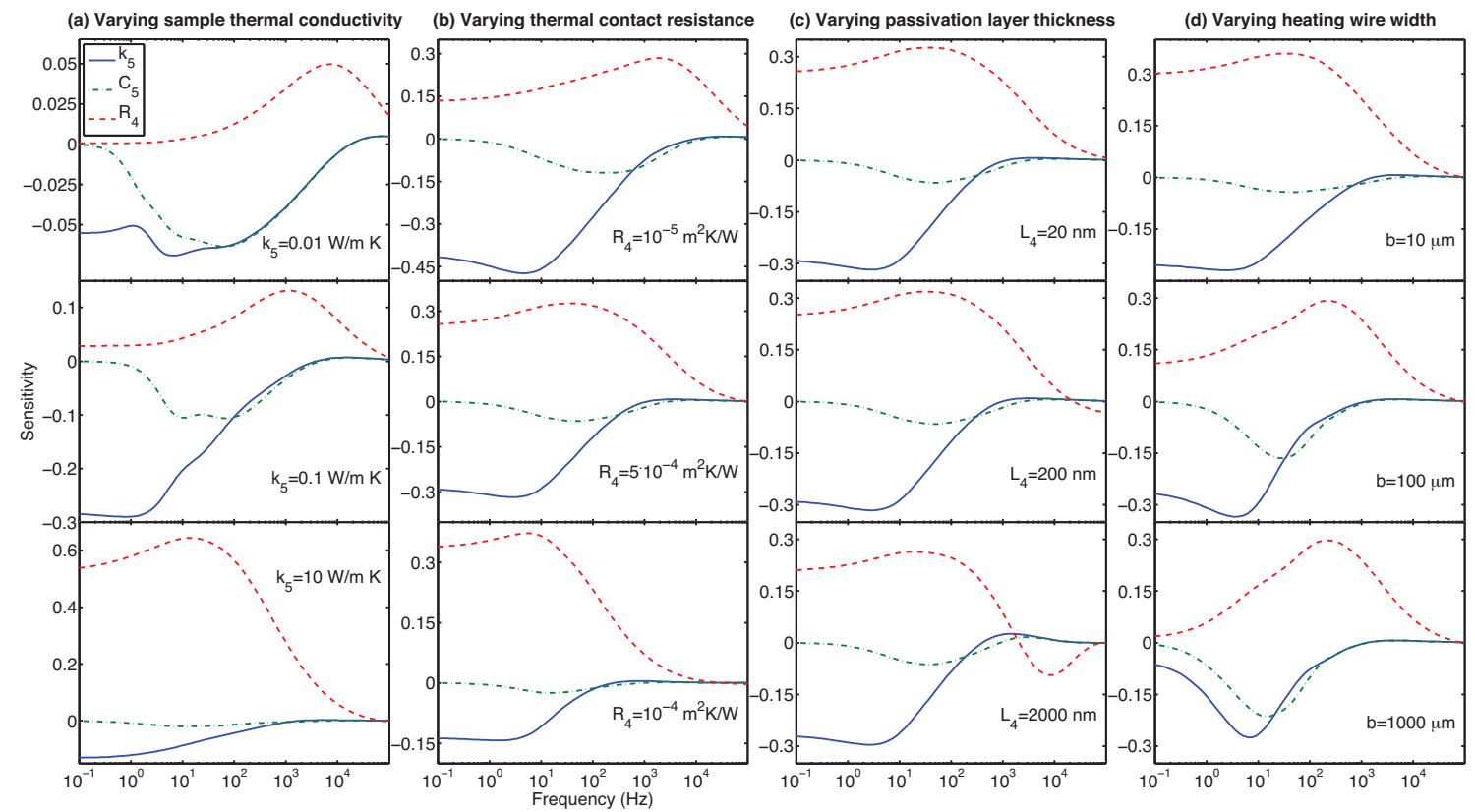

FiguRE 3.11: Plots of fitting parameter sensitivity trends over a wide frequency range in the six layer system. In all scenarios the sample of interest is set to a thickness of $90 \mu \mathrm{m}$ and a volumetric heat capacity of $1 \mathrm{MJ} / \mathrm{m}^{3} \mathrm{~K}$ on an amorphous $\mathrm{SiO}_{2}$ substrate at room temperature. The passivation layer, $\mathrm{SiO}_{2}$, has an input thermal conductivity of $0.7 \mathrm{~W} / \mathrm{mK}$ and a thickness of $50 \mathrm{~nm}$ unless otherwise stated. The sample's thermal conductivity is $1 \mathrm{~W} / \mathrm{m} \mathrm{K}$ in subfigures (b), (c), and (d). The contact resistance is set to $3 \times 10^{-5} \mathrm{~m}^{2} \mathrm{~K} / \mathrm{W}$ in subfigures (a), (c), and (d). The copper mount layer is assumed to have bulk properties. 
lower frequencies the system responds identically to a dc source term, causing each sensitivity variable to remain constant. At higher frequencies, each term approaches zero and noise in the measurement becomes dominant.

Ideally the experiment is sensitive to each fitting parameter in a unique frequency regime. If this scenario does not exist, a unique trend must be present for each fitting parameter. In Figure 3.11(a), as $k_{5}$ is increased the contact resistance sensitivity range is shifted to lower frequencies, overlapping with the sensitivity window of $k_{5}$ and $C_{5}$ and increasing in relative magnitude, making fitting the two sample properties difficult. At mid to high frequencies, when the experiment becomes effectively one dimensional, the sample's thermal conductivity and heat capacity can become indistinguishable and only the material's thermal effusivity $(\epsilon=\sqrt{k C})$ is discernible as can be seen in the top plot Figure 3.11(a). Figure 3.11(b) illustrates that by decreasing the contact resistance, operators have a means to shift the peak response of $S_{R_{4}}$ to higher frequency ranges providing a cleaner simultaneous fit of the three parameters. In many scenarios, when an insufficient pressure is applied, the contact resistance term dominates the output in the high frequency range. The sample's volumetric heat capacity sensitivity trend, $S_{C_{5}}$, is nearly always most unique in a middle frequency range, and the sample's thermal conductivity sensitivity trend, $S_{k_{5}}$, is generally most unique at a low frequency range near $1 \mathrm{~Hz}$.

The effects of each fitting variable on a frequency sweep are most easily identified when each sensitivity term, $S_{p}$, occupies a unique frequency regime. However, applying a large load onto a conductive sample can cause a breach in the passivation layer. Figure 3.11(c) shows that increasing the thickness of this layer to prevent the sample from penetrating the passivation layer is not necessarily detrimental to the critical sensitivity terms. Furthermore, varying this layer's thickness, $L_{4}$, provides another means of tuning the unique trend of $S_{R_{4}}$ away from that of $S_{K_{5}}$.

Of equal importance to ensuring that a unique sensitivity trend exists for each 
parameter, is ensuring the magnitude of each sensitivity parameter is sufficiently large. This is the second problem illustrated in the bottom plots of Figure 3.11(a) and (b). The sample's volumetric heat capacity sensitivity, $S_{C_{5}}$, is more than an order of magnitude smaller than the contact resistance sensitivity, $S_{R_{4}}$, at all frequencies. To aid in maximizing sensitivity to $k_{5}$ and $C_{5}$, one should minimize the combined thermal impedance of the passivation layer and the contact resistance. For example, if a sufficiently small contact resistance cannot be achieved because the sample punctures the passivation layer and interacts electrically with the wire, the passivation layer thickness should be increased to minimize the total impedance of layer 4 and interface 4 over the critical frequency range. Additionally if these steps are insufficient, broadening the heating wire width can further alter the sensitivity window to each parameter, as shown in Figure 3.11(d) and discussed further by Raudzis et al.[93]

As with all thermal experiments, highly conductive thin samples are the most challenging to fit due to a small peak sensitivity to the sample's thermal conductivity. However this experiment's strength lies in characterizing compliant, often two-phase, systems in which the thermal conductivity is relatively small. Carbon nanotube arrays, a notable exception to this statement, have a superior sensitivity compared to an isotropic counterpart with the same thermal conductivity and are a reasonable material to characterize with this experimental apparatus, [90] particularly if lower frequencies are utilized in data fitting. Generally, the sample's heat capacity has the smallest peak sensitivity and the greatest uncertainty associated with it of the three fitting variables.

\subsubsection{Sample measurement}

To demonstrate the feasibility of the experimental setup, a $1.5 \mathrm{~mm}$ thick cut of

natural gum rubber was investigated at several unique loading pressures. The SU-8's thickness was separately measured to be $63 \mu \mathrm{m}$. A $240 \mathrm{~nm} \mathrm{SiO}_{2}$ passivation 


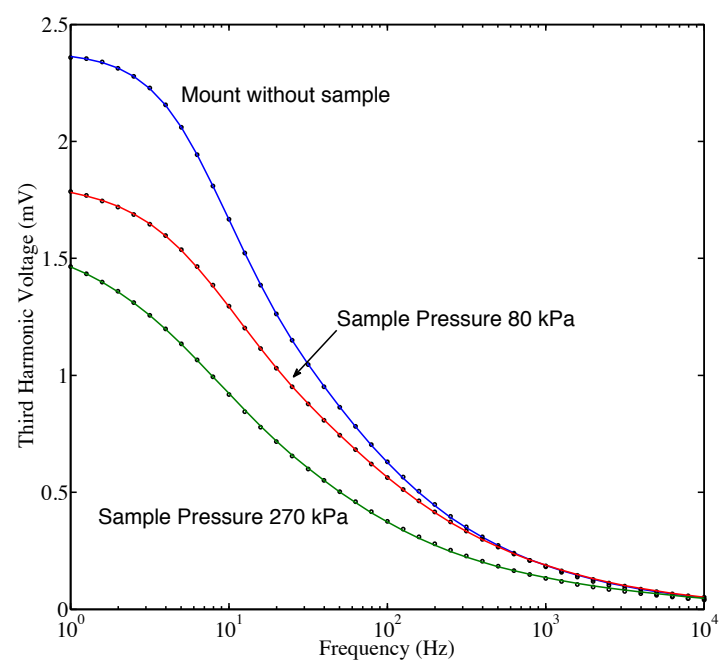

FiguRE 3.12: In-phase $V_{3 \omega}$ data collected and best fit results of the experiments for three scenarios: the mount under vacuum with no sample, and the sample present at two unique pressures. All data was collected at $297 \mathrm{~K}$.

layer is present on the mount, although not required for this sample because it is electrically insulating. The $\mathrm{SiO}_{2}$ layer was characterized on a separate sample (deposited simultaneously with mount's layer) in a traditional $3 \omega$ film on substrate setting. Its thermal conductivity was determined to be $0.7 \mathrm{~W} / \mathrm{m} \mathrm{K}$. The thermoresistive response of the wire was $0.110 \Omega / K$ obtained from 11 measurements between $300 \mathrm{~K}$ and $350 \mathrm{~K}$ resulting in an uncertainty of $0.4 \%$. The best fit thermal conductivity and volumetric heat capacity of the hard baked SU-8 3025 series, 0.14 $\mathrm{W} / \mathrm{m} \mathrm{K}$ and $2.1 \mathrm{~W} / \mathrm{m}^{3} \mathrm{~K}$ respectively, are obtained by performing a frequency sweep on the mount in vacuum with no sample present.

Data fits for the isolated mount, and the system with a sample present are shown Figure 3.12. As the figure implies, with greater sample pressure the contact resistance is reduced, allowing thermal energy to more rapidly dissipate from the heating wire. This decreases the temperature rise of the wire, $T_{2 \omega}$. In attempted fittings with incorrect inputs (such as assuming the thermal conductivity of the passivation matched bulk amorphous $\mathrm{SiO}_{2}$ ), the best data fits were observed to clearly follow an incorrect trend in at least one frequency regime. 
The results obtained from each scan are displayed in Table 3.1. Over this relatively low pressure range, there is nearly no change in the sample's thermal conductivity nor heat capacity. The contact resistance exponentially decreases as increasing pressure is applied to the sample, asymptotically approaching a minimum value near zero. The best fit thermal conductivity and volumetric heat capacity are in agreement with accepted values for soft vulcanized rubber, which has been reported as $0.13 \mathrm{~W} / \mathrm{mK}$ and $2.2 \mathrm{MJ} / \mathrm{m}^{3} \mathrm{~K}$ respectively.[94, 95] The pressure ranges tested did not significantly strain the sample, causing only minor changes in density. The data collected at 50 $\mathrm{kPa}$ pressure did not give a sensical heat capacity result. Sensitivity analysis revealed that at contact resistances somewhat greater than $10^{-4} \mathrm{~m}^{2} \mathrm{~K} / \mathrm{W}$, sensitivity to the sample's heat capacity was dwarfed by a similar shaped sensitivity to the thermal contact resistance, similar to the middle plot in Figure 3.11b. It is estimated that the contact resistance needs to be less than $1.5 \times 10^{-4} \mathrm{~m}^{2} \mathrm{~K} / \mathrm{W}$ to provide reasonable fits for this sample and mount scenario.

TABLE 3.1: Best Fit Results

\begin{tabular}{lccc}
\hline $\begin{array}{l}\text { Pressure } \\
(\mathrm{kPa})\end{array}$ & $\begin{array}{c}\text { Conductivity } \\
(\mathrm{W} / \mathrm{m} \mathrm{K})\end{array}$ & $\begin{array}{c}\text { Heat Capacity } \\
\left(\mathrm{MJ} / \mathrm{m}^{3} \mathrm{~K}\right)\end{array}$ & $\begin{array}{c}\text { Contact Resistance } \\
\left(10^{-5} \mathrm{~W} / \mathrm{m}^{2} \mathrm{~K}\right)\end{array}$ \\
\hline 50 & 0.11 & - & 20.1 \\
80 & 0.11 & 2.1 & 4.9 \\
110 & 0.11 & 2.2 & 3.4 \\
160 & 0.11 & 2.2 & 2.3 \\
210 & 0.12 & 2.2 & 1.8 \\
270 & 0.12 & 2.2 & 1.4 \\
\hline
\end{tabular}

\subsubsection{Sources of error}

Experimental error can be caused by either inaccuracy in the output signal or incorrect system inputs. It is apparent that accuracy in the $V_{3 \omega}$ signal is critical to the experiment's success. Experience operating the system has shown the higher harmonic voltage measured by a lock-in amplifier is highly repeatable, fluctuating by less than $1 \%$ between frequency sweeps on identical systems. As previously stated, 
we use the common $V_{1 \omega}$ subtraction method to remove what would otherwise be the greatest contributor to random noise in the signal.

Incorrect system inputs can easily be the most significant source of error. In this system the input list is cumbersome: the spatial dimensions and thermoresistive response of the metal line, the thermophysical properties of each layer, and thickness of each layer that cannot be assumed semi infinite. The accuracy to which each of these terms is known drives the uncertainty in our measurements. Wei and coauthors suggest accounting for uncertainty by considering the ratio of the sensitivity of the fit parameters to the sensitivity of each input.[96] This ratio was evaluated for every combination of fitting parameter and input, $S_{f} / S_{i}$, for our system. The selection of a single frequency point to evaluate this ratio can be somewhat arbitrary. However, it is clear that the spatial dimensions of the heating wire, its thermoresitive response, and the thermophysical properties of the layer beneath the metal line $(j=2)$ have the greatest relative sensitivity and therefore influence the uncertainty of the measurement the most. Ultimately, our thermal conductivity and volumetric heat capacity fits of the gum rubber sample have an estimated uncertainty of $9 \%$ and $14 \%$ respectively.

\subsubsection{Summary}

The $3 \omega$ technique is capable of being modified to measure material properties in a bidirectional experimental layout. A stratified medium solution to the thermal diffusion equation accurately describes this scenario. While this format has many advantages, care must be taken to design the experiment to achieve sensitivity to the desired material property. By controlling the pressure applied to the sample and the properties of the passivation layer, a simultaneous fit of the sample properties and the thermal contact resistance is often possible. Using accurate inputs, minimizing measurement error, and minimizing contact resistance are particularly important steps on samples with a large thermal conductivity. A gum rubber 
sample measured using this method was shown to have a minimal change in thermal conductivity or volumetric heat capacity at pressures between $80 \mathrm{kPa}$ and $270 \mathrm{kPa}$. The measured thermophysical properties agree with expected values. These tests give confidence to using the tool for studies in Chapters 5 and 6 . 


\section{Chapter 4}

\section{Thermal Isolation via Thin-Film}

\section{Silica Aerogels}

\subsection{Background and introduction}

The need to minimize thermal transport in micro devices has led researchers to explore material systems of increasing complexity. Due to their unique microstructure, aerogels demonstrate a wide array of structural attributes not found in other two-phase systems which has kept them at the forefront of materials research for decades.[97, 98] First created in 1931 by Steven Kistler,[99] bulk aerogel monoliths have been reported to have the lowest dielectric constant, lowest refractive index, highest surface area, lowest density, lowest acoustic velocity, and lowest thermal conductivity of any known solid.[2] This combination of attributions has led to a wide range of aerogel uses from IR detectors,[100] chemical sensors,[101] and super capacitors,[102] to capturing high velocity space dust particles from speeding comets.[103]

Aerogels are a unique two-phase system containing an open-cell microporous network. Their ultra-low density further distinguishes aerogels from other porous systems, allowing for the extraordinary array of physical attributes. They have been 
created with less than $1 \%$ solid phase composition while maintaining a continuous structure.[97] The extreme thermophysical properties demonstrated by aerogels stem from the fractal nature of the material system as well as discrete structural features on three specific length scales (often labeled micro, meso, and macro). Their structure is highly tunable which makes them excellent candidates for thermal study.

Aerogel properties can vary greatly based on the backbone material and fabrication conditions. The span of achievable properties and the range of materials used to make aerogels has broadened dramatically in the last two decades. Most commonly, aerogels are made of silica, but vanadia,[104] alumina,[105] carbon nanotubes,[106] chalcogenides,[107] iron,[108] carbon,[109] and other metal oxides[110] have also been successfully utilized. The materials used to make aerogels yield properties unique to each aerogel system. Silica aerogels, for example, have yielded some of the lowest thermal conductivities measured in a solid, as low as $0.0006 \mathrm{~W} / \mathrm{mK}[111]$ while graphene aerogels have been shown capable of maintaing a higher electrical conductivity than other porous carbon materials as the density of the material is reduced.[112]

Thin film aerogels are a relatively new subset of aerogels that achieve many similar properties to their bulk counterparts despite a unique fabrication process necessitated by the desire to avoid supercritical drying in such thin films contained within a microdevice. Great thermal insulation properties combined with tunable mechanical properties at reduced length scales make thin film aerogels a candidate for thermal insulation in microdevices. Additionally, aerogel systems may provide a path to achieve tunable thermal properties independent of electrical properties, as is desired in thermoelectric devices. Most thermal studies and industrial applications have focused on bulk aerogel monoliths. Although interest in aerogel thin films was expressed in the 60's, it was not until the early 90's that aerogel fabrication techniques were developed to produce high porosity thin films. Active research is 
still underway in fabrication techniques for thin films, microspheres, and coatings.[18]

\subsubsection{Structure of aerogels}

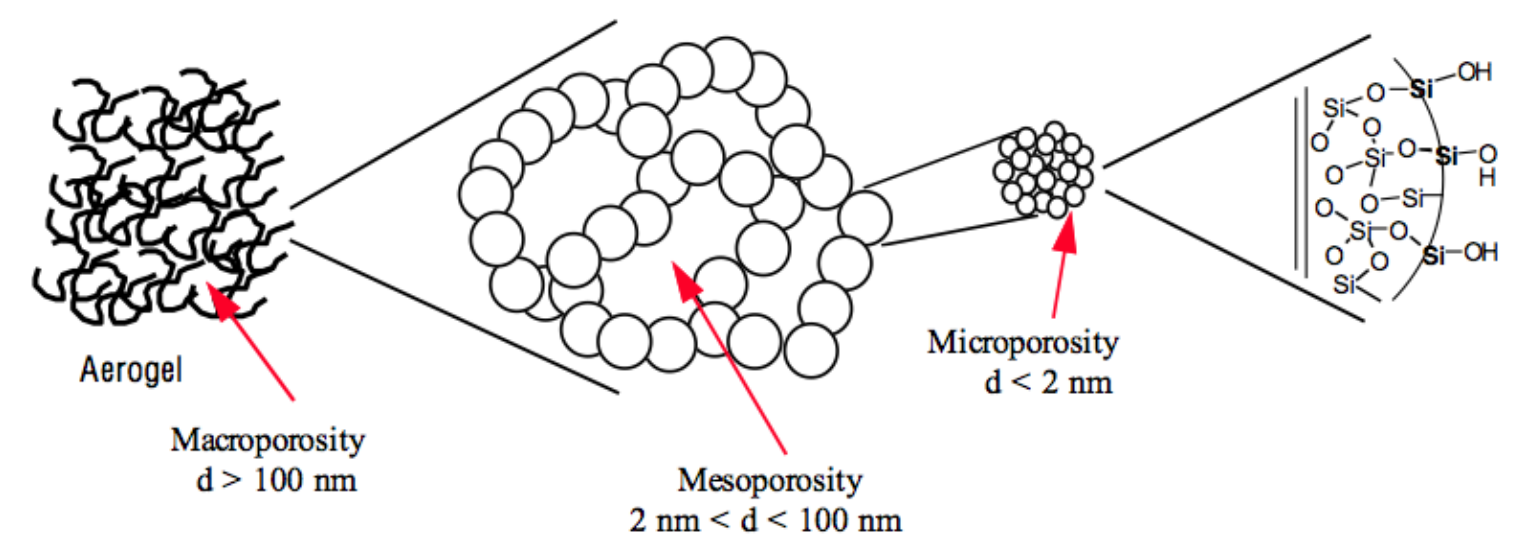

Figure 4.1: Different length scales of aerogel features.[2] The mesoporous silica chains contain clumps of silica molecules that make a random walk throughout the material system. The microporous silica clumps are capped by terminal groups. These clumps are not generally neat, geometrically defined groups. Rather, depending on the sol-gel process, they are highly fractal in nature with complex interconnects and pores between them as depicted in Figure 4.3. In thin films, the macroporous regime can be thought of as an irregularity or incomplete portion of the larger aerogel since pores in that regime are often larger than the film thickness. Therefore, for a thin-film silica aerogel to achieve high porosities, macroporous features must be avoided.

Aerogels' wide array of properties stem from their unique structure. The silica back-bone is comprised of colloidal chains of silica intertwined around and through open pores. As seen in Figure 4.1, the colloids can exhibit microporosity features on the order of a few nanometers. The bonded colloidal chains can exhibit mesoporosity characteristics between a couple nanometers and one hundred nanometers. The entire tangled network of these chains can exhibit macroporosity voids much greater than one hundred nanometers. Silica aerogel monoliths are commonly $95-99 \%$ air by volume while gels with significantly reduced porosity are referred to as xerogels.[2] The pore size and distribution is highly tunable based on the conditions of the sol-gel process - particularly the formation of the sol, the gelation, and solvent removal or drying process. Changing small factors in these 
processes can have a dramatic impact on the structural outcome.

The microporous structure of the aerogels is highly fractal. Branching and cross linking are key characteristics of the pores' mass fractal nature and are necessary to prevent highly porous aerogels from collapsing.[113-116] When this intricate cross weaving of pores does not fully occur, the features still generally maintain a surface fractal nature. However such surface fractals are more regularly identified in relatively lower porosity xerogels. [117, 118] Such cross linking may be a requisite attribute to achieving the large porosity identified in many aerogels.

\subsubsection{Aerogel fabrication}

Silica aerogels, and other metal oxide aerogels, are made through the sol-gel process. In this process, a wet gel is made as an oxide monomer solution undergoes polymerization. Polymerization conditions impact the solid-backbone structure of the wet gel. The pores in the structure are filled with a solvent such as ethanol or water. In order to create aerogels from the wet gel, the solvent in the pores must be replaced with air while retaining the highly porous gel structure. Once the solvent is removed, the dry silica backbone remains as a tangled open matrix of silica chains.[97]

Figure 4.2 depicts a standard sol-gel process. A colloidal suspension of silica undergoes a polymerization reaction forming the solid backbone of the gel network. There are a number of reaction parameters which can be varied to dictate the structural outcome of an aerogel. The reaction parameters are so interdependent that fabricating aerogels is often more of an art than a science. The fabrication of silica aerogels can be subdivided into three major steps:

1. Hydrolysis and Condensation of Silica: Silica polymerization occurs via hydrolysis and condensation reactions. Since these reactions occur simultaneously, their relative rates determine the growth of the silica chains. The structure can vary from a random walk of polymer chains to an extended 


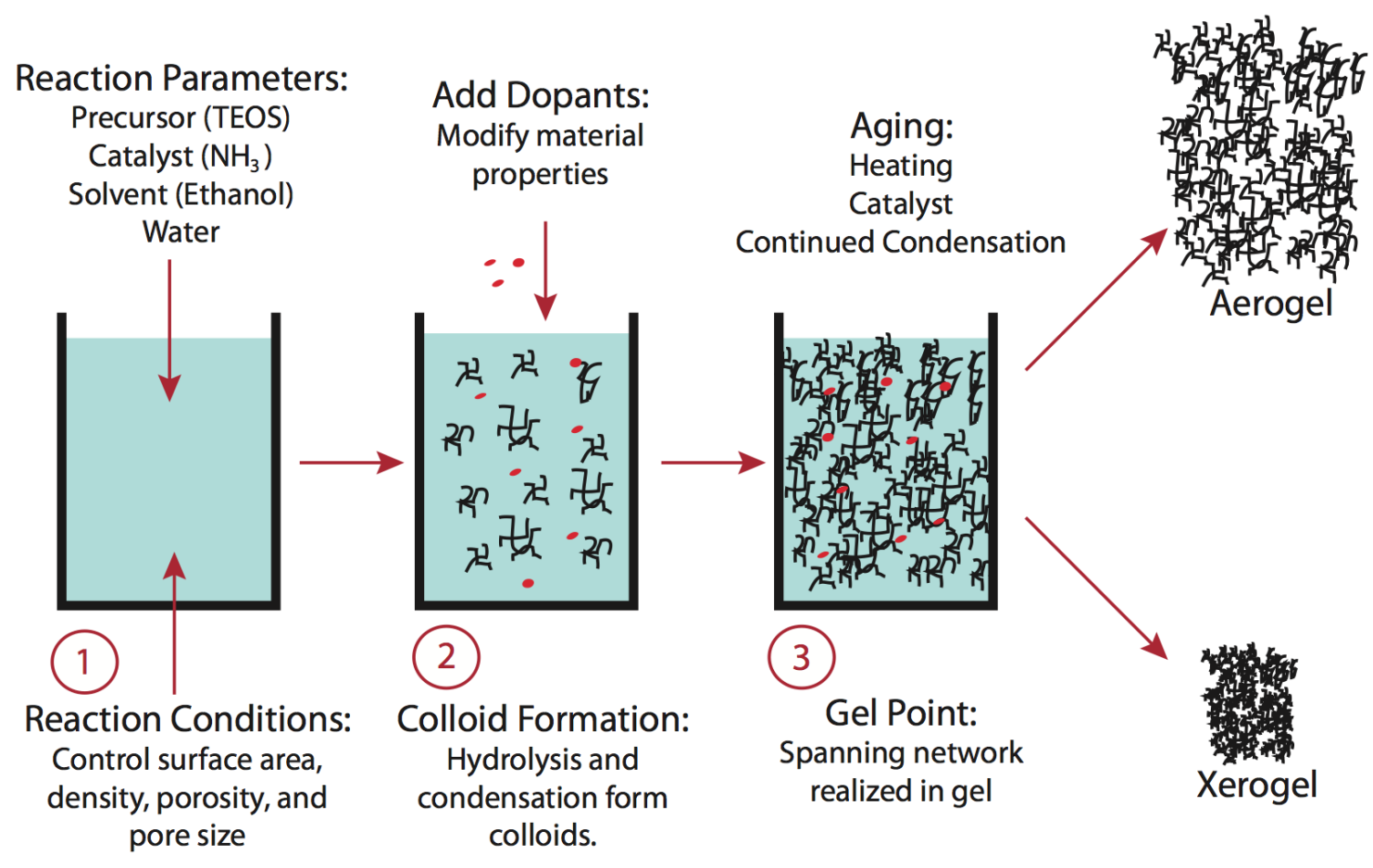

Figure 4.2: Steps and properties of a sol-gel process.[3]

rod shaped chain. Reaction dependent polymer formation was characterized by Brinker and Scherer in 1985 on xerogel samples.[119] Resulting gel properties have since been studied by other groups. $[119,120]$ The parameters most heavily influencing the relative reaction rates are the $\mathrm{pH}$ of the solution and the molecular ratio of water to silicate precursor. Generally, acid catalyzed reactions result in linear chains that are not highly branched which become physically entangled and yield larger pore sizes; whereas base catalyzed reactions result in highly branched more rigid structures with smaller pore sizes (see Figure 4.3).[119] Two-step acid-base catalysis is most commonly used to initiate the hydrolysis reaction and then induce the condensation reaction at a desired time. In this study, acid-base catalysis is used since Brinker found via SAXS examination of wet gels that two-step acid-base catalyzed systems exhibit highly branched structures that are more rigid causing less overall gel shrinkage during ambient drying.[97] The reaction 
a)

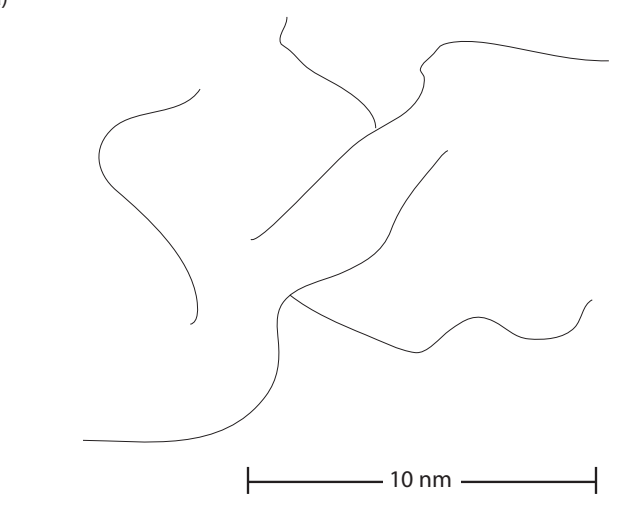

b)

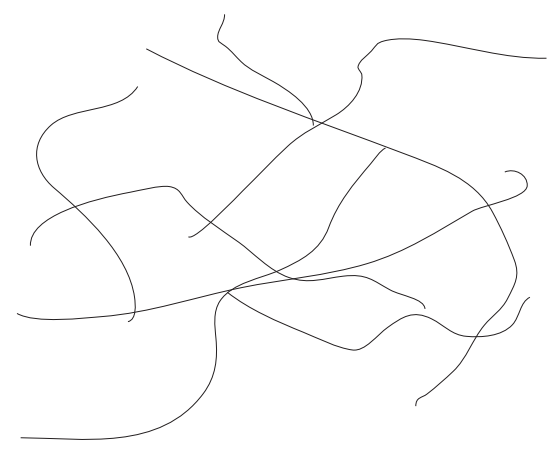

c)

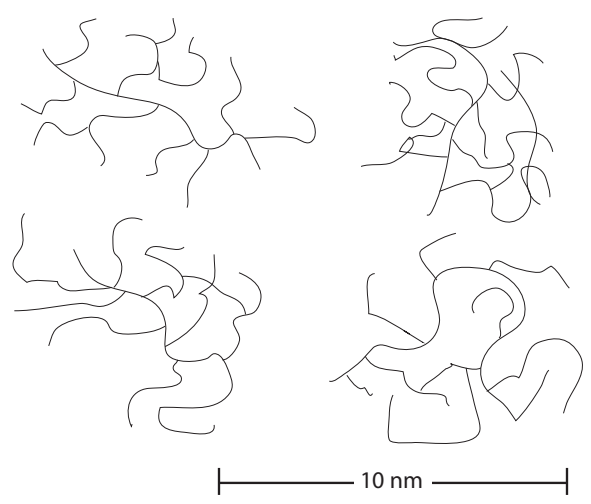

d)

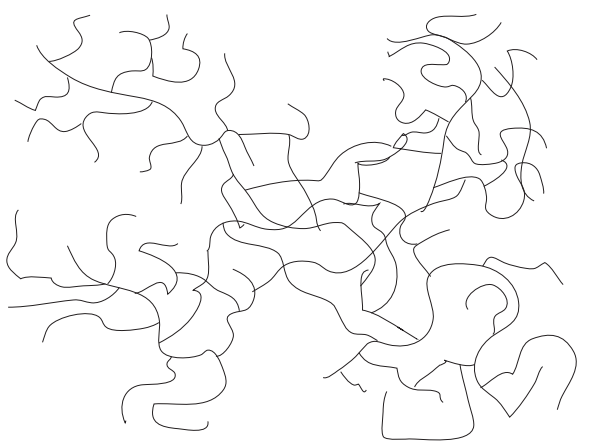

Figure 4.3: Depiction of silica network formation. Subfigure a) shows relatively linear chains of the backbone material far from the gelation point. Subfigure b) shows these same chains with greater entanglement and cross linking at the gel point. Subfigures c) and d) show a much more clustered network prior to and after reaching the gel point.

temperature, solvent, catalyst, and agitation can also impact the polymerization kinetics and impact the structure of the resultant gel.[3]

2. Modification: Terminal ends of silica chains can be modified after the gel point is reached. This is done to change the functionality of the gel structure. Modification in this study focused on yielding hydrophobic gel properties that were compatible with MEMS processing techniques. Modification for hydrophobicity essentially caps the terminal molecules lining the pores of an aerogel. The modifying agent (commonly trimethylchlorosilane, TMCS, hexamethyldisiloxane, HMDS, or hexamethyldisilazane, HMDZ) alters the extent of cross linkage of silica chains within a pore, ultimately impacting the pores' structure as shown in Figure 4.4. 


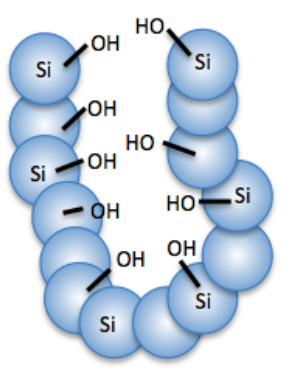

a

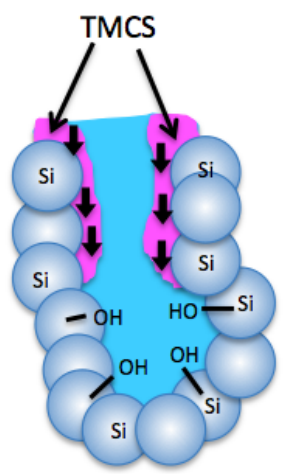

b

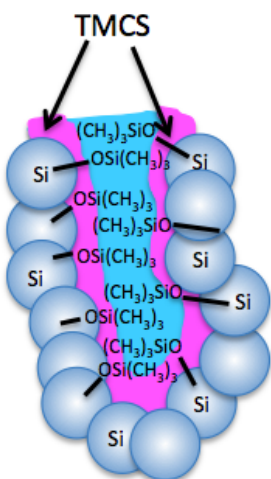

C

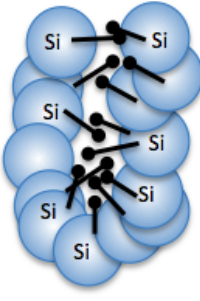

d

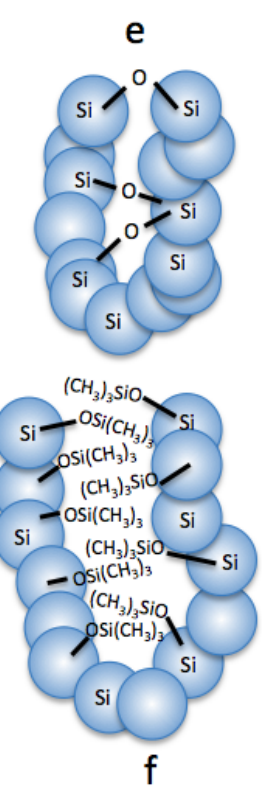

$[3]$

FiguRE 4.4: Silylation and its consequences: a) Hydroxyl groups within aerogel pores; b) Introduction of modifying agent; c) Modifying agent reacting with hydroxyl groups and replacing the capping terminal bonds; d) Surface tension causes shrinking; e) Resulting pore if no modifying agent is used in which pore interaction causes permanent siloxane bonds to form; f) Resulting pore due to effects of modifying agent with no further interaction allowing pores to spring back to near the original size

3. Drying: There are two primary methods for removing solvent from the pores of the gel structure. The first is supercritical extraction which is achieved by heating the solvent above its critical point so that the liquid-vapor interface is eliminated and the vapor can be slowly released. This method can be less than ideal for fabrication processes where simpler processes are more desirable. Furthermore, the supercritical drying process may not be successful for thinfilm aerogels adhered to a planer structure without damaging the bond between the material and substrate during drying. Alternatively, ambient drying can be employed to dry thin film systems. As the name implies, drying in this scenario occurs under ambient temperature and pressure conditions. Due to the surface tension between the liquid and solid phases of the gel, the silica structure shrinks as the solvent evaporates. Modification with hydrophobic polar molecules such 
as TMCS or HMDZ is necessary to ensure the gel will spring back to a porous structure after shrinking, rather than experiencing cross-linking and permanent shrinkage (see Figure 4.4).[3]

\subsubsection{Thermal transport studies of aerogels}

Shortly following the first fabrication of aerogels around 1930, Kistler identified the unique thermal properties of the material.[121, 122] For his aerogel samples, Kistler reported record-low thermal conductivities at atmospheric pressure, $10 \%$ below that of non-convecting air. Kistler also reported the dramatic effects gas pressure and the type of gas present have on the effective thermal conductivity. Understanding thermal transport in the solid phase of an aerogel is far from trivial. Posselt et al. performed temperature dependent thermal conductivity and specific heat capacity measurements paired with fractal cluster size measurements from small angle neutron scattering and speed of sound measurements on silica aerogels to isolate the respective temperature regimes in which phonon, fracton, or particle based transport dominate.[123] While the crossover points between these temperature regimes are dependent on the density and structure of the specific aerogel, Posselt et al. found phonon dominated transport up to $1.2 \mathrm{~K}$, fracton dominated transport between $1.2 \mathrm{~K}$ and $3 \mathrm{~K}$, and particle dominated transport at higher temperatures. This particle transport regime was first described by Einstein and is often referred to as the lower limit of thermal conductivity. It successfully describes thermal transport in amorphous materials with vibrating atoms interacting as uncoupled oscillators.[27, 28, 30] Scheuerpflug et al. showed for several aerogel samples that the ratio of an aerogel's thermal conductivity to that of vitreous silica was constant or nearly constant at temperatures above $100 \mathrm{~K}$. Additionally, a small but significant increase in aerogel specific heat compared to vitreous silica was measured at higher temperatures (tested up to $330 \mathrm{~K}$ ). They suggested that this is caused by a larger specific surface area due to dangling bonds 
allowing additional vibrational modes.[124]

Later thermal conductivity studies focused more acutely on how the microstructure of the aerogels altered thermal transport. Lu et al. determined, while studying carbon and silica aerogels, that conduction in the solid phase is controlled by sample density and the connectivity between particles. The ratio of the precursor molecule, resorcinol, to catalyst is found to control the pore size which in turn strongly influences the gas conductivity within the aerogel.[125-127] Empirical relaxation times calculated via gas dynamics and heat exchange between pores can be related to the interaction between the gas and solid phases.[128] Some more recent studies focus on unique types of aerogel and the effects of modifications to the fabrication process. Hostler et al. measured clay based aerogel thermal properties while studying the effects of pore orientation as well as the addition of a polymer to the material matrix. They found the addition of the polymer improved the materials mechanical strength while decreasing thermal conductivity.[129] Carbon aerogels with carbon nanotubes in the pores were found to double the system's thermal conductivity compared to carbon aerogels without the presence of carbon nanotubes.[130] Similarly, silica aerogels with xonotlite-type calcium silicate particles in the aerogel matrix were studied in comparison to standard monolith aerogels. This study also showed the included particles increased the sample's thermal conductivity at a given density. It has also been shown that even when the aerogel backbone is made from highly conductive material (such as carbon nanotubes), the highly porous aerogel structure can have a very low thermal conductivity.[131]

\subsection{Aerogel insulated MEMS}

In a collaboration between Honeywell, Arthur Lichtenberger's research group in the University of Virginia Microfabrication Laboratory and Pamela Norris' Soft 
Materials Laboratory and Nanoscale Energy Transport Laboratory, a research initiative was created to study thermal insulation in microelectromechanical systems (MEMS) using aerogel films. Through this initiative, we sought to formulate an aerogel capable of being embedded into a wafer while being compatible with a set of MEMS fabrication processes for potential integration of aerogel insulation into MEMS devices. Such a design could be immediately applied to specific MEMS applications, such as insulating miniature hotplates on chemical sensor chips. For

the study to be successful, thin film silica aerogels (TFSA) must be capable of competing with the current method of thermal insulation in MEMS devices in which diaphragms are used. The remainder of this section will highlight the success of the collaboration in producing usable planar aerogel fabrication on wafers, success micro fabricating on these thin film aerogels, and the measured thermal conductivity of these samples compared with currently used thermal isolation technology for micro hotplates.

\subsubsection{Sol-gel procedures}

Three unique aerogel recipes were completed by Casey Bauer. In the first process the sol-gel precursor solution was made by first combining tetraethyl orthosilicate (TEOS), ethanol (EtOH), $\mathrm{H}_{2} \mathrm{O}$ and hydrogen chloride (HCL) in molar ratios of 1.0 : $4.1: 0.9: 42 \times 10^{-6}$ at $60^{\circ} \mathrm{C}$ for ninety minutes to produce prehydrolyzed TEOS solution. Gelation was induced by combining prehydrolyzed TEOS, EtOH, and 0.15 $\mathrm{N}$ ammonia hydroxide $\left(\mathrm{NH}_{4} \mathrm{OH}\right)$ in a volume ratio of 1.0: $1.33: 2.0$. The approximate gelation time of this formulation was tuned to 3.5 hours based on the $\mathrm{NH}_{4} \mathrm{OH}$ catalyst concentration. Samples produced using this technique are referred to as Aerogel A and this fabrication process as Recipe A.

The second procedure, Recipe B, follows the B2 acid-base catalyzed method outlined by Prakash [132] and has been shown to produce aerogels with 60-90\% porosity. To make stock solutions, TEOS, EtOH, $\mathrm{H}_{2} \mathrm{O}$, and HCL were combined in 
molar ratios of $1.0: 3.8: 1.1: 7 \times 10^{-6}$ at $60^{\circ} \mathrm{C}$ for ninety minutes. Once cooled to room temperature, the stock solution, $\mathrm{EtOH}$, and $0.05 \mathrm{M} \mathrm{NH}_{4} \mathrm{OH}$ were combined to induce the condensation reaction and gelation in an oven at $50^{\circ} \mathrm{C}$ for a minimum of ninety hours. Such samples are referred to as Aerogel B.

The third sol-gel method (Recipe C) was a much faster process than that used to produce Aerogel A or Aerogel B. The hydrolysis reaction was achieved by combining TEOS, water, and either nitric acid or sulfuric acid and acetic acid in molar ratios of $1.0: 4.72: 3.6 \times 10^{-3}$ and $1.0: 4.72: 1.25 \times 10^{-3}: 0.035$ respectively. The solution was stirred vigorously while mixing in order to drive a homogeneous reaction by overcoming the immiscibility at such concentration. After 1.5-2.0 hours, the solution clarified, indicating that the clear sol had been obtained without precipitation of large silica particles. The further hydrolysis reaction was allowed to continue for 2-3 hours while stirring and storing in a sealed container. The condensation reaction was induced as the prehydrolyzed TEOS was added to the EtOH and $14.8 \mathrm{~N} \mathrm{NH}_{4} \mathrm{OH}$, in a volume ratio of $1: 3.12: 0.046$. Gel times of $5-8$ minutes were recorded. The variation was due to the aging of prehydrolyzed TEOS. Samples produced using this much faster process are referred to as Aerogel C.

\subsubsection{Thin-film deposition processes}

Aerogel A and Aerogel C films were deposited using thin film deposition methods of both spin and dip coating.[133] During the condensation reaction, the non-viscous solutions used to prepare Aerogel A and Aerogel C were applied to the silicon dioxide wafers 45 and 4 minutes, respectively, before gelation and allowed to gel. During deposition onto the wafers, care was taken to prevent solvent evaporation, which would collapse the porous structure. The gel and wafer were then washed in aging solutions to modify the gels, strengthening them and making them hydrophobic. Aerogel A was treated for ambient drying, which included a 30 minute wash in excess EtOH, another 30 minute wash in hexane, a minimum 12 
hour wash in 6\% TMCS and 94\% hexane, then another 30 minutes in hexane and finally 30 minutes in EtOH. Aerogel $\mathrm{C}$ was deposited onto the wafer and allowed to gel. It was then washed in EtOH for 2 hours and modified in a solution of $14.8 \mathrm{~N}$ $\mathrm{NH}_{4} \mathrm{OH}, \mathrm{EtOH}$, and hexamethyldisilazane (HMDS) in a volume ratio of $1: 17: 3$ at $55^{\circ} \mathrm{C}$ overnight. The gels were then returned to room temperature and washed for 2 hours in excess EtOH before drying. The HMDS modification produced hydrophobic gels.

Alternatively, a sonication deposition method developed by Prakash and Brinker [134] was used to deposit Aerogel B. The gel is first washed for three hours in EtOH, then for three hours in hexane, and then reacted with a solution of $6 \%$ TMCS or HMDS and 94\% hexane by volume to replace the hydroxide groups in the pores with $\mathrm{O}-\mathrm{Si}(\mathrm{Me})_{3}$. The six-hour washing process was then repeated. The modified gel was sonicated at an output of $20 \mathrm{~Hz}$ for at least 20 minutes then diluted in EtOH to form a sol-gel suspension. The suspension was then filtered through a $1.1 \mu \mathrm{m}$ syringe filter and deposited on the wafer to form the aerogel film. Initial samples were not washed in $\mathrm{EtOH}$ prior to sonication and were sonicated in 100\% excess hexane. This however, produced samples with large roughness which did not allow the subsequently applied capping layer to sufficiently planarize the surface. A subset of the Aeorgel B films was pyrolized at $400^{\circ} \mathrm{C}$ for 1 hour as described by Parkash et al.[132] to increase porosity and lower thermal conductivity (referred to as Aerogel B-Pyrolyzed).

\subsubsection{Microfabrication on the samples}

With the motivation of inserting TFSA into MEMS, as well as a need to pattern heating pads onto our samples to perform thermal conductivity tests, methods to successfully microfabricate on the samples needed to be determined. While some aerogels are fairly durable, such as carbon based aerogels, silica aerogels are very brittle and can easily collapse. Care had to be taken to avoid destroying the aerogel samples while fabricating them into devices.[135] Initial studies for compatible 
microfabrication processes, as well as initial successful fabrications, were completed by Professor Lichtenberger's research group, with Roy Mathews successfully fabricating on many of the samples. Due to the fragile nature of silica aerogel, samples were sealed with a $200 \mathrm{~nm}$ silicon dioxide protective capping layer deposited through magnetron sputtering at $300 \mathrm{~W}$ and 4 mTorr pressure. Side view scanning electron microscope images were used to confirm no significant damage was done to the aerogel film during this processes (see Figure 4.10).

Initially a $30 \mathrm{~nm}$ gold layer with a $5 \mathrm{~nm}$ titanium adhesion layer was deposited onto the sample and a portion removed using a chemical wet etch process. A positive photoresist was spin coated onto the sample. After exposure under a serpentine shaped photolithography mask, the serpentine feature is protected by the photoresist during the etch. The wet etch used an iodine based solution for 45 seconds and buffered hydrofluoric etchant for 15 seconds. While the etch removed a portion of the gold and titanium layer as desired, it also damaged the surface of the $\mathrm{SiO}_{x}$ layer introducing pinholes that protruded into the aerogel.[135] This wet etchant process also likely damaged the aerogel and capping layer from the sides of the sample, further diminishing it's potential to be used to process aerogel films.

Alternatives were considered for the metal and etching processes while accounting for the delicate nature of the sample. A reactive ion etch of a niobium metal layer was ultimately selected. Reactive ion etching (RIE) allows a more selective etching process than alternatives. Niobium reactions with specific plasmas are well known and available in the UVML. Additionally, niobium readily adheres to $\mathrm{SiO}_{2}$. This layer was also deposited using magnetron sputtering at $500 \mathrm{~W}$ in 4.7 mTorr argon environment. The primary disadvantage of niobium compared to other metals (such as platinum) is a somewhat lower phase change temperature that prevents ultra high temperature testing with $3 \omega$.

In preparation for photolithography, the sample was cleaned and positive photoresist AZ 4110 was applied via spin coating. The UVML contact mask aligner 


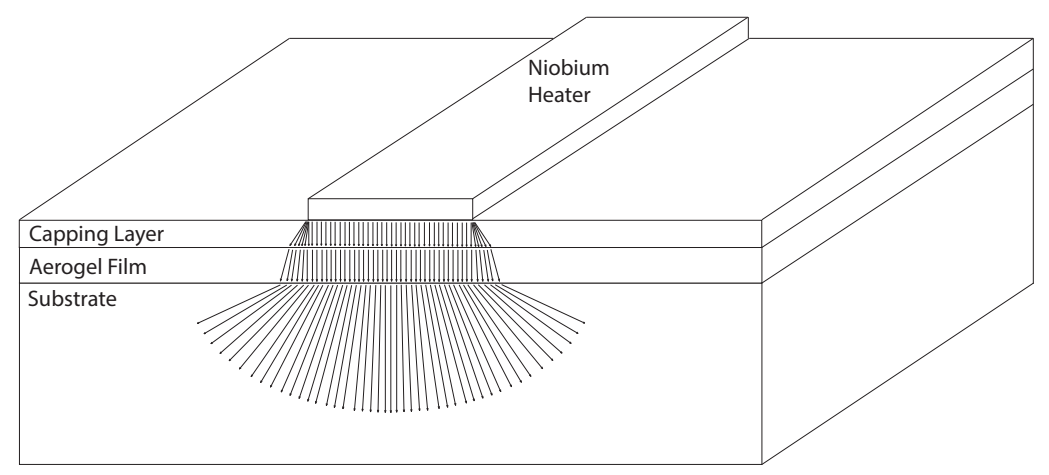

Figure 4.5: Schematic of the central portion of the sample after micro fabrication. Heat travels from the metal wire through each layer of the sample.

MJB4 was used to expose the photoresist to the mask designed for $3 \omega$. The sample consisting of the substrate, aerogel layer, $\mathrm{SiO}_{x}$ capping layer, niobium metal, and photoresist post exposure was then loaded into the Oxford Instrument's RIE. For etching, a sulfur hexaflouride $\left(\mathrm{SF}_{6}\right)$ and argon chemistry were used. This tool allows for laser endpoint detection to minimize over etching of the wire or sample surface. After completion, the sample underwent an oxygen ash to remove the protective photoresist above the remaining metal wire. This process was repeated on samples without the aerogel present to serve as a control. ${ }^{1}$

\subsubsection{Implementation of $3 \omega$ and sample thermal conductivity measurements}

The initial design and implementation of the $3 \omega$ thermal characterization technique in UVA's nanoscale heat transfer laboratory was pursued specifically for the aerogel measurements in this study. Many of the details of this experimental system are discussed in Chapter 3. Data was taken at driving frequencies from 10

\footnotetext{
${ }^{1}$ As mentioned, Casey Bauer and Roy Mathews were instrumental in creating these samples and preparing them for $3 \omega$ tests. Beyond this, both spent a great amount of time training me to recreate and modify the methods they used. This was critical for the continuation of this study described in Section 3. In short, the success of this chapter would not have been possible without their collaboration.
} 


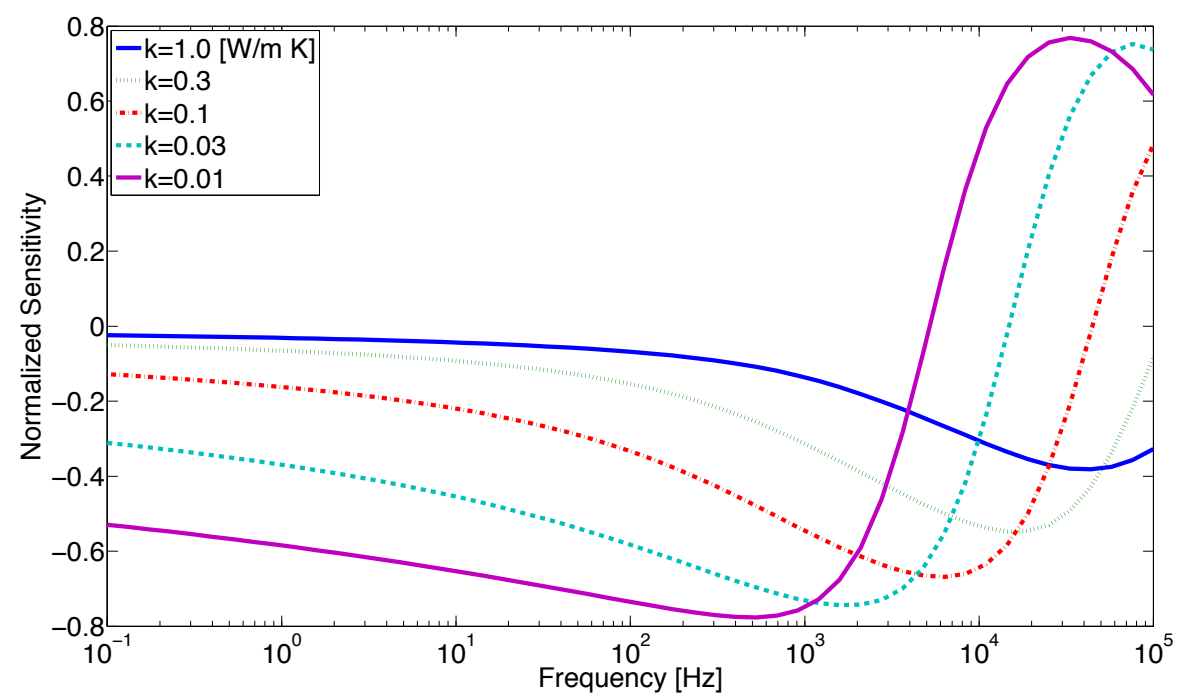

Figure 4.6: $3 \omega$ sensitivity plot to an aerogel's thermal conductivity for the described three layer scenario. Sensitivity response is generated for the outer bounds of likely thermal conductivity (1 $\mathrm{W} / \mathrm{m} \mathrm{K}$ being near that of amorphous $\mathrm{SiO}_{2}$ and $0.01 \mathrm{~W} / \mathrm{m} \mathrm{K}$ is reasonably less than non convective air) as well as three intermediate scenarios. In generating these plots the assumed aerogel density (and therefore volumetric heat capacity) is varied from assumed $\mathrm{SiO}_{2}$ fill fraction of $100 \%$ to $1 \%$ to more reasonably match potential measured samples. Input aerogel thickness is $1 \mu \mathrm{m}$.

$\mathrm{Hz}$ to $10 \mathrm{kHz}$. The thermal conductivity of the sample substrate and the capping layer were independently verified. Figure 4.5 schematically depicts the experimental sample as heat flows from the metal heating line through the 3 layer system (silica capping layer, aerogel sample, and silica substrate). $3 \omega$ type experiments are rarely used on 3 layer systems, although many publications suggest their hypothetical usage. $[74,93]$

The solution to the heat diffusion equation for such a three layer system as well as an overview of sensitivity analysis are provided in Chapter 3. Figure 4.6 provides the sensitivity response of the experiment to the thermal conductivity of the aerogel film for the broad range of potential thermal conductivities the aerogel could have. In the low frequency range in which the sensitivity trend is linear, the system is behaving very similarly to the limiting scenarios in which the temperature off set method used in earlier implementations of $3 \omega$ on multilayer systems could be used to determine a film's thermal conductivity.[68] At higher frequencies, an increasingly 


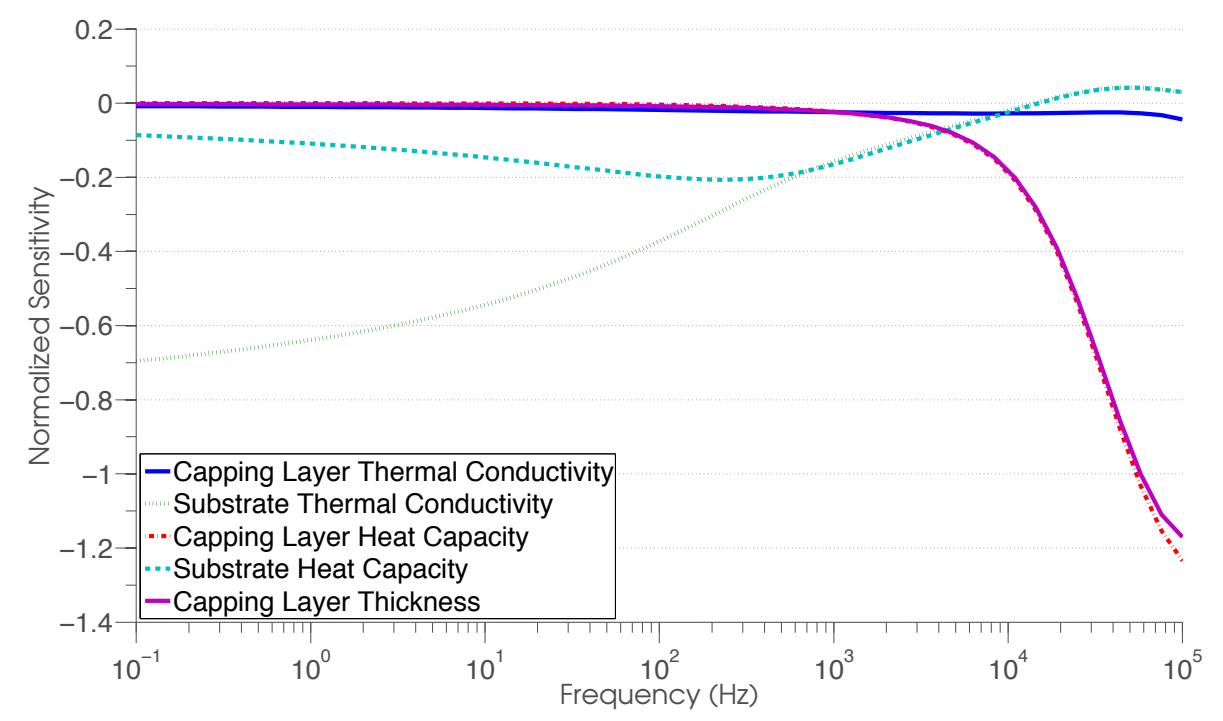

FIGURE 4.7: Experiment sensitivity to the input parameters of the 3 layer system.

larger percentage of the thermal diffusion is occurring in the film rather than the substrate. For such a thermally insulating film, at much higher frequencies the experiment will eventually become completely insensitive to properties of the substrate.[93] For the sake of comparison, Figure 4.7 shows the normalized sensitivity of the experiment in the moderate scenario (with an aerogel thermal conductivity of $0.1 \mathrm{~W} / \mathrm{m} \mathrm{K}$ ) for each of the input parameters. The properties of the capping layer only become apparent at high frequencies. The method is most sensitive to the substrate's thermal conductivity at lower frequencies. Somewhat fortuitously, in the region around $1 \mathrm{kHz}$ where sensitivity to the aerogel's thermal conductivity is at a maximum, other input parameters have a much weaker influence on the signal, giving further confidence to fitting results.

Five unique samples were measured at room temperature under a vacuum of 1 mTorr: Samples A, B, B-pyrolized, C, and a second version of aerogel C in which the thickness was doubled. Each of these films were ambiently dried. Recipe C was also used to make a monolithic aerogel using supercritical drying, and attempted with supercritical drying of a thin film. Supercritical drying destroyed the thin film version 
of Recipe C. Figure 4.9 shows a comparison of the thin-film, ambiently dried Recipe C aerogel compared to the supercritically dried bulk version of the recipe. Figure 4.8 shows raw data converted to $T_{2 \omega}$ for both a blank substrate, and the three layer Aerogel B sample. The best fit results of the five samples measured are displayed in Table 4.1.

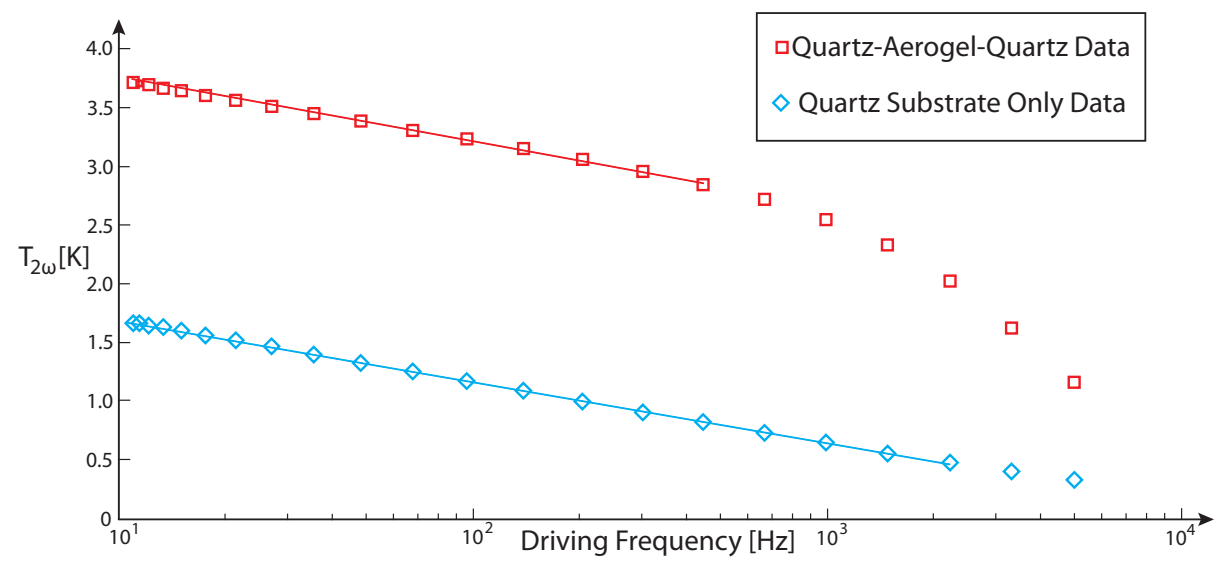

FiguRe 4.8: Exemplary raw data converted from in-phase $V_{3 \omega}$ to in-phase $T_{2 \omega}$ for both an amorphous $\mathrm{SiO}_{2}$ substrate, and sample Aerogel B. Fit lines show region in which the slope method can be used to determine sample thermal conductivity.

TABLE 4.1: Thermal Testing Results

\begin{tabular}{cccc}
\hline $\begin{array}{c}\text { Aerogel } \\
\text { Type }\end{array}$ & $\begin{array}{c}\text { Thickness } \\
(\mathrm{nm})\end{array}$ & $\begin{array}{c}k_{\text {film }} \\
(\mathrm{W} / \mathrm{mK})\end{array}$ & $\begin{array}{c}\text { Estimated Error } \\
(\mathrm{W} / \mathrm{mK})\end{array}$ \\
\hline A & 1280 & 0.24 & \pm 0.05 \\
\hline B & 250 & 0.067 & \pm 0.01 \\
\hline B-pyrol & 370 & 0.024 & \pm 0.005 \\
\hline C & 300 & 0.33 & \pm 0.07 \\
\hline C & 620 & 0.29 & \pm 0.06 \\
\hline
\end{tabular}

Successfully creating highly porous aerogels without the aid of supercritical drying proved challenging. The relatively high thermal conductivity results from the $3 \omega$ testing of films created from Recipe $\mathrm{A}$ and Recipe $\mathrm{C}$ indicate that too much solvent evaporation occurred during synthesis causing the structure to collapse. This was inspite of care being taken to minimize solvent evaporation during gelation 

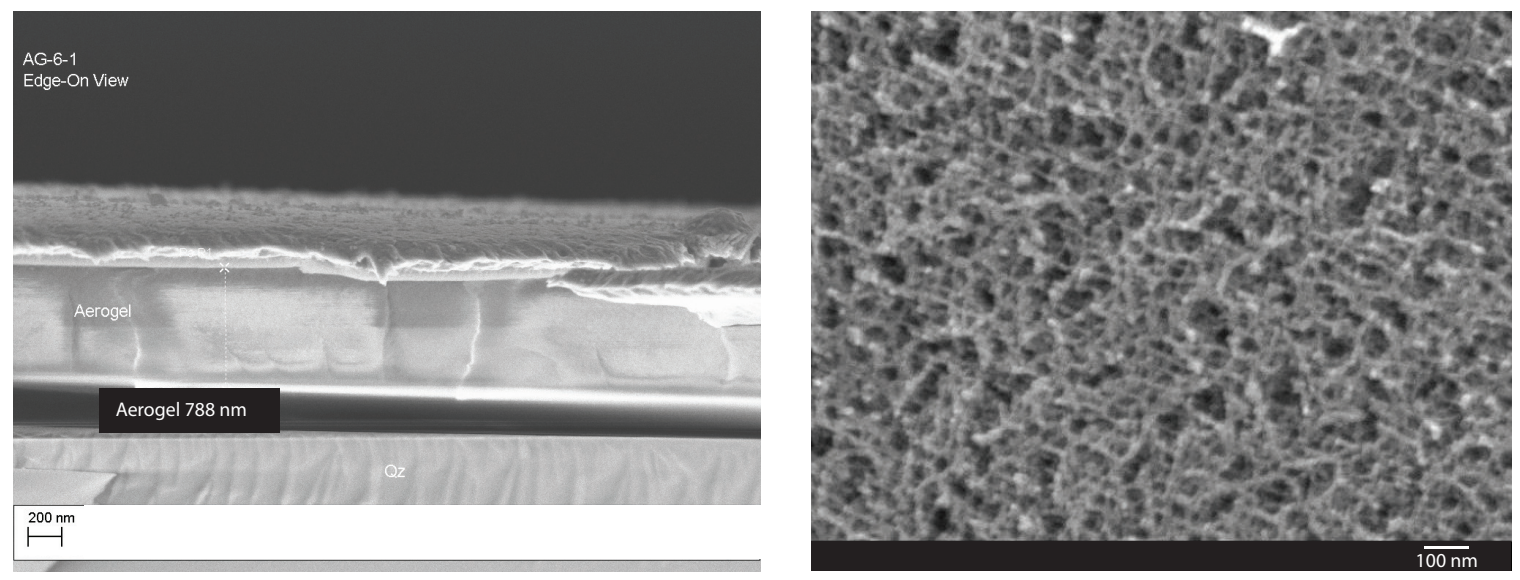

FiguRE 4.9: Left: side view SEM image of an aerogel thin film formed through process C. Right: bulk aerogel formed through process C. These images confirm that aerogel C's porous structure collapsed during deposition.

and modification. The lower than sought porosity of Sample C was verified by comparing SEM images of a bulk version of Aerogel $\mathrm{C}$ and a thin film version similar to the samples tested, as shown in Figure 4.9. The bulk sample produced from Recipe $\mathrm{C}$ showed highly porous silica networks while the thin film did not display this feature.

The sonication method used on Aerogel B provided the most robust method to combat solvent evaporation and structural collapse due in part to gel modification being completed prior to deposition. Aerogel B yielded a lower conductivity than either A or C. This value was further minimized by the pyrolization heat treatment process. The pyrolized version of Aerogel B yielded a thermal conductivity of 0.024 $\mathrm{W} / \mathrm{m} \mathrm{K}$. The $400^{\circ} \mathrm{C}$ pyrolization likely oxidized any remaining organic groups yielding a more porous structure. This was further verified by the top and side view SEM images of Aerogel B-Pyrolized shown in Figure 4.10.

The engineering objective of this research was to determine the thermal applicability of TFSAs as alternatives to diaphragm insulation in microdevices. Collaborators at Honeywell report current vacuum sealed diaphragm technology has a thermal conductance of $12 \mathrm{~kW} / \mathrm{m}^{2} \mathrm{~K}$ on wafers several hundred microns thick. By extrapolating the measured thermal conductivity results of Aerogel B-pyrolized, it 

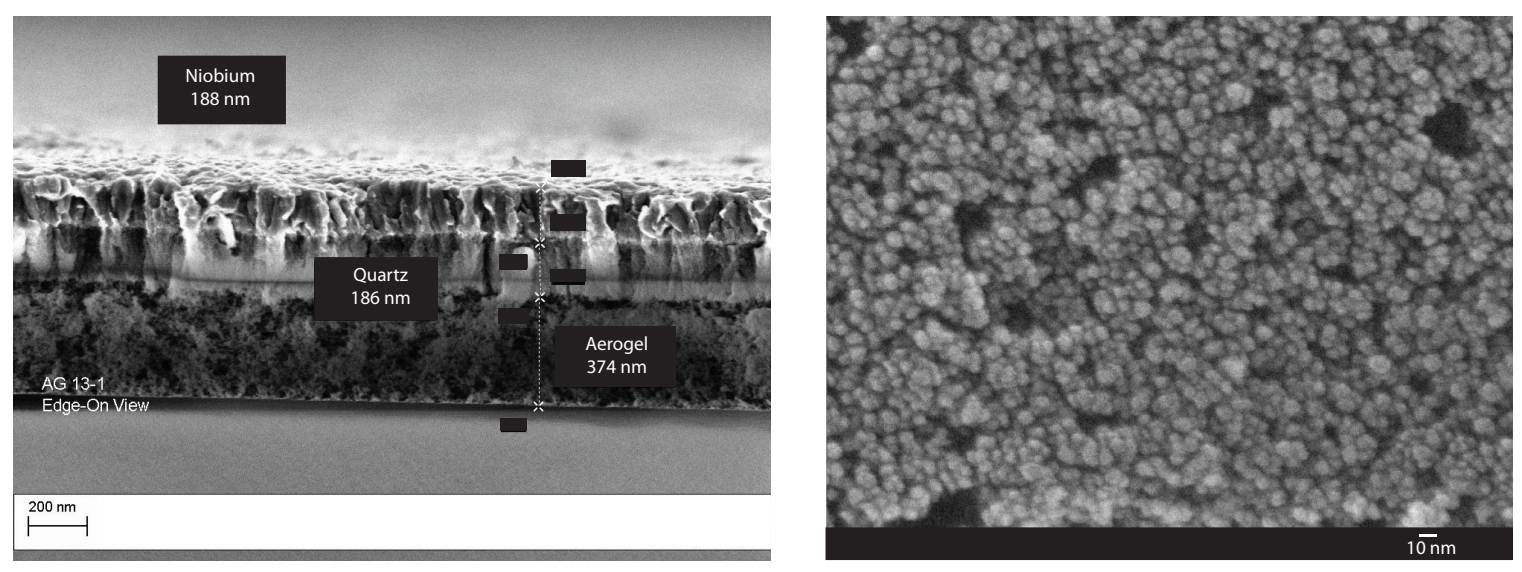

Figure 4.10: Left: side view of Aerogel B-pryolized after deposition of the quartz and niobium layers. Right: high resolution top view of the same sample prior to the deposition of capping layers. Both images show the highly porous structure of the aerogel remained in tact after deposition of the gel and of the two capping layers.

can be inferred that a 2 micron thick evacuated aerogel would match this performance, and superior performance could be achieved at greater thickness while requiring a fraction of the volume as the diaphragms. Aerogel A and Aerogel $\mathrm{C}$ would match the diaphragm performance at thicknesses less than $30 \mu \mathrm{m}$. Aerogel A may be preferable due to the relative simplicity of the recipe used. Figure 4.11 shows a test wafer micropatterned with heating elements above an aerogel film (with a protective capping layer). The aerogel film in this figure was deposited by the Soft Materials Laboratory while Honeywell added the additional metal pattern and elements on the surface. This image depicts the major fabrication accomplishments of this project: recreating the unique microstructure of a silica aerogel as a thin-film which supports the circuit elements and micro heaters as needed by chemical sensors and other thermal isolation applications.

\subsection{Structural tuning study}

The study in the previous section proved successful in achieving the direct engineering and application focused objectives. However, to advance these results into a general understanding of thermal transport in TFSAs, a follow-up study was 


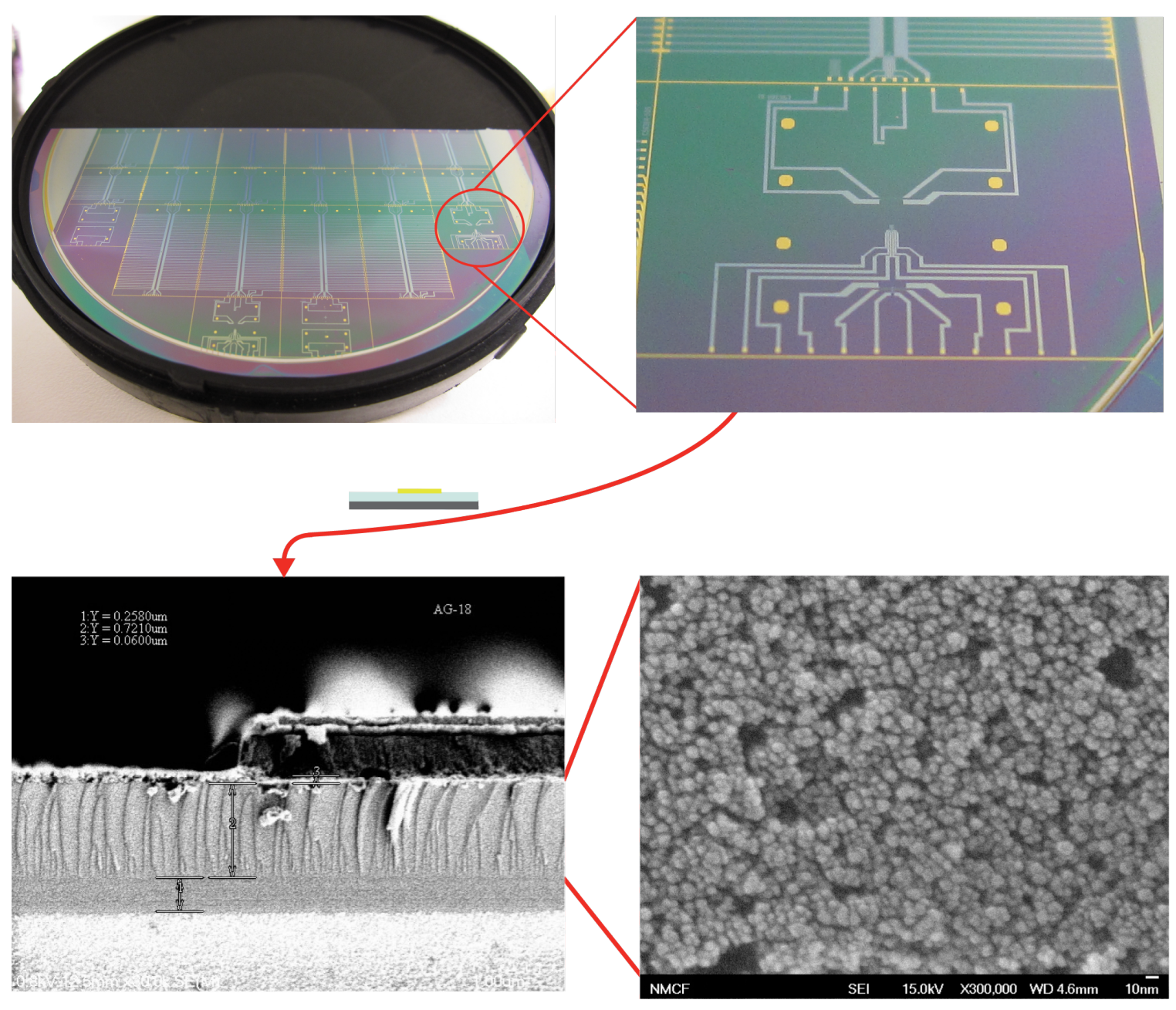

FIGURE 4.11: Implementation of a TFSA into a micro fabricated circuit.

developed. In line with the broader theme of this dissertation, I sought a series of aerogel samples with systematically altered structural properties. To that end, in collaboration with Casey Bauer, methods of tuning the sample recipes which, according to literature, could produce nuanced changes in the aerogel's structure beyond the more general change in porosity were implemented. In aid of this pursuit, small angle x-ray scattering was used as the primary tool to quantify these structural changes. Additionally, x-ray reflectivity aided in determining the films' thickness and density (and therefore porosity). The successful micro fabrication process and thermal characterization previously demonstrated were repeated for each sample in the study. All thermal characterization reported was acquired with 
the sample at a temperature of $300 \mathrm{~K}$ and in a vacuum environment to isolate the role of thermal transport in the silica backbone of the system.

\subsubsection{Recipe design}

With the successful implementation of Recipe B proven, each aerogel in this study used that formulation as a starting point with controlled modifications. All fabrication parameters not further discussed are identical to Recipe B. Four methods of tuning aerogel properties were explored. Each of these modifications can cause several interdependent changes in the reactions kinetics or the internal stress of the system. Therefore, it is not expected that each modification will have an apparent individual feature which it modified. Alternatively, each parameter change may have a domino effect. Regardless, the objective is to create quality aerogels with unique structural features (porosity, pore size, and fractal features) for comparison. The four recipe modifications are:

\section{- Base catalyst concentration $\left(\mathrm{NH}_{4} \mathrm{OH}\right)$}

The timing of the introduction as well as the concentration of the base catalyst can alter when the gel starts forming colloidal branches in a two step acid-base process. Stronger base concentrations result in a quicker gelation time.[136] Increasing the amount of base catalyst may generate smaller pores due to increased branching as depicted in subfigures c) and d) of Figure 4.3. If there is not sufficient base, the condensation reaction may not go to completion resulting in less interconnection, as shown in subfigures a) and c) of the same figure. The base concentration was varied from the $185 \mu \mathrm{L}$ used in Recipe B to as low as $80 \mu \mathrm{L}$ and as high as $220 \mu \mathrm{L}$.

\section{- Modifying agent (TMCS and HMDZ)}

The chemical used as the modifying agent alters the chemical capping the pores as well as the pore cross linkage and spring back during the drying 
phase. TMCS, used in the Aerogel B recipe in the previous section, is more commonly used in fabricating TFSA. However a study on TFSA for infrared imaging by Ruffner et al. demonstrated noticeably different structural properties when comparing TMCS to HMDZ.[100] Particularly, greater surface roughness, greater thickness, and higher porosities were noted in the aerogels modified with HMDZ. Other works on silica aerogels have also indicated HMDZ creates smaller pores and a higher porosity system.[137] Such structural deviations are sought for this study. Therefore, approximately half of the samples created used HMDZ for the modifying agent.

\section{- Acid catalyst molecule (variation to $\mathrm{HNO}_{3}$ )}

The type of acid primarily alters the rate and extent of the hydrolysis reaction. This can broadly be understood as the length of the polymer chains. It can also alter the amount of water precipitant in the pores which, in turn, can change the rate and extent of condensation (governed by the later addition of the base catalyst). This influences the degree of branching and cross linkage[119] (shown in Figure 4.3) and may significantly alter the fractal dimension of the network. The vast majority of TFSAs formed from a two step acid-base process use $\mathrm{HCl}$ as the acid. Aerogels in this study are fabricated with $\mathrm{HCl}$, with the exception of two recipes attempted with $\mathrm{HNO}_{3}$.

\section{- Heat treatment}

Heat treatment can alter the underlying structure of the sample after drying is completed. Heat treatment up to $500^{\circ} \mathrm{C}$ has been shown to increase the specific surface area of silica aerogels.[138] In this temperature range, others have observed that the heat treatment can cause changes in both the fractal dimension and pore radius but at heat treatment temperature, HTT, above $500^{\circ} \mathrm{C}$ the pores lose their fractal nature.[116] For most of the fabricated samples, a pyrolized counterpart is created. Unless otherwise noted 
pyrolization occurs at $400^{\circ} \mathrm{C}$ for one hour. The exception to this is a series of heat treated samples which include a sample with a $300^{\circ} \mathrm{C}$ HTT as well as a sample heat treated at $400^{\circ} \mathrm{C}$ for two hours.

The full list of samples fabricated and their properties appears in the Results and Discussion section in Table 4.2 .

\subsubsection{Structural characterization: SAXS and XRR}

Small angle diffuse scattering of x-rays or neutrons (SAXS and SANS, respectively, or more generally just SAS, small angle scattering) has been used for nearly a century to study density inhomogeneities in bulk systems.[139-141] SAXS data presented here was taken through the user program at Oak Ridge National Lab's Center for Nanophase Material Sciences. Dr. Andrew Payzant worked to familiarize me with the tool and alignment procedures. SAS has been employed to study monolith aerogels $[97$, 114] and thin film aerogels.[117] The method serves as a strong compliment to electron microscopy techniques. Where microscopy provides a detailed view of an individual feature, small angle scattering measurements provide a statistically significant average occurrence of some feature. SAS is particularly useful in materials in which brittleness or liquid crystals hamper electron microscopy measurements.[142]

In a SAXS experiment testing a bulk sample, an incident x-ray beam (either point or line collimated) is directed into the sample of interest. A detector is placed beyond the sample to measure the intensity and location of exiting photons. The primary beam passing directly through the sample, as well as any coherent refraction of the beam beyond the sample is blocked. The remaining diffuse scattering of the incident beam in the sample is measured. The exit angle is considered in terms of the wave form vector: $q=4 \pi / \lambda \sin (\theta)$ with the x-ray wavelength $\lambda$. At smaller angles and therefore smaller $q$, the detected relative intensity, $I$, gives information related to larger features. By investigating small angles, features on the order of several nanometers can be discerned. When the difference in electron density between the 


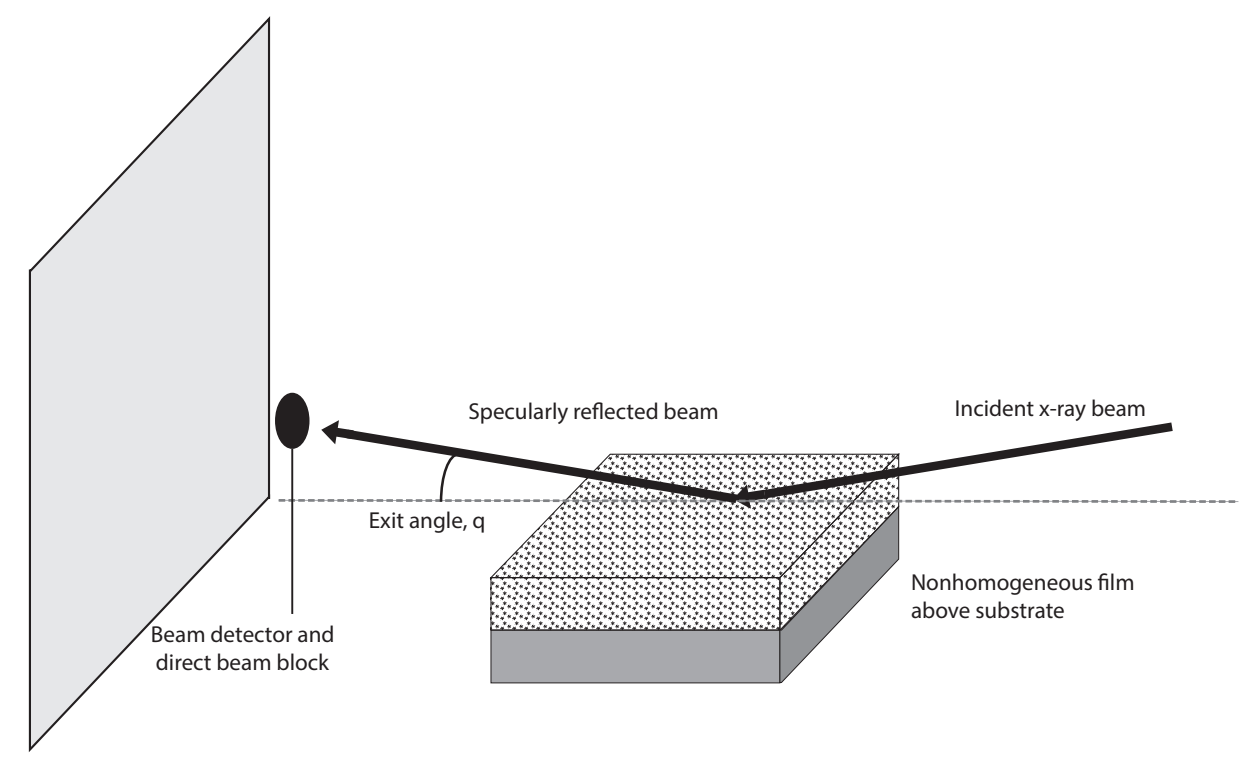

FIGURE 4.12: Grazing incidence small angle x-ray scattering (GISAXS) data collection geometry. Incident beam scatters with the sample. A beam blocker is positioned to block the specular reflection of the beam. Diffuse scattering intensity is measured as a function of the exit angle with the sample. Sizes are not to scale.

two phases within the system is large, the feature signal is easier to detect, as is the case in porous materials.

Freltoft et al. utilized SANS to measure suspended aerogel particle aggregates over a broad porosity range and determined the average pore radius of gyration (1.9$2.4 \mathrm{~nm})$, the correlation length between pores $(10-56 \mathrm{~nm})$, as well as the fractal dimension which was in the mass fractal regime on all samples. They also found the lower porosity gels exhibited more disk shaped pores.[143] SANS was employed by Reidy et al. and used to infer that varying the catalyzing agents would generate unique microstructures which could be attributed to gelation kinetics.[115] A heat treatment study on the nanostructure of xerogels using SAXS was performed by Vollet et al. This study indicated that maximum heat treatment temperature drastically altered the fractal nature of the network. Heat treatment temperatures above $500^{\circ} \mathrm{C}$ 


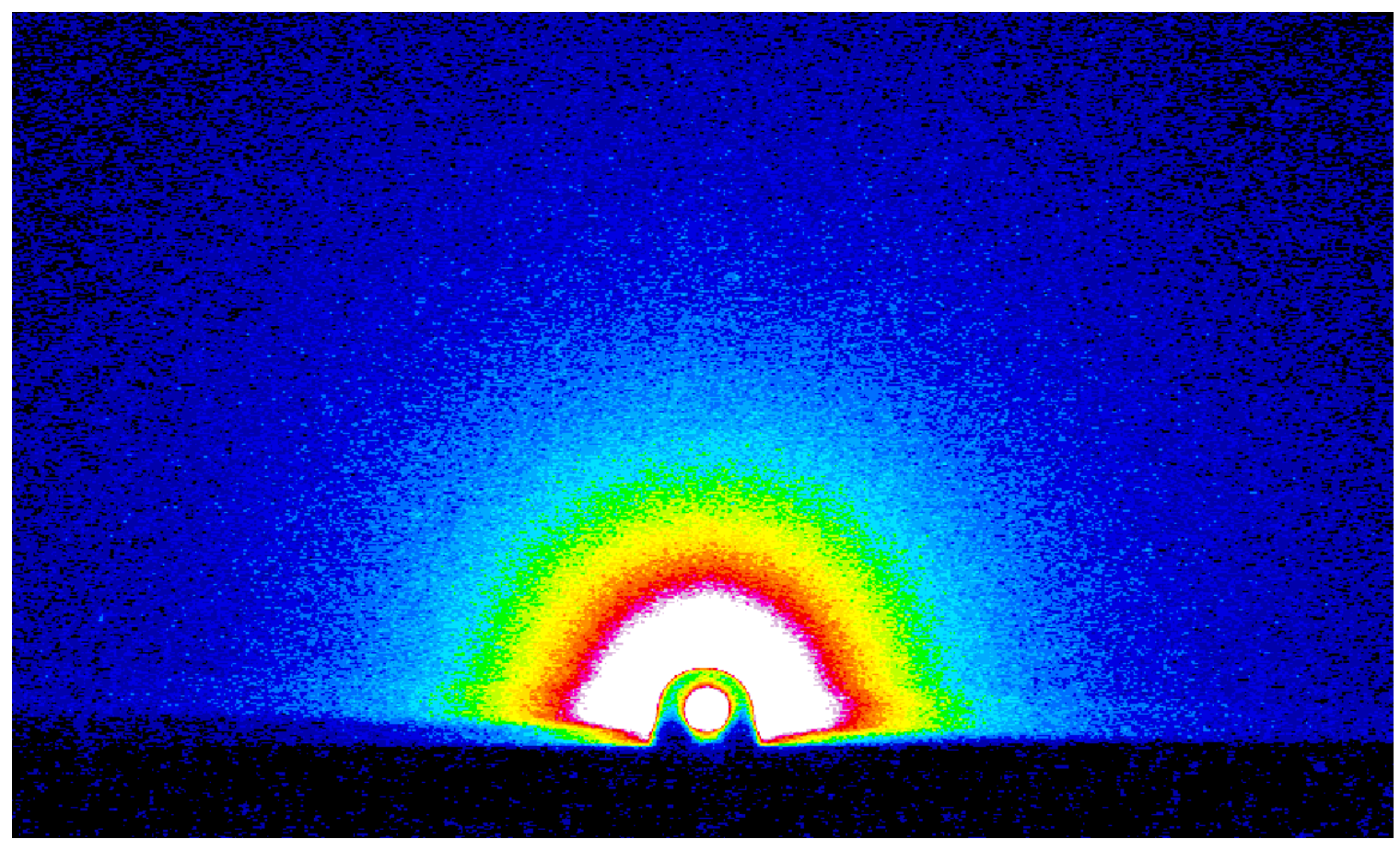

FiguRE 4.13: Example of GISAXS raw data taken on an aerogel thin film over a 10 hour period. The specularly reflected portion of the beam is blocked, although a small portion still appears on the detector. Outside of this blocked beam is an intensity profile of collected diffusely scattered x-ray signal which contains key information about pore properties.

were shown to generate smooth surfaces (Porod exponent of 4) implying the tortuous interconnects of the silica backbone had been fully removed from the structure.[116] A grazing incidence SAXS (GISAXS) study on xerogel films was performed by Hsu et al. The researchers successfully determined the average shape and size of the pores within the film (disk shaped with a mean disk thickness of $2.8 \mathrm{~nm}$ ) as well as a Porod exponent of 3.5 indicating surface fractals.[117, 144]

Here, similar measurement methods and data reduction methods are used to determine the features of the thin film aerogels studied. SAXS data was acquired using Anton Paar's SAXSess $\mathrm{mc}^{2}$ tool operating at $40 \mathrm{kV}$ and $30 \mathrm{~mA}$ with the incident beam point collimated. Alignment was performed using a digital charge couple device (CCD) detector. The sample was positioned on a digitally controlled six degree of freedom stage which provided highly repeatable and precise sample alignment control. Sample data was collected using photostimulable phosphor 


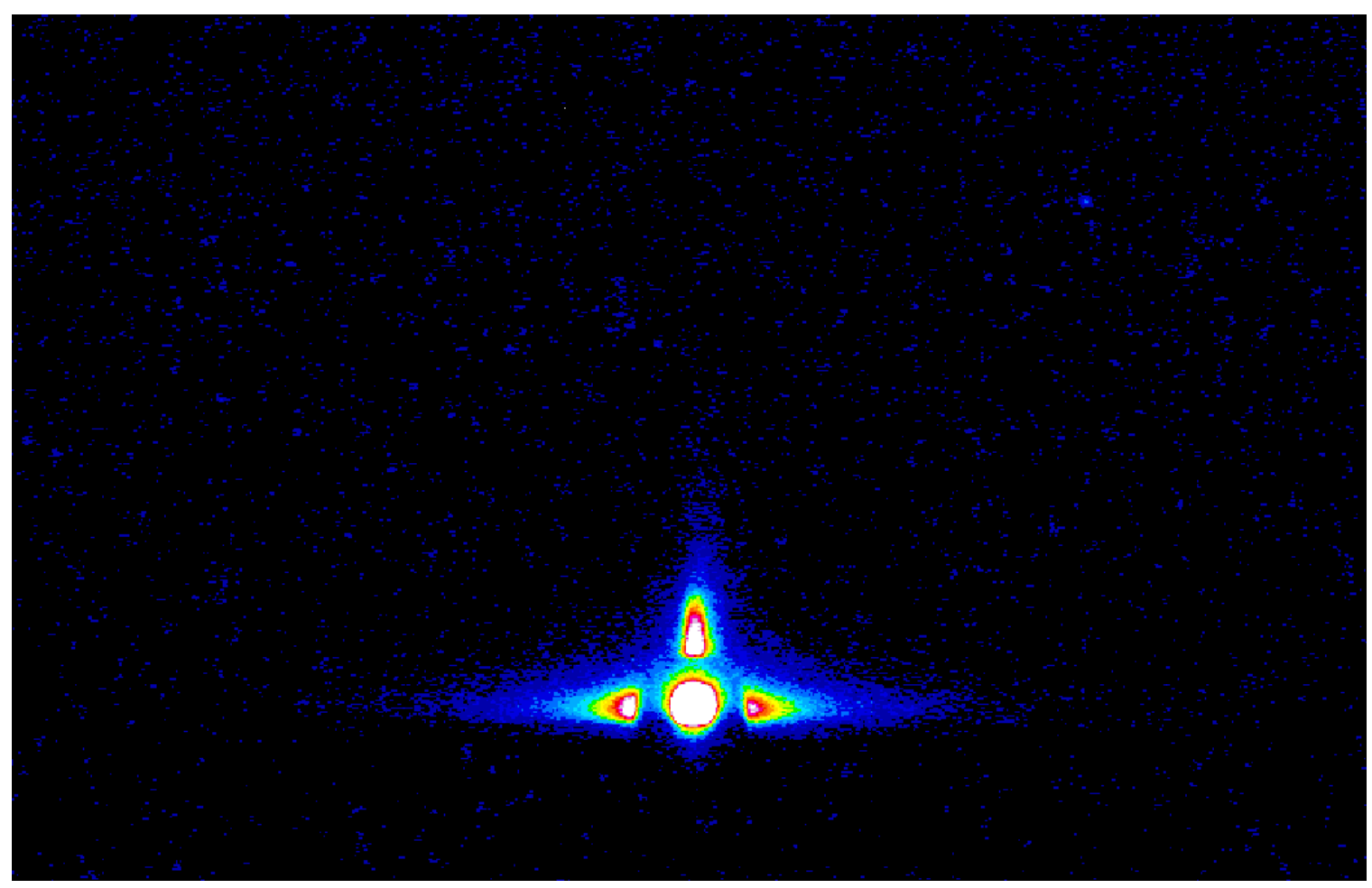

FIgURE 4.14: GISAXS data taken on a blank $\mathrm{SiO}_{2}$ substrate in an identical scenario to Figure 4.13. The lack of diffusely scattered energy implies the material studied has a homogenous electron density. The signal detected just outside the beam blocker is created by smearing of the incident x-ray beam in the alignment steps. These artifacts can be subtracted in data analysis, or more simply can be avoided by analyzing data at offset angles.

imaging plates. Unlike the CCD detector, data on the imaging plates was not able to be read in real time, however the imaging plates have a much larger saturation threshold, allowing for a more statistically significant amount of data to be collected. Each data set used in this study was gathered over a four hour period.

Figure 4.12 shows the geometry of the incident x-ray beam irradiating the sample then scattering into the detector. Figure 4.13 displays raw data collected from an aerogel film by an imaging plate which can be contrasted with Figure 4.14 displaying data collected on a blank $\mathrm{SiO}_{2}$ substrate. Both images show the direct beam path (which is being blocked but still significantly shows up on the imaging plate). On the blank substrate, smearing lines which are artifacts from beam alignment are apparent. The diffuse scattering which illuminates Figure 4.13 contains the information sought about the porous material system. A pie slice shaped cut beginning at the beam origin 


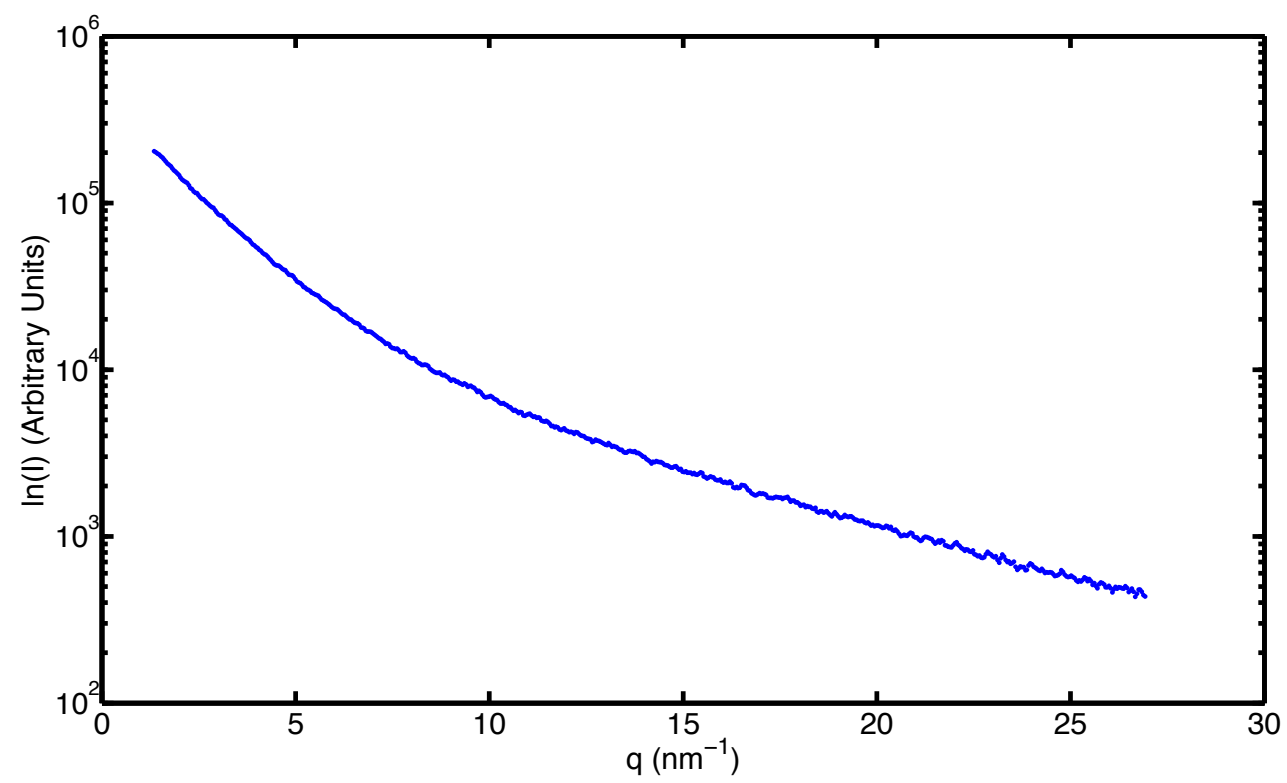

FiguRE 4.15: An intensity profile of GISAXS data from the beam center toward the edge of the detector.

and extending until only background noise is detectable is isolated and integrated over to form the $I$ vs $q$ data shown in Figure 4.15. To avoid contaminating the data with artifacts from the smearing of the main beam, the integration region is offset by $45^{\circ}$.

Analyzing SAXS data can be cumbersome and ambiguous if one is not careful. With so much information contained within a dataset, it is easy to attempt to either overfit information or assign information to a feature incorrectly.[139-141, 145] However, as previously stated, there is a long history of using SAS to analyze porous silica composites and particularly xerogels and aerogels.[97] And while the unique geometry of GISAXS increases the difficulty of collecting quality data, analyzing this data is virtually identical to that of frequently studied aerogel monoliths.[117, 142, 146] Furthermore, for the scope of this work, the more nuanced crossover regimes within the data will be ignored. Focus will be given to two limiting scenarios: the Guinier limit and the Porod limit. The radius of gyration, $r_{g}$, can be determined from

$$
I(q)=I(0) \exp \left(-q^{2} r_{g}^{2} / 3\right)
$$




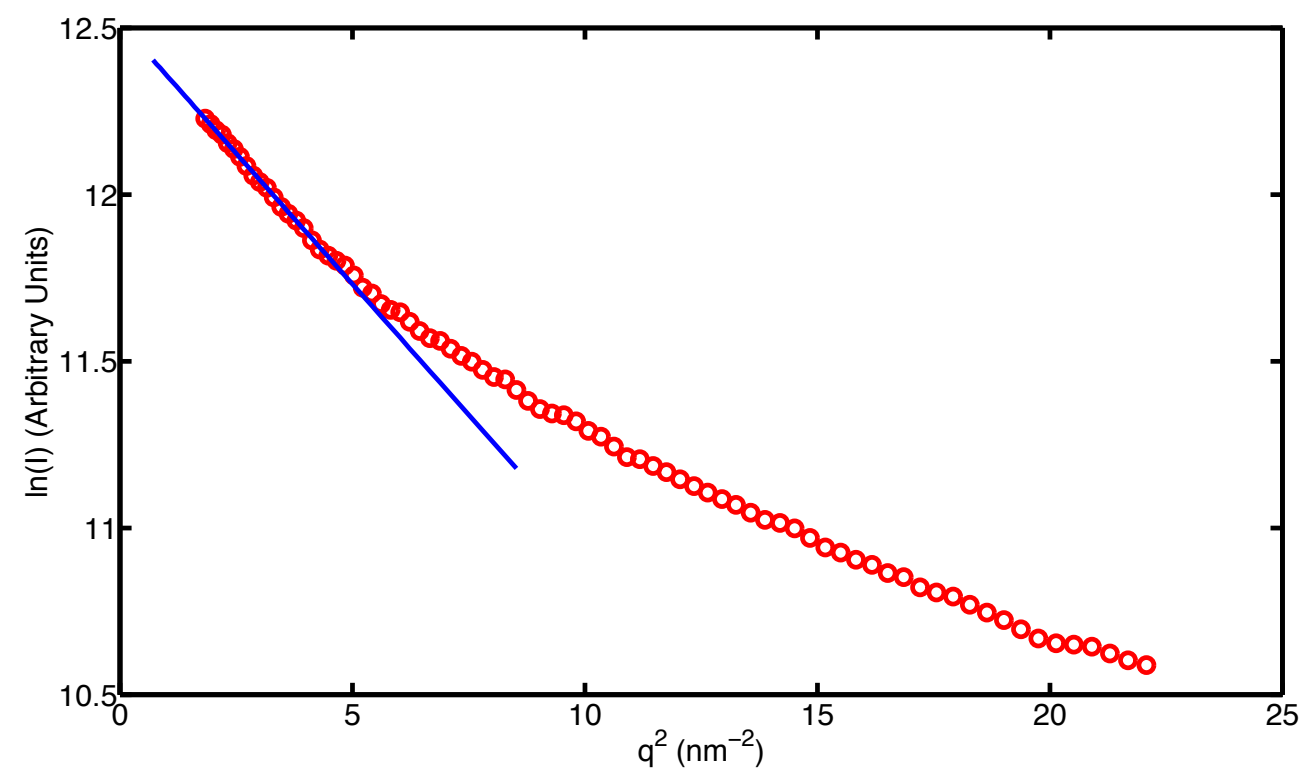

FigURE 4.16: GISAXS data converted to a Guinier plot at low angles to isolate the pores' average radius of gyration.

in the limit that $q \times r_{g}<1.3$.[140,141]. Figure 4.16 displays the low angle data from the previous figure plotted as a Guinier plot (I vs $\mathrm{q}^{2}$ ). From the slope, $m$, of the low angle linear regime of this plot, the radius of gyration can be determined: $r_{g}=\sqrt{-3 m}$. If the feature is spherical (or globular) then the diameter is $d=r_{g} 10 / 3$.

In the Porod limit, as $q$ approaches infinity, for non fractal two-phase systems, Porod's law states that $I \propto q^{-n}$ where $n=4$. Variable $n$ is referred to as the Porod exponent. However for fractal structures, the Porod exponent can be used to determined the fractal nature of the system. If $1<n<3$ then the system contains mass fractal features (sometimes called volume fractals) and the mass fractal dimension, $D_{m}$ is equal to $n$. Generally $D_{m}$ is not observed to be significantly less than 2. Values of $D_{m}$ near 2 indicate a greater degree of fractal cross linking in the system with random orientation and bonding existing between the chains. As $D_{m}$ approaches 3 the structure begins to resemble that of a clustered network. Alternatively if $3<n<4$ then the system exhibits surface fractal features and the 


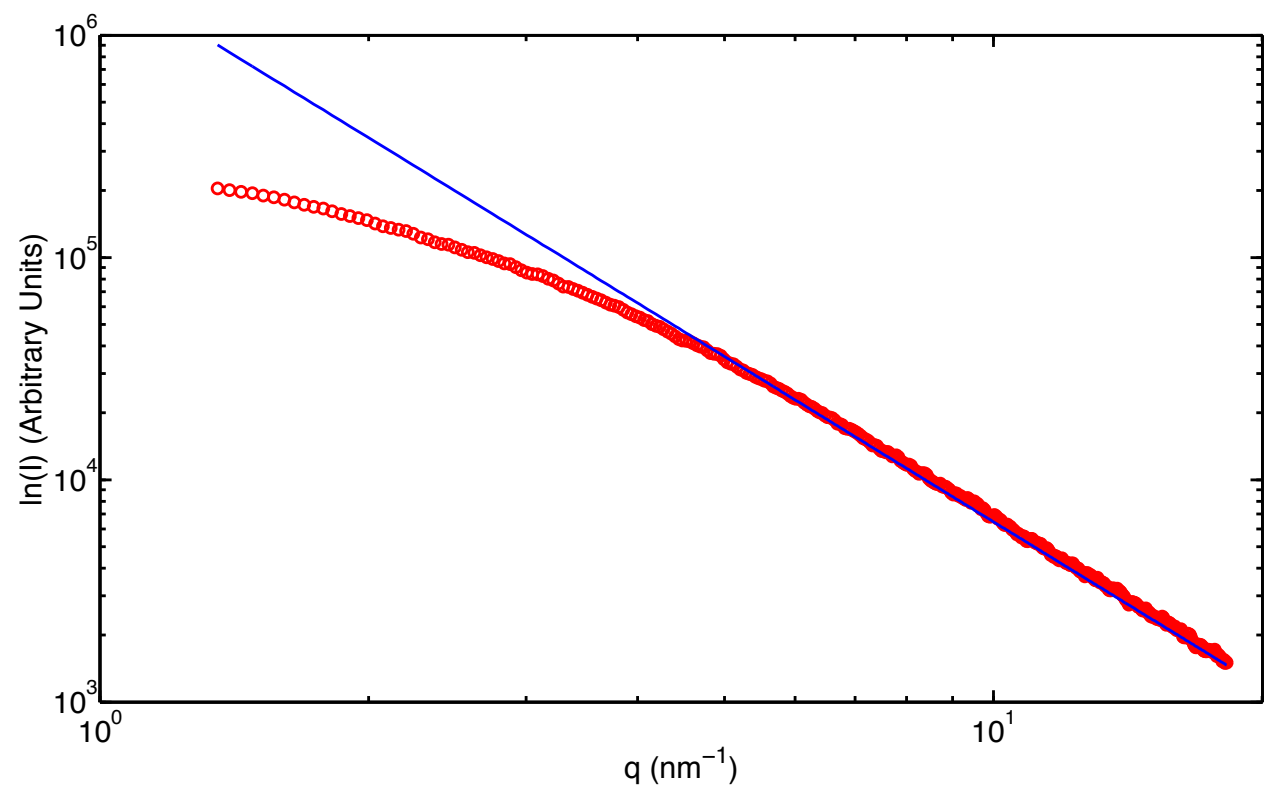

FIGURE 4.17: GISAXS data at large exit angles trending towards a power law decay indicating the Porod limit.

surface fractal dimension is $D_{s}=6-n .[115,147,148]$ Surface fractals with values of $n$ closer to 3 have a more tortious surface but lack the cross links of a mass fractal. As $n$ approaches 4 the shapes are approaching a perfectly smooth surface. To summarize, the Porod exponent, $n$, varies from values near 2, (highly branched and cross linking mass fractals), to values just below 3, (mass fractals with less interlinking) to values just above 3 , (surface fractal with a highly rough surface) to values near 4 (smooth surface). Figure 4.17 displays the exponential decay fit on the data taken from Figure 4.13 and Figure 4.15. Researchers studying aerogels and xerogels have measured decays ranging from $n=1.9$ to $n=4$ with significant variation from mass fractal, to surface fractal, and to planar pores varying based on fabrication conditions. $[145,149]$ While several other parameters can be indirectly determined with a more detailed analysis of the data, for the sake of this study, interpretation is limited to correlating $n$ and $r_{g}$ to fabrication parameters and using these variables to aid in explaining thermal conductivity results.

To understand the measured thermal conductivity results, the sample's porosity 
and thickness also must be determined. To accomplish this, x-ray reflectivity (XRR) is employed. The geometry of an XRR experiment is similar to that of a GISAXS experiment: an incident x-ray beam is focused onto the sample and its response measured by a detector. However, unlike SAXS (and similar to x-ray defraction) it is the specular reflection of the beam that is measured (and the diffuse scattering is ignored). The incident angle of the beam is varied and the detector equivalently varied to acquire data at each angle. From XRR a film's thickness, index of refraction, surface roughness, and interface roughness can be determined.[117, 150] For a twophase system of known materials the index of refraction can be used to determine the percent composition of each phase (and in the case of porous materials, the porosity).

\subsubsection{Results and discussion}

Table 4.2 shows the acid, base, modifying agent, and heat treatment attributes of each sample fabricated for this study. General pyrolization, denoted by $P$ in the HTT column, refers to a $400^{\circ} \mathrm{C} \mathrm{HTT}$ for 1 hour. Variations from this are $3-1$, referring to a $300^{\circ} \mathrm{HTT}$ for 1 hour, and $4-2$ referring to a $400^{\circ} \mathrm{HTT}$ for 2 hours. Note the four samples under the "Heat Treat" label were all made from the exact same batch of solution, spin coated onto 4 substrates and dried simultaneously before heat treatment to allow more direct comparison betweens samples. Sample porosity is obtained from XRR with an an estimated uncertainty of $1 \%$. The average micropore diameter and Porod exponent are obtained from SAXS with uncertainties of less than $2 \%$ in each. The volumetric heat capacity and thermal conductivity of each aerogel film obtained from $3 \omega$ are also displayed with uncertainties of $8 \%$ and $10 \%$ respectively. These values are determined by considering the uncertainty in the thermophysical properties of the substrate and capping layer, film thicknesses, the heating line dimensions, the thermoresistive response of the heating line, and the measurement error in $V_{3 \omega}$. The error bars on data in subsequent figures represent one standard deviation of uncertainty. For clarity, error bars are plotted on a single data point in each plot. 
The scale of the error bars is representative for each measured value. Additionally, the specific heat capacity per unit mass, $c$, is displayed in Table 4.2 and is obtained using the known density of amorphous silica, $2200 \mathrm{~kg} / \mathrm{m}^{3}$, and the aerogel porosity, $P$ :

$$
c=\frac{C}{\rho_{\mathrm{SiO} 2}(1-P)}
$$

The first and perhaps most important attribute to correlate with any aerogel recipe is the porosity of the resulting system. These samples fall in two clear regimes, those with $P<70 \%$ and those with $P>80 \%$. These can be referred to as xerogels and aerogels respectively. Note, the exact definition of a xerogel vs aerogel is somewhat ambiguous and will differ depending on the work being read. The clear jump which can be seen by observing the porosity change in the TMCS series is indicative of the underlying difference between the two names. Generally, xerogels differ from aerogels due to collapsing of a portion of the porous system as the liquid phase evaporates during drying. The data indicates samples $80 \mathrm{~T}, 95 \mathrm{~T}, 110 \mathrm{~T}, 125 \mathrm{~T}$, and $110 \mathrm{H}$ as well as their heat treated counterparts fall into the xerogel regime. The xerogels have a Porod exponent near 3 indicating surface fractals (as opposed to mass fractals) with minimal cross linking within the pores. Within the aerogel regime samples, increasing base concentration correlated to an increase in porosity over nearly the entire range present. It seems in the samples fabricated with the highest base concentration the trend slows or stops. Increased base concentration correlated to smaller pore size and greater cross linking in the mass fractals. The effect was relatively modest. However there is a clear difference when comparing the pore size and fractal dimension of xerogels with aerogels of the same sample series.

In all scenarios heat treatment showed an increase in porosity compared to the non heat treated counterpart. In the aerogel samples heat treatment also caused the samples to increase in thickness by approximately 50\%. Additionally, heat treated samples exhibited a larger average pore diameter and a larger Porod exponent while remaining in the mass fractal regime (implying some decrease in the tortuosity within 
TABle 4.2: List of All Unique Sample Fabrication Conditions and Their Measured Properties.

\begin{tabular}{|c|c|c|c|c|c|c|c|c|c|c|}
\hline \multirow[t]{2}{*}{ Label } & Acid & Base & Mod Ag & $\mathrm{HT}$ & Por & d & $\mathrm{n}$ & $\mathrm{C}$ & $\mathrm{c}$ & $\mathrm{k}$ \\
\hline & & $\mu \mathrm{L}$ & & & $\%$ & $\mathrm{~nm}$ & & $\frac{K J}{m^{3} K}$ & $\frac{J}{k g K}$ & $\frac{W}{m K}$ \\
\hline \multicolumn{11}{|l|}{ TMCS } \\
\hline $80 \mathrm{~T}$ & $\mathrm{HCl}$ & 80 & TMCS & - & 54 & 4.7 & 3.1 & 790 & 780 & 0.392 \\
\hline $95 \mathrm{~T}$ & $\mathrm{HCl}$ & 95 & TMCS & - & 55 & 4.5 & 3.1 & 760 & 770 & 0.381 \\
\hline $110 \mathrm{~T}$ & $\mathrm{HCl}$ & 110 & TMCS & - & 60 & 4.4 & 2.8 & 700 & 790 & 0.328 \\
\hline $125 \mathrm{~T}$ & $\mathrm{HCl}$ & 125 & TMCS & - & 61 & 4.4 & 2.9 & 660 & 770 & 0.309 \\
\hline $140 \mathrm{~T}$ & $\mathrm{HCl}$ & 140 & TMCS & - & 83 & 3.6 & 2.3 & 320 & 850 & 0.082 \\
\hline $165 \mathrm{~T}$ & $\mathrm{HCl}$ & 165 & TMCS & - & 85 & 3.4 & 2.3 & 280 & 850 & 0.075 \\
\hline $185 \mathrm{~T}$ & $\mathrm{HCl}$ & 185 & TMCS & - & 86 & 3.3 & 2.2 & 270 & 870 & 0.064 \\
\hline \multicolumn{11}{|c|}{ TMCS-Pyr } \\
\hline $80 \mathrm{TP}$ & $\mathrm{HCl}$ & 80 & TMCS & $\mathrm{P}$ & 58 & 5.0 & 3.0 & 710 & 770 & 0.363 \\
\hline 110TP & $\mathrm{HCl}$ & 110 & TMCS & $\mathrm{P}$ & 65 & 5.1 & 3.1 & 590 & 760 & 0.281 \\
\hline $140 \mathrm{TP}$ & $\mathrm{HCl}$ & 140 & TMCS & $\mathrm{P}$ & 92 & 4.1 & 2.6 & 150 & 830 & 0.040 \\
\hline $185 \mathrm{TP}$ & $\mathrm{HCl}$ & 185 & TMCS & $\mathrm{P}$ & 95 & 4.0 & 2.6 & 90 & 840 & 0.024 \\
\hline \multicolumn{11}{|l|}{ HMDZ } \\
\hline $110 \mathrm{H}$ & $\mathrm{HCl}$ & 110 & HMDZ & - & 65 & 4.1 & 2.9 & 620 & 800 & 0.229 \\
\hline $140 \mathrm{H}$ & $\mathrm{HCl}$ & 140 & HMDZ & - & 85 & 3.6 & 2.2 & 270 & 830 & 0.054 \\
\hline $165 \mathrm{H}$ & $\mathrm{HCl}$ & 165 & HMDZ & - & 86 & 3.0 & 2.3 & 260 & 840 & 0.049 \\
\hline $185 \mathrm{H}$ & $\mathrm{HCl}$ & 185 & HMDZ & - & 88 & 2.9 & 2.1 & 230 & 880 & 0.040 \\
\hline $200 \mathrm{H}$ & $\mathrm{HCl}$ & 200 & HMDZ & - & 91 & 2.8 & 2.0 & 180 & 900 & 0.029 \\
\hline $220 \mathrm{H}$ & $\mathrm{HCl}$ & 220 & HMDZ & - & 90 & 2.7 & 2.0 & 200 & 890 & 0.032 \\
\hline \multicolumn{11}{|c|}{ HMDZ-Pyr } \\
\hline 110HP & $\mathrm{HCl}$ & 110 & HMDZ & $\mathrm{P}$ & 68 & 4.2 & 3.0 & 560 & 790 & 0.211 \\
\hline 140HP & $\mathrm{HCl}$ & 140 & HMDZ & $\mathrm{P}$ & 91 & 4.2 & 2.6 & 160 & 820 & 0.036 \\
\hline $165 \mathrm{HP}$ & $\mathrm{HCl}$ & 165 & HMDZ & $\mathrm{P}$ & 93 & 3.8 & 2.6 & 130 & 820 & 0.026 \\
\hline $185 \mathrm{HP}$ & $\mathrm{HCl}$ & 185 & HMDZ & $\mathrm{P}$ & 94 & 3.6 & 2.5 & 110 & 850 & 0.021 \\
\hline 200HP & $\mathrm{HCl}$ & 200 & HMDZ & $\mathrm{P}$ & 98 & 3.2 & 2.5 & 40 & 860 & 0.010 \\
\hline $220 \mathrm{HP}$ & $\mathrm{HCl}$ & 220 & HMDZ & $\mathrm{P}$ & 96 & 3.2 & 2.4 & 80 & 860 & 0.015 \\
\hline \multicolumn{11}{|c|}{ Heat Treat } \\
\hline HT0 & $\mathrm{HCl}$ & 185 & HMDZ & - & 88 & 2.9 & 2.2 & 290 & 880 & 0.041 \\
\hline HT300-1 & $\mathrm{HCl}$ & 185 & HMDZ & $3-1$ & 91 & 3.3 & 2.4 & 170 & 860 & 0.032 \\
\hline HT400-1 & $\mathrm{HCl}$ & 185 & HMDZ & $\mathbf{P}$ & 94 & 3.5 & 2.5 & 90 & 830 & 0.022 \\
\hline HT400-2 & $\mathrm{HCl}$ & 185 & HMDZ & $4-2$ & 98 & 4.2 & 2.7 & 70 & 800 & 0.013 \\
\hline \multicolumn{11}{|l|}{$\mathrm{HNO}_{3}$} \\
\hline N185H & $\mathrm{HNO}_{3}$ & 185 & HMDZ & - & 82 & 3.2 & 2.6 & 320 & 810 & 0.091 \\
\hline N185HP & $\mathrm{HNO}_{3}$ & 185 & HMDZ & $\mathrm{P}$ & 85 & 3.3 & 2.8 & 260 & 790 & 0.079 \\
\hline N185H2 & $\mathrm{HNO}_{3}$ & 185 & HMDZ & - & 40 & - & 3.4 & 1000 & 760 & 0.549 \\
\hline N185H2P & $\mathrm{HNO}_{3}$ & 185 & HMDZ & $\mathrm{P}$ & 38 & - & 3.4 & 1020 & 750 & 0.521 \\
\hline $\mathrm{SiO} 2$ & & & & & 0 & & & 1700 & 745 & 1.3 \\
\hline
\end{tabular}


the pores). It can be inferred that pyrolization is causing some bonds to break after the highly porous network has been set. This relieves internal stress and allows the pores to relax and spring back to a larger diameter. The four samples in the series labeled "Heat Treat" help confirm this result. A trend of increasing porosity, pore diameter, and Porod exponent exists, ranging from the non heat treated sample (HTO) to the more moderately heat treated samples (HT300-1 and HT400-1) and through to the most heat treated sample (HT400-2). These results are in agreement with previous heat treatment studies on aerogel fractal dimension[116] which indicate a strong correlation with $\mathrm{HTT}$ up to $500^{\circ} \mathrm{C}$ at which point the fractal nature of the pores vanishes.

In general, for a given base concentration, the HMDZ modified aerogels were more porous, exhibited smaller pore diameters (around a $0.5 \mathrm{~nm}$ difference) and a lower Porod exponent (near a 0.2 to 0.4 difference) compared to their TMCS counterparts. The modifying agent alters the molecule capping the terminal ends of the pores. The size and charge of these capping molecules is likely altering the diameter of the pores. Ruffner et al. also identified noticeable difference between HMDZ and TMCS modified aerogel films. In their study HMDZ modified samples had increased surface roughness, increased film thickness, higher porosities and greater uniformity across the entire sample compared to the TMCS counterparts.[100]

Lastly, four samples were generated by replacing the acid catalyst with $\mathrm{HNO}_{3}$. Two batches of sol-gel were created. Sample N185H2 had double the acid concentration of $\mathrm{N} 185 \mathrm{H}$. These are unique recipes attempted by the Soft Materials Laboratory. A pyrolized version of each sample was also created. In the case of N185H2, it seems that a full collapse of the gel structure occurred. It should be emphasized that the earlier mentioned xerogels only exhibited a partial collapse of the structure and a continuous, chemically bonded structure with distinct features still existed. All duplicates of the N185H2 sample created were incredibly flakey, with powder easily falling off the samples. The values determined are still reported. 
No Guinnier region was apparent in the SAXS data. The Porod measured for this sample exponent was the greatest of any sample measured in this study, 3.4. The more dilute $\mathrm{HNO}_{3}$ sample (N185H and N185HP) successfully formed aerogels of worse quality than the HCL samples. More investigation into this aerogel formulation is needed.

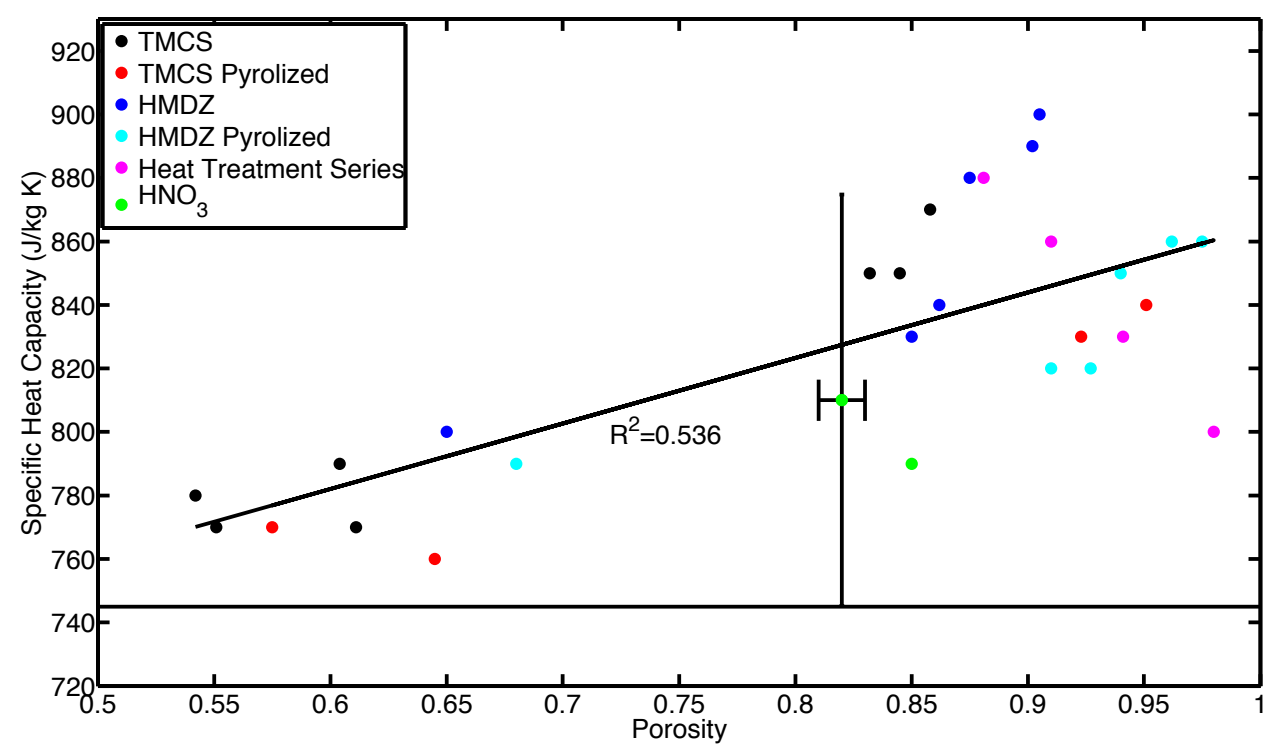

FIGURE 4.18: Measured specific heat capacity vs porosity of xerogel and aerogel films. Error bar shown is representative for all samples. Sold line shows the specific heat capacity of of amorphous $\mathrm{SiO}_{2}$.

One of the first striking thermophysical results from the $3 \omega$ data is that every sample tested has a specific heat capacity greater than fully dense amorphous $\mathrm{SiO}_{2}$. In most of the higher porosity aerogels, this difference is greater than the measurement uncertainty. To investigate the origin of the increase, the trend of specific heat increase is compared to the three structural parameters: porosity, pore diameter, and the fractal dimension of each sample. Figure 4.18 shows a plot of porosity vs specific heat capacity with a moderate coefficient of determination: 0.536. This indicates there is a reasonable amount of correlation between porosity and specific heat capacity of the samples tested. However, correlation does not mean causation. All other factors 
being constant, it seems unlikely that changing sample density alone would alter an intrinsic property like specific heat capacity. It is apparent in this plot that some sample series trended away from the regression fit. The HMDZ series clearly deviated to a higher specific heat capacity than the regression fit while the "Heat Treat" series also trends nearly perpendicular to the regression line.

The following two plots compare the heat capacity to features within the nanostructure of the sample. Figure 4.19 displays the the heat capacity trend compared to the measured pore diameter and Figure 4.20 compares the specific heat capacity to the Porod exponent. The two plots have coefficients of determination of 0.754 and 0.883 respectively. The close values are expected. Across all samples tested, generally the fractal dimension and pore diameter were linked. A greater degree of cross linkage (implying a more mass fractal shape) pulls the pore walls more tightly together, decreasing their diameter. The strong correlation between the microstructure features of the samples and the increasing specific heat capacity warrants further consideration.

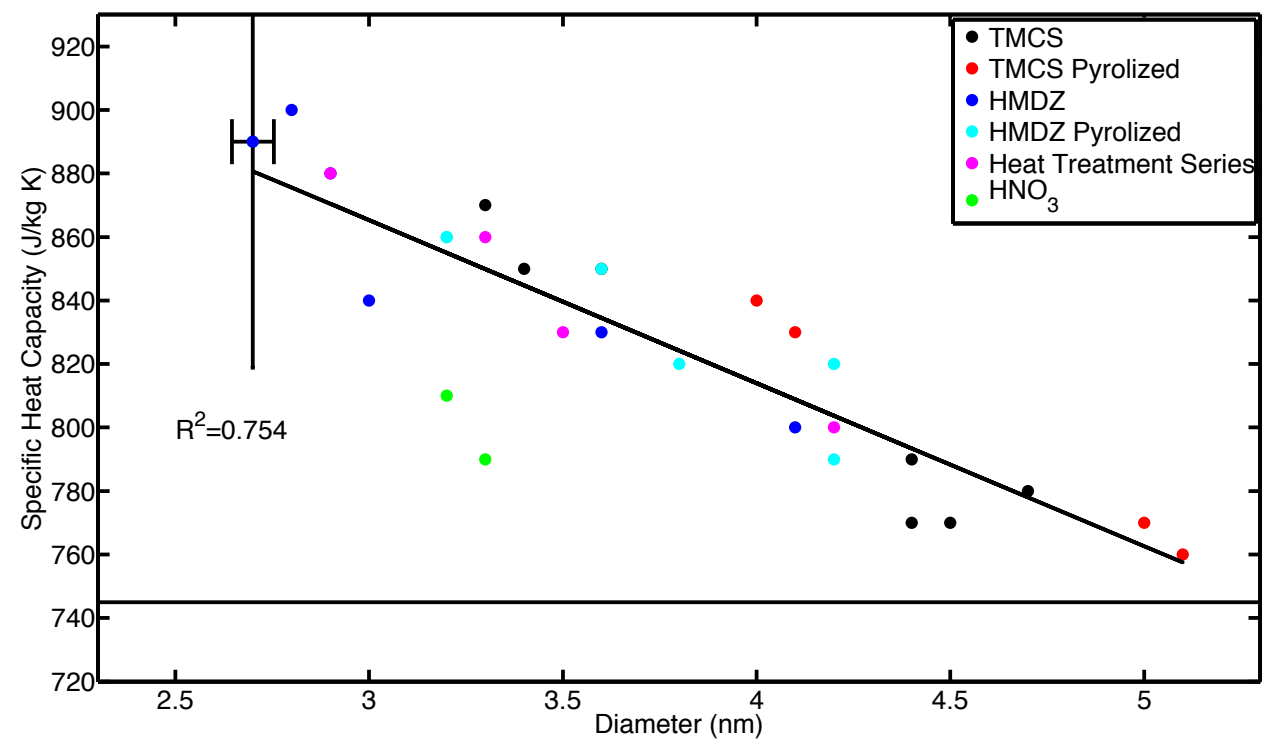

FIGURE 4.19: Measured specific heat capacity vs pore diameter of xerogel and aerogel films. 
Previous work has investigated the fractal network altering the thermophysical properties of silica aerogels and other composite systems. Alexander and Orbach coined the term fracton: the quantized vibrational states on a fractal.[31] Alexander et al. used the concept of a fracton to aid in describing thermal energy storage and transport in glassy materials.[32] Later studies identified phonon-fracton crossover regimes and found unique contributions to the density of states of fractal materials, particularly silica aerogel monoliths. [33, 151-153] However, the regimes in which these effects are dominate are shown to be at ultra low temperatures.[123, 154-156] In studying the transport of thermal energy by fractons, research has shown the existence of a specific dispersion curve and density of states with unique features compared to the traditional phononic dispersion curve. It should be noted that while fractal theory was initially suggested as a general description of energy transport in glassy amorphous materials, it is more commonly accepted that a crossover exists between phonon, fracton, and uncoupled oscillation. These uncoupled oscillations are thought to best describe thermal transport in fully dense amorphous material.[28]

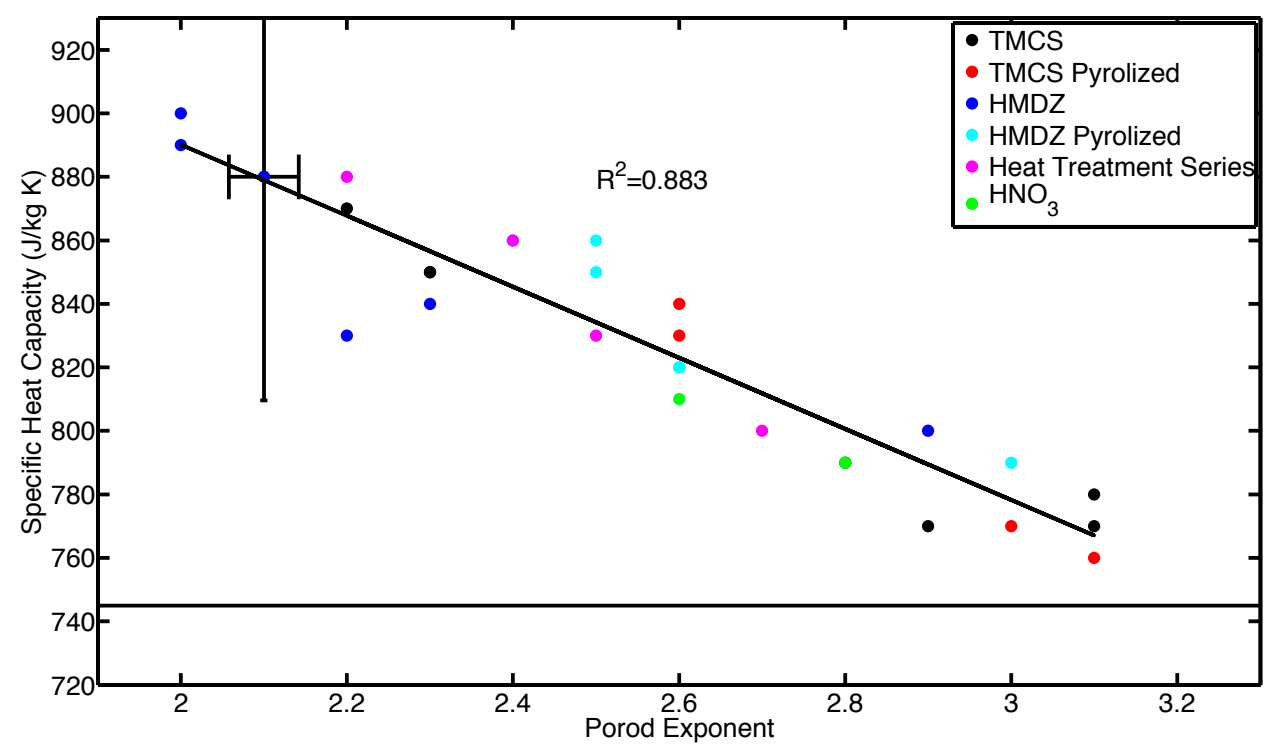

Figure 4.20: Measured specific heat capacity vs Porod exponent of xerogel and aerogel films. 
Scheuerpflug et al. showed that at temperatures below $10 \mathrm{~K}$, silica aerogels have a drastic increase in specific heat capacity compared to fully dense silica with a unique temperature dependence, which confirms studies by previous researchers.[124] At higher temperatures (measured up to $330 \mathrm{~K}$ ) the silica aerogels' specific heat capacity followed the same temperature trend as silica. However each specific heat value measured was consistently larger than amorphous $\mathrm{SiO}_{2}$ with one sample showing an increase around $30 \%$ at all elevated temperatures. These measurements aid to confirm the results shown here. Silica aerogels consistently demonstrate an increased specific heat capacity at room temperatures. With no further structural characterization to consider, Scheuerpflug et al. suggested that the large specific surface area and the additional modes in the dangling bonds of the network were the cause.

Without direct measurements of the vibrational properties of the samples studied in this work, connections between the fractal nature in the samples and the jump in specific heat are left to be inferred. A relatively strong correlation has been shown between the fractal dimension and increase in specific heat capacity of the samples. Furthermore, due to the large range of samples fabricated, nuanced tuning of the fractal nature independent of resulting porosity is apparent in the data set. For example, samples $200 \mathrm{H}$ and $220 \mathrm{H}$ have mass fractal dimensions of 2.0 , porosities of $91 \%$ and $90 \%$, pore diameters of $3.2 \mathrm{~nm}$, and specific heat capacities of $900 \mathrm{~kJ} / \mathrm{kg} \mathrm{K}$ and $890 \mathrm{~kJ} / \mathrm{kg} \mathrm{K}$. On the other hand samples 200HP and 220HP (the heat treated counterparts) achieved greater porosities while having larger Porod exponents and larger pores but still had a lower specific heat capacity $(860 \mathrm{~kJ} / \mathrm{kg} \mathrm{K})$. Additionally the four samples in the heat treatment series showed a trend of increasing fractal dimension and decreasing specific heat capacity despite the porosity of the samples increasing. The larger point illustrated by Figure 4.19 and Figure 4.20 is that there is at least correlation in the samples between the increasing specific heat capacity and decreasing fractal dimension as well as pore diameter. Previous works have illustrated 
temperature independent fracton density of states at angular frequencies below $10^{12}$ $\mathrm{Hz}$ in highly porous aerogels.[151] While the contributions of fractons may not be dominate at elevated temperatures, it seems reasonable to infer the fractal nature of the aerogel microporous system may still play a role in storing thermal energy. Finally, it should be noted that measurements of the specific heat capacity of porous silicon by Wolf and Brendel did not show any deviation from bulk silicon.[157]

Turning to the thermal conductivity data, it is apparent that higher porosity correlates with a lower thermal conductivity. Figure 4.21 clearly depicts this trend. The aerogel thermal conductivity data is plotted over a differential effective medium (DEM) theory prediction which gives an approximation of thermal conductivity in porous materials as a function of its porosity $(P)$ and bulk thermal conductivity $\left(k_{B}\right)$, and serves as a starting point for comparison between samples: [158]

$$
k_{e}=k_{B}(1-P)^{3 / 2}
$$

This equation holds up well for silica materials in general, and has been shown to be very reasonable in predicting the thermal conductivity of aerogel monoliths.[159, 160] This broad porosity based trend line does a reasonable job of anticipating each sample's thermal conductivity. Many of the samples have measured thermal conductivities within experimental uncertainty of Equation 4.3. The non heat treated HMDZ series compares very closely to measurements made by Hopkins et al. on highly similar samples with a measured thermal conductivity of $0.053 \mathrm{~W} / \mathrm{m} \mathrm{K.[161]} \mathrm{However,} \mathrm{our} \mathrm{heat} \mathrm{treated} \mathrm{samples} \mathrm{showed} \mathrm{an} \mathrm{increase} \mathrm{in}$ porosity and decrease in thermal conductivity, which is the opposite result found by Hopkins et al. This is likely due to our more moderate heat treatment temperatures, as others have shown elevated HTT can cause a break down of the fractal network. It is very likely that the trend in fractal dimension of the Hheat treatment series is showing the beginning of this effect. 
As previously stated, unique fabrication processes produced aerogels of differing porosity, which may be attributed to effects of the microstructure altering the total structure of the aerogel. The HMDZ samples, which showed a distinct reduction in thermal conductivity compared to the TMCS series, had a lower Porod exponent and smaller pores. These features may play an indirect role in obtaining ultra high porosity (and therefore ultra low thermal conductivity) TFSA. The more interconnected nature of the HMDZ aerogels may lead to a more robust structure which minimizes harm to the silica network during ambient drying of the thin films. If this results in superior mesoporous level chains of silica remaining connected, then superior thermal insulating aerogels can be fabricated. Within the HMDZ series (as well as the TMCS series) at generally higher base concentrations the pore diameter was minimized seemingly optimizing this effect. Conversely, an alternative route to increasing the porosity is to seemingly break the silica bonds through heat treatment, allowing internal stress to be relieved. This causes the system to expand. Since the amount of silica in the system remains constant, the porosity over the entire thickness of the film increases.

Does the microstructure of the sample directly alter the sample's thermal conductivity? Figure 4.22 compares the deviation of the aerogel samples (porosity greater than 80\%) from Equation 4.3 with each sample's fractal dimension. A moderate correlation seems to exist with a coefficient of determination equal to 0.5255. However the experimental uncertainty in the thermal conductivity measurements (determined to be 10\%) implies that this potential trend is within the error of measurements, or on the edge of measurement uncertainty. Across all samples, pyrolization moved the samples up this trend line (both higher Porod exponent and a thermal conductivity above the predicted value). Within other individual sample series, change in fractal dimension does not correlate with the deviation from Equation 4.3. From this it cannot be definitively concluded that tuning the microstructure is directly altering heat transport phenomena. The most 


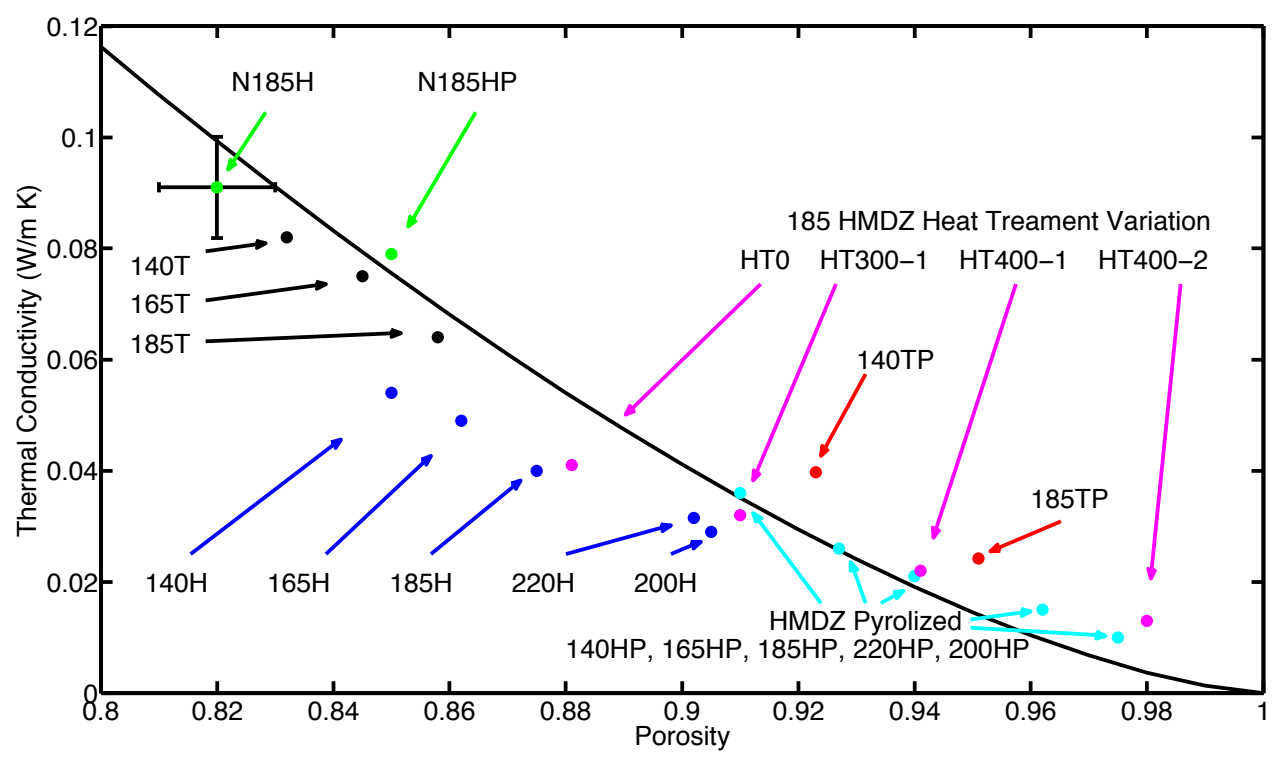

Figure 4.21: Measured thermal conductivity vs porosity of aerogel films. Also plotted is the thermal conductivity predicted from differential effective medium theory.

apparent direct trend in Figure 4.22, heat treatment of samples, may not necessarily be due to structural changes but rather chemical changes within the samples. For amorphous systems in which the mean free path is on the same order as a few interatomic spacings, such a phenomena would be difficult to show at room temperatures. Perhaps aerogels with a high degree of crystallinity would be better candidates to directly observe tuning of the microstructure altering thermal transport.[162]

In summary, as shown to be true in monolithic silica aerogel samples, the thermal conductivity of TFSA is strongly correlated with the sample's porosity. By altering the fractal network's properties during fabrication, the underlying structure of the aerogel can be directly changed, which indirectly alters the system's thermal conductivity. TFSA with porosities as high as $98 \%( \pm 1 \%)$ are shown to have thermal conductivity values as low as $0.010 \mathrm{~W} / \mathrm{m} \mathrm{K}( \pm 0.001 \mathrm{~W} / \mathrm{m} \mathrm{K})$ which expanded the achievable range of thermal conductivities in thin film aerogels. Achieving such high porosities requires a strongly mass fractal system entangling 
the silicon chains and minimizing the diameter of the micropores. Therefore, the entanglement of the microstructure plays an indirect role in minimizing thermal transport in the system. Furthermore, such fractal properties seem to be causing an increase in the specific heat capacity of the aerogels. It is difficult to demonstrate the microstructure of the silica chains directly altering heat transfer mechanisms contributing to thermal transport in this amorphous system at room temperature. While other research shows the effect of fractons at ultra low temperatures, they seem to play a relatively tiny role relative (if any) in transporting thermal energy at room temperature in amorphous fractals such as silica aerogels.

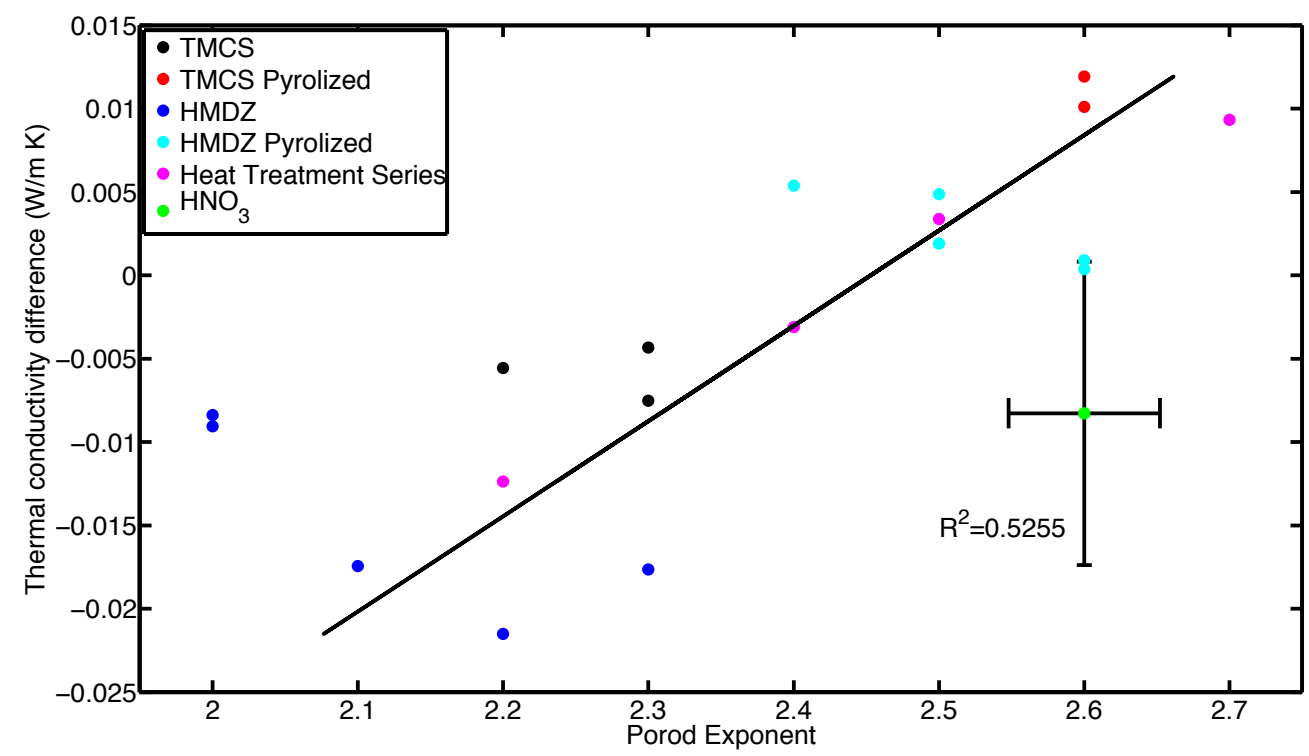

Figure 4.22: Measured thermal conductivity deviation from Equation 4.3 plotted against the Porod exponent for the more mass fractal aerogels. 


\section{Chapter 5}

\section{Pressure Dependent Thermal \\ Transport in Carbon Nanofiber Networks}

Much of the content in the following chapter is reproduced for the manuscript currently under review for publication: "Thermal conductivity of turbostratic carbon nanofiber networks" by M. L. Bauer, C. B. Saltonstall, Z. C. Leseman, T. E. Beechem, P. E. Hopkins, and P. M. Norris.

\section{$5.1 \quad$ Background}

Allotropes of carbon, often forming unique microstructures, have shown a tremendous range of achievable mechanical and thermal properties. Amorphous carbon films can have a thermal conductivity ranging from 0.2 to $2 \mathrm{~W} / \mathrm{m} \mathrm{K}$.[71] On the other hand, crystalline $\mathrm{sp}^{3}$ bonded diamond has measured room temperature conductivities exceeding 1,000 W/m K.[163] Graphite has strong in-plane sp ${ }^{2}$ bonding producing uniquely high thermal conductivities, particularly in its single plane form, graphene.[164, 165] However graphene layers forming graphite are 
connected by weak van der Waals forces resulting in a cross-plane thermal conductivity under $10 \mathrm{~W} / \mathrm{m} \mathrm{K}$.[166] Porous carbon nano foams with a high degree of compliance have measured thermal conductivities ranging from 0.05 to $180 \mathrm{~W} / \mathrm{m}$ K.[167] Carbon fibers, a material that has been shown to span the range of thermal conductivites from under $1 \mathrm{~W} / \mathrm{m} \mathrm{K}$ to over $1000 \mathrm{~W} / \mathrm{m} \mathrm{K}$, are the focus of this study.[168]

Carbon fibers are rod shaped structures of $\mathrm{sp}^{2}$ bonded carbon with diameters ranging from tens of microns to under a hundred nanometers (often called nanofibers). The thermal conductivity of an individual fiber is strongly correlated to phonon coherence length which is a function of the crystallite diameter. The size of the crystalline grains within the fiber can be tuned through heat treatment.[169-171] In the graphitic limit, an individual carbon fiber behaves much like a carbon nanotube or graphene, with room temperature thermal conductivities as high as $1400 \mathrm{~W} / \mathrm{m} \mathrm{K.[172]} \mathrm{Conversely,} \mathrm{in} \mathrm{the} \mathrm{turbostratic} \mathrm{limit,} \mathrm{in} \mathrm{which} \mathrm{small}$ grain sizes limit phonon transport, thermal conductivity of the individual fibers approaches that of cross-plane graphene, with reported values less than $20 \mathrm{~W} / \mathrm{m}$ K.[171] Additionally, the intrinsic thermal conductivity of carbon fibers can be influenced by nanovoids within the fibers.[173, 174] Growth conditions dictate structural parameters and therefore thermal transport properties.

The term carbon fiber is applied to a broad range of fibers with very diverse properties. In particular the maximum heat treatment temperature (HTT) of the material dictates the underlying properties. Historically, carbon fibers have been divided into $\mathrm{HTT}$ categories of $1000^{\circ} \mathrm{C}$ to $1500^{\circ} \mathrm{C}$ called carbon fibers, those of $\mathrm{HTT}$ between $1500^{\circ} \mathrm{C}$ and $2000^{\circ} \mathrm{C}$ called carbonized fibers, and those with HTT above $2000^{\circ} \mathrm{C}$ called graphitized fibers. However more recently the term "high strength" is used to refer to low HTT fibers and "high modulus" used to refer to high HTT fibers and the term "carbon fibers" has become a more general term encompassing all HTT.[175] As the names imply, there is a general trade off between maximizing 
Young's modulus or maximizing the tensile strength of a fiber. Beyond HTT, a carbon fiber's properties are influenced by the organic fiber precursor. These controls allows carbon fibers' mechanical properties to be optimized for desired applications. Some examples include reinforcing materials such as sporting equipment, cement, and in aerospace applications.[176] Since carbon fibers can have thermal conductivities much higher than common metals, they could serve in thermal management applications such as aiding in heat dissipation in brake disks. Alternatively, the low thermal conductivity achievable in some forms of carbon fibers is ideal for thermal insulation such as insulating the US Space Shuttle Orbiter's leading edges.[176]

Thermal management applications utilizing carbon nanofibers require a composite material containing a matrix of fibers with an epoxy or a fluid filling the voids.[177] Such a composite can be tuned to meet the thermal and mechanical requirements of the application. However, it becomes more difficult to anticipate the total thermal transport as the system increases in complexity. The system geometry, interaction between materials, and deviation of each material from bulk, monolithic thermal properties influence the effective thermal conductivity of the system. Each of these terms must be accounted for to confidently predict how such a system will respond in a thermal management application. In the carbon fiber networks studied, these parameters are systematically varied to isolate the factors contributing the effective thermal transport of the system. To gain general insight into the mechanisms dictating the composite material's rate of heat flux, a series of thermal conductivity tests are performed with initial fiber fill fraction, heat treatment temperature (HTT), loading pressure, and gas pressure varied. The bidirectional modification of $3 \omega$ described in Chapter 3 is ideally suited to characterize carbon fiber networks under varying compressive pressure. By observing the effects of fiber crystal size, fiber network density, particle orientation, fluid conductivity, and conductance via fiber to fiber contacts on the measured thermal conductivity, a link can be formed between the structural characteristics of 
the system and the resulting thermophysical properties. By generally considering the interdependent contributions of these features to thermal transport under the varied set of testing conditions, confidence is given to applying predictive thermal modeling to a broader range of fiber composite materials (varying the fluid material, type of fiber, fiber density, etc.) and the conditions in which they operate (such as straining the system). Furthermore, key considerations when designing fibrous thermal management solutions can be isolated.

\subsection{Sample preparation}

The carbon nanofiber networks used in this work were synthesized using a chemical vapor deposition process. Palladium catalyst nanoparticles were initially heated in a furnace with an inert gas environment. Upon reaching $550^{\circ} \mathrm{C}$ an ethylene and oxygen mixture was passed over the Pd nanoparticles. Varying gas flow time alters the densities of the synthesized samples.[178] This fabrication method has been shown to create fibers with little atomic order.[179] Samples were fabricated by Professor Zayd Leseman of the University of New Mexico.

Five unique samples were fabricated, labeled S1, S2, S3, S4, and S5 with respective densities of $90,110,130,160$, and $200 \mathrm{~kg} / \mathrm{m}^{3}$. Density measurements were taken with a vernier caliper and a precision weighing balance. Measurement error is estimated to be $\pm 10 \mathrm{~kg} / \mathrm{m}^{3}$. By assuming an individual fiber density of $1700 \mathrm{~kg} / \mathrm{m}^{3}$ as has been measured on similar fibers,[171] we infer an as grown fill fraction, $\phi_{0}$, ranging from $5 \%$ (on S1) to $12 \%$ (on S5). The least dense sample, S1, was divided into five parts. Four of these portions were heat treated at maximum $\mathrm{HTT}$ of $900^{\circ} \mathrm{C}, 1100^{\circ} \mathrm{C}, 1300^{\circ} \mathrm{C}$, and $1500^{\circ} \mathrm{C},\left(\mathrm{S}_{09}, \mathrm{~S}_{11}, \mathrm{~S}_{13}\right.$, and $\left.\mathrm{S} 1_{15}\right)$ respectively. The last portion underwent no heat treatment $\left(\mathrm{S}_{00}\right)$. Each heat treatment process took place in an inert argon environment with zirconium foil used as a getter to capture any remaining oxygen in the system. The temperature of the apparatus was ramped at a rate of $120^{\circ} \mathrm{C}$ per hour 
until reaching the prescribed temperature. The samples were held at the respective maximum HTT for 2 hours before cooling at the same rate they were heated. Each density measurement after heat treatment varied by no more than $5 \mathrm{~kg} / \mathrm{m}^{3}$ from the original measurement. This is within the measurement uncertainty, so we infer the composite underwent no significant density change. The heat treatment furnace utilized for this work was provided by Professor Elizabeth J. Opila of the University of Virginia.

\subsection{Fiber characterization}

Prior to investigating the thermal transport in the carbon fiber network, it is necessary to understand the nature of the individual carbon fibers within the composite. The atomic structure of the carbon fibers dictates their intrinsic thermal conductivity, $k_{f}$, which influences the effective thermal conductivity of the system, $k_{e}$. The density of crystal boundaries, as well as the size and density of voids within the fibers will determine the frequency at which thermal energy carriers scatter. A material's thermal conductivity is a function of the thermal energy carrier's scattering rate. The crystal size is observed via Raman spectroscopy. The Raman spectroscopy data used for this work was acquired by Christopher B. Salstonall. He and Thomas E. Beechem were instrumental in interpreting the data in this section.

Our Raman spectra were collected using a Renishaw InVia Raman microscope. The system utilizes the $488 \mathrm{~nm}$ line of an Ar ion laser in $180^{\circ}$ back scattering geometry and a $3000 \mathrm{~g} / \mathrm{mm}$ grating. The sample was irradiated with $0.1 \mathrm{~mW}$ laser power through a 50x 0.75 NA objective yielding a spot diameter of $900 \mathrm{~nm}$. Ten spectra were collected, 360 second acquisition times, on each sample at 10 different locations of the sample spaced $10 \mu \mathrm{m}$ apart. The main features of the carbon Raman spectra used for our analysis are the two peaks at $\sim 1350$ and $\sim 1600 \mathrm{~cm}^{-1}$. The peak located at $1600 \mathrm{~cm}^{-1}$ corresponds to $\mathrm{sp}^{2}$ graphite-like $\Gamma$ point vibrations and is thus named 
the $\mathrm{G}$ or graphite peak. The lower frequency peak originates from modes at the $K$ point of the Brillioun zone of graphite which in a perfect graphite crystal are disallowed Raman active modes. However, disorder from the turbostratic nature of these fibers relaxes the selection rules giving rise to this observed D peak at 1350 $\mathrm{cm}^{-1}$. Naturally, this leads to the relative intensities of the G and D peaks being used to quantify disorder in carbon systems. More over, this ratio of intensities can be used to estimate the size of the crystalline regions known as the cluster diameter or in-plane correlation length, $L_{a}$, which is an indicator of the amount of disorder. $[180,181]$

Where the ratio of the $\mathrm{D}$ to $\mathrm{G}$ peak Raman intensities $(I(\mathrm{D}) / I(\mathrm{G}))$ provides insight into the microstructure of the carbon nanofibers, additional aspects of the Raman spectra, such as the peak positions and widths of the peaks, provide additional information to characterize our samples. If one considers the graphene dispersion, see Figure 2(a) of Ref. [181], only the modes at the $\Gamma$ point ( $G$ peak) are Raman active in pure graphite. However, as disorder is introduced the selection rules are relaxed allowing for a larger range of modes away from the $\Gamma$ point to be Raman active following $\Delta k \approx \pi / L_{a}$, where $k$ is the phonon wave vector. This effect is also seen in the $K$ point modes (D peak). This changes the observed Raman spectra in two ways; broadening and shifting the peaks. In the case of the G peak we can see that as $\Delta k$ increases the average frequency also increases leading to an observed increase in the peak position. Since the scattering phonons are now of a non-singular frequency range, the peak broadens. The same disorder broadening will be observed in the case of the D peak, but the peak position shift is more complicated. This assessment assumes that we are starting at perfect graphite and slowly introducing disorder until we have nanocrystalline graphite, Stage 1 of Ferrari and Robertson's 3 Stage amorphization model.[181]

To extract the peak intensities, each spectrum was fitted with a two function fit following the procedure outlined by Ferrari and Robertson,[181] a Voigt for the D 


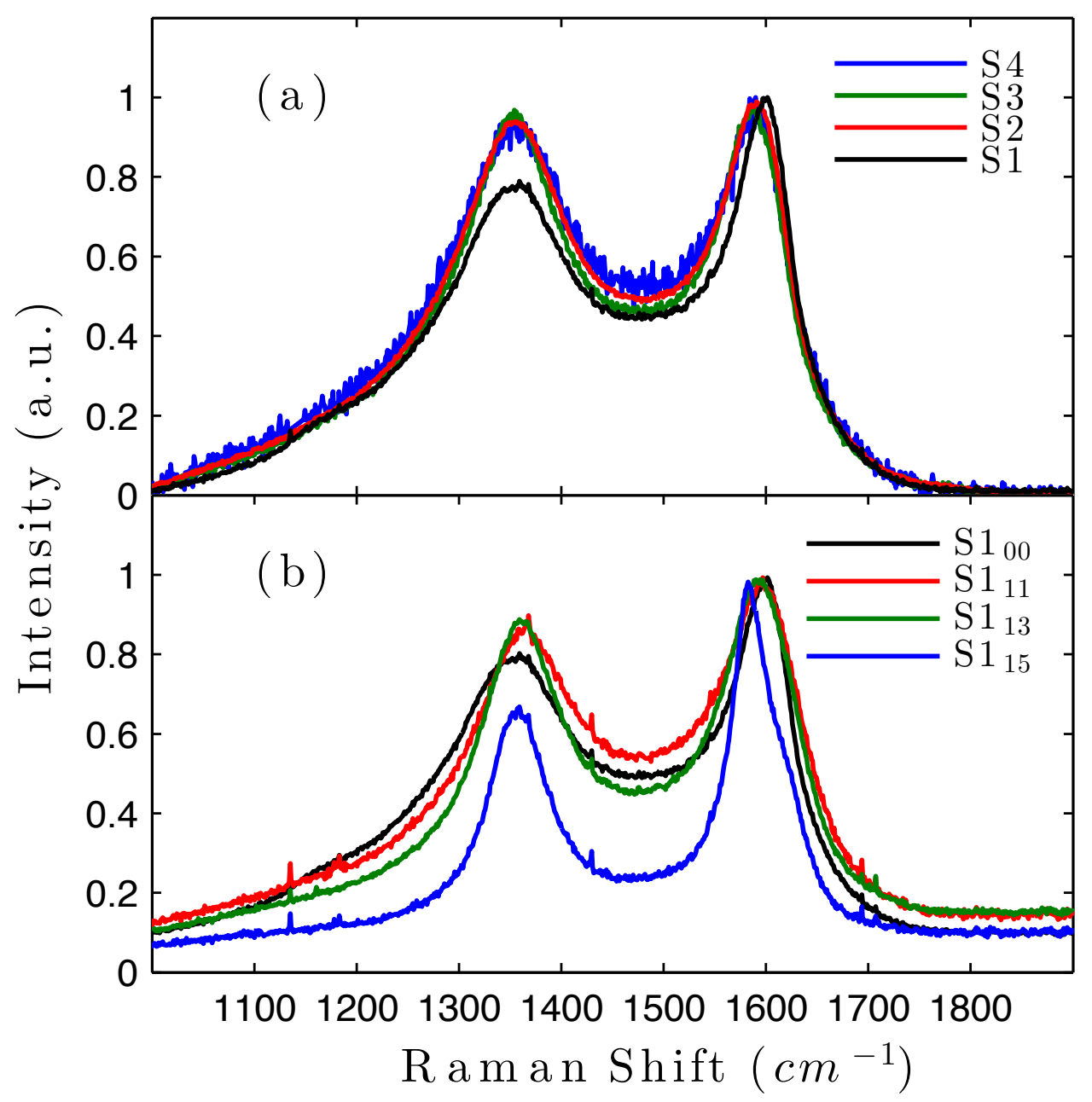

Figure 5.1: Average Raman spectra of each sample. Each spectrum was first normalized to the G peak intensity and then averaged. a) The averaged spectra of the unannealed samples. b) Averaged spectra of the annealed S1 series. 
peak and an asymmetric Lorentzian for the G peak. The cluster diameter was then calculated using the Tuinstra and Koenig equation, $[180]$

$$
\frac{I(D)}{I(G)}=\frac{C(\lambda)}{L_{a}}
$$

where $I$ is the $\mathrm{D}$ or $\mathrm{G}$ peak intensity and $C(\lambda)$ is the wavelength dependent proportionality constant. This model is applicable to nanocrystalline-graphite carbon, which makes the assumption that the sample is primarily composed of $\mathrm{sp}^{2}$ type bonds and that it is structurally closer to graphite than amorphous carbon. We make this assumption based on TEM images taken of the unannealed samples in Ref. [179] which show nanocrystallites of approximately $40 \AA . C(488 \mathrm{~nm})=35.04$ $\AA$ was calculated using the formula determined in Ref.[182]. The results are tabulated in Table 5.1. The uncertainties presented are the standard deviations of the fit parameters. However, these uncertainties should be considered minima since the complex carbon spectra makes it very difficult to fit with only two functions.

Figure 5.1 shows the average of the 10 spectra taken for each sample. Each spectra of a sample was normalized to the $G$ peak and then averaged with the other spectra of that sample. The results show how similar each of the unannealed samples are. Only the S1 is slightly different, with a slightly smaller D peak and a higher frequency G peak. Fitting the peaks results in correlation lengths in the low 40 Ås for S2 - S4 and a slightly higher length of $49 \AA$ for S1. While the $I(\mathrm{D}) / I(\mathrm{G})$ ratio suggests that $\mathrm{S} 1$ is slightly more ordered than the other samples, other aspects suggest the reverse. For instance, the S1 D peak is broader and the G peak is shifted to higher frequency. In general peak broadening suggests increased disorder, and as previously mentioned the G peak shifts to higher frequencies with disorder in Stage 1.

The annealed samples on the other hand have more variation, see Figure 5.1(b) and Figure 5.2. After annealing $\mathrm{S} 1$ at $1100{ }^{\circ} \mathrm{C}$ we see the D peak shoulder at $1150 \mathrm{~cm}^{-1}$ disappears, the D peak significantly shifts to higher frequency, a slight broadening 
TABle 5.1: Fit Parameters of each CNF Sample using a Voigt and Asymmetric Lorentzian for the D and G Peaks, Respectively

\begin{tabular}{lccccc}
\hline Sample & HTT $\left({ }^{\circ} \mathrm{C}\right)$ & D peak $\left(\mathrm{cm}^{-1}\right)$ & G peak $\left(\mathrm{cm}^{-1}\right)$ & $I(D) / I(G)$ & $L_{a}(\AA)$ \\
\hline $\mathrm{S} 1_{00}$ & - & $1350 \pm 0.7$ & $1596 \pm 0.5$ & $0.72 \pm 0.02$ & $48.9 \pm 1.0$ \\
\hline $\mathrm{S} 1_{11}$ & 1100 & $1364 \pm 0.5$ & $1595 \pm 0.4$ & $0.73 \pm 0.004$ & $48.2 \pm 0.3$ \\
\hline $\mathrm{S} 1_{13}$ & 1300 & $1361 \pm 0.9$ & $1594 \pm 0.8$ & $0.78 \pm 0.02$ & $44.9 \pm 1.2$ \\
\hline $\mathrm{S} 1_{15}$ & 1500 & $1358 \pm 0.9$ & $1589 \pm 4.7$ & $0.64 \pm 0.24$ & $63.8 \pm 32.4$ \\
\hline $\mathrm{S} 2$ & - & $1351 \pm 0.9$ & $1589 \pm 1.9$ & $0.84 \pm 0.02$ & $41.6 \pm 0.9$ \\
\hline $\mathrm{S} 3$ & - & $1350 \pm 0.9$ & $1587 \pm 0.6$ & $0.85 \pm 0.03$ & $40.9 \pm 1.2$ \\
\hline $\mathrm{S} 4$ & - & $1351 \pm 0.9$ & $1587 \pm 2.0$ & $0.85 \pm 0.05$ & $40.8 \pm 2.1$ \\
\hline $\mathrm{S} 5$ & - & $1355 \pm 0.9$ & $1594 \pm 1.1$ & $0.77 \pm 0.02$ & $45.6 \pm 1.3$ \\
\hline
\end{tabular}

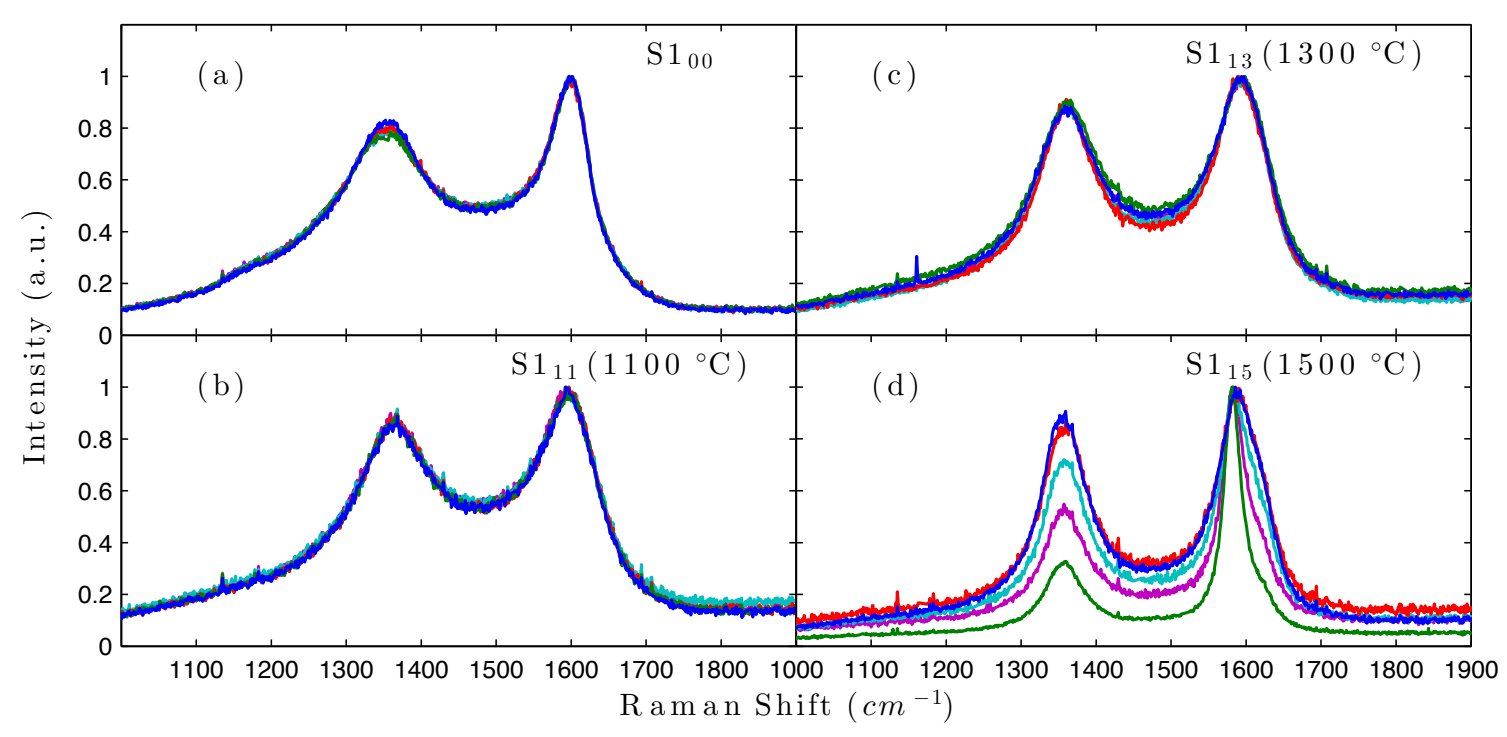

FiguRE 5.2: Five representative spectra for the samples annealed at $\mathrm{HTT} 1100^{\circ} \mathrm{C}, 1300^{\circ} \mathrm{C}$, and $1500^{\circ} \mathrm{C}$ as well as the relevant non-anneald sample showing how annealing changes the uniformity of the microstructure. a) Spectra of the unannealed sample $S 1_{00}$. b) $S 1_{11}$. The G peak broadens slightly and shoulder at $1150 \mathrm{~cm}^{-1}$ disappears. c) $\mathrm{S} 1_{13}$. Some non-uniformity of the sample begins to be present. d) $S 1_{15}$. Sample exhibits strong nonuniformity and shows signs of localized graphitization as evidenced by the strong and sharp G peak. 
and softening of the $\mathrm{G}$ peak is observed and $L_{a}$ decreases. It is difficult to address the change in ordering due to the conflicting broadening and shift of the $\mathrm{G}$ peak. The $1300^{\circ} \mathrm{C}$ annealed sample, $\mathrm{S}_{13}$, shows further $\mathrm{G}$ peak softening along with the $\mathrm{D}$ peak, and the $\mathrm{D}$ and $\mathrm{G}$ peaks narrow. This narrowing causes the reduction of $L_{a}$, because the asymmetric tail of the $\mathrm{G}$ peak is reduced causing a perceived increase in the $\mathrm{I}(\mathrm{D}) / \mathrm{I}(\mathrm{G})$ ratio and thus decrease in $L_{a}$. However, narrowing peaks are an indicator of improved crystallinity implying that $\mathrm{S}_{13}$ is less disordered than $\mathrm{S} 1_{11}$. Notice that we also begin to see a bit more variation in the spectra indicating that the sample is becoming less uniform. The largest changes are observed in the $1500^{\circ} \mathrm{C}$, most notably in the extreme variation between spectra. This indicates a further increase in the sample inhomogeneity and implies that annealing preferentially orders different parts of the sample, e.g., fibers and intersections of fibers. The width of the peaks also significantly decreases, indicating an improved crystallinity. A shoulder begins to emerge on the $\mathrm{G}$ peak at about $1610 \mathrm{~cm}^{-1}$, which is characteristic of slightly disordered graphite and multiwall carbon nano-tubes, indicating a high degree of order. The G peak also shifts to lower frequencies, again indicating an improvement in order. To further support this assessment, we see that the cluster diameter, $L_{a}$, increased by $\sim 50 \%$. Note the large uncertainty of $L_{a}$, this is because the calculated cluster diameter ranges from 40 to $118 \AA$ due to the inhomogeneity of the sample.

In conclusion, we see very little difference in the microstructure of the as grown samples, but the annealed samples show progressive improvement in structural quality with annealing temperature. While only the highest temperature annealed sample showed quantitative crystalline improvement due to the difficulty of fitting, it can be reasonably inferred from qualitative arguments that the microstructure improves with annealing temperature.

SAXS data was acquired through the user program at Oak Ridge National Laboratory using Anton Paar's SAXSess $\mathrm{mc}^{2}$ tool operating at $40 \mathrm{kV}$ and $30 \mathrm{~mA}$ with the incident beam line collimated. Data was collected on imaging plates and 


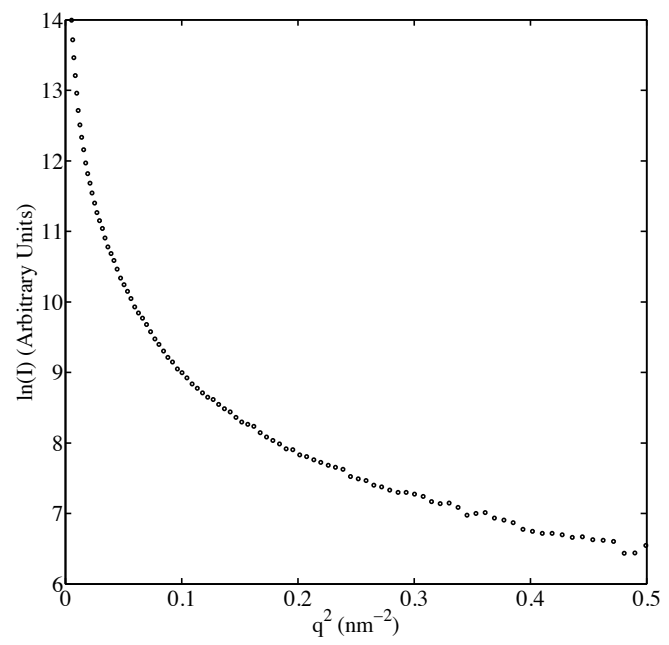

FIGURE 5.3: Guinier plot of SAXS intensity decay. The lack of linear region over this $q$ regime indicates an absence of monodisperse voids observed in other carbon fiber samples.

desmeared prior to analysis. X-ray scattering experiments measure the angle, $\theta$, and intensity, $I$, with which incident radiation scatters from a sample. The exit angle is considered in terms of the wave form vector: $q=4 \pi / \lambda \sin (\theta)$ with the $\mathrm{x}$-ray wavelength $\lambda=1.5418 \AA$. This diffuse scattering is caused by spatial fluctuations in electron density, allowing it to be used to characterize the nanostructure of multi-phase systems. The scattered x-rays' exit angle corresponds to features of regularly occurring particles. Larger particle features influence the data at smaller angles. Ideally, SAXS experiments complement microscopy experiments by identifying a statistically significant average of the feature of interest.

In the case of carbon fibers, previous works have demonstrated an ability to characterize the dimensions of the voids contained within the individual fibers.[174, 183] These studies looked at systems with long crystalline regions, creating the opportunity for strain to pull the planes of carbon apart. In the same manner, a Guinier plot of the data is generated in the low angle regime in Figure 5.3. From the slope and intercept of such a plot, it is possible to determine the radius of gyration and cross-sectional area of an included particle (or void) of appropriate size in the system due to variations in electron density between the 
included particle and host material. However, the lack of a linear region in our data indicates voids similar to those observed by other authors do not exist. Transmission electron microscopy of similar turbostratic samples help confirm this result.[179] Data taken on each fabricated sample displayed nearly identical trends to that shown in Figure 5.3. It is concluded that voids with diameters on the order of a few nanometers do not exist in these samples. This result simplifies thermal modeling considerations. Nanoscale voids could vary with heat treatment or pressure applied to the system and therefore vary the scattering rate of thermal energy carries.

Scanning electron microscope images of the samples are shown in Figure 5.4 which displays the highly entangled nature of the fiber composites, relatively low fill fraction, and regularity of fiber to fiber contacts. From these images a fiber diameter on the order of $100 \mathrm{~nm}$ is inferred. This value is used to estimate pore size[36] and therefore gas thermal conductivity, $k_{g}$. Additionally, rod diameter influences the Hertzian contact radius which varies fiber to fiber contact conductance as pressure is applied. These images give confidence to the assumption of random fiber orientation used to model the network.
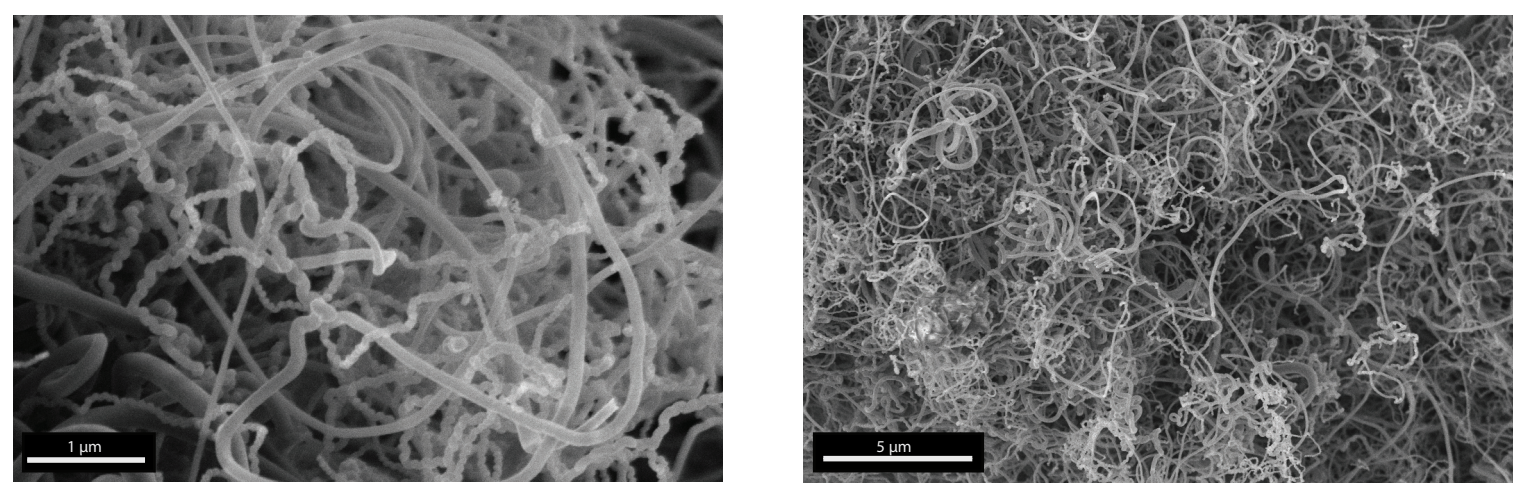

FiguRE 5.4: Scanning electron microscope image of a carbon fiber sample. 


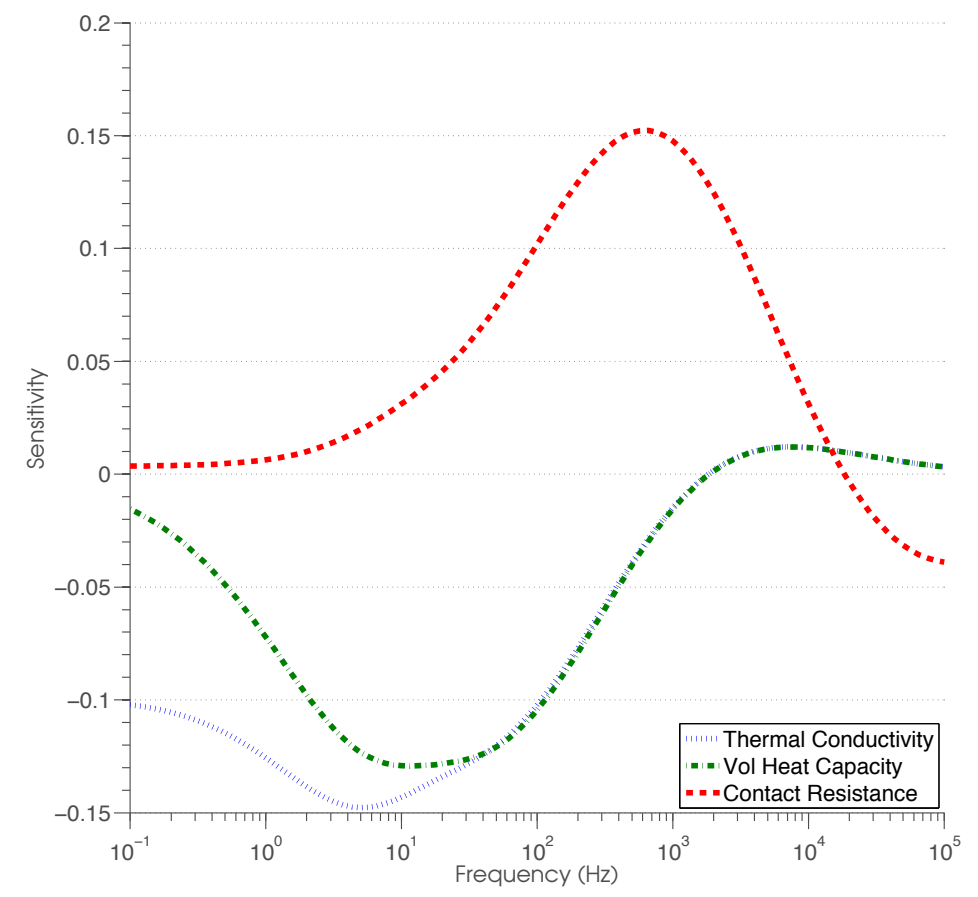

FIGURE 5.5: Sensitivity of the $3 \omega$ experiment to composite samples' effective thermal conductivity and volumetric heat capacity as well as the contact resistance between the mount and sample. This plot was generated with $k_{e}=0.024 \mathrm{~W} / \mathrm{m} \mathrm{K}, C_{e}=3 \times 10^{5} \mathrm{~J} /\left(\mathrm{m}^{3} \mathrm{~K}\right)$, and $R_{p}=6 \times 10^{-5} \mathrm{~K} \mathrm{~m}^{2} / \mathrm{W}$, similar to the fourth data point in Figure 5.6. The change in direction of the contact resistance sensitivity above $10,000 \mathrm{~Hz}$ only exists due to the presence of the $\mathrm{SiO}_{x}$ layer closer to the heater. At greater applied pressures, the signal's sensitivity to the carbon fibers' thermal conductivity and heat capacity is increased. At low applied pressures the experiment can lose sensitivity to these properties.[4] . 


\subsection{Thermal characterization and modeling}

The bidirectional $3 \omega$ technique described in Chapter 3 was utilized to characterize the thermal conductivity and volumetric heat capacity of the carbon fiber samples. This bidirectional variation of the $3 \omega$ technique was necessary due to the difficulty of depositing requisite linear thin metal wire directly onto the samples, and due to the fact that it would be impossible to maintain constant metal line dimensions as pressure is applied and the carbon fiber sample is compressed. As discussed in Chapter 3, the metal line which heats the sample and detects the resulting temperature rise rests on an $\mathrm{Su}-8$ polymer with a copper layer serving as the base. $\mathrm{An}_{\mathrm{SiO}}$ passivation layer $240 \mathrm{~nm}$ thick was deposited above the heating wire. This layer electrically isolates the carbon fiber sample from the metal line. The properties of the polymer and passivation layer must be independently verified. The thermal conductivity of the $\mathrm{SiO}_{x}$ layer is determined be $0.91 \mathrm{~W} / \mathrm{m} \mathrm{K}$. Previous studies have measured $\mathrm{SiO}_{2}$ thin films slightly below $1 \mathrm{~W} / \mathrm{m} \mathrm{K}$ near $200 \mathrm{~nm}$ thickness.[69]. The heating line was used to test the thermal conductivity of the polymer layer under vacuum without the carbon fiber sample present. This is similar to traditional $3 \omega$ experiments. [74] This experiment yielded a thermal conductivity of $0.14 \mathrm{~W} / \mathrm{m} \mathrm{K}$ which is similar to the advertised thermal conductivity from the manufacturer, MicroChem, of $0.2 \mathrm{~W} / \mathrm{m} \mathrm{K}$ as well as that measured by others.[184] With these inputs known, the thermal properties of the carbon fiber networks can be characterized with three fitting parameters: the samples' effective thermal conductivity and volumetric heat capacity, as well as the contact resistance between the passivation layer and the sample $\left(k_{e}, C_{e}\right.$, and $R_{p}$ respectively). Each sample is pressed onto the passivation layer directly above the heating line with a plate on the backside of the sample. Pressure is applied by tightening screws connecting this plate to the sample mount. Properties of the screws, and the torque required to tighten each screw are used to approximate the pressure applied to the sample. Heat travels from the metal wire into both the polymer layer as well as the sample. Frequency sweeps from 1 to $10,000 \mathrm{~Hz}$ allow the simultaneous 
fitting of each parameter. Data were analyzed accounting for bidirectional heat flow from the heating wire.[4, 74, 87] Due to the length scale of the samples tested and the small temperature rise necessary for $3 \omega$ experiments, the effects of black body radiation can be approximated as less than $2 \%$.[66]

Figure 5.5 demonstrates the unique sensitivity to each parameter for the frequency range tested, indicating the three parameters can be fit simultaneously. As discussed in Chapter 3, the sensitivity parameter is defined as

$$
S_{p}=\frac{\left(V_{3 \omega}(\Delta)-V_{3 \omega}(0)\right) / V_{3 \omega}(0)}{(\Delta p-p) / p}=\frac{V_{3 \omega}(\Delta)-V_{3 \omega}(0)}{V_{3 \omega} \Delta}
$$

in which parameter of interest $p$ is perturbed by amount $\Delta$ for which $10 \%$ is selected. As can be seen, each fitting parameter exhibits a unique sensitivity trend. Both $k_{e}$ and $C_{e}$ become uniquely identifiable below $100 \mathrm{~Hz}$, and particularly below $10 \mathrm{~Hz}$ at which point the contact resistance sensitivity effects become minimal. At lower frequencies the measured third harmonic waves are sensitive to properties deeper into the sample, making the relative contribution of the contact resistance negligible. However at higher frequencies, thermophysical properties closer to the heating wire dominate the signal, causing greater sensitivity to the contact resistance and properties of the passivation layer. The change in direction of the contact resistance sensitivity above $10,000 \mathrm{~Hz}$ only exists due to the presence of the $\mathrm{SiO}_{x}$ layer closer to the heater. At greater applied pressures, the signal's sensitivity to the carbon fibers' thermal conductivity and heat capacity is increased. At low applied pressures the experiment can lose sensitivity to these properties.[4] The experiment was repeated on each sample with the loading pressure systematically varied. As the axial loading pressure was increased, the density of the sample increased, altering the properties of the sample and the contact resistance. This is easily identified by observing the third harmonic voltage, $V_{3 \omega}$, decrease at a given driving frequency as the applied pressure increases. This indicates the temperature 
rise at the heating line, $T_{2 \omega}$, has decreased due to an increased rate of heat diffusion through the system.

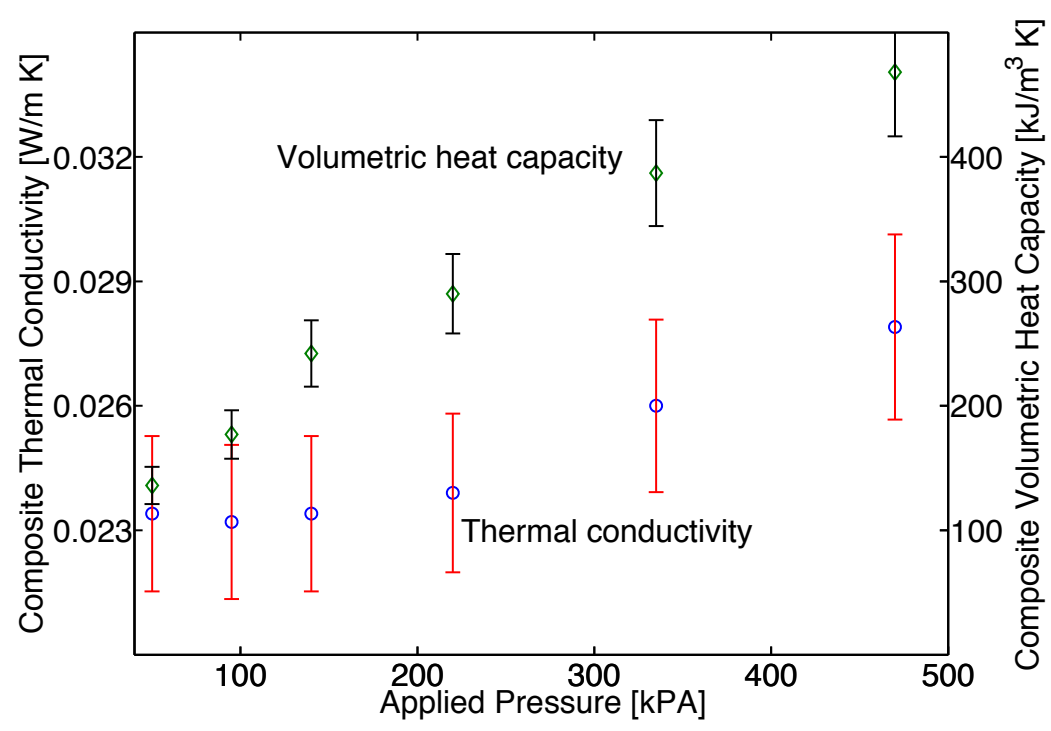

Figure 5.6: Best fit results of data taken on $\mathrm{S}_{100}$ with measured density of $90 \mathrm{~kg} / \mathrm{m}^{3}$. Measurements were taken at six unique compressive pressures, with the sample thermal conductivity, sample volumetric heat capacity, and the sample to mount contact resistance simultaneously fit. This data is representative of data taken on each sample. The fitting error propagated from sources of uncertainty in the system is estimated at $9 \%$ for the sample's thermal conductivity, $11 \%$ for the volumetric heat capacity and $6 \%$ for the contact resistance between the sample and the passivation layer.

Figure 5.6 displays the fitted results for data taken at atmospheric pressure on the portion of S1 which did not undergo heat treatment. The volumetric heat capacity, $C_{e}$, varies linearly with pressure. This trend can be attributed to a change in density of the composite when the macroporous voids shrink as the sample is compressed. It is assumed that the heat capacity per unit mass of the nonwoven carbon nanofibers, $c_{m}$, is approximately $710 \mathrm{~J} / \mathrm{kg} \mathrm{K}$. With this information the fill fraction of the sample during each measurement can be approximated:

$$
\phi=C_{e} /\left(c_{m} \rho_{0}\right)
$$

A loading pressure ranging from 50 to $470 \mathrm{kPa}$ caused the density of the $90 \mathrm{~kg} / \mathrm{m}^{3}$ 
sample (implying a $\phi_{0}=5 \%$ fill fraction) to vary from $15 \%$ to over $40 \%$ fill fraction. Over this pressure range, the best fit contact resistance varied from $10^{-4}$ to $5 \times 10^{-5}$ $\mathrm{m}^{2} \mathrm{~K} / \mathrm{W}$. Also indicated in Figure 5.6 is the gradual increasing trend of the effective sample thermal conductivity as greater pressure is applied. This result is representative for all samples which did not undergo heat treatment. Using Equation 5.3, and the measured volumetric heat capacity, the carbon fiber fill fraction is able to be inferred at each pressure, $\mathrm{P}$ (that is $\phi(P)$, which is some value greater than $\left.\phi_{0}\right)$. This changing fill fraction can be used to approximate structural parameters which change as pressure is applied. Knowledge of these parameters (such are pore size, fiber orientation, fiber contact density, etc.) is subsequently used to understand the measured thermal conductivity values on each sample in each scenario.

To understand the best fit thermal conductivity values, the properties of the carbon fibers, fiber to fiber interactions, and the air host are considered. Previous work has measured the thermal conductivity of individual carbon fibers with short coherence length.[171] Qiu et al. provide a linear fit of thermal conductivity vs coherence length which leads to an approximation of the intrinsic thermal conductivity of the individual fibers. The non heat treated samples tested in this work have an estimated intrinsic fiber conductivity near $40 \mathrm{~W} / \mathrm{m} \mathrm{K}$ based on this linear approximation and the cluster diameter determined from the Raman measurements.

For fibrous insulation type materials of similar densities and length scales, Bhattacharyya has demonstrated that the contribution of convection is negligible.[185] Conduction through the air phase is a function of temperature, the pressure of the atmosphere, and the air molecules' mean free path (MFP) between scattering events, $\lambda$. For air in an infinitely large medium the bulk mean free path $\left(\lambda_{B}\right)$ is $68 \mathrm{~nm}$ at standard temperature and pressure, which results in the thermal conductivity of air of $0.026 \mathrm{~W} / \mathrm{m} \mathrm{K.[186]}$ The MFP of air in entangled fibrous 
networks can be approximated[36] as $\lambda_{n}=\frac{\pi r}{2 \phi}$ where $r$ is the fiber radius which is approximated as $50 \mathrm{~nm}$ from the SEM images. As the Knudsen number increases $\left(K n=\lambda_{B} / \lambda_{N}\right)$, the thermal conductivity of the gas decreases. Using modeling by Raed and Gross, [39] the thermal conductivity of the gas phase $\left(k_{g}\right)$ of our samples at the smallest applied axial load pressure is $0.009 \mathrm{~W} / \mathrm{m} \mathrm{K}$. This value decreases with added pressure down to $0.005 \mathrm{~W} / \mathrm{m} \mathrm{K}$ at the highest applied pressures tested due to decreasing pore size and therefore increasing scattering rate of the air molecules. In the case of $\mathrm{S} 5$ tested at vacuum pressure of $0.1 \mathrm{~Pa}$, the thermal conductivity of the gas phase at these reduced dimensions is negligible.[186]

Using this information, we can compare our data to basic thermal conductivity models. Calculating the composite's effective thermal conductivity, $k_{e}$, using a simple rule of mixing as the weighted average of the two phases, $k_{e}=\phi k_{f}+(1-\phi) k_{g}$, over predicts our results by two orders of magnitude. To gain insight into the origins of this reduction of thermal conductivity, we consider the microstructure of the sample. Fricke's method for predicting electrical conductivity of disperse suspensions[187] can be used to predict the thermal conductivity of rods if the sample can be assumed as a homogenous medium with fiber interaction averaged over the unit cell volume.[185] By defining the ratio of thermal conductivity, $C_{r}=k_{g} / k_{f}$, and the volume ratio $V_{r}=$ $\rho /\left(\rho_{0}-\rho\right)$, Bhattacharyya used Fricke's method to predict the thermal conductivity of a composite with fibers perpendicular to heat flow as [185]

$$
k^{B}=k_{f}\left(1-\frac{1-C_{r}}{1+\left(2 C_{r} V_{r}\right) /\left(1+C_{r}\right)}\right)
$$

This model predicts the correct order of magnitude compared to the measurements in this work but fails to capture the trend as pressure is applied. Stark and Fricke build upon Bhattacharyya's model by first modifying the equation for the representative 
unit cell to be a function of average fiber orientation, $Z$ :[188]

$$
k^{B Z}=k_{f}\left(1+\frac{C_{r}+1}{1+V_{r}\left(1+Z\left(C_{r}-1\right) /\left(C_{r}+1\right)\right)}\right)
$$

Stark and Fricke then advance the model by taking into account the fiber to fiber interactions as part of a resistor network. The effective thermal resistance from fiber to fiber contacts in a unit cell is[188]

$$
R_{c t}=\frac{j \pi a_{c t}}{4 k_{f} A r}
$$

where $r$ is the fiber radius, $A$ is a connection parameter equal to $0.611, j$ is a parameter defining the unit cell area, and $a_{c t}$ is the radius of the contact between the fibers. The resistance due to an individual fiber to fiber contact is estimated to be on the order of $10^{5} \mathrm{~K} / \mathrm{W}$. For reference, others studying carbon nanotube contact resistances without an external applied compressive force and with rod diameter an order of magnitude smaller find contact resistances on the order of $10^{7} \mathrm{~K} / \mathrm{W} .[189,190]$ By incorporating $R_{c t}$ and $k^{B Z}$ into a resistor network, and leaving $Z$ as a fitting parameter, Stark and Fricke had success matching their thermal model to a large variety of thermal conductivity data for insulation type materials with $Z$ varying from 0.66 to 0.97 .

To accurately apply the Stark and Fricke Model (SFM) to describe thermal transport in each sample, the parameters that vary as pressure is applied must be taken into account: $Z, \phi, k_{g}$, and $R_{c t}$. As previously stated, the fiber fill fraction, $\phi(P)$, is determined from the best fit volumetric heat capacity and gas conductivity, $k_{g}$, is approximated from previously developed models.[39] For any fibrous composite, the average fiber orientation is difficult to independently measure. When all rods are perpendicular or parallel to the flow of heat, $\mathrm{Z}=1$ or $\mathrm{Z}=0$, respectively, while $\mathrm{Z}=2 / 3$ indicates completely random orientation in three dimensional space. To avoid attempting to overfit the data, the average fiber is assumed to be completely randomly oriented, and $Z=2 / 3$ is used in all scenarios prior to pressure 
being applied to the sample. It is then assumed that as pressure is applied to the samples, the increased density incorporates new perpendicular rods while removing rods parallel to heat flow: i.e., bending rods over. Therefore, the increase in $Z$ at each successive pressure can be calculated as a function of the changing density of the sample, as determined from the fit volumetric specific heat. Let the ratio of perpendicular to parallel rods at any pressure be $R_{Z}(n)$ and $Z(0)$ be the orientation when no pressure is applied. Then, at each increased density, the ratio can be calculated and used to determine the new average fiber orientation:

$$
\begin{gathered}
R_{Z}(n)=\frac{\phi(0) Z(0)+\phi(n)-\phi(0)}{(1-Z(0)) \phi(0)} \\
Z(n)=R_{Z}(n) /\left(R_{Z}(n)+1\right)
\end{gathered}
$$

Finally, the path of thermal conductance between fiber contacts varies as pressure is applied: the size of the representative unit cell is altered (and the cell density), the contact area between the fibers varies, and the individual fiber to fiber contact resistance also varies as function of pressure. For a bed of fibers, the Hertzian contact radius can be calculated[36]:

$$
a_{c t}=\left(\frac{3}{2} \frac{\left(1-\mu^{2}\right)}{E} P_{1} r\right)^{1 / 3}
$$

in which $\mu$ is Poisson's ratio, $E$ is Young's modulus, and $P_{1}$, the load on an individual contact, is a function of the applied pressure, $p$ :

$$
P_{1}=p\left(\frac{\pi r}{2 \phi Z}\right)^{2}
$$

Knowing the applied pressure and contact radius, Equation 5.6 can be used to determine the effective contact resistance through the fiber to fiber contacts. With $R_{c t}, Z, \phi, k_{f}$, and $k_{g}$ determined, the SFM can be used to calculate the thermal conductivity and the results can be compared directly to the measured $3 \omega$ results 
without fitting parameters.

Figure 5.7 demonstrates the isolated effects of fiber orientation, density, air conductivity, and the contact resistance term on the modeled thermal conductivity (each term that varies as pressure is applied) for a sample with an initial density of $200 \mathrm{~kg} / \mathrm{m}^{3}$ where $k_{f}=40 \mathrm{~W} / \mathrm{m} \mathrm{K}$ has been assumed. The positive effective thermal conductivity trend when pressure is applied can only be a result of the interactions between touching fibers. Other models which predict the effective thermal conductivity of two phase media that do not take into account the interactions of the included particles, such as effective medium theory, cannot replicate this trend. In other scenarios in which the sample has different properties, such as assuming an increased intrinsic fiber conductivity, or higher percentage of fibers parallel to heat flow, the response of $k_{e}$ to fiber fill fraction, $\phi$, is positive when pressure is applied.

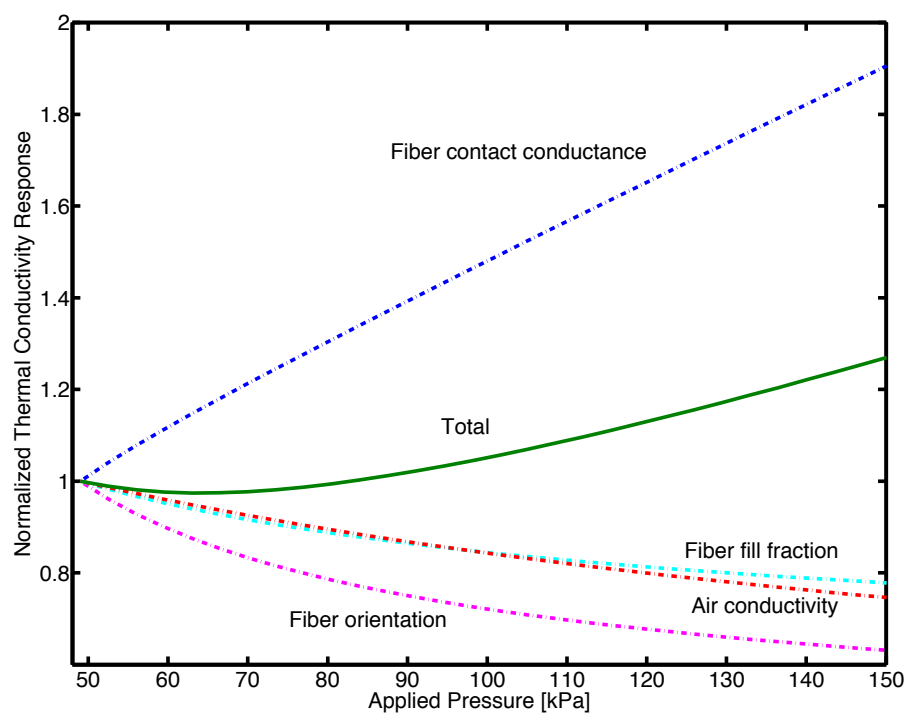

FiguRE 5.7: Normalized modeled response of $200 \mathrm{~kg} / \mathrm{m}^{3}$ dense carbon nanofiber composite (S5) as pressure is applied. The total thermal conductivity response, $k_{e}$, only increases due to the role of thermal conductance through fiber contacts. Changing fiber orientation, fill fraction, and air conductivity each act to decrease the composite's thermal conductivity as pressure is applied.

Figure 5.8 displays the best fit sample thermal conductivity, $k_{e}$, of S5 at atmospheric pressure and under vacuum plotted against the calculated carbon fiber 


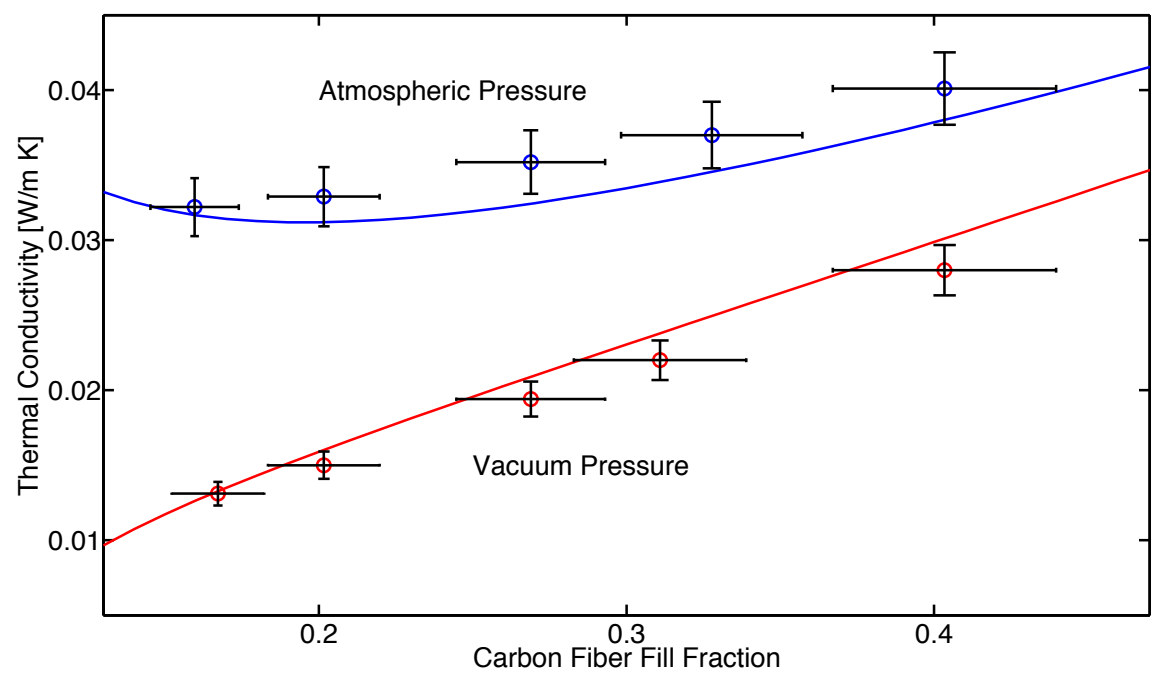

FiguRE 5.8: Measured thermal conductivity values from sample S5 at both vacuum and atmospheric pressure. Carbon fill fraction is calculated from the best fit volumetric heat capacity using Equation 5.3 .

fill fraction, $\phi$, as determined from the best fit volumetric specific heat of the sample. Results of the SFM are also plotted in Figure 5.8 with the appropriate inputs recalculated at each pressure. When no pressure is applied, $\phi=12 \%$. As highlighted by Figure 5.7 the increase in thermal conductivity as pressure is applied is a result of the increase in the conductance path between interacting fibers. The relative shift between thermal conductivity at atmospheric pressure and vacuum pressure decreases at higher fill fraction. At atmospheric pressure, shrinking pore size decreases the MFP of air molecules (increasing the Knudsen number) ultimately causing conduction through the gas to contribute a smaller portion to the sample's effective thermal conductivity. In the data series taken at vacuum pressure, $k_{g}$ is negligible at each data point. In the limit, as the MFP of the air approaches zero due to infinitely small pores, the thermal conductivity of the samples measured at atmospheric conditions would approach the same value as the measured result with the sample held under vacuum.

Measurements of samples having variable initial fill fraction further underscore 


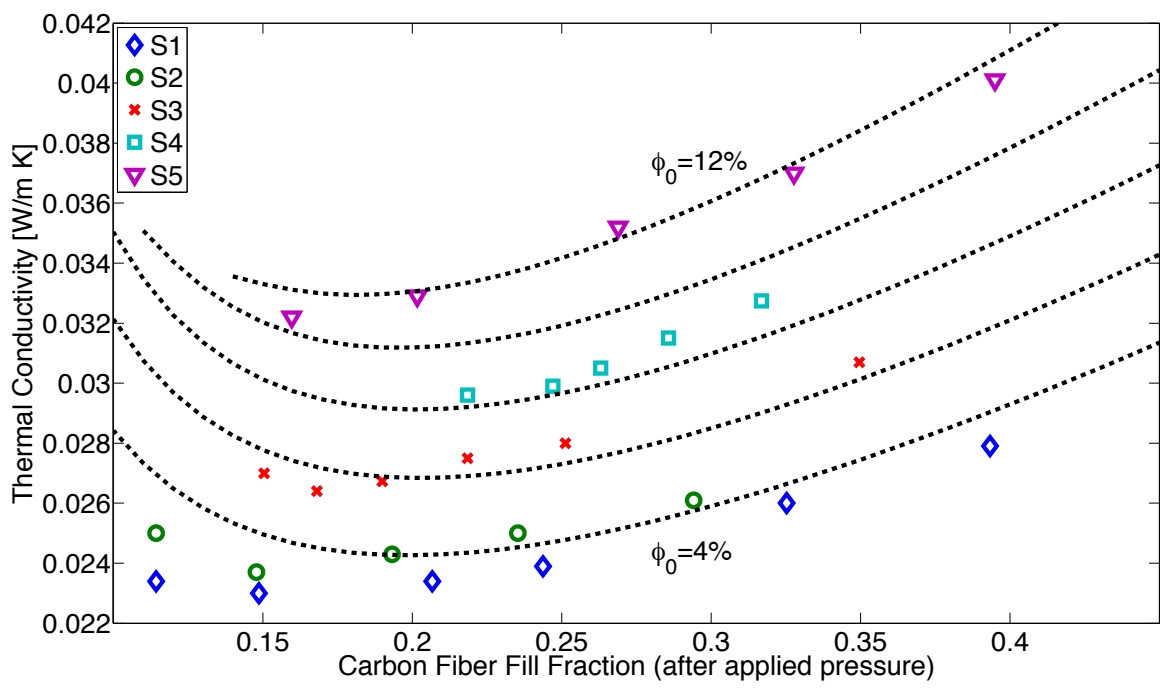

Figure 5.9: Best fit $3 \omega$ thermal conductivity results for non heat treated samples S1, S2, S3, S4, and S5 (densities of 90,110,130, 160 and $200 \mathrm{~kg} / \mathrm{m}^{3}$ respectively). All data is acquired at room temperature and atmospheric pressure. The thermal conductivity predictions from the SFM (dashed lines) are also plotted for initial fill fractions ranging from $4 \%$ to $12 \%$ in steps of $2 \%\left(\phi_{0}\right)$. As pressure is applied the fill fraction increases to a maximum value of $\phi(P)=40 \%$.

the utility of the SFM as shown in the data of Figure 5.9. Again, the SFM with the discussed parameter trends is plotted over the best fit results (dashed lines), however, the input initial fill fraction, $\phi_{0}$, value used for the model is systematically changed from $4 \%$ to $12 \%$ in increments of $2 \%$. By accounting for each changing parameter, the SFM accurately predicts both the magnitude of $k_{e}$ and the trend as pressure is applied. Interestingly, the thermal conductivity of the samples with low initial density at high applied pressure (e.g. $\mathrm{S}_{1}$ at $\phi=40 \%$ has $k_{e}=0.027 \pm 0.0024 \mathrm{~W} / \mathrm{m} \mathrm{K}$ ) remains significantly less than samples with higher initial fill fraction when minimal pressure is applied (e.g. $\mathrm{S}_{5}$ at $\phi=16 \%$ has $k_{e}=0.032 \pm 0.0028 \mathrm{~W} / \mathrm{m} \mathrm{K}$ ). It is apparent that for material systems of similar intrinsic properties, the initial fill fraction of the system is more important than the resulting fill fraction if a compressive force is applied to the system while it is operating. Even though at increased applied pressures there is increasing conductance between touching rods, rods changing from near parallel to near perpendicular to the heat flux can nearly negate this contribution. 


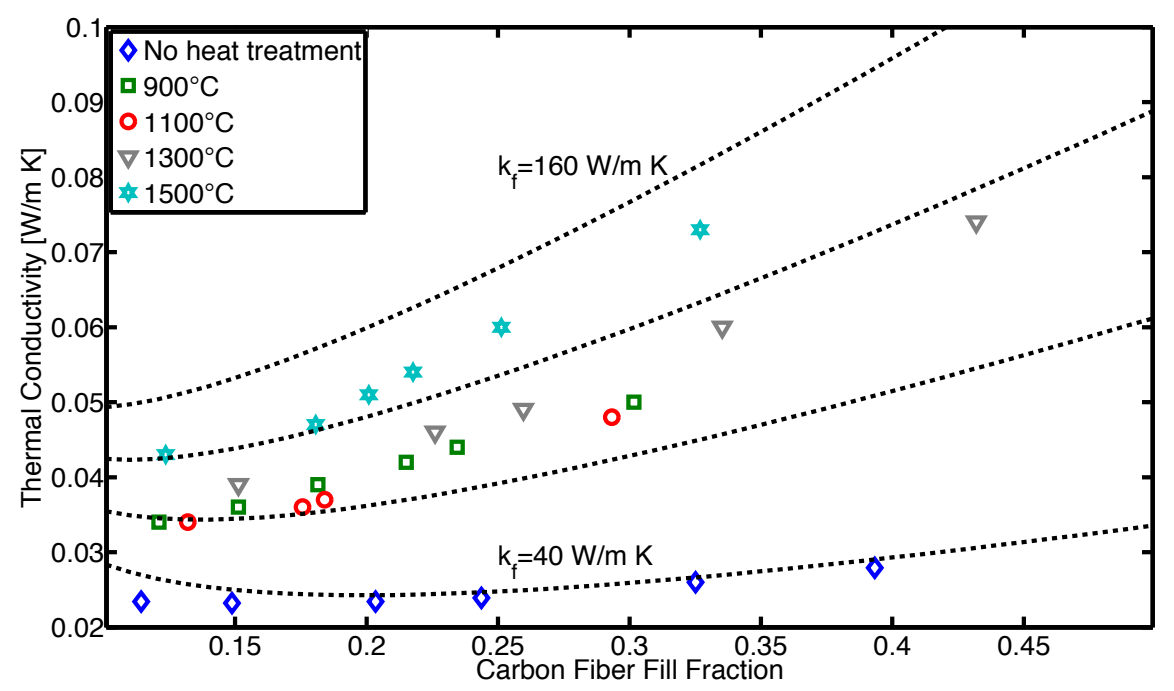

Figure 5.10: Best fit data from the heat treated sample series (S1). The measured thermal conductivity increases significantly from the as grown sample compared to the heat treated samples, with the sample which underwent a $1500^{\circ} \mathrm{C} \mathrm{HTT}$ showing an additional increase compared to the more moderately heated samples. The change in effective thermal conductivity with respect to carbon fiber fill fraction has increased with heat treatment. Over the data the SFM is plotted with carbon fiber thermal conductivities of $40,80,120$, and $160 \mathrm{~W} / \mathrm{m} \mathrm{K}$ respectively.

Lastly, the role of intrinsic fiber thermal conductivity is examined using the dependence of thermal conductivity on heat treatment as shown in Figure 5.10. Similar to Figure 5.9 the SFM is plotted over this data. Here the input fiber thermal conductivity, $k_{f}$, is systematically varied from 40 to $160 \mathrm{~W} / \mathrm{m} \mathrm{K}$ to reflect the anticipated change resulting from the heat treatment process on the samples. As expected, heat treating results in a measurable increase in sample thermal conductivity, $k_{e}$, which is likely caused by increasing $k_{f}$. This result is most apparent at the $\mathrm{HTT}$ of $1500^{\circ} \mathrm{C}$ in which we infer $k_{f}$ has increased to around 120 $\mathrm{W} / \mathrm{m} \mathrm{K}$. This agrees with the Raman measurements indicating crystalline coherence length has increased compared to the as grown samples. The three samples heat treated at maximum temperatures of $900^{\circ} \mathrm{C}, 1100^{\circ} \mathrm{C}$, and $1300^{\circ} \mathrm{C}$ showed a more moderate increase compared the non-heat treated sample, with an apparent fiber thermal conductivity around 80 to $100 \mathrm{~W} / \mathrm{m} \mathrm{K}$. The increased intrinsic fiber conductivity causes a more rapid rise in the effective sample thermal conductivity as 
pressure is applied. Over the relatively moderate pressure range tested, the $1500^{\circ} \mathrm{C}$ HTT sample exhibited a 50\% thermal conductivity increase while the non-heat treated samples exhibited increases closer to 20\%. Composites with high intrinsic conductivity fibers increase in conductivity more rapidly as pressure is applied.

\subsection{Conclusions}

Fiber networks are increasingly being utilized in microsytsems requiring thermal management. Predicting thermal transport in composite systems requires an understanding of each phase as well as their interactions. Predictive models that do not take into account interactions between the individual fibers do a poor job of anticipating thermal transport in networks of fibers. Through thermal conductivity measurements under varying compressing pressures on composite carbon fiber networks, it is shown that the thermal pathway between touching fibers must be accounted for to predict increased thermal transport as greater pressure is applied. Beyond this, methods for accounting for other contributions to thermal transport in composites are highlighted including the role of the fluid within the composites pores and the accounting for fibers bending as pressure is applied. The strong influence of the initial fiber fill fraction is shown to be often more important to minimizing thermal transport than the fill fraction after compression. Finally, the intrinsic fiber conductivity displays an increasingly strong influence on the composite's thermal conductivity at greater applied pressures for systems of similar initial fill fractions. 


\section{Chapter 6}

\section{Vertically Aligned Carbon \\ Nanotube Arrays for Heat \\ Removal}

Content and images from this chapter have been published as "Thermal conductivity of vertically aligned carbon nanotube arrays: Growth conditions and tube inhomogeneity" by M. L. Bauer, Q. N. Pham, C. B. Saltonstall, and P. M. Norris.[191]

\subsection{Background}

Studies presented up to this point have focused on material systems with uniquely low thermal conductivity ranges combined with other desired structural and mechanical properties. Expanding the upper limit of thermal conductivity is equally if not more challenging, particularly while achieving other desired system properties. Allotropes of carbon present much promise in this area. Strong carbon $\mathrm{sp}^{2}$ and $\mathrm{sp}^{3}$ as well as hybridized bonds transport thermal energy at a uniquely fast rate in part due to a low phonon scattering rate. Diamond, in which carbon atoms 
arrange in a face-centered cubic crystal pattern via $\mathrm{sp}^{3}$ bonds, exhibits a thermal conductivity over $1,000 \mathrm{~W} / \mathrm{m} \mathrm{K}$ at room temperature.[163] However, the extreme hardness of diamond is not favorable in many thermal stability applications. Graphene, (a single monolayer of graphite) composed of $\mathrm{sp}^{2}$ bonds has measured

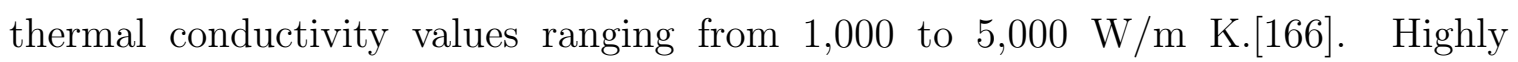
oriented pyrolitic graphite has room temperature in-plane conductivity just under $2,000 \mathrm{~W} / \mathrm{m} \mathrm{K}$.

Carbon nanotubes (CNTs) are structurally a sheet of graphene which have been wrapped into a cylinder. The angle of this wrap has a strong influence on the properties of the CNT. Multiple concentric CNTs can be wrapped together to make a multi wall carbon nanotube (MWNT) which can be over a hundred layers thick. CNTs are sought in thermal management applications in part due to their mechanical properties including a tensile strength between 10 and $60 \mathrm{GPa}$ and Young's modulus ranging from 200-1,000 GPa.[192] Individual CNTs have been shown to exhibit very large intrinsic thermal conductivity due to the often quasi-ballistic phonon transport within the tubes caused by the strong $\mathrm{sp}^{2}$ bonds and unique microstructure.[193]

As mentioned in Chapter 1, materials with such elastic properties combined with large thermal conductivity values are ideal as thermal interface materials (TIMs) to assist with removing heat from integrated circuits.

As mentioned in Chapter 1, materials with such elastic properties combined with large thermal conductivity values are ideal as thermal interface materials (TIMs) to assist with removing heat from integrated circuits. Vertically aligned CNT arrays (VACNTs) are strong candidates for use as TIMs in microdevices to aid in removing heat from the electronic components while avoiding delimitation with the heat sink. However, the promising thermal performance of individual nanotubes has not been fully realized in usable carbon nanotube composite materials.[194-196] Previous studies have reported individual carbon nanotube (CNT) thermal conductivities on 
the order of $3,000 \mathrm{~W} / \mathrm{m} \mathrm{K}$ at room temperature.[197-199] The values vary with tube length[200, 201], defect density[202, 203], and concentric tube count[204]. However, when scaling from individual CNTs to aligned bundles that may be usable in devices, authors have identified a significant decrease in sample thermal conductivity. [205] This decrease is greater than expected if one only considered the thermal conductivity of individual CNTs and the volume fraction of the system which is occupied.[206] Designing next generation TIMs with VACNTs requires an improved thermal conductivity beyond that of reported values. The origin of the reduction of thermal conductivity in VACNTs is an ongoing area of investigation.

In this work, the thermal conductivity of VACNTs grown on silicon dioxide substrates via chemical vapor deposition is measured using a $3 \omega$ technique. For each sample the VACNT layer and substrate are pressed to a heating line at varying pressures to extract the sample's thermophysical properties. The nanotubes' structure is observed via transmission electron miscroscopy and Raman spectroscopy. The presence of hydrogen and water vapor in the fabrication process is tuned to observe the effect on measured thermal properties.

\subsection{VACNT growth}

The VACNTs studied here are synthesized via chemical vapor deposition[207] in a tube furnace. A $12 \mathrm{~mm}$ by $12 \mathrm{~mm}$ amorphous $\mathrm{SiO}_{2}$ substrate is placed within a horizontal quartz tube of $20 \mathrm{~mm}$ inner diameter and over a meter long. As depicted in Figure 6.1 the growth chamber is connected to several gases via mass flow controllers (MFC). We seek to tune the thermal properties of the samples by varying the introduction and flow rate of water vapor as well as hydrogen into the system during synthesis to generate eight unique samples. In all scenarios ethylene serves as the carbon source and is introduced into the system at a flow rate of 100 sccm. For the control sample, labeled Sample A, the chamber is prepared by 


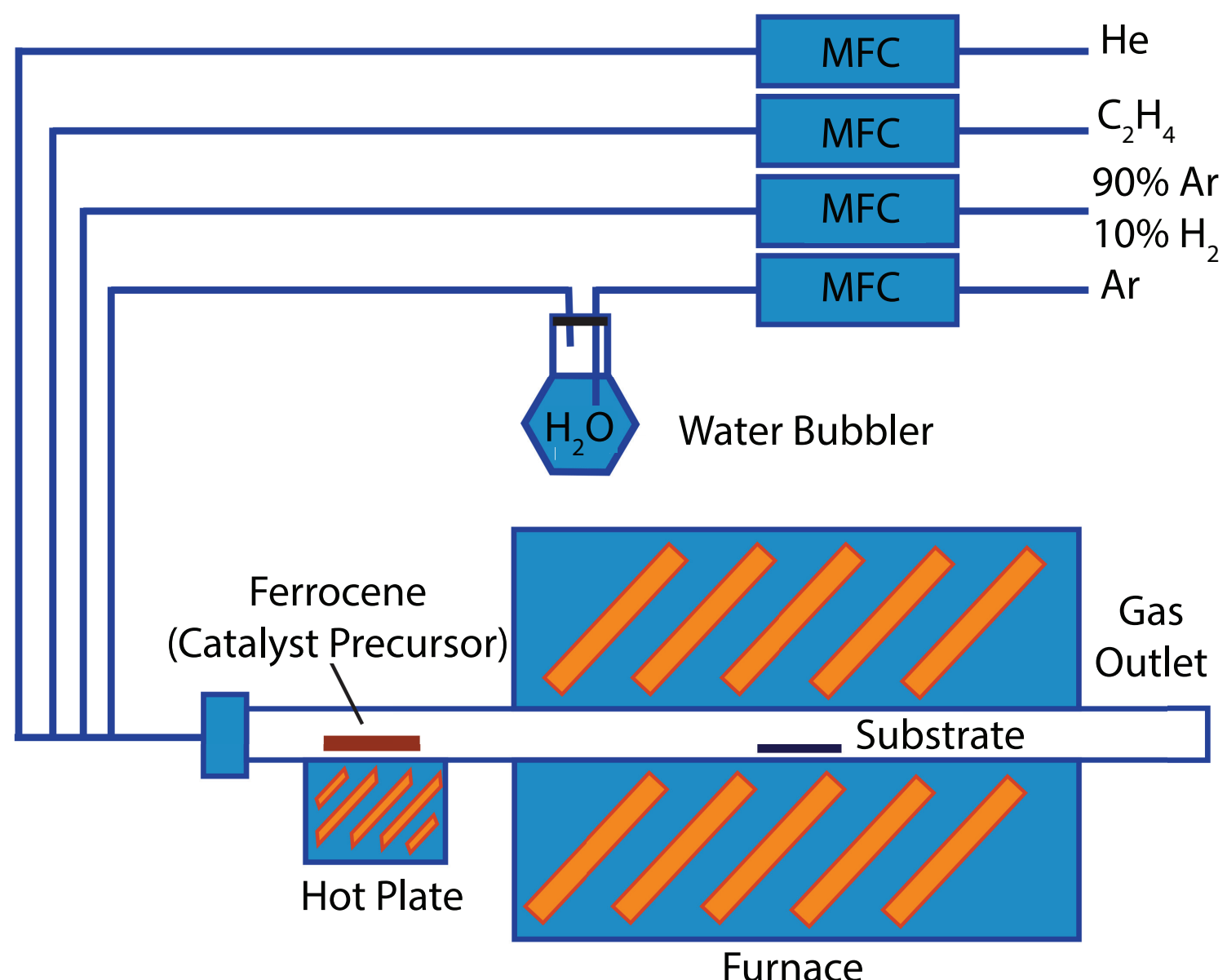

FiguRE 6.1: VACNT chemical vapor deposition growth chamber. The $100 \%$ argon source is tuned to vary water vapor transported into the system, altering the sample properties. The flow rate of the argon hydrogen mix is varied which also serves to tune the intrinsic properties of the sample. Ethylene serves as the carbon source material, and sublimated ferrocene produces iron catalyst particles.

introducing the 9:1 ratio of argon to hydrogen at $400 \mathrm{sccm}$ and the helium at 600 sccm to ensure the environment is inert and to prevent oxidation of the carbon. The hot plate depicted in Figure 6.1 sublimates the ferrocene at $180^{\circ} \mathrm{C}$, introducing iron catalyst particles into the growth chamber. The furnace is heated to $750^{\circ} \mathrm{C}$ where the catalyst precursor is deposited directly onto the substrate. After 20 minutes of equilibration, ethylene is introduced into the the system and nucleation of CNTs occurs. Ethylene decomposes and reacts with the deposited iron catalyst particles. With the growth conditions carefully maintained the precipitated graphite carbon 

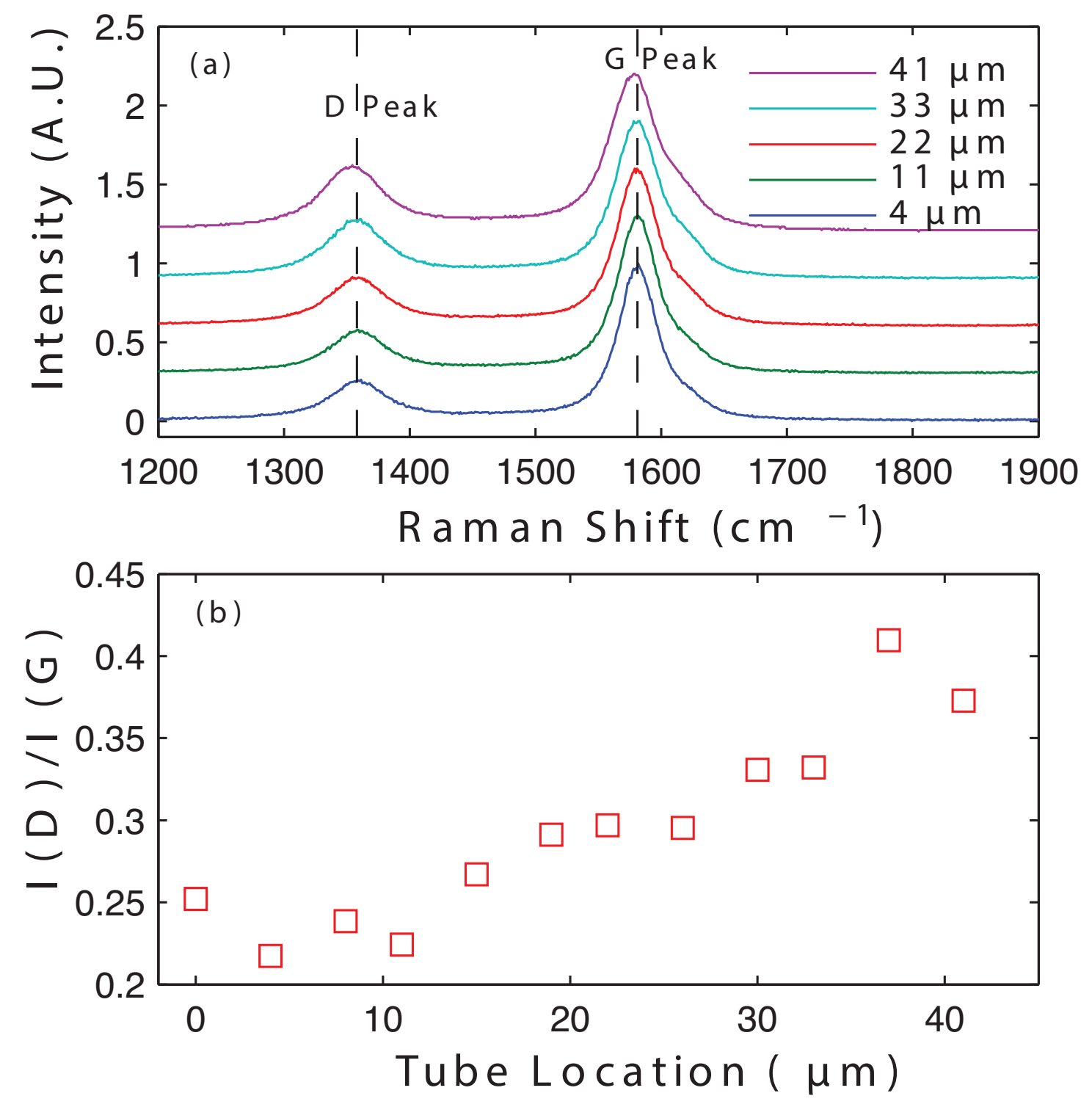

Figure 6.2: (a) Five representative Raman spectra of CNT array Sample D. (b) Ratio of the D and $G$ peak intensities versus position along the CNT array in Sample D. As we move up the array $(1$ to $41 \mu \mathrm{m})$ the ratio increases indicating increased disorder in the tubes.

forms cylindrical CNTs which remained adhered to the substrate through a Van der Waals adhesion.[208] Van der Waals forces between individual CNTs force the densely packed tubes to grow vertically.[209] Ethelyne flow, and therefore CNT growth, continues for 10 minutes.

Five samples, labeled $\mathrm{B}, \mathrm{C}, \mathrm{D}, \mathrm{E}$, and $\mathrm{F}$, were fabricated with water assisted 
growth without hydrogen present during growth. Argon is piped into an encapsulated beaker filled with water. Built-up gas pressure forces the water vapor into the tube furnace. The flow rate of argon into the the beaker is systematically increased for each sample: 100, 200, 400, 600 and $800 \mathrm{sccm}$ for sample B, C, D, E, and F respectively. Previous investigations have suggested that water vapor can selectively remove amorphous carbon deposited on top of the graphene-catalyst interface, enhancing catalyst activation during synthesis.[207] Helium is again fed into the tube furnace to maintain an inert atmosphere. The argon/hydrogen mixture flows into the chamber at $400 \mathrm{sccm}$ until the ethylene is introduced, at which time it is shut off and the helium's flow rate increased to $900 \mathrm{sccm}$. The final two samples, labeled $\mathrm{G}$ and $\mathrm{H}$, were fabricated with water assistance as well as increased argon/hydrogen mixture flow rate. For these two samples, argon flows through the water bubbler at $200 \mathrm{sccm}$. The flow rate of the argon/hydrogen mix is maintained at 800 sccm for sample $\mathrm{G}$ and 1200 sccm for sample $\mathrm{H}$ for the duration of the growth time.

\subsection{Structural characterization}

Raman spectroscopy was used to characterize the microstructure of our CNT arrays. The main features of the Raman spectra that we used are the two peaks at $\sim 1350$ and $\sim 1600 \mathrm{~cm}^{-1}$. The peak located at $\sim 1600 \mathrm{~cm}^{-1}$ corresponds to $\mathrm{sp}^{2}$ graphite like $\Gamma$ point vibrations and is thus named the $\mathrm{G}$ or graphite peak. The lower frequency peak is from modes at the $K$ point of the Brillioun zone of graphite which in a perfect graphite crystal are disallowed Raman active modes. However, disorder relaxes the selection rules giving rise to the observed peak at $\sim 1350 \mathrm{~cm}^{-1}$ and thus the name D peak or disorder peak. Naturally, this leads to the relative intensities of these peaks being used to quantify disorder in carbon systems. [180, 181]

Our Raman spectra were collected using a Renishaw InVia Raman microscope. 


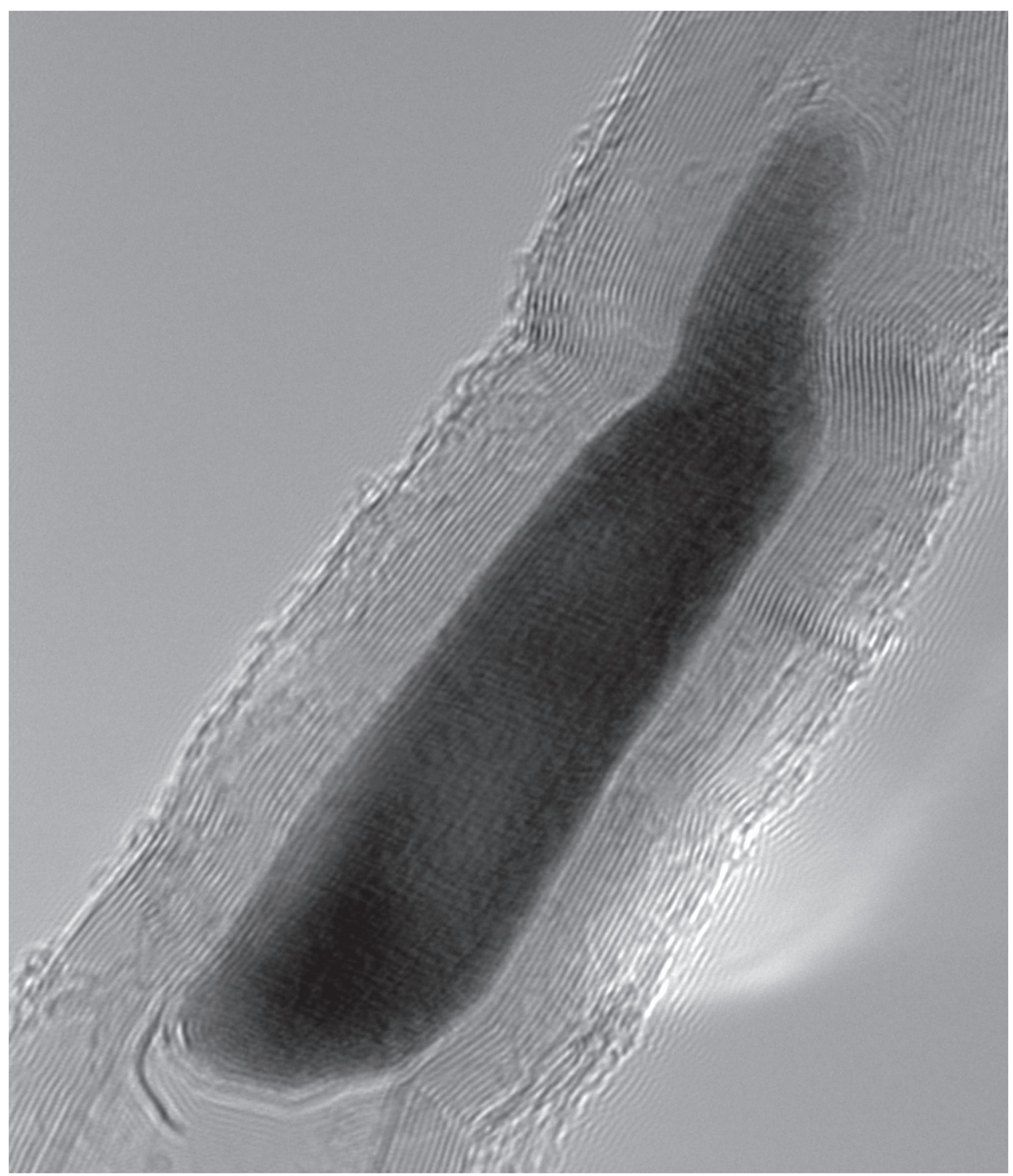

FIgURE 6.3: Transmission electron microscope image of a portion of a carbon nanotube from Sample C. At the widest point the CNT diameter is $25 \mathrm{~nm}$. Within the nanotube is a piece of iron with a $12 \mathrm{~nm}$ diameter at the widest point. It is apparent that the catalyst particle, a remnant from the growth process, alters the nanotubes inner diameter and outer diameter.

The system utilizes the $488 \mathrm{~nm}$ line of an Ar ion laser in $180^{\circ}$ back scattering geometry and a $3000 \mathrm{~g} / \mathrm{mm}$ grating. The sample was irradiated with a $0.1 \mathrm{~mW}$ laser 
power through a 50x $0.75 \mathrm{NA}$ objective yielding a spot diameter of $900 \mathrm{~nm}$. Before spectra were taken the sample arrays were first cleaved by chipping one edge of the quartz substrate to expose the center of the arrays. Ten spectra were collected, 360 second acquisition times, on each sample at 10 different locations of the sample spaced approximately $5 \mu \mathrm{m}$ apart along the length of the tube array. Five

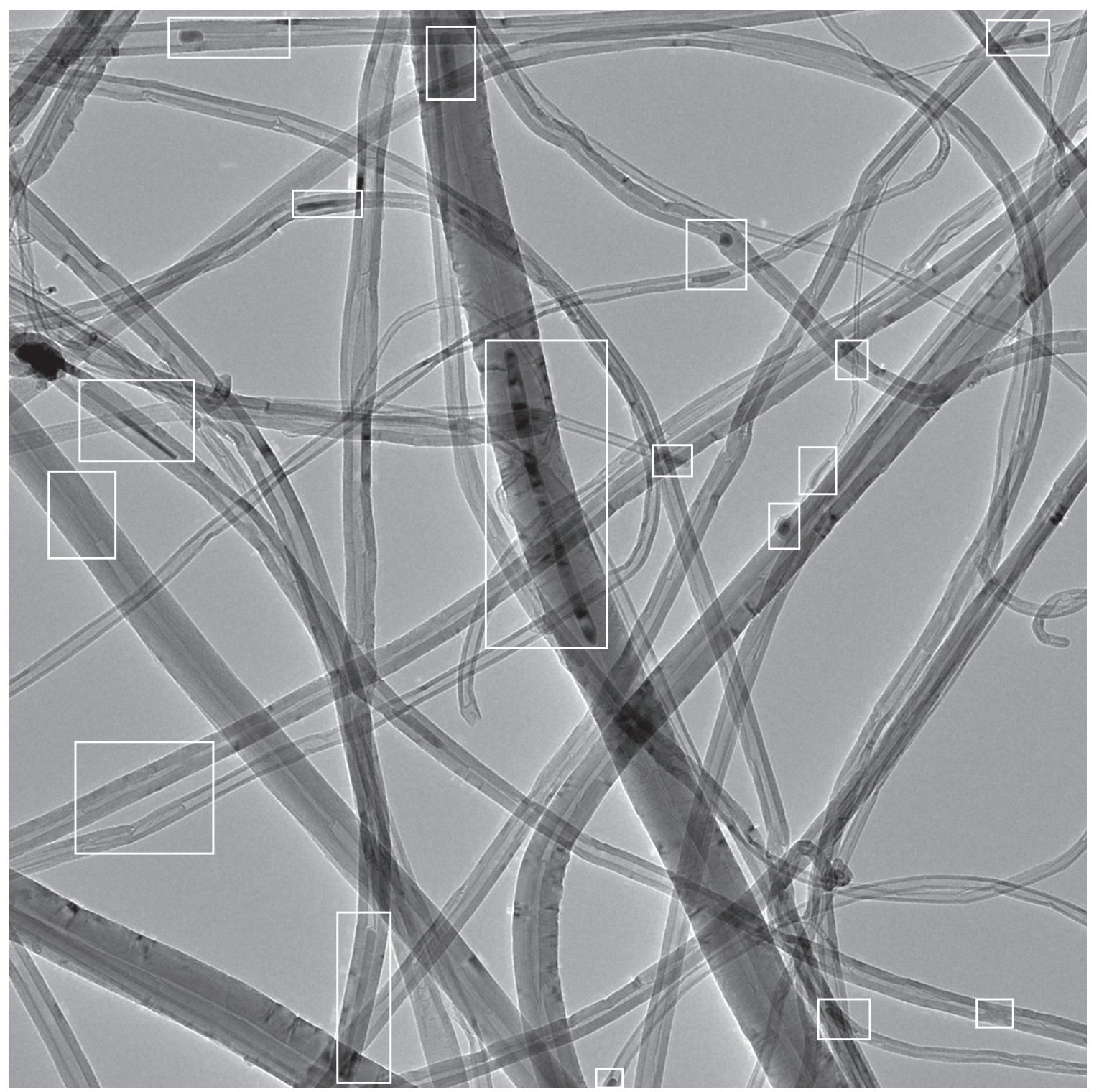

Figure 6.4: A $1 \mu \mathrm{m}$ by $1 \mu \mathrm{m}$ TEM image of several carbon nanotubes from Sample C. Throughout the many multi walled tubes, the dark catalyst particles are regularly present (highlighted by white rectangles), often significantly altering the tubes' features.

representative spectra of Sample D are plotted in Figure 6.2a. The most obvious 
changes are seen in the line width of the $G$ peak and in the relative size of the shoulder on the $\mathrm{G}$ peak as we move from the base of the CNT array, $0 \mu \mathrm{m}$, to the tips, $41 \mu \mathrm{m}$, both of which indicate an increase in disorder. To extract qualitative data, each spectrum was fitted with a three Voigt function fit, one function to the D peak, one to the $\mathrm{G}$ peak and one to the $\mathrm{G}^{+}$peak near $1620 \mathrm{~cm}^{-1}$. The ratio of the $\mathrm{D}$ and $\mathrm{G}$ peak intensities is plotted in Figure 6.2b. The $\mathrm{I}(\mathrm{D}) / \mathrm{I}(\mathrm{G})$ ratio increases from the bottom to the top of the tube indicating a decreasing order at the top of the tubes. However, at the very base of the tubes we observed a large degree of disorder (not shown here). This is because the tubes grow from the catalyst iron particles which are deposited on the substrate and so the carbon on the substrate has not yet formed into ordered tubes. The result indicates that the CNT arrays are not homogeneous throughout as initially thought. Very similar results were found for all other samples investigated in this study. Some samples had slightly smaller or larger changes in $\mathrm{I}(\mathrm{D}) / \mathrm{I}(\mathrm{G})$ from base to tip but with no discernible trend with changing growth parameters.

To investigate the origin of this change, the CNTs are removed from the substrate and observed using transmission electron microscopy. The TEM images confirm the CNTs in our samples are composed of multi-walled carbon nanotubes, with most tubes exhibiting dozens of concentric graphene layers. These images also display the likely cause of the trending $I_{D}$ to $I_{G}$ ratio: catalyst particles remaining in the center of the nanotubes. Figure 6.3 displays a TEM image focused on an iron catalyst particle within a multi walled carbon nanotube. It is apparent that the presence of this particle influences the shape, inner diameter and outer diameter of the CNT. Figure 6.4 displays several CNTs from Sample A. Even in this relatively small image of $1 \mu \mathrm{m}$ by $1 \mu \mathrm{m}$, at least one and often several catalyst particles can be identified in each CNT. The catalyst material still present varies significantly in shape. However in all cases it alters the nature of the CNT that contains it. The most pronounced effect is a change in number of concentric carbon tubes on either side of the catalyst 
particle.

TABle 6.1: Sample Summary (For each sample the variable fabrication parameters are displayed. The flow rate of argon is indicative of the amount of water vapor present during growth. The flow rate of the 9:1 ratio of argon and hydrogen has two stages, the flow prior to ethylene's introduction into the system, and the flow rate once this carbon source has been introduced. The last two columns display the best first thermal conductivity and volumetric heat capacity from $3 \omega$ data which have an uncertainty of $13 \%$ and $17 \%$ respectively.)

\begin{tabular}{lcccc}
\hline \hline Sample & $\begin{array}{c}\mathrm{Ar}\left(\mathrm{H}_{2} \mathrm{O}\right) \\
(\mathrm{sccm})\end{array}$ & $\begin{array}{c}\mathrm{Ar} / \mathrm{H}_{2} \mathrm{FR} \\
(\mathrm{sccm})\end{array}$ & $\begin{array}{c}\text { Conductivity } \\
(\mathrm{W} / \mathrm{m} \mathrm{K})\end{array}$ & $\begin{array}{c}\text { Heat Capacity } \\
\left(\mathrm{kJ} / \mathrm{m}^{3} \mathrm{~K}\right)\end{array}$ \\
\hline $\mathrm{A}$ & 0 & $400 \rightarrow 0$ & 49 & 530 \\
$\mathrm{~B}$ & 100 & $400 \rightarrow 0$ & 64 & 590 \\
$\mathrm{C}$ & 200 & $400 \rightarrow 0$ & 62 & 560 \\
$\mathrm{D}$ & 400 & $400 \rightarrow 0$ & 55 & 590 \\
$\mathrm{E}$ & 600 & $400 \rightarrow 0$ & 58 & 550 \\
$\mathrm{~F}$ & 800 & $400 \rightarrow 0$ & 69 & 570 \\
$\mathrm{G}$ & 200 & $800 \rightarrow 800$ & 74 & 550 \\
$\mathrm{H}$ & 200 & $1200 \rightarrow 1200$ & 79 & 560 \\
\hline \hline
\end{tabular}

\subsection{Thermal characterization and discussion}

The thermal conductivity and volumetric heat capacity of the samples are characterized using a $3 \omega$ technique. $\mathrm{Hu}$ and coauthors initially developed a modification to measure VACNT with $3 \omega .[90]$ We have previously built on this idea to measure any compliant film while optimizing sensitivity of the experiment to the parameters of interest by avoiding a one dimensional heat flow assumption within the substrate. [4] The experimental circuit used is similar to that first employed by Cahill and Pohl which was discussed in Chapter 3.[55] Our heating wire is fabricated on a hard baked polymer layer which is both electrically and thermally insulative. $\mathrm{A} \mathrm{SiO}_{2}$ layer is deposited above the heating wire to prevent electrical current from leaking into the sample. The VACNT is then pressed onto this passivation layer directly above the heating wire. The thermal diffusion equation is then solved for this stratified medium. $[4,87]$ The thermophysical properties of the 
rest of the system are independently verified, leaving the VACNT thermal conductivity, volumetric heat capacity, and the contact resistance between the passivation layer and the sample as the only fitting parameters.

Initial thermal conductivity experiments failed when the VACNT pierced the passivation layer and came into electrical contact with the heating wire. For this reason we increased the size of the passivation layer by an order of magnitude from $25 \mathrm{~nm}$ to $240 \mathrm{~nm}$. This increase does not significantly diminish the sensitivity of the experiment to the parameters of interest.[4]. Five independent measurements were performed on each sample with the loading pressure varied from $50 \mathrm{kPA}$ to 200 $\mathrm{kPA}$. All data was collected at room temperature and under atmospheric pressure.

Over the pressure range tested, best fit contact resistances between the sample and passivation layer varied between $1.8 \times 10^{-4}$ and $5 \times 10^{-5} \mathrm{~J} / \mathrm{m}^{3} \mathrm{~K}$ with greater pressures always resulting in lower contact resistance. For all samples the best fit thermal conductivity remained within $6 \%$ of the mean values displayed in Table 6.1 . The best fit volumetric heat capacity remained within $7 \%$ of the mean value. Over the pressure range evaluated, neither the thermal conductivity nor heat capacity of the samples systematically varied with increased applied pressure. We infer for these pressure ranges the intrinsic thermal properties of the VACNT are independent of loading pressure.

Table 6.1 displays the results for the eight samples tested. It is apparent that the addition of water improved the sample thermal conductivity slightly beyond the estimated error associated with the measure of $13 \%$ in most cases. However no clear trend exists with increasing flow rate through the bubbler. By increasing the hydrogen content in the growth environment, the sample thermal conductivity has seemingly been further increased. The cause for this increase is not fully clear from our data. Raman measurements revealed no significant change in the ordering or purity of the carbon between samples. While the water assisted samples appear to be slightly more dense as indicated by the measured volumetric heat capacity, the change is well within 
the measurement's uncertainty.

Whether the cause of the changing thermal conductivity between the samples is changing density, carbon purity, or due to some other effect, the influence is small compared the reduction in thermal transport caused by the catalyst particles in the center of the tube. Aliev and coauthors have suggested that at the highest theoretical packing density VACNTs of multi walled tubes can achieve a thermal conductivity near $600 \mathrm{~W} / \mathrm{m}$ K.[206] For samples grown through similar CVD processes, our work indicates that catalyst particles existing as a permanent feature throughout the VACNT reduce the tube fill fraction by increasing each tube's inner diameters which limits the sample density.[210] Furthermore, structural changes along the axis of the tube inherently causes phonon scattering ensuring the individual CNTs never achieve their quasi-ballistic potential. Rather than having a phonon mean free path comparable to the individual carbon nanotube's length, these defects increase the frequency of phonon scattering events and limit thermal transport. In order to maximize waste heat removal carbon nanotube systems, effort should be taken to understand and account for inhomogeneity in the system.

\subsection{Summary}

VACNTs where generated via chemical vapor deposition with the presence of hydrogen and water vapor in the growth chamber varied between samples. The CNT structure did not appreciably vary between samples as indicated by Raman spectroscopy and TEM images. However, the CNTs did change structure along the height of the sample due to the presence of catalyst particles throughout the array. The measured thermal conductivity of the samples varied from 49 to $79 \mathrm{~W} / \mathrm{m} \mathrm{K}$ with the presence of water vapor as well as hydrogen during growth seeming to increase the resulting thermal transport of the system. The presence of iron particles within

the individual CNTs of the samples produced for this study, prevents the VACNTs 
from achieving the high thermal conductivity necessary to serve as a TIM. 


\section{Chapter 7}

\section{Summary and Future Studies}

\subsection{Summary of conclusions}

Unique heterogenous material systems have aided in the advancement of devices with microscale dimensions. Understanding how to optimize thermal transport in such systems is critical to the continued advancement of such devices. It can be challenging to predict thermal transport in heterogenous systems. This difficulty is increased as feature sizes approach the thermal energy carrier's mean free path. Additionally, fabricating a heterogeneous material system of tunable feature sizes and intrinsic properties is not always achievable. In Chapter 1 nanotechnology applications motivating control of thermal conductance in heterogenous systems were discussed. Chapter 2 highlighted the fundamental interactions dictating thermal transport in nanoscale, heterogenous systems.

Chapter 3 introduced the $3 \omega$ technique and its utilization in measuring properties of the systems in this work. The heat diffusion equation can be solved for a material system with any number of planar layers for either single or bidirectional heat flow. A novel bidirectional $3 \omega$ experimental apparatus presented in this chapter utilizes the solution to heat flowing above and below the heating source and is shown capable of of enabling measurement of the properties of systems that are difficult or impossible to 
measure with traditional $3 \omega$ techniques. The successful experimental implementation of the traditional and bidirectional implementations of $3 \omega$ are confirmed with samples of known thermophysical properties which gives confidence to later studies of more novel material systems.

Chapters 4-6 each detailed a study on a separate heterogenous material system. Chapter 4 demonstrated the applicability of thin film silica aerogels as thermal insulation in microscale devices. The first objective of this chapter was to develop and integrate an aerogel film into a wafer with sufficient thermal insulation to prove a suitable alternative to current industrial practices. To this end, several thin film aerogels were fabricated under differing conditions. A process for depositing and etching metal onto the samples was developed. Sample thermal conductivity measurements showed aerogel films to be a potentially superior alternative to wafer back etching to create thermal insulation. Following this study, a large set of samples with varying fabrication parameters was generated to provide insight into thermal transport within thin film aerogels. These studies showed the aerogel microstructure can increase the specific heat capacity of silica aerogels above that of monolithic amorphous $\mathrm{SiO}_{2}$. Thermal conductivity results aligned with previous works demonstrating high porosities lead to low thermal conductivities. Additionally, samples with smaller pores and more fractal interconnects correlated with higher porosities in these thin film samples. While the fractal nature of the aerogel system may have some direct role in thermal transport, the fractal network has a greater indirect effect on thermal conductivity as a key attribute to obtaining highly porous thin film aerogels.

Chapter 5 utilized the bidirectional $3 \omega$ apparatus to examine the attributes which contribute to thermal transport in turbostratic carbon nanofiber networks. To isolate the contributions of system parameters influencing the effective thermal conductivity, the fiber network and testing environment were systematically varied. Four major knobs were tuned: applied loading pressure, atmospheric or vacuum 
pressure, heat treatment temperature of the samples, and uncompressed sample density. Tuning these parameters allowed for the identification of the roles of intrinsic fiber conductivity, gas conductivity, fiber orientation, fiber density, and fiber contact conductance in thermal transport of the system. Predictive thermal modeling was then applied taking into account these attributes. This modeling agreed with measured results which gives confidence to its utility in a broad range of scenarios.

Chapter 6 examined the use of vertically aligned carbon nanotube arrays to aid in removing heat as a thermal interface material. In order to identify and overcome obstacles preventing carbon nanotube arrays from achieving their theorized thermal transport potential, samples fabricated under systematically varied growth conditions were analyzed with Raman spectroscopy and transmission electron microscopy. It was shown that iron catalyst particles remaining within the tubes fundamentally alter the shape and quality of each CNT. Again the bidirectional $3 \omega$ modification was utilized to determined the thermal conductivity of this otherwise difficult to characterize system. Tuning of hydrogen and water vapor concentrations during fabrication affected the thermal conductivity of the system. However, the presence of the iron particles inside the CNTs is significantly hindering thermal transport and decreases the viability of using CNT arrays as thermal interface materials when fabricated through chemical vapor deposition.

Information gathered from these studies furthers the understanding of thermal energy transport in heterogenous material systems. By controlling fabrication processes to tune the nanostructure of material systems, structural and thermal characterization have provided insight into optimizing thermal transport in microscale applications. A modification of the $3 \omega$ technique was developed and proved critical in obtaining these findings. Experimental findings over a broad range of samples of each material system studied have been presented. These results have advanced the knowledge of thermal transport in each system. Ideally such insight 
can be used as an additional step for others to further advance the understanding of such systems and implement superior thermal control into microscale devices.

\subsection{Suggested future studies}

At the end of a study, it seems impossible to avoid looking back at all the paths not taken with perfect hindsight. With this knowledge in mind, these are such studies that now seem apparent for advancing the work presented:

- Error reduction in $3 \omega$ experiments through multiple wire widths. One challenge of thermal characterization of microscale material systems is the inherently large uncertainty. Thermal conductivity and thermal diffusivity are relatively complex terms to quantify. Chapter 3 opened discussions on changing the width of the heater wire to tune sensitivity to the parameters of interest. However, if data was taken on wires of perhaps four unique widths, then (depending on the properties and thickness of the film of interest) four unique sensitivity ranges may exist effectively allowing a fit of four unique experiments. Using multiple line widths would not increase the time spent fabricating on the sample. Minimizing experimental uncertainty would aid in observing more nuanced phenomena altering thermal transport in thin film systems.

- Thermal transport in crystalline, fractal aerogels. As highlighted in Chapter 4, the range of materials used as the backbone in aerogel systems has expanded drastically. Some of these systems maintain the fractal nature shown in silica aerogels while the atoms arrange themselves in a crystalline network. In the amorphous silica chains studied in Chapter 4, uncoupled vibrations of atoms dominate thermal transport making it difficult to see any unique effects microstructuring may have on the thermal conductivity of the system. However in a crystalline system where the energy carrier mean free path can 
be hundreds of nanometers, the role of fractons may be significantly more evident.

- Optimization of thin film silica aerogel pore size via real time SAXS measurements during the condensation, drying, and heat treatment processes. SAXS has been widely used to monitor the evolution of sol-gels and other two phase systems during the chemical reaction. Two step acid-base condensation and hydrolysis, as used in Chapter 4, can be highly dependent on the timing and density of each component. By monitoring the evolution of the sol during this reaction, insight can be gathered to further optimize pore size. Similarly SAXS measurements during ambient drying and heat treatment of the sample can provide insight into the evolution of the porous structure, which would aid in identifying the ideal fabrication conditions to correlate with thermal measurements.

- The effect of strain on thermal transport in carbon fiber networks. The bidirectional experiment discussed in Chapter 3, with a demonstrated utility in measuring carbon fibers demonstrated in Chapter 5, allows for measurements under a moderate range of applied pressures. This resulted in only a limited range of average fiber orientations being observable during testing (from randomly oriented to nearly all perpendicular). If however, the system could be studied as the composite was being stretched, two key insights could be gained. First, thermal modeling could account for a broader range of fiber orientations and determine the applicability of the model implemented to the limit of fibers being oriented parallel to heat flow. Secondly, the effects on the system's effective conductance could be observed in the case of strained fibers - potentially altering both the intrinsic fiber conductivity as well as the fiber to fiber contact conductance.

- Thermal characterization of carbon nanotube arrays and the constituent 
individual nanotubes. The modification of the $3 \omega$ experimental technique proved successful in measuring VACNT composites. However, greater insight into the interactions of the system could be gained if the thermal conductivity of the individual nanotubes was known. As a step in characterizing the system in Chapter 6, nanotubes were removed from the sample substrate and examined using a TEM. Other researches have had success using modifications of $3 \omega$ to measure nanoscale wires and even isolated carbon nanotubes. If the nanotubes removed from the sample could be directly thermally characterized, then respective roles of intrinsic tube conductivity and the interactions between nanotubes in the sample would become more apparent. Ultimately, fabricators could better isolate growth conditions which optimize the thermal performance of CNT arrays.

It is my sincere hope that the novel contributions of the studies presented in this dissertation provide a stepping stone for minds greater than mine to stand upon and move forward in the pursuit of engineering and scientific objectives that will ultimately have a positive impact on society. 


\section{References}

[1] Cahill, D. G., and Pohl, R. Heat flow and lattice vibrations in glasses. Solid State Communications 70, 10 (1989), 927 - 930.

[2] Norris, P. M., And Shrinivasan, S. Aerogels: Unique material, fascinating properties and unlimited applications. In Annual Review of Heat Transfer, B. Prasad, Y. Jaluria, and G. Chen, Eds., vol. XIV. Begell House, Inc., 2005, pp. 385-408.

[3] Bauer, C. M. N. Fabrication of thin film silica aerogels for embedded thermal isolation in mems devices. Master's thesis, University of Virginia, 2011.

[4] Bauer, M. L., AND Norris, P. M. General bidirectional thermal characterization via the 3 technique. Review of Scientific Instruments 85, 6 (2014), - .

[5] Franklin, A. D., Luisier, M., Han, S.-J., Tulevski, G., Breslin, C. M., Gignac, L., Lundstrom, M. S., and Haensch, W. Sub-10 nm carbon nanotube transistor. Nano Letters 12, 2 (2012), 758-762.

[6] Chen, G. Nanoscale Energy Transport and Conversion: A Parallel Treatment of Electrons, Molecules, Phonons, and Photons. Oxford University Press, New York, New York, 2005.

[7] Cahill, D., Ford, W., Goodson, K., Mahan, G., Majumdar, A., Maris, H., Merlin, R., And Phillpot, S. Nanoscale thermal transport. Journal of Applied Physics 93, 2 (2003), 793 - 818.

[8] Estimated U.S. energy usage in 2013. Tech. rep., Lawrence Livermore National Laboratory, 2014.

[9] Hsiao, Y., Chang, W., and Chen, S. A mathematic model of thermoelectric module with applications on waste heat recovery from automobile engine. Energy 35, 3 (2010), 1447 - 1454.

[10] Saqr, K., Mansour, M., And Musa, M. Thermal design of automobile exhaust based thermoelectric generators: Objectives and challenges. International Journal of Automotive Technology 9, 2 (2008), 155-160. 
[11] BeLL, L. E. Cooling, heating, generating power, and recovering waste heat with thermoelectric systems. Science 321, 5895 (2008), 1457-1461.

[12] Yang, J., AND Caillat, T. Thermoelectric materials for space and automotive power generation. MRS Bulletin 31 (3 2006), 224-229.

[13] Ghoshal, U., and Schmidt, R. Refrigeration technologies for subambient temperature operation of computing systems. In Solid-State Circuits Conference, 2000. Digest of Technical Papers. ISSCC. 2000 IEEE International (Feb 2000), pp. 216-217.

[14] Minnich, A. J., Dresselhaus, M. S., Ren, Z. F., and Chen, G. Bulk nanostructured thermoelectric materials: current research and future prospects. Energy Environ. Sci. 2 (2009), 466-479.

[15] Poudel, B., Hao, Q., Ma, Y., Lan, Y., Minnich, A., Yu, B., Yan, X., Wang, D., Muto, A., Vashaee, D., Chen, X., Liu, J., Dresselhaus, M. S., Chen, G., AND Ren, Z. High-thermoelectric performance of nanostructured bismuth antimony telluride bulk alloys. Science 320, 5876 (2008), 634-638.

[16] Dresselhaus, M., Chen, G., Tang, M., Yang, R., Lee, H., Wang, D., Ren, Z., Fleurial, J.-P., And Gogna, P. New directions for lowdimensional thermoelectric materials. Advanced Materials 19, 8 (2007), 10431053.

[17] Ganguly, S., Zhou, C., Morelli, D., Sakamoto, J., And Brock, S. L. Synthesis and characterization of telluride aerogels: Effect of gelation on thermoelectric performance of bi2te 3 and bi2-xsbxte3 nanostructures. The Journal of Physical Chemistry C 116, 33 (2012), 17431-17439.

[18] Madani, M., Lankireddy, D., and Tzeng, N.-F. Gaseous sensors with area- and energy-efficient microhotplates through silica aerogel for heat insulation. In International Conference on Microelectronics (Piscataway, NJ, USA, 2009), pp. $402-5$.

[19] Moore, G. E. Cramming more components cramming more components onto integrated cicuits. Electronics 38, 8 (1965).

[20] Prasher, R. Thermal interface materials: Historical perspective, status, and future directions. Proceedings of the IEEE 94, 8 (Aug 2006), 1571-1586.

[21] Ashcroft, N. W., And Mermin, N. D. Solid State Physics. Thomson Learning, 1976.

[22] Hopkins, P. E., Kaehr, B., Phinney, L. M., Koehler, T. P., Grillet, A. M., Dunphy, D., Garcia, F., And Brinker, C. J. Measuring the thermal conductivity of porous, transparent $\mathrm{SiO}_{2}$ films with time domain thermoreflectance. Journal of Heat Transfer 133, 6 (2011), 061601. 
[23] Park, J.-Y., Rosenblatt, S., Yaish, Y., Sazonova, V., Üstünel, H., Braig, S., Arias, T. A., Brouwer, P. W., and McEuen, P. L. Electronphonon scattering in metallic single-walled carbon nanotubes. Nano Letters 4, 3 (2004), 517-520.

[24] Klemens, P. Thermal conductivity and lattice vibrational modes. vol. 7 of Solid State Physics. Academic Press, 1958, pp. 1 - 98.

[25] Walker, C. T., And Pohl, R. O. Phonon scattering by point defects. Phys. Rev. 131 (Aug 1963), 1433-1442.

[26] Asheghi, M., Leung, Y. K., Wong, S. S., And Goodson, K. E. Phononboundary scattering in thin silicon layers. Applied Physics Letters 71, 13 (1997), 1798-1800.

[27] Einstein, A. Elementare betrachtungen über die thermische molekularbewegung in festen körpern. Annalen der Physik 340, 9 (1911), 679-694.

[28] Cahill, D. G., Watson, S. K., and Pohl, R. O. Lower limit to the thermal conductivity of disordered crystals. Phys. Rev. B 46, 10 (Sep 1992), 6131-6140.

[29] Majumdar, A. Lower limit of thermal conductivity: Diffusion versus localization. Microscale Thermophysical Engineering 2, 1 (1998), 5-9.

[30] Hopkins, P., And Piekos, E. Lower limit to phonon thermal conductivity of disordered, layered solids. Applied Physics Letters 94, 18 (2009), 181901.

[31] ALEXANDER, S., AND ORBACH, R. Density of states on fractals: "fractons". J. Physique Lett. 43, 17 (1982), 625-631.

[32] Alexander, S., Laermans, C., Orbach, R., and Rosenberg, H. M. Fracton interpretation of vibrational properties of cross-linked polymers, glasses, and irradiated quartz. Phys. Rev. B 28 (Oct 1983), 4615-4619.

[33] Alexander, S., Entin-Wohlman, O., and Orbach, R. Phonon-fracton anharmonic interactions: The thermal conductivity of amorphous materials. Phys. Rev. B 34 (Aug 1986), 2726-2734.

[34] Turcotte, D., And Oxburgh, A. Finite amplitude convective cells and continental drift. Journal of Fluid Mechanics 28, Part 1 (1967), 29 - 42.

[35] Clyne, T., Golosnoy, I., Tan, J., and Markaki, A. Porous materials for thermal management under extreme conditions. Philos. Trans. R. Soc. Lond. A, Math. Phys. Eng. Sci. (UK) 364, 1838 (2006/01/15), 125 - 46.

[36] Kaganer, M. G. Thermal Insulation in Cryogenic Engineering. Israel Program for Scientific Translations, 1969. 
[37] Tien, C., And Lienhard, J. Statistical Thermodynamics. HRW series in mechanical engineering. Taylor \& Francis, 1979.

[38] Kennard, E. Kinetic theory of gases: with an introduction to statistical mechanics. International series in pure and applied physics. McGraw-Hill, 1938.

[39] RaEd, K., And Gross, U. Modeling of influence of gas atmosphere and pore-size distribution on the effective thermal conductivity of knudsen and nonknudsen porous materials. International Journal of Thermophysics 30, 4 (2009), $1343-1356$.

[40] Nan, C.-W., Birringer, R., Clarke, D. R., And Gleiter, H. Effective thermal conductivity of particulate composites with interfacial thermal resistance. Journal of Applied Physics 81, 10 (1997), 6692 - 6699.

[41] Minnich, A., And Chen, G. Modified effective medium formulation for the thermal conductivity of nanocomposites. Applied Physics Letters 91, 7 (2007), $073105-1$.

[42] Poon, S. J., Petersen, A. S., And Wu, D. Thermal conductivity of core-shell nanocomposites for enhancing thermoelectric performance. Applied Physics Letters 102, 17 (2013).

[43] Kapitza, P. L. Heat transfer and superfluidity of helium ii. Physical Review 60, 4 (Aug 1941), 354-355.

[44] POLlACK, G. L. Kapitza resistance. Rev. Mod. Phys. 41 (Jan 1969), 48-81.

[45] Chen, G. Thermal conductivity and ballistic-phonon transport in the crossplane direction of superlattices. Physical Review B 57, 23 (1998), 14958 - 14973.

[46] Swartz, E., And Pohl, R. Thermal resistance at interfaces. Appl. Phys. Lett. (USA) 51, $26(1987 / 12 / 28), 2200-2$.

[47] Son, Y., Pal, S. K., Borca-Tasciuc, T., AJayan, P. M., And SIEGEL, R. W. Thermal resistance of the native interface between vertically aligned multiwalled carbon nanotube arrays and their sio2 /si substrate. Journal of Applied Physics 103, 2 (2008). Mechanical contact;Nanoconstrictions;Photothermoelectric technique;.

[48] Norris, P., AND Hopkins, P. Examining interfacial diffuse phonon scattering through transient thermoreflectance measurements of thermal boundary conductance. Journal of Heat Transfer 131, 4 (2009), 043207.

[49] Hopkins, P., AND Norris, P. Relative contributions of inelastic and elastic diffuse phonon scattering to thermal boundary conductance across solid interfaces. Journal of Heat Transfer 131, 2 (2009), 022402. 
[50] English, T. S., Duda, J. C., Smoyer, J. L., Jordan, D. A., Norris, P. M., AND Zhigilei, L. V. Enhancing and tuning phonon transport at vibrationally mismatched solid-solid interfaces. Phys. Rev. B 85 (Jan 2012), 035438 .

[51] Kaviany, M. Principles of heat transfer in porous media. Springer-Verlag, 1991.

[52] Kaviany, M. Heat Transfer Physics. Cambridge University Press, 2014.

[53] Fish, M. C. Application of the $3 \omega$ method for the determination of aerogl thin film thermal conductivity. Master's thesis, University of Virginia, 2010.

[54] Corbino, O. M. Periodic resistance changes of fine metal threads, which are brought together by alternating streams as well as deduction of their thermo characteristics at high temperatures. Physikalische Zeitschrift 12 (1911), 292295.

[55] Cahill, D. G., And Pohl, R. O. Thermal conductivity of amorphous solids above the plateau. Physical Review B 35, 8 (1987), 4067-4073.

[56] O.M.Corbino. Thermal oscillations in lamps of thin fibers with alternating current flowing through them and the resulting effect on the rectifier as a result of the presence of even-numbered harmonics. Physikalische Zeitschrift 11 (1910), 413-417.

[57] Kraftmakner, Y. Modulation Calorimetry: Theory and Applications. Springer, 2004.

[58] Filippov, L. Methods of simultaneous measurement of heat conductivity, heat capacity and thermal diffusivity of solid and liquid metals at high temperatures. International Journal of Heat and Mass Transfer 9, 7 (1966), 681 - 691.

[59] Rosenthal, L. A. Thermal response of bridgewires used in electroexplosive devices. Review of Scientific Instruments 32, 9 (1961), 1033-1036.

[60] Holland, L. R. Physical properties of titanium. iii. the specific heat. Journal of Applied Physics 34, 8 (1963), 2350-2357.

[61] Birge, N. O., And Nagel, S. R. Specific-heat spectroscopy of the glass transition. Phys. Rev. Lett. 54 (Jun 1985), 2674-2677.

[62] Birge, N. O., And Nagel, S. R. Wide-frequency specific heat spectrometer. Review of Scientific Instruments 58, 8 (1987), 1464-1470.

[63] Jung, D. H., Kwon, T. W., Bae, D. J., Moon, I. K., And Jeong, Y. H. Fully automated dynamic calorimeter. Measurement Science and Technology 3, 5 (1992), 475. 
[64] Moon, I. K., JeOng, Y. H., And Kwun, S. I. The 3 omega technique for measuring dynamic specific heat and thermal conductivity of a liquid or solid. Review of Scientific Instruments 67, 1 (1996), 29-35.

[65] Birge, N. O., Dixon, P. K., And Menon, N. Specific heat spectroscopy: Origins, status and applications of the $3 \omega$ method. Thermochimica Acta 304305, 0 (1997), 51 - 66. Temperature Modulated Calorimetry.

[66] Cahill, D. Thermal conductivity measurement from 30 to $750 \mathrm{k}$ : the $3 \omega$ method. Review of Scientific Instruments 61, 2 (1990), 802 - 808.

[67] Cahill, D. G. Erratum: Thermal conductivity measurement from 30 to 750 $\mathrm{k}$. the $3 \omega$ method (review of scientific instruments (1990) 61 (802)). Review of Scientific Instruments 73, 10 (2002), 3701 - 3701.

[68] Cahill, D., Katiyar, M., and Abelson, J. Thermal conductivity of a-Si:H thin films. Physical Review B 50, 9 (1994), 6077 - 6081.

[69] LeE, S.-M., And Cahill, D. Heat transport in thin dielectric films. J. Appl. Phys. (USA) 81, 6 (1997/03/15), $2590-5$.

[70] Yamane, T., Nagai, N., Katayama, S.-I., and Todoki, M. Measurement of thermal conductivity of silicon dioxide thin films using a $3 \omega$ method. Journal of Applied Physics 91, 12 (2002), 9772 -9776.

[71] Bullen, A., O’Hara, K., Cahill, D., Monteiro, O., And von Keudell, A. Thermal conductivity of amorphous carbon thin films. J. Appl. Phys. (USA) 88, 11 (2000/12/01), 6317 - 20. thermal conductivity;amorphous C thin films;effective-medium theory;plasma CVD films;a-C films;80 to $400 \mathrm{~K}$; ;.

[72] Hu, C., Morgen, M., Ho, P. S., Jain, A., Gill, W. N., Plawsky, J. L., AND WAYNER, JR., P. C. Thermal conductivity study of porous low-k dielectric materials. Applied Physics Letters 77, 1 (2000), 145 - 147.

[73] Delan, A., Rennau, M., Schulz, S., And Gessner, T. Thermal conductivity of ultra low-k dielectrics. Microelectronic Engineering 70, 2-4 (2003), $280-284$.

[74] Kim, J. H., Feldman, A., And Novotny, D. Application of the three omega thermal conductivity measurement method to a film on a substrate of finite thickness. J. Appl. Phys. (USA) 86, 7 (1999/10/01), 3959 - 63.

[75] Borca-Tasciuc, T., Kumar, A., And Chen, G. Data reduction in 3w method for thin-film thermal conductivity determination. Review of Scientific Instruments 72, 4 (2001), $2139-2147$.

[76] Olson, B. W., Graham, S., and Chen, K. A practical extension of the 3 omega method to multilayer structures. Review of Scientific Instruments 76, 5 (2005), 053901. 
[77] Tong, T., And Majumdar, A. Reexamining the 3-omega technique for thin film thermal characterization. Review of Scientific Instruments 7\%, 10 (2006), 104902 .

[78] Zong, Z.-X., Qiu, Z.-J., Zhang, S.-L., Streiter, R., And Liu, R. A generalized 3 omega method for extraction of thermal conductivity in thin films. Journal of Applied Physics 109, 6 (2011), 063502.

[79] Lu, L., Yi, W., And Zhang, D. L. 3 omega method for specific heat and thermal conductivity measurements. Review of Scientific Instruments 72, 7 (2001), 2996-3003.

[80] Choi, T. Y., Poulikakos, D., Tharian, J., And Sennhauser, U. Measurement of thermal conductivity of individual multiwalled carbon nanotubes by the 3-omega method. Applied Physics Letters 87, 1 (2005), 013108 .

[81] Hou, J., Wang, X., Vellelacheruvu, P., Guo, J., Liu, C., And Cheng, H.-M. Thermal characterization of single-wall carbon nanotube bundles using the self-heating 3 omega technique. Journal of Applied Physics 100, 12 (2006), 124314 .

[82] Hopkins, P., And Phinney, L. Thermal conductivity measurements on polycrystalline silicon microbridges using the 3 technique. Journal of Heat Transfer 131, 4 (2009), 043201.

[83] Vollebregt, S., Banerjee, S., Beenakker, K., And Ishihara, R. Thermal conductivity of low temperature grown vertical carbon nanotube bundles measured using the three-omega method. Applied Physics Letters 102, 19 (2013), 191909.

[84] Dames, C., And Chen, G. $1 \omega, 2 \omega$, and $3 \omega$ methods for measurements of thermal properties. Review of Scientific Instruments 76, 12 (2005), 124902.

[85] Tian, T. Anisotropic thermal property measurement of carbon-fiber/epoxy composit materials. PhD thesis, University of Nebraska, 2011.

[86] Carslaw, H. S., And Jaeger, J. C. Conduction of heat in solids, 2nd ed. Clarendon Press, Oxford, 1959.

[87] Feldman, A. Algorithm for solutions of the thermal diffusion equation in a stratified medium with a modulated heating source. High Temp. - High Press. (UK) 31, 3 (1999), $293-8$.

[88] Dames, C. Measuring the thermal conductivity of thin films: 3 omega and related electrothermal methods. In Annual Review of Heat Transfer (2013), G. Ghen, V. Prasad, and Y. Jolutia, Eds., vol. XVI, Begell home, Inc., pp. 749. 
[89] Smith, A. N., Hostetler, J. L., And Norris, P. M. Thermal boundary resistance measurements using a transient thermoreflectance technique. Microscale Thermophysical Engineering 4, 1 (2000), 51-60.

[90] Hu, X., Padilla, A., Xu, J., Fisher, T., and Goodson, K. 3-omega measurements of vertically oriented carbon nanotubes on silicon. Journal of Heat Transfer 128, 11 (2006), 1109 - 1113.

[91] Qiu, L., Tang, D. W., Zheng, X. H., and Su, G. P. The freestanding sensor-based 3 technique for measuring thermal conductivity of solids: Principle and examination. Review of Scientific Instruments 82, 4 (2011), 045106.

[92] Shigley, J., And Mischke, C. Mechanical Engineering Design. McGrawHill, 2008.

[93] Raudzis, C., Schatz, F., and Wharam, D. Extending the $3 \omega$ method for thin-film analysis to high frequencies. Journal of Applied Physics 93, 10 (2003), $6050-6055$.

[94] Touloukian, Y. Thermophysical Properties of Matter: Thermal conductivity : nonmetallic solids, vol. 2. IFI/Plenum, 1970.

[95] Touloukian, Y. Thermophysical Properties of Matter: Specific Heat : nonmetallic solids, vol. 5. IFI/Plenum, 1970.

[96] Wei, C., Zheng, X., Cahill, D. G., and Zhao, J.-C. Invited article: Micron resolution spatially resolved measurement of heat capacity using dualfrequency time-domain thermoreflectance. Review of Scientific Instruments 84, 7 (2013), - .

[97] Brinker, C. J., and Scherer, G. W. Sol-gel Science: the Physics and Chemistry of Sol-gel Processing. Academic Press, Boston, 1990.

[98] Dorchen, A. S., And AbBasi, M. Silica aerogel; synthesis, properties and characterization. Journal of Materials Processing Technology 199 (2008), 10 26 .

[99] Kistler, S. S. Coherent expanded aerogels and jellies. Nature 127, 3211 (1931), 741.

[100] Ruffner, J., Clem, P., Tuttle, B., Brinker, C., Sriram, C., And Bullington, J. Uncooled thin film infrared imaging device with aerogel thermal isolation: deposition and planarization techniques. Thin Solid Films (Switzerland) 332, 1-2 (1998), $356-61$.

[101] Burger, T., And Fricke, J. Aerogels: production, modification and applications. Berichte der Bunsengesellschaft/Physical Chemistry Chemical Physics 102, 11 (1998), 1523 - 1528. 
[102] Wei, T.-Y., Chen, C.-H., Chien, H.-C., Lu, S.-Y., And Hu, C.-C. A cost-effective supercapacitor material of ultrahigh specific capacitances: Spinel nickel cobaltite aerogels from an epoxide-driven sol-gel process. Advanced Materials 22, 3 (2010), 347-351.

[103] Jones, S. Aerogel: Space exploration applications. Journal of Sol-Gel Science and Technology 40, 2-3 (2006), 351-357.

[104] Zegaoui, O., Hoang-Van, C., And Karroua, M. Selective catalytic reduction of nitric oxide by propane over vanadia-titania aerogels. Applied Catalysis B: Environmental 9, 1-4 (1996), 211 - 227.

[105] Mizushima, Y., And Hori, M. Alumina aerogel for support of a methane combustion catalyst. Applied Catalysis A: General 88, 2 (1992), 137 - 148.

[106] Bryning, M., Milkie, D., Islam, M., Hough, L., Kikkawa, J., And Yodh, A. Carbon nanotube aerogels. Advanced Materials 19, 5 (2007), 661664.

[107] Bag, S., Arachchige, I. U., And Kanatzidis, M. G. Aerogels from metal chalcogenides and their emerging unique properties. J. Mater. Chem. 18 (2008), 3628-3632.

[108] Long, J. W., Logan, M. S., Rhodes, C. P., Carpenter, E. E., Stroud, R. M., And Rolison, D. R. Nanocrystalline iron oxide aerogels as mesoporous magnetic architectures. Journal of the American Chemical Society 126, 51 (2004), 16879-16889. PMID: 15612727.

[109] Berthon-Fabry, S., Langohr, D., Achard, P., Charrier, D., Djurado, D., ANd Ehrburger-Dolle, F. Anisotropic high-surface-area carbon aerogels. J. Non-Cryst. Solids (Netherlands) 350 (2004/12/15), 136 44.

[110] Gash, A. E., Tillotson, T. M., JR, J. H. S., Hrubesh, L. W., And Simpson, R. L. New sol-gel synthetic route to transition and main-group metal oxide aerogels using inorganic salt precursors. Journal of Non-Crystalline Solids 285, 1-3 (2001), $22-28$.

[111] Zeng, J., Stevens, P., Hunt, A., Grief, R., And Lee, D. Thinfilm-heater thermal conductivity apparatus and measurement of thermal conductivity of silica aerogel. International Journal of Heat and Mass Transfer 39, 11 (1996), $2311-2317$.

[112] Worsley, M. A., Pauzauskie, P. J., Olson, T. Y., Biener, J., Satcher, J. H., And Baumann, T. F. Synthesis of graphene aerogel with high electrical conductivity. Journal of the American Chemical Society 132, 40 (2010), 14067-14069. PMID: 20860374. 
[113] Devreux, F., Boilot, J. P., Chaput, F., And Sapoval, B. Nmr determination of the fractal dimension in silica aerogels. Phys. Rev. Lett. 65 (Jul 1990), 614-617.

[114] Emmerling, A., AND Fricke, J. Small angle scattering and the structure of aerogels. Journal of Non-Crystalline Solids 145, 1-3 (1992), 113 - 120. Aerogels;Nanoporous materials;Particle surface characterization;Small angle scattering;.

[115] Reidy, R. F., Allen, A. J., And Krueger, S. Small angle neutron scattering characterization of colloidal and fractal aerogels. Journal of NonCrystalline Solids 285, 1-3 (2001), 181 - 186.

[116] Vollet, D., Donatti, D., And Ibanez Ruiz, A. A saxs study of the nanostructural characteristics of teos-derived sonogels upon heat treatment up to 1100c. J. Non-Cryst. Solids (Netherlands) 306, 1 (2002/07/), $11-16$.

[117] Hsu, C.-H., Jeng, U.-S., Lee, H.-Y., Huang, C.-M., Liang, K., Windover, D., LU, T.-M., AND Jin, C. Structural study of a low dielectric thin film using $\mathrm{x}$-ray reflectivity and grazing incidence small angle x-ray scattering. Thin Solid Films 472, 1-2 (2005), 323 - 327.

[118] He, F., HE, X., AND Li, Y. Fractal characteristic of silica xerogels with different additives. In Nano/Micro Engineered and Molecular Systems, 2006. NEMS '06. 1st IEEE International Conference on (Jan 2006), pp. 1272-1275.

[119] Brinker, C., And Scherer, G. Sol gel glass: I. gelation and gel structure. Journal of Non-Crystalline Solids 70, 3 (1985), 301 - 322.

[120] Rao, A., Pajonk, G., And Rao, A. Effect of preparation conditions on the physical and hydrophobic properties of two step processed ambient pressure dried silica aerogels. Journal of Materials Science 40, 13 (2005), 3481-3489.

[121] Kistler, S., And Caldwell, A. Thermal conductivity of silica aerogel. Industrial and Engineering Chemistry 26, 6 (1934), 658 - 662.

[122] Kistler, S. S. The relation between heat conductivity and structure in silica aerogel. The Journal of Physical Chemistry 39, 1 (1934), 79-86.

[123] Posselt, D., Kuems, J. K., Bernasconi, A., Sleator, T., And Ott, H. R. The thermal conductivity of silica aerogel in the phonon, the fracton and the particle-mode regime. EPL (Europhysics Letters) 16, 1 (1991), 59.

[124] Scheuerpflug, P., Hauck, M., And Fricke, J. Thermal properties of silica aerogels between 1.4 and 330 k. Journal of Non-Crystalline Solids (1991), 196-201. Foreign technology;. 
[125] Fricke, J., And Emmerling, A. Aerogels. J. Am. Ceram. Soc. (USA) 75, 8 (1992/08/), 2027 - 36. aerogels;supercritical drying;porosity;Cerenkov detectors;passive solar energy usage;thermal superinsulations;heat-storage systems;catalytic substrates;acoustic impedance matching layers;high-quality glasses;fusion fuels;gas filters;radioluminescent light;

[126] Lu, X., Nilsson, O., Fricke, J., and Pekala, R. W. Thermal and electrical conductivity of monolithic carbon aerogels. Journal of Applied Physics 73, 2 (1993), 581-584.

[127] Lu, X., Caps, R., Fricke, J., Alviso, C., and Pekala, R. Correlation between structure and thermal conductivity of organic aerogels. Journal of Non-Crystalline Solids 188, 3 (1995), 226 - 234.

[128] Conroy, J., Hosticka, B., Davis, S., And Norris, P. Microscale thermal relaxation in silica aerogels. Microscale Thermophysical Engineering 2, 4 (1998), $228-229$.

[129] Hostler, S., Abramson, A., Gawryla, M., Bandi, S., and Schiraldi, D. Thermal conductivity of a clay-based aerogel. International Journal of Heat and Mass Transfer 52, 3-4 (2009), 665 - 669.

[130] Worsley, M., Satcher, J.H., J., And Baumann, T. Enhanced thermal transport in carbon aerogel nanocomposites containing double-walled carbon nanotubes. Journal of Applied Physics 105, 8 (2009), 084316.

[131] Schiffres, S. N., Kim, K. H., Hu, L., McGaughey, A. J. H., Islam, M. F., AND MAlen, J. A. Gas diffusion, energy transport, and thermal accommodation in single-walled carbon nanotube aerogels. Advanced Functional Materials (2012).

[132] Prakash, S., Brinker, C., And Hurd, A. Silica aerogel films at ambient pressure. Journal of Non-Crystalline Solids 190, 3 (1995), 264 - 275.

[133] Hrubesh, L., And Poco, J. Thin aerogel films for optical, thermal, acoustic and electronic applications. Journal of Non-Crystalline Solids (Netherlands) 188, 1-2 (1995), $46-53$.

[134] Prakash, S. S., Brinker, C., Hurd, A. J., And Rao, S. M. Silica aerogel films prepared at ambient pressure by using surface derivatization to induce reversible drying shrinkage. Nature 374, 6521 (1995), 439 - 443.

[135] Mathews, R. Improving insulation methods in new and existing fabrication processes. Master's thesis, Unversity of Virginia, 2012.

[136] Iler, R. The Chemistry of Silica: Solubility, Polymerization, Colloid and Surface Properties and Biochemistry of Silica. A Wiley-Interscience publication. Wiley, 1979. 
[137] GaO, G.-M., Xu, X.-C., Zou, H.-F., Ji, G.-J., And Gan, S.-C. Microstructural and physical properties of silica aerogels based on oil shale ash. Powder Technology 202, 1-3 (2010), 137 - 142.

[138] Shi, F., WANG, L., LiU, J., AND ZENG, M. Effect of heat treatment on silica aerogels prepared via ambient drying. J. Mater. Sci. Technol. (China) 23, 3 (2007//), 402 - 6. heat treatment;silica aerogels;ambient drying; ethanol/trimethylchlorosilane/heptane solution;pore water exchange;hydrophobicity;Brunauer- emmitt-teller surface area;morphology;chemical bonding state;scanning electron microscopy;differential temperature analysis;Fourier-transform infrared spectroscopy;150C to 500C; $\mathrm{SiO}_{\mathrm{i}} \mathrm{sub}_{\mathrm{i}} 2 \mathrm{i} / \mathrm{sub}_{\mathrm{i}}$ - Surface;temperature $150 \mathrm{C}$ to $500 \mathrm{C} ; \mathrm{SiO}_{j} \mathrm{sub}_{i} 2 \mathrm{i} /$ subi;

[139] Kratky, O., And Porod, G. Diffuse small-angle scattering of x-rays in colloid systems diffuse small-angle scattering of x-rays in colloid systems. $J$. Colloid Sci. 4 (1949), 35-70.

[140] Guinier, A., And Fournet, G. Small-angle scattering of X-rays. Structure of matter series. Wiley, 1955.

[141] Glatter, O., And Kratky, O. Small Angle X-ray Scattering. Academic Press, 1982.

[142] Daillant, J., And Gibaud, A. X-ray and Neutron Reflectivity: Principles and Applications. Lecture Notes in Physics. Springer, 2009.

[143] Freltoft, T., KJems, J., And Sinha, S. Power-law correlations and finite-size effects in silica particle aggregates studied by small-angle neutron scattering. Phys. Rev. B, Condens. Matter (USA) 33, 1 (1986/01/01), 269 - 75. $\mathrm{SiO}_{j} \mathrm{sub}_{2} 2_{\mathrm{i}}$ /sub¿;suspensions;finite-size effects;smallangle neutron scattering;fine silica particles;power-law correlations;scattering function;models;particle-particle correlation;fractal dimension;.

[144] Hsu, C.-H., Lee, H.-Y., Liang, K., Jeng, U.-S., Windover, D., Lu, T.-M., AND Jin, C. Grazing incidence small angle x-ray scattering study on low dielectric thin films. pp. $1-6$.

[145] Porod, G. Die röntgenkleinwinkelstreuung von dichtgepackten kolloiden systemen. Colloid \& Polymer Science 124, 2 (1951), 83-114.

[146] Lenormand, P., Lecomte, A., Dauger, A., Mary, C., And Guinebretiere, R. Reflectometry and small angle x-ray scattering studies of thin films prepared by sol-gel processing. vol. 10, pp. $255-64$.

[147] TeixeIrA, J. Small-angle scattering by fractal systems. Journal of Applied Crystallography 21, 6 (Dec 1988), 781-785. 
[148] Bale, H. D., And Schmidt, P. W. Small-angle x-ray-scattering investigation of submicroscopic porosity with fractal properties. Phys. Rev. Lett. 53 (Aug 1984), 596-599.

[149] Sinha, S. K., Sirota, E. B., Garoff, S., and Stanley, H. B. X-ray and neutron scattering from rough surfaces. Phys. Rev. B 38 (Aug 1988), 22972311 .

[150] Windover, D., Lu, T.-M., Lee, S. L., Kumar, A., Bakhru, H., Jin, C., AND LEE, W. Energy-dispersive, x-ray reflectivity density measurements of porous sio[sub 2] xerogels. Applied Physics Letters 76, 2 (2000), 158-160.

[151] Vacher, R., And Courtens, E. Phonon-fracton crossover and self-similarity in real fractals. Physica Scripta 1989, T29 (1989), 239.

[152] Courtens, E., Vacher, R., Pelous, J., And Woignier, T. Observation of fractons in silica aerogels. Europhys. Lett. (Switzerland) 6, 3 (1988/06/01), $245-50$.

[153] Vacher, R., Courtens, E., Coddens, G., Heidemann, A., Tsujimi, Y., Pelous, J., And Foret, M. Crossovers in the density of states of fractal silica aerogels. Phys. Rev. Lett. 65 (Aug 1990), 1008-1011.

[154] Calemczuk, R., De Goer, A. M., Salce, B., Maynard, R., And Zarembowitch, A. Low-temperature properties of silica aerogels. EPL (Europhysics Letters) 3, 11 (1987), 1205.

[155] de Goer, A. M., Calemczuk, R., Salce, B., Bon, J., Bonjour, E., And MAYNARD, R. Low-temperature energy excitations and thermal properties of silica aerogels. Phys. Rev. B 40 (Oct 1989), 8327-8335.

[156] Bernasconi, A., Sleator, T., Posselt, D., Kuems, J. K., And Ott, H. R. Dynamic properties of silica aerogels as deduced from specific-heat and thermal-conductivity measurements. Phys. Rev. B 45 (May 1992), 1036310376 .

[157] Wolf, A., And BRendel, R. Thermal conductivity of sintered porous silicon films. Thin Solid Films 513, 1-2 (2006), 385 - 390.

[158] Bruggeman, D. A. G. Berechnung verschiedener physikalischer konstanten von heterogenen substanzen. i. dielektrizitätskonstanten und leitfähigkeiten der mischkörper aus isotropen substanzen. Annalen der Physik 416, 7 (1935), 636664.

[159] Fricke, J., Lu, X., Wang, P., Buttner, D., And Heinemann, U. Optimization of monolithic silica aerogel insulants. International Journal of Heat and Mass Transfer 35, 9 (1992), 2305 - 2309. 
[160] Emmerling, A., AND Fricke, J. Scaling properties and structure of aerogels. Journal of Sol-Gel Science and Technology 8, 1-3 (1997), 781-788.

[161] Hopkins, P. E., Kaehr, B., Piekos, E. S., Dunphy, D., And Brinker, C. J. Minimum thermal conductivity considerations in aerogel thin films. Journal of Applied Physics 111, 11 (2012), 113532.

[162] Benedetti, A., Fagherazzi, G., Riello, P., Zeng, Y. W., Pinna, F., And Signoretto, M. Fractal properties of a partially crystalline zirconium oxide aerogel. Journal of Applied Crystallography 26, 5 (Oct 1993), 717-720.

[163] Shenoy, S., Barrera, E., and Bayazitoglu, Y. Non-fourier 3-omega method for thermal conductivity measurement. Nanoscale and Microscale Thermophysical Engineering 14, 3 (2010), 123 - 136.

[164] Kelly, B. The effect of defects on the basal plane thermal conductivity of a graphite crystal. Carbon 5, 3 (1967), $247-260$.

[165] Singh, D., Murthy, J. Y., And Fisher, T. S. Mechanism of thermal conductivity reduction in few-layer graphene. Journal of Applied Physics 110, 4 (2011), 044317.

[166] Balandin, A. A. Thermal properties of graphene and nanostructured carbon materials. Nat Mater 10, 8 (08 2011), 569-581.

[167] Gallego, N. C., and Klett, J. W. Carbon foams for thermal management. Carbon 41, 7 (2003), $1461-1466$.

[168] Donnet, J. Carbon Fibers, Third Edition,. Taylor \& Francis, 1998.

[169] Heremans, J., Rahim, I., and Dresselhaus, M. S. Thermal conductivity and raman spectra of carbon fibers. Phys. Rev. B 32 (Nov 1985), 6742-6747.

[170] Mayhew, E., and Prakash, V. Thermal conductivity of individual carbon nanofibers. Carbon 62, 0 (2013), 493 - 500.

[171] Qiu, L., Zheng, X., Zhu, J., Su, G., And Tang, D. The effect of grain size on the lattice thermal conductivity of an individual polyacrylonitrile-based carbon fiber. Carbon 51, 0 (2013), 265 - 273.

[172] Piraux, L., Nysten, B., Haquenne, A., Issi, J.-P., Dresselhaus, M., AND Endo, M. The temperature variation of the thermal conductivity of benzene-derived carbon fibers. Solid State Communications 50, 8 (1984), 697 700 .

[173] Fourdeux, A., Herinckx, C., Perret, R., and Ruland, W. The structure of carbon fibers (la structure des fibres de carbone). C. R. Acad. Sci. Paris (1970/07/),6p -. Translations;. 
[174] Gupta, A., Harrison, I. R., And LahiJani, J. Small-angle X-ray scattering in carbon fibers. Journal of Applied Crystallography 27, 4 (Aug 1994), 627-636.

[175] Peebles, L. Carbon fibers: formation, structure, and properties. CRC Press, 1995.

[176] Chawla, K. Fibrous Materials. Cambridge Solid State Science Series. Cambridge University Press, 2005.

[177] Chung, D. Materials for thermal conduction. Applied Thermal Engineering 21, 16 (2001), $1593-1605$.

[178] Atwater, M. A., Mousavi, A. K., Leseman, Z. C., And Phillips, J. Direct synthesis and characterization of a nonwoven structure comprised of carbon nanofibers. Carbon 5\%, 0 (2013), $363-370$.

[179] Atwater, M. A., Phillips, J., Doorn, S. K., Luhrs, C. C., Fernández, Y., Menéndez, J., And Leseman, Z. C. The production of carbon nanofibers and thin films on palladium catalysts from ethylene-oxygen mixtures. Carbon 47, 9 (2009), $2269-2280$.

[180] Tuinstra, F., And Koenig, J. L. Raman spectrum of graphite. The Journal of Chemical Physics 53, 3 (1970), 1126-1130.

[181] Ferrari, A. C., AND Robertson, J. Interpretation of raman spectra of disordered and amorphous carbon. Phys. Rev. B 61 (May 2000), 14095-14107.

[182] Matthews, M. J., Pimenta, M. A., Dresselhaus, G., Dresselhaus, M. S., AND Endo, M. Origin of dispersive effects of the raman d band in carbon materials. Phys. Rev. B 59 (Mar 1999), R6585-R6588.

[183] Shioya, M., And TAKaku, A. Characterization of microvoids in carbon fibers by absolute small-angle x-ray measurements on a fiber bundle. J. Appl. Phys. (USA) 58, $11(1985 / 12 / 01), 4074-82$.

[184] Sun, W.-C., Huang, M.-J., Chien, H.-C., Chang, T.-Y., And YaO, D.-J. A novel method for measuring thick film thermal conductivity. In Nano/Micro Engineered and Molecular Systems (NEMS), 2010 5th IEEE International Conference on (Jan 2010), pp. 1052-1056.

[185] Bhattacharyya, R. K. Heat-transfer model for fibrous insulations, thermal insulation performance. American Society for Teasting and Materials, 1980, pp. $272-286$.

[186] Research, G. E. C. C., And Development. Heat Transfer and Fluid Flow Data Books. Genium Publishing, 1984.

[187] FRICKE, H. A mathematical treatment of the electric conductivity and capacity of disperse systems i. the electric conductivity of a suspension of homogeneous spheroids. Phys. Rev. 24 (Nov 1924), 575-587. 
[188] StARK, C., AND FRICKE, J. Improved heat-transfer models for fibrous insulations. International Journal of Heat and Mass Transfer 36, 3 (1993), $617-625$.

[189] Evans, W. J., Shen, M., And Keblinski, P. Inter-tube thermal conductance in carbon nanotubes arrays and bundles: Effects of contact area and pressure. Applied Physics Letters 100, 26 (2012), 261908.

[190] Yang, J., Waltermire, S., Chen, Y., Zinn, A. A., Xu, T. T., And Li, D. Contact thermal resistance between individual multiwall carbon nanotubes. Applied Physics Letters 96, 2 (2010),-.

[191] Bauer, M. L., Pham, Q. N., Saltonstall, C. B., and Norris, P. M. Thermal conductivity of vertically aligned carbon nanotube arrays: Growth conditions and tube inhomogeneity. Applied Physics Letters 105, 15 (2014), -.

[192] Reich, S., Thomsen, C., And Maultzsch, J. Carbon Nanotubes: Basic Concepts and Physical Properties. Wiley-VCH, 2004.

[193] Jorio, A., Dresselhaus, M. S., And Dresselhaus, G. Carbon: Nanotubes Advanced topics in the Synthesis, Structures, Properties and Applications. Springer, 2001.

[194] Shaikh, S., Li, L., Lafdi, K., And Huie, J. Thermal conductivity of an aligned carbon nanotube array. Carbon (UK) 45, 13 (2007), 2608 - 13.

[195] XIE, H., CAi, A., And Wang, X. Thermal diffusivity and conductivity of multiwalled carbon nanotube arrays. Physics Letters A 369, 1-2 (9 2007), 120123.

[196] Gu, W., Lin, W., Yao, Y., And Wong, C. Synthesis of high quality, closely packed vertically aligned carbon nanotube array and a quantitative study of the influence of packing density on the collective thermal conductivity. In Electronic Components and Technology Conference (ECTC), 2011 IEEE 61st (05 2011), IEEE, pp. 1239-1243.

[197] Yu, C., Shi, L., Yao, Z., Li, D., And Majumdar, A. Thermal conductance and thermopower of an individual single-wall carbon nanotube. Nano Letters 5, 9 (2005), 1842-1846.

[198] Fujit, M., Zhang, X., Xie, H., Ago, H., Takahashi, K., Ikuta, T., Abe, H., And Shimizu, T. Measuring the thermal conductivity of a single carbon nanotube. Phys. Rev. Lett. 95 (Aug 2005), 065502.

[199] Pop, E., Mann, D., Wang, Q., Goodson, K., And Dai, H. Thermal conductance of an individual single-wall carbon nanotube above room temperature. Nano Letters 6, 1 (2006), 96-100. 
[200] Salaway, R. N., and Zhigilei, L. V. Molecular dynamics simulations of thermal conductivity of carbon nanotubes: Resolving the effects of computational parameters. International Journal of Heat and Mass Transfer 70, 0 (2014), $954-964$.

[201] Maruyama, S. A molecular dynamics simulation of heat conduction in finite length swnts. Physica B: Condensed Matter 323, 1-4 (2002), 193 - 195. Proceedings of the Tsukuba Symposium on Carbon Nanotube in Commemoration of the 10th Anniversary of its Discovery.

[202] Hashimoto, A., Suenaga, K., Gloter, A., Urita, K., and Iijima, S. Direct evidence for atomic defects in graphene layers. Nature 430, $7002(08$ 2004), 870-873.

[203] Kondo, N., Yamamoto, T., and Watanabe, K. Molecular-dynamics simulations of thermal transport in carbon nanotubes with structural defects. e-Journal of Surface Science and Nanotechnology 4 (2006), 239-243.

[204] Pettes, M. T., And Shi, L. Thermal and structural characterizations of individual single-, double-, and multi-walled carbon nanotubes. Advanced Functional Materials 19, 24 (2009), 3918-3925.

[205] Yang, B., Liu, J. L., Wang, K. L., and Chen, G. Simultaneous measurements of seebeck coefficient and thermal conductivity across superlattice. Applied Physics Letters 80, 10 (2002), 1758-1760.

[206] Aliev, A. E., Lima, M. H., Silverman, E. M., and Baughman, R. H. Thermal conductivity of multi-walled carbon nanotube sheets: radiation losses and quenching of phonon modes. Nanotechnology 21, 3 (2010), 035709.

[207] Hata, K., Futaba, D. N., Mizuno, K., Namai, T., Yumura, M., and IIJIMA, S. Water-assisted highly efficient synthesis of impurity-free singlewalled carbon nanotubes. Science 306, 5700 (2004), 1362-1364.

[208] Tong, T., Zhao, Y., Delzeit, L., Kashani, A., Meyyappan, M., and MajumDar, A. Dense vertically aligned multiwalled carbon nanotube arrays as thermal interface materials. Components and Packaging Technologies, IEEE Transactions on 30, 1 (march 2007), $92-100$.

[209] Zhang, Q., Huang, J.-Q., Zhao, M.-Q., Qian, W.-Z., and Wei, F. Modulating the diameter of carbon nanotubes in array form via floating catalyst chemical vapor deposition. Applied Physics A 94, 4 (2009), 853-860.

[210] Laurent, C., Flahaut, E., and Peigney, A. The weight and density of carbon nanotubes versus the number of walls and diameter. Carbon 48, 10 (2010), $2994-2996$. 